Programa de Doctorado en Ingeniería Matemática,

Estadística e Investigación Operativa

$$
\text { por la }
$$

Universidad Complutense de Madrid

$$
\text { y la }
$$

Universidad Politécnica de Madrid
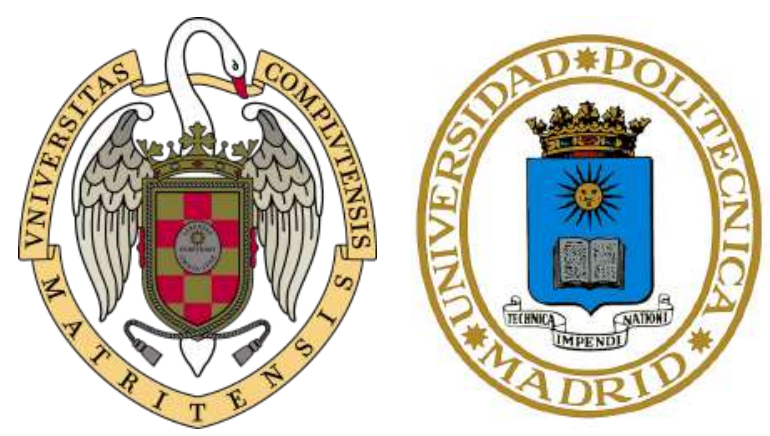

\title{
Análisis de estabilidad de flujos viscosos e incompresibles de densidad estratificada mediante métodos numéricos de alto y bajo orden
}

\author{
Tesis Doctoral \\ Ángel de Andrea González \\ Director \\ Leo Miguel González Gutiérrez \\ Año \\ 2020
}



Tribunal nombrado por el Mgfco. y Excemo. Sr. Rector de la Universidad Politécnica de Madrid, el día de de

Presidente

Vocal

Vocal

Vocal

Vocal Secretario D.

D.

D.

D.

D.
Realizado el acto de defensa y lectura de la Tesis el día de de , en

Calificación:

EL PRESIDENTE

LOS VOCALES 



\section{Abstract}

The present work deals with several types of fluid instabilities such as the Rayleigh-Taylor instability (RTI), Kelvin-Helmholtz instability (KHI), and Centrifugal instability (CTI) for incompressible, viscous and stratified density flows. Firstly, the computation of the spectrum of eigenvalues and eigenfunctions of linear problems derived from the RTI, KHI and CTI is performed by numerically solving the corresponding eigenvalue problem (EVP). Both the RTI and KHI are studied in one-dimensional (1D) and two-dimensional (2D) geometries, whereas the CTI is only analysed in 2D geometry. These canonic cases are extended to different versions where viscosity, surface tension and an stratified density distributions are added to the problem and the changes in the spectrum and the modal structure of the dominant modes is studied. The importance of extending the results to the two-dimensional case is twofold. First, it opens the possibility of generalizing the computation to more complex geometries that could contain fixed or floating bodies and second, allows the computation of flow instabilities of base flows which are particular solutions of the steady Navier-Stokes solutions. Secondly, for the RTI, it was found useful to study stability by using the initial value problem approach (IVP), as consequence we ensure the inclusion of certain continuum modes, otherwise neglected. This methodology includes a branch cut in the complex plane, consequently, in addition to discrete modes (surface RTI modes), a set of continuum modes (internal RTI modes) also appears. As a result, the usual information given by the normal mode analysis is now completed. Furthermore, a new role is found for surface tension, transforming surface RTI modes into internal RTI modes belonging to a continuous spectrum at a critical wavenumber. As a consequence, the cut-off wavenumber disappears: i.e. the growth rate of the RTI surface mode does not decay to zero at the cut-off wavenumber, as previous researchers used to believe. Finally, we found that the Rayleigh-Taylor instability exhibits essentially different time asymptotic behavior above and below the critical wavenumber.

This thesis collects results from two publications (JCR), as well some original work. 



\section{Resumen}

Este trabajo trata sobre sobre el estudio de varios tipos inestabilidades de fluidos como son la inestabilidad de Rayleigh-Taylor (RTI), la inestabilidad de Kelvin-Helmholtz (KHI) y la inestabilidad Centrífuga (CTI), para flujos viscosos, incompresibles y de densidad estratificada.

En primer lugar, se lleva a cabo el cálculo del espectro de autovalores y autofunciones del problema lineal asociado a la RTI, KHI y CTI resolviendo numéricamente el correspondiente problema de autovalores (EVP). La RTI y la KHI son estudiadas tanto en geometrías unidimensionales (1D) como bidimensionales (2D), mientras que la CTI es solo analizada en una geometría 2D. Estos problemas canónicos se extienden a diferentes versiones donde la viscosidad, tensión superficial y una distribución de densidad estratificada se añaden al problema, estudiándose los cambios en el espectro de autovalores y la estructura de los modos dominantes. La importancia de extender los resultados a un caso bidimensional es doble: se abre la posibilidad de generalizar el cálculo a geometrías más complejas que podrían contener cuerpos fijos o flotantes y permite el cálculo de inestabilidades de flujos base que son soluciones particulares de las ecuaciones de Navier-Stokes estacionarias.

En segundo lugar, se ha encontrado útil plantear el estudio de estabilidad de un fluido como un problema de valor inicial (IVP). Como consecuencia, podemos asegurar la inclusión de ciertos modos que pertenecen a un espectro continuo que, mediante otro enfoque, hubiesen pasado desapercibidos. El planteamiento IVP muestra la existencia de un corte de ramificación de la perturbación en el plano complejo que estaría asociado a la existencia del espectro continuo de modos RTI internos y que se suma a la del espectro discreto de modos RTI superficiales. De esta forma, podemos decir que los resultados obtenidos por un enfoque EVP se ven complementados por los hallados mediante un planteamiento tipo IVP. Además, se ha encontrado un nuevo rol para la tensión superficial: transformar, para un número de onda crítico, los modos RTI superficiales en modos internos RTI. Consecuentemente, el número de onda de corte desaparece: i.e. la tasa de crecimiento de la inestabilidad de los modos RTI superficiales no alcanza el valor de cero en el mencionado número de onda de corte, como se solía creer. Finalmente, se encuentra que la RTI muestra diferentes comportamientos temporales asintóticos por encima y por debajo del valor del número de onda crítico. 



\section{Artículos, ponencias y comunicaciones}

La calidad de esta tesis doctoral está avalada por la publicación de parte de su contenido en dos artículos de revistas de reconocido prestigio incluidas en el catálogo JCR, una comunicación en un congreso internacional y dos ponencias.

\section{Artículos}

- A. de Andrea González and L. M. González-Gutiérrez. Effects of a semi-infinite stratification on the Rayleigh-Taylor instability in an interface with surface tension. AIP Advances, 7(9): 095319, 2017.

- L. M. González-Gutiérrez and A. de Andrea González. Numerical computation of the Rayleigh-Taylor instability for a viscous fluid with regularized interface properties. Phys. Rev. E , 100:013101, 2019.

\section{Comunicaciones}

- A. de Andrea González. Effects of a semi-infinite stratification on Rayleigh-Taylor instability in an interface with surface tension. 17th International Conference on Atomic Processes in Plasmas. Queen's University. Belfast, 2011.

\section{Ponencias}

- A. de Andrea González. The continuous spectrum in Physics of Fluids. Primera Jornada PhDay Complutense-Ciencias Matemáticas. Escuela de Doctorado de la UCM. Madrid, 2018.1

\footnotetext{
${ }^{1}$ Por su calidad científica, esta ponencia fue galardonada con un accésit por un jurado de seis profesores adscritos al Programa de Doctorado de Ingeniería Matemática, Estadística e Investigación Operativa y al Programa de Doctorado de Investigación Matemática.
} 
- A. de Andrea González. Computación numérica de la inestabilidad de Rayleigh-Taylor para un fluido viscoso para propiedades regularizadas en la interfaz. Segunda Jornada PhDay Complutense-Ciencias Matemáticas. Escuela de Doctorado de la UCM. Madrid, 2019. 


\section{Agradecimientos}

Debo agradecer de manera especial y sincera al Dr. Leo Miguel González Gutiérrez, profesor titular de la Escuela Técnica Superior de Ingenieros Navales de la Universidad Politécnica de Madrid y miembro del Canal de Ensayos Hidrodinámicos (CEHINAV), el haber aceptado la dirección de esta tesis doctoral. Su apoyo, confianza en mi trabajo y su capacidad para guiar mis ideas ha sido un aporte invaluable, no solamente en el desarrollo de esta tesis, sino también en mi formación como investigador. Las ideas propias, siempre enmarcadas en su orientación y rigurosidad, han sido la clave del buen trabajo que hemos realizado juntos, el cual no se puede concebir sin su siempre oportuna participación. Le agradezco también su disponibilidad, paciencia, empatía y el haberme facilitado siempre los medios suficientes para llevar a cabo todas las actividades propuestas durante el desarrollo de esta tesis. Muchas gracias Leo y, como no podía ser de otra manera, espero seguir colaborando contigo en futuros trabajos de investigación.

De igual manera, mi más sincero agradecimiento a la Dra. Begoña Vitoriano Villanueva, profesora titular del Departamento de Estadística e Investigación Operativa de la Facultad de Matemáticas de la Universidad Complutense de Madrid, quien fue tuvo el gran acierto de ponerme en contacto con el que sería mi director de tesis.

Asimismo, agradezco al Dr. Antonio Souto Iglesias, profesor titular de la Escuela Técnica Superior de Ingenieros Navales de la UPM y miembro del CEHINAV, así como a los doctores Javier Calderón Sánchez, Héctor Rubén Díaz Ojeda y Amadeo Morán Guerrero la amabilidad mostrada hacia mi persona durante aquellas tardes de trabajo con mi director de tesis en el CEHINAV.

También me gustaría agradecer a la Dra. Silvia Noemí Santalla Arribas, a la Dra. Rosa María de la Cruz Fernández, y a la Dra. Paola Parente, profesora visitante, profesora titular, e investigadora, respectivamente, del Departamento de Física de la Universidad Carlos III de Madrid del que soy profesor asociado, el haberme brindado todo su apoyo y ánimo para finalizar esta ardua tarea, y sobre todo su cariño y amistad.

Y, por supuesto, el agradecimiento más profundo y sentido va para mi familia. Sin su apoyo, colaboración e inspiración habría sido imposible llevar a cabo esta dura empresa. 

A Florentino de Andrea Casas (mi abuelo) y a Daniel Miguel Herranz (mi bisabuelo), quienes desempeñando su profesión en la central hidroeléctrica de Serviliano convivieron con los vórtices y turbulencias del río Eresma a su paso por Bernardos (Segovia) 
Trabaja, termina y publica

- Michael Faraday 


\section{Índice general}

Abstract I

Resumen III

\begin{tabular}{|l|l|l|l|l}
\hline Artículos, ponencias y comunicaciones & V
\end{tabular}

\begin{tabular}{|ll}
\hline Agradecimientos & VII
\end{tabular}

1. Introducción 1

1.1. Teoría de estabilidad lineal . . . . . . . . . . . . . 1

1.2. El concepto de estabilidad global . . . . . . . . . . . . . 4

1.3. Casuística del análisis modal de estabilidad lineal . . . . . . . 5

1.4. Inestabilidad de flujos de densidad constante . . . . . . . . 6

1.4.1. Flujos de vórtices. . . . . . . . . . . . . . 6

1.4.2. Flujos con capa de cortadura entre corrientes paralelas 9

1.5. Inestabilidad de fluidos de densidad variable . . . . . . . . . . 11

1.5.1. Inestabilidad de Rayleigh-Taylor . . . . . . . . . . . . 11

$\begin{array}{lll}\text { 1.5.2. Inestabilidad Kelvin-Helmholtz en un medio heterogéneo } 20 & 0\end{array}$

1.5.3. La inestabilidad de vórtices con densidad variable . . 23

1.5.4. La inestabilidad de torbellino de densidad variable con jet axial. . . . . . . . . . . . . . 24

1.6. Organización de la tesis . . . . . . . . . . . 26

2. Metodología. Herramientas matemáticas 29

2.1. Las ecuaciones de Navier-Stokes . . . . . . . . . . . . . . . . . 29

2.2. Análisis modal de las LNSE . . . . . . . . . . . . . . . . . 31

2.2.1. Análisis BiGlobal de la inestabilidad: LNSE en dos dimensiones (2D) . . . . . . . . . . . . . 31

2.2.2. Análisis local de la inestabilidad: LNSE en una dimensión (1D) . . . . . . . . . . . . . . . . . 42

2.3. Análisis no modal de estabilidad . . . . . . . . . . . . 47

2.3.1. La técnica de la la transformada de Laplace . . . . . . 48 


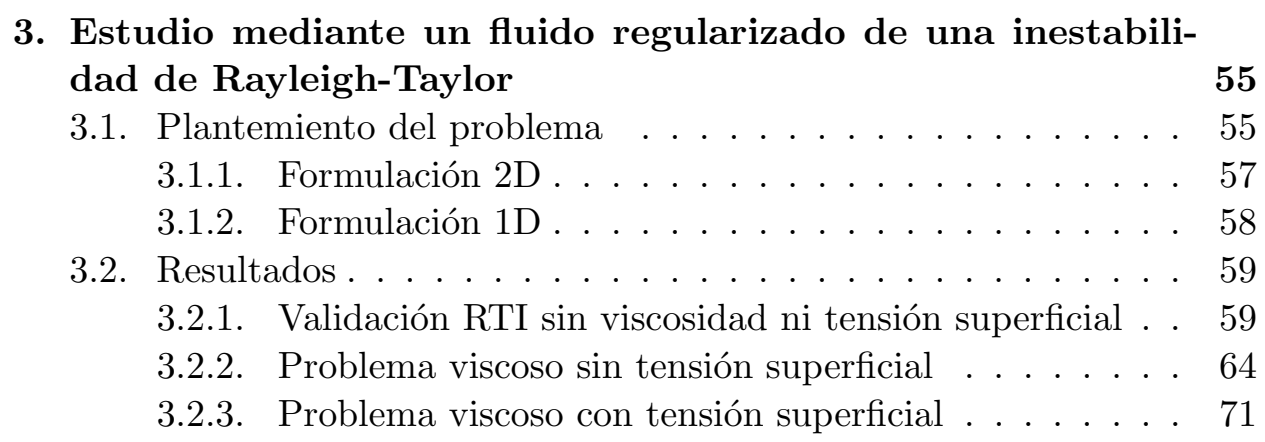

3.3. Conclusiones . . . . . . . . . . . . . . 76

4. Inestabilidad de Kelvin-Helmholtz (KHI) en un medio heterogéneo 81

4.1. Planteamiento del problema . . . . . . . . . . . . . . . . . 82

4.1.1. Formulación 1D . . . . . . . . . . . . . . . 86

4.1.2. Formulación 2D . . . . . . . . . . . . . . . 86

4.2. Resultados . . . . . . . . . . . . . . . . . . . . . . . . . . . . 88

4.2.1. Validación de la formulación 1D . . . . . . . . 88

4.2.2. Efecto del espesor de la capa de cortadura en la KHI. 90

4.2.3. Evolución conjunta de KHI y RTI . . . . . . . . . . . 99

4.3. Conclusiones . . . . . . . . . . . . . . . . . . . . . . . 108

5. Análisis BiGlobal de la inestabilidad de vórtices de densidad $\begin{array}{ll}\text { variable } & 111\end{array}$

5.1. Flujo base del problema . . . . . . . . . . . . . . . . . . 111

5.2. Resolución numérica de las LNSE mediante una malla 2D . . 113

5.2.1. Bqv de densidad constante. Validación de la formulación 2D . . . . . . . . . . . . . . . 113

5.2.2. Bqv de densidad variable . . . . . . . . . . 116

5.2.3. Vórtice de Lamb-Oseen de densidad variable . . . . . 122

5.3. Conclusiones . . . . . . . . . . . . . . . 123

6. El continuum en la RTI con tensión superficial $\quad 127$

6.1. Planeamiento del problema. Análisis lineal de estabilidad . . 127

$6.2 . \quad$ Relación de dispersión . . . . . . . . . . . . . . . . . . . 129

6.3. Evolución temporal de la perturbación . . . . . . . . . . . . . 134

6.3.1. Análisis asintótico para tiempos grandes $(\mathrm{t}>>1)$. . . 134

6.4. Resultados numéricos . . . . . . . . . . . . . . 138

6.4.1. Relación de dispersión . . . . . . . . . . . . . . . 138

6.4.2. Evolución temporal de la perturbación . . . . . . . . . 145 
Índice general

6.5. Resumen y conclusión . . . . . . . . . . . . . . . . . 151

7. Conclusiones 153

7.1. Resumen de los logros alcanzados . . . . . . . . . . . . . . 153

7.2. Trabajo futuro . . . . . . . . . . . . . . 155

\begin{tabular}{ll}
\hline Bibliografía & 157
\end{tabular} 



\section{Índice de figuras}

1.1. Inestabilidad de Rayleigh-Taylor. En nuestro caso $\rho_{1}>\rho_{2} ; \mathbf{g}$ es la intensidad de campo gravitatorio; se puede comprobar $\begin{array}{lll}\text { que el gradiente de densidad } \nabla \rho \text { y g tienen sentidos opuestos. } & 12\end{array}$

1.2. Inestabilidad de Rayleigh-Taylor en un sistema de dos fluidos en rotación de densidades $\rho_{1}$ y $\rho_{2}$ y con velocidad angular axial $\boldsymbol{\Omega}$. En nuestro caso $\rho_{1}>\rho_{2} ; \mathbf{g}_{\text {ef }}$ es la aceleración de la gravedad efectiva (aceleración centrífuga de inercia). Se puede comprobar que $\nabla \rho$ y g gef $_{\text {ef }}$ tienen sentidos opuestos. $\ldots \ldots . \quad \ldots 17$

1.3. Inestabilidad de Kelvin-Helmholtz en un sistema de dos flujos horizontales, paralelos, de diferentes densidades $\rho_{1}$ y $\rho_{2}$ y velocidades $\mathbf{U}_{\mathbf{1}}$ y $\mathbf{U}_{\mathbf{2}}$. En la interfase de separación entre los dos flujos se observa el inicio de la formación de vórtices. . . 20

2.1. El contorno $C$ de la tranformada inversa de Laplace para el caso general donde la transformada tiene singularidades $s_{1}$, $s_{2}$ y $s_{3}$ en el semiplano izquierdo y el semiplano derecho de $f(s)$. El contorno tiene parte real $s_{o} . \ldots \ldots \ldots$

2.2. El contorno $C$ de la figura 2.1, que ahora denominaremos $C_{o}$ se cierra a la izquierda con una arco de circunferencia $C_{R} . \quad 50$

3.1. Representación de la geometría utilizada para una simulación 2D. La línea discontinua representa la coordenada $y=0$. Las condiciones de contorno son establecidas también en cada frontera. . . . . . . . . . . . . . . 58

3.2. Tasa de crecimiento $\sigma$ de la inestabilidad RTI del autovalor más inestable, para el caso no viscoso, frente al número de onda $k$ para diferentes valores del número de Atwood $A_{\rho}=0.2,0.4,0.6,0.8$ para $H=1$ y $L_{s}=0.01$. La expresión analítica (3.8) es añadida mediante líneas negras para establecer una comparativa. . . . . . . . . . . . 60

3.3. Tasa de crecimiento $\sigma$ del modo más inestable $(m=1)$ y de los tres siguientes modos de la RTI $(m=2,3,4)$ en función del número de onda $k$ para $A_{\rho}=0.2, L_{s}=0.203$ y $H=1$. Los resultados de la expresión analítica aproximada $(3.9)$ han sido añadidos para establecer la oportuna comparación. . . . 61 
3.4. Autofunciones de la componente vertical de la velocidad $\hat{v}$ correspondientes a los cuatro modos más inestables $m=1$ (parte superior izquierda), $m=2$ (parte superior derecha), $m=3$ (parte inferior izquierda), $m=4$ (parte inferior derecha) para números de onda $k=4$ y $k=6$ y $A_{\rho}=0.2$. Otros parámetros son $L_{s}=0.1$ y $\left.H=1.\right] \ldots \ldots \ldots 62$

3.5. Autofunciones para la componente de la velocidad vertical $\hat{v}$ correspondientes a los cuatro modos más inestables $m=1$ (parte superior izquierda), $m=2$ (parte superior derecha), $m=3$ (parte inferior izquierda), $m=4$ (parte inferior derecha) para los números de onda $k=1$ y $k=4$ y $A_{\rho}=0.8$. Otros parámetros son $L_{s}=0.203$ y $H=6$. . . . . . . . . . 63

3.6. Dependencia de la tasa de crecimiento $\sigma$ con respecto al número de onda $k$ para $A_{\rho}=A_{\mu}=0.1$ (parte superior), $A_{\rho}=A_{\mu}=$ 0.5 (parte intermedia) y $A_{\rho}=A_{\mu}=0.9$ (parte inferior). Las curvas superior e inferior se corresponden con $H=4$ y $H=2$, respectivamente. Anteriores cálculos numéricos [1] y los obtenidos en el presente estudio mediante el método espectral $\left(L_{s}=0.01\right.$ y $\left.N=256\right)$ son representados. . . . . . . . . 65

3.7. Autofunciones de los modos más inestables para la velocidad vertical, densidad y presión $m=1$ (arriba), $m=2$ (abajo) para el dominio 1D en el caso viscoso, donde $k=\pi, A_{\rho}=$

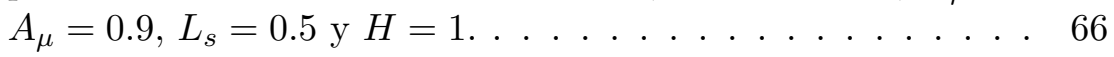

3.8. Dependencia de la tasa de crecimiento viscosa $\sigma$ con el número de onda $k$ siendo $A_{\rho}=A_{\mu}=0.9, L_{s}=0.5$ y $H=1$ para los cuatro modos más inestables $m=1,2,3,4$. . . . . . . . . . 67

3.9. Malla utilizada para el cálculo viscoso en 2D. . . . . . . . . . 68

3.10. Dependencia de la tasa de crecimiento viscosa $\sigma$ con respecto \begin{tabular}{|l|l|}
\hline al número de onda $k$ para $A_{\rho}=A_{\mu}=0.8,0.6,0.4,0.2$ y $H=$ \\
\hline 1. Los cálculos numéricos referenciados [1] y los obtenidos por \\
\hline FEM en 2D $\left(L_{s}=0.01\right)$ son representados. $\ldots \ldots \ldots .6 .69$
\end{tabular}

3.11. Estructura de la perturbación más inestable para el caso $k=$ $\pi, H=1, L=4$ y $A_{\mu}=A_{\rho}=0.9$. Desde la gráfica superior a la inferior: densidad, presión, velocidad horizontal y velocidad vertical. Los cálculos fueron llevados a cabo con el parámetro de interfaz $L_{s}=0.5$. . . . . . . . . . . . . . 70 
3.12. Estructura del segundo modo más inestable para el caso de $k=\pi, H=1$ y $A_{\mu}=A_{\rho}=0.9$. Desde la gráfica superior a la inferior: autofunciones de densidad, presión, velocidad horizontal y velocidad vertical. El cálculo se ha realizado para el parámetro de interfase $L_{s}=0.5$. . . . . . . . . . 72

3.13. Estructura del tercer modo más inestable para el caso de $k=$ $\pi, H=1$ y $A_{\mu}=A_{\rho}=0.9$. Desde la gráfica superior a la inferior: autofunciones de la densidad, presión, velocidad horizontal y velocidad vertical. El cálculo se ha realizado para el parámetro de interfase $L_{s}=0.5$. . . . . . . . . . . 73

3.14. Tasa de crecimiento de la inestabilidad en función del número de onda para $S=0,1,0.25$ siendo $A_{\mu}=A_{\rho}=1.0, L_{s}=0.01$

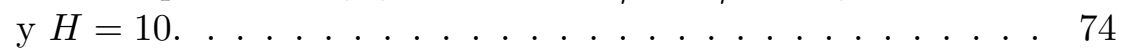

3.15. Tasa de crecimiento de la inestabilidad en función del número de onda para $S=1$ y $S=1 / 9$ siendo $A_{\mu}=A_{\rho}=1.0$, $L_{s}=0.01$ y $H=10 . \ldots \ldots \ldots \ldots 75$

3.16. Tasa de crecimiento de la inestabilidad en función del número de onda, para $A_{\rho}=A_{\mu}=0.2, L_{s}=0.01, H=1, S=0 \mathrm{y}$ $S=0.003 . \ldots \ldots \ldots \ldots \ldots \ldots$

3.17. Tasa de crecimiento de la inestabilidad en función del número de onda, para $A_{\rho}=A_{\mu}=0.6, L_{s}=0.01, H=1, S=0$ y $S=0.01 . \ldots \ldots \ldots \ldots \ldots \ldots 77$

3.18. Perturbación para el caso $k=\pi, H=1, L=4$ y $A_{\mu}=A_{\rho}=$ 0.2 y $S=0.003$. Desde la gráfica superior a la inferior se presentan las amplitudes de las perturbaciones de la densidad, presión, velocidad horizontal y velocidad vertical. Los cálculos fueron llevados a cabo con el parámetro de interfase $L_{s}=0.01$. 78

4.1. Representación del flujo base en velocidad y en densidad dado por la expresión (4.3) para $H=1, A_{\rho}=0.6$ y $U_{o}=1$. En la gráfica izquierda el flujo base está caracterizado por $L_{\rho}=$ $L_{u}=0.1$ (transición difusa) y en la derecha, $L_{\rho}=L_{\rho}=10^{-3}$ (transición abrupta). ................. 84

4.2. Comparativa entre flujos base en densidad (izquierda) y velocidad (derecha) para $U_{o}=1, H=1, A_{\rho}=0.6$ y $L_{\rho}=L_{u}=$ 0.1 . En línea discontinua se ha representado el flujo base dado por (4.5) y (4.6). En línea continua, el flujo base definido por

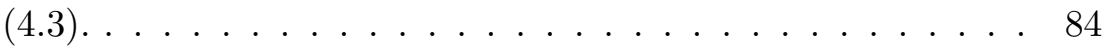


4.3. Distribución de vorticidad (gráfica superior) y gradiente de vorticidad (gráfica inferior) del flujo base dado por (4.3) para $U_{o}=1, H=1, L_{u}=0.1$. En línea discontinua se ha representado la distribución de vorticidad correspondiente al flujo base dado por (4.6). En línea continua, la vorticidad del flujo

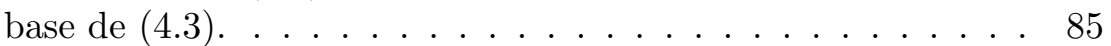

4.4. Representación de la geometría utilizada para un análisis 2D. La línea discontinua representa la coordenada $y=0$. Las condiciones de contorno para las perturbaciones son establecidas también en cada frontera. . . . . . . . . . . . . . . 87

4.5. Tasa de crecimiento de la inestabilidad $\sigma$ (gráfica superior) y frecuencia $\omega$ (gráfica inferior) frente al número de onda $k$ para diferentes valores del número de Atwood $A_{\rho}=0.2,0.4,0.6,0.8$ para $H=1, L_{\rho}=L_{u}=10^{-3}$ y el flujo base dado por (4.3). Mediante líneas se ha representado la expresión analítica (4.12) para establecer una comparativa. . . . . . . . . . . 89

4.6. Tasa de crecimiento normalizada $\sigma$ de la KHI pura frente al número de onda normalizado $k$ para $A_{\rho}=0.2, L_{\rho}=10^{-3}$,

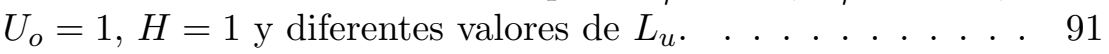

4.7. Malla utilizada para el análisis en 2D. . . . . . . . . . . . . 92

4.8. Tasa de crecimiento de la KHI $\sigma$ (gráfica superior) y frecuencia $\omega$ (gráfica inferior) frente al número de onda normalizado $k$ para $A_{\rho}=0.2, L_{\rho}=L_{u}=10^{-2}, H=1, U_{o}=1$. . . . . . . 93

4.9. Modo más inestable de la KHI para el caso $k=5 \pi / 4, H=1$, $L=4$ y $A_{\rho}=0.2$. Desde la gráfica superior a la inferior, amplitud de la perturbación de densidad, presión, velocidad horizontal y velocidad vertical. Los cálculos se han realizado con los parámetros de interfase $L_{s}=L_{u}=0.01 . \quad$. . . . . . . 94

4.10. Líneas de corriente de la perturbación para $k=5 \pi / 2, A_{\rho}=$ $0.2, H=1, U_{o}=1, L_{\rho}=0.01, L_{u}=0.01 . \ldots \ldots . . . .966$

4.11. Tasa de crecimiento $\sigma$ de la KHI (gráfica superior) y frecuencia $\omega$ (gráfica inferior) frente al número de onda normalizado $k$ para $A_{\rho}=0.2, L_{\rho}=0.01, H=1, L_{u}=0.1, U_{o}=1$. . . . . 97

4.12. Perturbación más inestable de la KHI pura el caso $k=5 \pi / 4$, $H=1, L=4$ y $A_{\rho}=0.2$. Desde la gráfica superior a la inferior, autofunciones de densidad, presión, velocidad horizontal y velocidad vertical. Los cálculos fueron llevados a cabo con los parámetros de interfase $L_{\rho}=0.01$ y $L_{u}=0.1$. . . . . . . 98

4.13. Líneas de corriente para $k=5 \pi / 2, A_{\rho}=0.2, H=1, U_{o}=1$, $L_{\rho}=0.01, L_{u}=0.1 . \ldots \ldots \ldots \ldots$. . . . . . . 99 
4.14. Modo más inestable de la KHI para el caso $k=\pi / 2, H=1$, $L=4$ y $A_{\rho}=0.2$. Desde la gráfica superior a la inferior, se muestran las amplitudes de las perturbaciones en densidad, presión, velocidad horizontal y velocidad vertical. Los cálculos fueron llevados a cabo con los parámetros de interfase $L_{\rho}=$ 0.01 y $L_{u}=0.1$. . . . . . . . . . . . . . . . . . . . . . . . 100

4.15. Líneas de corriente para $k=\pi / 2, A_{\rho}=0.2, H=1, U_{o}=1$, $L_{\rho}=0.01, L_{u}=0.1$. Se aprecian dos vórtices con vorticidad positiva (línea de color azul) y dos con vorticidad negativa (línea de color rojo). . . . . . . . . . . . . . . 101

4.16. Tasa de crecimiento de la KHI $\sigma$ (gráfica superior) y frecuencia $\omega$ (gráfica inferior) frente al número de onda normalizado $k$ para $A_{\rho}=0.2, L_{\rho}=0.3, H=1, L_{u}=0.01, U_{o}=1$. . . . . 102

4.17. Modo más inestable de la KHI para el caso $k=6 \pi / 4, H=1$, $L=4$ y $A_{\rho}=0.2$. Desde la gráfica superior a la inferior, se muestran las amplitudes de las perturbaciones en densidad, presión, velocidad horizontal y velocidad vertical. Los cálculos fueron llevados a cabo con los parámetros de interfase $L_{\rho}=$ 0.3 y $L_{u}=0.01 . \ldots \ldots \ldots$. . . . . . . . . . . . 103

4.18. Tasa de crecimiento normalizada de la inestabilidad $\sigma$ (RTI+KHI) frente al número de onda normalizado $k$ para $A_{\rho}=0.6$, $H=1, L_{\rho}=L_{u}=0.1,0.2,0.3,0.5, U_{o}=1 . \ldots . . . .104$

4.19. Tasa de crecimiento normalizada de la inestabilidad $\sigma$ frente al número de onda normalizado $k$ para $L_{\rho}=L_{u}=0.1,0.2,0.3,0.5$, $A_{\rho}=0.6, H=1, U_{o}=1$. Se representan por separado los casos individuales de RTI y KHI, así como el efecto combinado de las dos inestabilidades. . . . . . . . . . . . . 105

4.20. Tasa de crecimiento de la inestabilidad (RTI+KHI) $\sigma$ (gráfica superior) y frecuencia $\omega$ (gráfica inferior) frente al número de onda normalizado $k$ para $A_{\rho}=0.6, L_{\rho}=L_{u}=0.2, H=1$, $U_{o}=1 . \ldots \ldots \ldots \ldots 6$

4.21. Perturbación más inestable para el caso $k=\pi, H=1, L=4$ y $A_{\rho}=0.6$ (RTI+KHI). Desde la gráfica superior a la inferior, autofunción de densidad, presión, velocidad horizontal y velocidad vertical. Los cálculos fueron llevados a cabo con los parámetros de interfase $L_{\rho}=L_{u}=0.2$. . . . . . . . . . . 107

4.22. Líneas de corriente siendo $L_{\rho}=L_{u}=0.2, H=1, A_{\rho}=0.6$, $U_{o}=1$ y $k_{x}=\pi$, coexistiendo RTI y KHI. . . . . . . . . 108 
5.1. Malla para el análisis BiGlobal de la inestabilidad del Bqv aislado (imagen superior). También se muestra en detalle la parte central ampliada de la malla para su claridad (imagen inferior). . . . . . . . . . . . . . . . 114

5.2. Tasa de crecimiento de la inestabilidad $\sigma$ frente al número de onda axial $k_{z}$ para $R e=667, q=0.8$ y Bqv de densidad constante. . . . . . . . . . . . . . . . 115

5.3. Frecuencia $\omega$ frente al número de onda axial $k_{z}$ para $R e=667$, $q=0.8$ y Bqv de densidad constante. . . . . . . . . . 116

5.4. Tasa de crecimiento de la inestabilidad $\sigma$ (grafica superior) y frecuencia $\omega$ (gráfica inferior) frente al número de onda axial $k_{z}$ para un Bqv de densidad constante siendo $q=0.8$ y $R e \rightarrow \infty$.117

5.5. Tasa de crecimiento de la inestabilidad $\sigma$ (grafica superior) y frecuencia $\omega$ (gráfica inferior) frente al número de onda axial $k_{z}$ para un Bqv de densidad variable $\operatorname{con} s=2, R e=667$, $q=0.8$ y $\delta=\delta_{\rho}=1 . \ldots \ldots \ldots \ldots \ldots 119$

5.6. Comparativa de $\sigma$ frente a $k_{z}$ para $m=3$ y $m=4$ siendo $R e=667, q=0.8, s=2$ y $\delta=\delta_{\rho}=1$. Se han representado los resultados obtenidos en este trabajo junto con los de Di Pierro et al. [2]. . . . . . . . . . . . . . . . . . 120

5.7. Amplitud de la densidad perturbada para $R e=667, q=0.8$, $s=2, \delta=\delta_{\rho}=1$. En la gráfica izquierda se muestra la autofunción de la densidad perturbada para $m=3$ y $k_{z}=$ 2.25. En la gráfica derecha, para $m=4$ y $k_{z}=2.0$. . . . . . . 121

5.8. Amplitud de la densidad perturbada para $R e=667, q=0.8$, $s=2, \delta=\delta_{\rho}=1$. En la gráfica izquierda se muestra la autofunción de la densidad para $m=5$ y $k_{z}=3$. En la gráfica derecha, para $m=7$ y $k_{z}=4.6$. . . . . . . . . . 121

5.9. Tasa de crecimiento de la inestabilidad $\sigma$ (grafica superior) y frecuencia $\omega$ (gráfica inferior) frente al número de onda azimutal $m$ para $R e \rightarrow \infty, q=0.5, \epsilon=6.31, \delta=1$ y $s=3]$.

5.10. Amplitud de la densidad perturbada para $R e \rightarrow \infty, q=0.5$, $s=3, \epsilon=6.31, \delta=1$ y $k_{z}=0$. En la gráfica izquierda se muestra la autofunción de la densidad perturbada para $m=2$. En la gráfica derecha, para $m=3$. . . . . . . . . 125

5.11. Amplitud de la densidad perturbada para $R e \rightarrow \infty, q=0.5$, $s=3, \epsilon=6.31, \delta=1$ y $k_{z}=0$. En la gráfica izquierda se muestra la autofunción de la densidad perturbada para $m=4$. En la gráfica derecha, para $m=5 . \ldots \ldots 125$ 
6.1. Perfil de densidad en el estado no perturbado. . . . . . . . . . 128

6.2. a) El camino de integración de Bromwich $C$ con $\operatorname{Re}(s)=s_{o}$. Se aprecian en el eje real los polos $\pm s_{p}$ y los puntos de ramificación $\pm s_{b}$, representando las líneas onduladas el corte de ramificación correspondiente a la hoja de Riemann apropiada. b) Nuevo contorno para la evaluación de la integral (6.20)] . . 133

6.3. Contorno de integración $C^{\prime}$ utilizado para invertir $v_{k s}(y, s)$.

6.4. Dependencia del cuadrado de la tasa de crecimiento adimensional de la inestabilidad con el número de onda. Los resultados se han obtenido para $\tau=0.01$ y $\tau=0.02$ y diferentes números de Atwood $A_{\rho}=0.4$ (gráfica superior) y $A_{\rho}=0.7$ (gráfica inferior). Las líneas continuas corresponden al espectro discreto. Las líneas discontinuas representan los puntos de ramificación. Por simplicidad, se muestran los números de onda críticos $k_{c r}^{-}$y $k_{c r}^{+}$para $\tau=0.02 . \quad \ldots . . . . . . .141$

6.5. Número de onda crítico adimensional $k_{c r}$ en función del número de Atwood $A_{\rho}$ para diferentes valores del parámetro $\tau$. La rama inferior y la superior corresponden a $k_{c r}^{-}$y $k_{c r}^{+}$respectivamente. . . . . . . . . . . . . . . . . 142

6.6. Dependencia del cuadrado de la tasa de crecimiento adimensional frente al número de onda adimensional para $\tau=0.01$ y diferentes valores del número de Atwood $A_{\rho}=0.22$ (gráfica superior) y $A_{\rho}=0.2089422$ (gráfica inferior). Las líneas continuas se corresponden con nuestros cálculos numéricos (modos discretos). Las líneas discontinuas representan los puntos de ramificación. ..................... 143

6.7. Mapa de contorno de la parte imaginaria normalizada de $v_{k} s\left(y, y_{1} ; s, k\right)$ frente a $s$ para $A_{\rho}=0.7, k=6, y_{1}=0.5$, $y=0, \tau=0.01$ y $\Gamma_{o}=1$. Se muestra en una franja blanca el corte de ramificación en el eje real entre $R e(s)=-1 \mathrm{y}$ $R e(s)=1$. Cada pareja de lóbulos representa un polo. . . . . 144

6.8. Mapa de contorno de la parte imaginaria de $v_{k s}\left(y, y_{1} ; s, k\right)$ frente a $s$ para $A_{\rho}=0.7, k=20, y_{1}=0.5, y=0, \tau=0.01 \mathrm{y}$ $\Gamma_{o}=1 . \ldots \ldots \ldots \ldots \ldots$ 
xiv Índice de figuras

6.9. Representación de la función normalizada $v_{k}$ como función del tiempo para $A_{\rho}=0.7, \tau=0.01, k=6, \Gamma_{o}=1, y=7 \mathrm{y}$ $y_{1}=10^{-4}$. La línea sólida representa una función de ajuste de la forma $A e^{s_{p} t}$. La velocidad perturbada la representamos $v_{k}$ sobre una escala logarítmica. En esta gráfica están representadas simultáneamente las contribuciones del espectro continuo y del discreto. . . . . . . . . . . . . . . . . 147

6.10. Cociente entre la velocidad perturbada total en función de $y$ y la correspondiente al modo discreto para $A_{\rho}=0.7, \tau=0.01$, $k=6, \Gamma_{o}=1, y_{1}=10^{-4}$ y $y=10^{-3}, 1,5,10$. La línea discontinua es la asíntota donde $v_{k} \sim v_{k}^{*}$. . . . . . . . . 147

6.11. Dependencia de $\delta$ en $y$ para $\tau=10^{-3}, \Gamma_{o}=1, k=12$, $y_{1}=10^{-4}$ y diferentes números de Atwood $A_{\rho}=0.3,0.5,0.7$ y 0.9. La línea discontinua es la asíntota para la cual $\delta \sim 1]$.

6.12. Dependencia de $\delta$ con $k$ para $\tau=10^{-3}, y_{1}=10^{-4}, A_{\rho}=0.7$, $\Gamma_{o}=1$ y $y=1,5,10$, y $15 . \ldots \ldots \ldots 151$ 


\section{Índice de tablas}

1.1. Diferentes enfoques de análisis modal en la moderna teoría de estabilidad lineal: local (OSE), no local (PSE) y Global (BiGobal,TriGlobal y PSE-3D). . . . . . . . . . . 6

1.2. Comparativa entre modelos de vórtices . . . . . . . . . . 25

4.1. Estudio de convergencia para diferentes números de onda $k$. 95

5.1. Validación de los resultados obtenidos con los de Fabre et al. 3. . . . . . . . . . . . . . . . . . 113

5.2. Validación de los resultados obtenidos con los de Delbende et al. [4] para $R e=667, \delta=1$ y $q=0.8$. . . . . . . . . . . 115

5.3. Análisis de convergencia para $\delta=\delta_{\rho}=1(\epsilon=1), R e=667 \mathrm{y}$ $q=0.8$. . ...................... 118

5.4. Análisis convergencia para $R e \rightarrow \infty, q=0.5, \epsilon=6.31, \delta=1$ $\mathrm{y} s=3 \ldots \ldots \ldots \ldots \ldots \ldots \ldots \ldots$

6.1. Parámetros de ajuste $a, b s_{b}$ en la expresión $v_{k}\left(y=10^{-3}, t\right)=$ $a t^{-b} e^{\left(s_{b} t\right)}$ para diferentes valores de $k$, siendo $\Gamma_{o}=1, A_{\rho}=$ $0.7, \tau=0.01, y=10^{-3}$ y $y_{1}=10^{-4} \ldots \ldots \ldots 149$ 



\section{Capítulo 1 Introducción}

La estabilidad hidrodinámica se ocupa de la respuesta de un flujo laminar a una perturbación de pequeña amplitud. Si una vez perturbado, el flujo retorna a su régimen laminar se dice que el flujo es estable. Por el contrario, si la perturbación crece y da lugar a que el flujo laminar cambie a un diferente estado, se dice que el flujo es inestable. La teoría de la estabilidad está relacionada con el análisis matemático de la evolución de perturbaciones superpuestas a un flujo base laminar. En la mayoría de los casos se asume que las perturbaciones sean pequeñas de tal manera que simplificaciones posteriores puedan ser justificadas. En particular, siempre es deseable estudiar el problema de estabilidad mediante una ecuación lineal que gobierne la evolución de las perturbaciones.

En esta tesis doctoral nos centraremos exclusivamente en la teoría de estabilidad lineal que presupone que todas las perturbaciones son infinitesimales. Es importante resaltar que, desde el punto de vista físico, la estabilidad lineal debe ser considerada como un indicador de la evolución del sistema frente a perturbaciones. Un análisis lineal de la estabilidad es válido hasta que los efectos no lineales pasen a ser importantes.

En este capítulo, en primer lugar, describiremos el estado del arte en lo que concierne a la estabilidad de flujos. La literatura que se tratará a continuación se corresponde con aquellos fenómenos físicos en los que se sustenta la presente tesis doctoral. En segundo lugar, se hará una descripción de la organización del presente trabajo de investigación.

\subsection{Teoría de estabilidad lineal}

Los primeros estudios sobre la estabilidad lineal fueron llevados a cabo por Rayleigh (1880) [5] y Reynolds (1883) [6] quienes introdujeron las bases de las etapas iniciales en el proceso de transición de flujo laminar a turbulento. Por ello, podemos decir que una importante propiedad de un fluido en equilibrio es la estabilidad [7].

Para definir matemáticamente el término estabilidad necesitamos especificar alguna métrica para definir el significado del término pequeño. Por 
ejemplo, siguiendo a Drazin [8] utilizaremos la siguiente definición en el sentido de Liapounov. Sean $\tilde{\mathbf{u}}(\mathbf{x}, 0), \tilde{\rho}(\mathbf{x}, 0), \tilde{p}(\mathbf{x}, 0)$ las perturbaciones de la velocidad, densidad y presión de un flujo base en el instante de tiempo $t=0$, respectivamente, siendo $\mathbf{x}=(x, y, z)$ el vector de las coordenadas espaciales. Se dice que dicho flujo base es estable, si para todo $\epsilon>0$ existe $\delta(\epsilon)$ de tal forma que

$$
\|\tilde{\mathbf{u}}(\mathbf{x}, 0)\|,\|\tilde{\rho}(\mathbf{x}, 0)\|,\|\tilde{p}(\mathbf{x}, 0)\|<\delta
$$

entonces

$$
\|\tilde{\mathbf{u}}(\mathbf{x}, t)\|<\epsilon,\|\tilde{\rho}(\mathbf{x}, t)\|<\epsilon,\|\tilde{p}(\mathbf{x}, t)\|<\epsilon, \forall t>0
$$

siendo $\tilde{\mathbf{u}}(\mathbf{x}, t), \tilde{\rho}(\mathbf{x}, t), \tilde{p}(\mathbf{x}, t)$ las perturbaciones de la velocidad, densidad y presión del flujo en un instante de tiempo arbitrario $t$. De no ser así, se dice que el flujo base es inestable.

El flujo base se dice asintóticamente estable si es estable y, además,

$$
\|\tilde{\mathbf{u}}(\mathbf{x}, 0)\|,\|\tilde{\rho}(\mathbf{x}, 0)\|,\|\tilde{p}(\mathbf{x}, 0)\| \rightarrow 0, \text { si } t \rightarrow \infty
$$

En el marco de la mecánica de fluidos, la estabilidad de un flujo se puede estudiar mediante las perturbaciones de pequeña amplitud superpuestas a un flujo base del cual queremos estudiar su estabilidad. Si el flujo base es una solución de las ecuaciones de Navier-Stokes (NSE), el estudio de las perturbaciones queda descrito por las ecuaciones de Navier-Stokes linealizadas (LNSE). En general, no es necesario invocar un supuesto de flujo paralelo o débilmente paralelo y, por lo tanto, el flujo analizado con respecto a su estabilidad puede ser cualquier solución 2D o 3D de las ecuaciones de Navier-Stokes. Los flujos base laminares pueden ser estacionarios o no estacionarios. Dichos flujos base pueden ser analíticos en algunos casos u obtenerse mediante simulaciones numéricas directas 2D o 3D. En general, los flujos laminares estacionarios solo existen a números de Reynolds bajos, pero también existen procedimientos numéricos para la obtención de flujos base en condiciones en las que se esperaría una inestabilidad global lineal, basados en la continuación (Keller) [9] y la amortiguación de frecuencia selectiva (Askervik et al.) [10].

Desde el punto de vista matemático, el análisis de estabilidad lineal de las ecuaciones de Navier-Stokes está relacionado con la descomposición de cualquier magnitud del flujo $\mathbf{q}(\mathbf{x}, t)$ (velocidad, presión...) en un estado estacionario del flujo base $\mathbf{Q}(\mathbf{x})$ más la componente de la perturbación $\tilde{\mathbf{q}}(\mathbf{x}, t)$. Esta descomposición, en su forma más general, la podemos expresar como

$$
\mathbf{q}(\mathbf{x}, t)=\mathbf{Q}(\mathbf{x})+\epsilon \tilde{\mathbf{q}}(\mathbf{x}, t), \quad \epsilon<<1
$$


siendo $\mathbf{Q}(\mathbf{u}, P, R)^{T}=(U, V, W, P, R)^{T}, \tilde{\mathbf{q}}=(\tilde{\mathbf{u}}, \tilde{p}, \tilde{\rho})^{T}=(\tilde{u}, \tilde{v}, \tilde{w}, \tilde{p}, \tilde{\rho})^{T}$ donde el estado del flujo es definido por las componentes de la velocidad $\mathbf{u}$ en cada dirección, $U, V, W$, con respecto a los ejes $x, y, z$, respectivamente, la presión $P$ y la densidad $R$.

Sustituyendo la descomposición de cualquier magnitud del flujo dada por (1.4) en la ecuaciones de Navier-Stokes, sustrayendo las ecuaciones verificadas por el flujo base y despreciando los términos de las perturbaciones de orden $\mathcal{O}\left(\epsilon^{2}\right)$ debido a su pequeña amplitud, obtenemos el siguiente problema de valor inicial (IVP) para perturbaciones de pequeña amplitud

$$
\mathcal{B}(\mathbf{Q}, R e, F r) \frac{\partial \tilde{\mathbf{q}}}{\partial t}=\mathcal{A}(\mathbf{Q}, R e, F r) \tilde{\mathbf{q}}
$$

siendo $\mathcal{A}$ y $\mathcal{B}$ dos operadores lineales, Re el número de Reynolds y $F r$ el número de Froude. De esta forma, obtendremos las ecuaciones de NavierStokes linealizadas (LNSE).

En el caso de que el flujo base sea estacionario, la separación entre la coordenada temporal y las espaciales en (1.5) permite introducir una descomposición de Fourier en el tiempo a través de la expresión

$$
\tilde{\mathbf{q}}(\mathbf{x}, t)=\hat{\mathbf{q}}(\mathbf{x}) \exp (\gamma t)
$$

Por tanto, la solución general es una superposición lineal de modos normales.

Sustituyendo (1.6) en la ecuación (1.5) obtenemos el siguiente problema tridimensional de autovalores generalizado (EVP)

$$
\mathcal{A} \hat{\mathbf{q}}=\gamma \mathcal{B} \hat{\mathbf{q}}
$$

La discretización numérica de las direcciones espaciales y la imposición de condiciones de contorno apropiadas completan la formulación matricial. Utilizando este método, la estabilidad del flujo base vendrá determinada por los autovalores de (1.7). El autovalor $\gamma=\sigma+i \omega$ es un número complejo cuya parte real representa la tasa de crecimiento de la inestabilidad y la parte imaginaria la frecuencia de la perturbación. Así, el flujo se considera estable cuando la tasa de crecimiento de la inestabilidad $\sigma$ es negativa para cualquier perturbación. En este último caso, la aproximación lineal deja de ser válida cuando la amplitud de la perturbación es lo suficientemente grande para despreciar los términos no lineales.

En este contexto, nos referiremos a análisis modal o no modal, dependiendo si se resuelve un EVP o un IVP, respectivamente. 


\subsection{El concepto de estabilidad global}

Existe una terminología comúnmente aceptada para los casos particulares de flujos paralelos o asimétricos que dependen de una sola coordenada espacial (formulación 1D), en los cuales $\mathbf{Q}=\mathbf{Q}(y)$ (caso cartesiano) o $\mathbf{Q}=\mathbf{Q}(r)$ (polares) donde $y$ o $r$ denotan la coordenada normal a la pared que limita al fluido o la coordenada radial, respectivamente. En este contexto de flujos unidimensionales, nos referiremos a análisis modal local o no modal dependiendo de si se resuelve un EPV o IPV, respectivamente. El trabajo de Schmid y Henningson [11] muestra un compendio actualizado de la inestabilidad de estos flujos 1D tanto desde el punto de vista de un EPV como del IVP.

En el trabajo realizado en los primeros años 80 (Pierrehumbert y Widnall, 12], Eriksson y Rizzi [13], Pierrehumbert [14]) comenzó una nueva era basada en la solución de problemas de autovalores EVP que tenían como flujo base casos bidimensionales $\mathbf{Q}=\mathbf{Q}(x, y)$. En las últimas décadas, soluciones a este tipo de flujos base mediante problemas de valor inicial (IVP) han aparecido con frecuencia en la literatura, mientras que soluciones a problemas de autovalores con flujos base $3 \mathrm{D} \mathbf{Q}=\mathbf{Q}(x, y, z)$ han sido encontradas por primera vez y en el año 2006 por Tezuka y Suzuki [15]. La literatura actual se refiere a los análisis realizados tanto con EVP o IVP de flujos $\mathbf{Q}$ dependientes de dos o tres coordenadas espaciales como analisis de estabilidad global, donde los modos globales son los autovectores $\widehat{\mathbf{q}}$ del problema (1.7).

Para evitar posibles confusiones, los términos análisis de inestabilidad BiGlobal y TriGlobal han sido propuestos por Theofilis [16] para describir análisis (modales o no modales) de flujos base base dependientes de dos o tres coordenadas espaciales, respectivamente. Aunque esta terminología ha sido ampliamente utilizada en la literatura (Karniadakis y Sherwin [17], Longueteau y Brazier [18, Piot et al. [19]), términos alternativos como análisis de inestabilidad directa, empleado por Barkley et al. [20], o análisis armónico de las ecuaciones de Navier-Stokes linealizadas, usado por Carpenter et al. 21] son otras denominaciones de este tipo de análisis. La necesidad de una terminología que aclare el significado del análisis de inestabilidad global reconoce el riesgo de confusión con toda la terminología global aceptada; esta tesis utiliza la terminología BiGlobal y TriGlobal. 


\subsection{Casuística del análisis modal de estabilidad lineal}

Como podemos observar en la expresión (1.6), de forma general las perturbaciones $\tilde{\mathbf{q}}$ pueden ser expresadas como el producto de una función de fase $\exp (i \Theta)$ (dependiente de las coordenadas espaciales homogéneas y el tiempo) y una amplitud $\hat{\mathbf{q}}$ (dependiente de las coordenadas espaciales inhomogéneas). Es decir, $\tilde{\mathbf{q}}=\hat{\mathbf{q}} \exp (i \Theta)$.

La tabla 1.1 resume los diferentes enfoques de análisis modal de inestabilidad lineal que se obtienen relajando las diferentes restricciones en los mencionados enfoques. El caso más restrictivo se define mediante la denominada suposición de flujo paralelo en la que el flujo base se considera homogéneo en dos de las tres direcciones, en nuestro caso $x$ y $z$. El modelo resultante es el modelo de la ecuación de Orr-Sommerfeld (OSE), también denominado análisis modal local 1 . Un modelo ligeramente menos restrictivo es el de las Ecuaciones de Estabilidad Parabolizadas (PSE) [22], también denominado análisis modal no local, donde se considera una lenta dependencia en una coordenada adicional (representada por $x^{*}$ en la tabla 1.1). El próximo paso en la generalización consiste en relajar la restricción impuesta sobre el flujo base a lo largo de la dirección $x$, permitiéndole tener una dependencia arbitraria. De esta forma, el flujo base y las amplitudes de las perturbaciones dependen de dos coordenadas inhomogéneas, mientras que la fase $\exp (i \Theta)$ solo depende de la coordenada $z$. Este caso se define como análisis BiGlobal de estabilidad. Finalmente, el caso más general es el modelo de estabilidad TriGlobal en el que la dependencia inhomogénea es considerada en todas las coordenadas espaciales. Entre el concepto de análisis TriGlobal y PSE está el análisis PSE-3D que asume la existencia de un estado base que depende ligeramente de una coordenada espacial $x^{*}$ y con una dependencia muy fuerte en las otras dos coordenadas espaciales $x, y$. Por último, habría que añadir que el problema de autovalores tridimensional solo está al alcance de grandes instalaciones de supercomputación siendo tanto más costoso cuanto mayor sea el número de Reynolds.

\footnotetext{
${ }^{1}$ Este análisis se denomina local porque las autofunciones solo tienen una coordenada inhomogénea siendo éstas unidimensionales
} 
Tabla 1.1: Diferentes enfoques de análisis modal en la moderna teoría de estabilidad lineal: local (OSE), no local (PSE) y Global (BiGobal,TriGlobal y PSE-3D).

\begin{tabular}{cccc}
\hline \hline Problema & Flujo base & Perturbación & Fase \\
\hline OSE & $\mathbf{Q}(y)$ & $\hat{\mathbf{q}}(y) \exp \left(i \Theta_{1 D}\right)$ & $\Theta_{1 D}=\alpha x+\beta z-\Omega t$ \\
PSE & $\mathbf{Q}\left(x^{*}, y\right)$ & $\hat{\mathbf{q}}(x, y) \exp \left(i \Theta_{2 D}^{*}\right)$ & $\Theta_{2 D}^{*}=\int_{x_{o}}^{x} \alpha(\xi) d \xi+\beta z-\Omega t$ \\
BiGlobal & $\mathbf{Q}(x, y)$ & $\hat{\mathbf{q}}(x, y) \exp \left(i \Theta_{2 D}\right)$ & $\Theta_{2 D}=\beta z-\Omega t$ \\
TriGlobal & $\mathbf{Q}(x, y, z)$ & $\hat{\mathbf{q}}(x, y, z) \exp \left(i \Theta_{3 D}\right)$ & $\Theta_{3 D}^{*}=-\Omega t$ \\
PSE-3D & $\mathbf{Q}\left(x^{*}, y, z\right)$ & $\hat{\mathbf{q}}\left(x^{*}, y, z\right) \exp \left(i \Theta_{3 D}\right)$ & $\Theta_{3 D}^{*}=\int_{x_{o}}^{x} \alpha(\xi) d \xi-\Omega t$ \\
\hline
\end{tabular}

Como conclusión, la complejidad numérica del problema de autovalores puede ser reducida asumiendo una dependencia homogénea del problema en una o más coordenadas espaciales. Esta simplificación implica un incremento en el número de parámetros del sistema lo cual hace más extenso el problema paramétrico. No obstante, tal reducción es apropiada o no dependiendo del problema a resolver.

\subsection{Inestabilidad de flujos de densidad constante}

Uno de los objetivos de esta tesis es el análisis de estabilidad de flujos viscosos e incompresibles de densidad estratificada. Ejemplos paradigmáticos de este tipo de flujos son los vórtices con distribución de densidad radial y aquellos otros flujos en los que ocurren inestabilidades como la de RayleighTaylor y la de Kelvin-Helmholtz. Como primera aproximación al problema, procede analizar la estabilidad de flujos de densidad constante como, por ejemplo, las capas de cortadura y vórtices de densidad uniforme.

\subsubsection{Flujos de vórtices}

Un vórtice es un flujo en rotación alrededor de un eje. La existencia de vórtices, por ser un fenómeno cotidiano (ciclones, anticiclones, tornados, huracanes, remolinos de agua marina...), siempre ha fascinado a la humanidad. Además, podemos decir que los vórtices existen en un amplio rango de escalas de longitud y de tiempo: desde pequeños remolinos, como los que se generan en las aspas de una hélice, con un diámetro del orden del milímetro o centímetro y con un tiempo de duración de pocos minutos, hasta los gigantescos remolinos, como los de la atmósfera de Júpiter, con diámetros de miles de kilómetros y que existen durante siglos. Los vórtices no suelen 
permanecer en los lugares donde se crean, sino que tienden a interaccionar entre sí y migran a otras regiones por diferentes causas.

El estudio de los vórtices en el campo de la aeronáutica es de especial relevancia. Estos se crean como consecuencia de la estructura del flujo producida en la estela de las alas, dando lugar a vórtices de punta de ala wingtip vortices ${ }^{2}$ que se hacen perceptibles al combinarse con las estelas blancas de condensación del vapor de agua de escape del motor, formando espectaculares torbellinos visibles. Debido al gran tamaño de estos vórtices y a las altas velocidades tangenciales contenidas en ellos, existe un riesgo evidente para una aeronave que vuela detrás de otra, sobre todo en las operaciones de despegue y aterrizaje. Por ello, hay que establecer unas distancias mínimas de separación entre aeronaves.

Asimismo, los vórtices tienen muchas aplicaciones industriales en inyectores (reactores químicos, motores de combustión, dispositivos de aerosol) [23].

Con objeto de modelizar el flujo base de un vórtice de estela, G. K. Batchelor [24] obtuvo en 1964 una solución estacionaria asintótica de las ecuaciones incompresibles de Navier-Stokes que se conoce en la literatura con el nombre de vórtice de Batchelor.

Posteriormente, Lessen et al. [25], 26], mediante una aproximación de flujo paralelo del vórtice de G. K. Batchelor [24], obtuvieron el q-vórtice o también denominado Bqv, es decir, una versión simplificada del vórtice de Batchelor, despreciándose la dependencia del campo del flujo con respecto a la dirección axial. En un sistema de referencia cilíndrico $\left(\mathbf{e}_{\mathbf{r}}, \mathbf{e}_{\theta}, \mathbf{e}_{\mathbf{z}}\right)$, el Bqv tiene el siguiente campo de velocidades radial $u_{r}$, azimutal $u_{\theta}$ (vórtice) y axial $u_{z}($ jet/wake $)$.

$$
\begin{aligned}
& u_{r}=0, \\
& u_{\theta}=\frac{q u_{c} \delta}{r}\left[1-\exp \left(-\frac{r^{2}}{\delta^{2}}\right)\right], \\
& u_{z}=u_{b}+u_{c} \exp \left(-\frac{r^{2}}{\delta^{2}}\right)
\end{aligned}
$$

siendo $u_{c}=u_{a}-u_{b}{ }^{3}$, donde $u_{a}$ y $u_{b}$ son la velocidad axial central y de corriente libre, respectivamente, $\delta$ es el radio del núcleo del vórtice y del jet/wake, y $q$

\footnotetext{
${ }^{2}$ También se les conoce en la literatura como vórtices de estela trailing line vortices.

${ }^{3}$ Este incremento lo tomaremos como velocidad de escala en nuestros cálculos.
} 
el parámetro de swirl ${ }^{4}$ que controla la intensidad del movimiento azimutal comparado con el del flujo axial [27]. Además, dicho parámetro de control $q$ está relacionado con la circulación del vórtice $\Gamma$ mediante la expresión $q=\Gamma / 2 \pi$.

Se ha realizado un esfuerzo considerable en comprender las características de la estabilidad de estos flujos [28], dependiendo de la intensidad del giro y los perfiles de velocidad que pueden ser altamente sensibles a las perturbaciones y dar lugar a la destrucción del vórtice [29], [30].

El análisis y el estudio de los vórtices suele resultar complejo debido al campo de velocidades tridimensional que generalmente llevan asociado.

La estabilidad modal local del Bqv de densidad constante ha sido ampliamente estudiada, estando asociados los modos inestables a una inestabilidad centrífuga (CTI). En este tipo de flujos el rol de la viscosidad es complejo. Aunque se pensaba que la viscosidad tenía un efecto estabilizante sobre la mayoría de los modos no viscosos, Lessen et al. [26], Sterwartson [31, Mayer y Powell [32] mostraron también cómo da lugar a la aparición de modos inestables de diferente naturaleza: modos viscosos. La primera clase de modos viscosos para el Bqv fue descubierta por Khorrami [33, [34]. Estos modos existen para los números de onda azimutales $m=0$ y $m=1$ en un rango limitado del parámetro de swirl q, siendo las tasas de crecimiento de estos modos muy débiles. La segunda clase de modos viscosos existe para números de onda azimutales negativos, y son llamados modos centrales, porque su estructura está localizada dentro del núcleo del vórtice. Así pues, centrándose en esta clase de modos centrales, Le Dizès y Fabre [35] probaron que la mayoría de vórtices con un jet axial son realmente inestables con respecto a perturbaciones viscosas si el número de Reynolds es suficientemente grande. Para ello, realizaron un análisis asintótico de los modos viscosos centrales en un vórtice arbitrario con flujo axial.

Delbende et. al [4] realizaron un análisis detallado de la transición de la inestabilidad convectiva a la absoluta para el Bqv, mediante simulación directa, en función del parámetro característico de flujo externo $a{ }^{5}$ y el parámetro de swirl $q$ para un número de Reynolds fijo $R e=667$. Por otro

\footnotetext{
${ }^{4}$ Podemos de decir que este parámetro es una medida de la intensidad del giro del vórtice.

${ }^{5} \mathrm{El}$ parámetro a mide la relación entre la velocidad axial fuera del jet/wake $u_{b}$ y la diferencia de velocidad entre el centro $u_{a}$ y el exterior $u_{b}$ del mismo. Es decir, $a=$ $u_{b} /\left(u_{a}-u_{b}\right)$. La influencia de este parámetro $a$ no se analiza en esta tesis doctoral y siempre se establecerá en cero, ya que se puede eliminar por la invariancia galileana de las ecuaciones de Navier-Stokes. Por tanto, este parámetro no afectará a la tasa de crecimiento de la inestabilidad, sino tan solo a la frecuencia.
} 
lado, determinaron las tasas de crecimiento de la inestabilidad, así como las frecuencias en función del número de onda axial y para modos con números de onda azimutales $m$ comprendidos entre -1 y -12 siendo $a=0$ y $q=0.8$.

La existencia de una familia de modos viscosos inestables en un Bqv con $q>1.5$ y elevados valores del número de Reynolds $R e$ fue demostrada numéricamente por Fabre y Jacquin [3] mediante un análisis temporal de estabilidad. Asimismo, encontraron que la viscosidad tiene un rol puramente estabilizante para valores pequeños de $R e$, mientras que tiene un efecto desestabilizante para $R e \geq 10^{3}$.

Heaton et al. [36] llevan a cabo un análisis global de la estabilidad de un vórtice tipo Batchelor no paralelo (vórtice real). En este trabajo el flujo base se obtiene mediante simulación numérica como una solución exacta y estacionaria de las ecuaciones de Navier-Stokes. El trabajo de estos autores enfatiza la importancia de utilizar un análisis global de la estabilidad en vórtices reales.

González-Gutiérrez et al. [37] utilizaron una metodología espectral de alto orden adaptada para estudiar la inestabilidad de flujos con vórtices. Validaron sus resultados con el caso de un Bqv aislado de densidad constante con $R e=1200$ y $q=0.8$, planteando el problema desde el punto de vista de un análisis BiGlobal EVP.

P. Billant y F. Gallaire [27] obtuvieron mediante el método WKB expresiones analíticas para las autofunciones y autovalores del problema de inestabilidad centrífuga (CTI) de un jet giratorio de densidad constante, en ausencia de viscosidad, para grandes valores del número de onda total $\kappa=\sqrt{k^{2}+m^{2}}$. Posteriormente, validaron sus expresiones asintóticas de los autovalores mediante un cálculo numérico, obteniendo un buen acuerdo.

\subsubsection{Flujos con capa de cortadura entre corrientes paralelas}

Según Michalke [38], la capa límite que no está acotada por paredes se denomina capa límite libre o simplemente capa de cortadura. Esta aparece en jets (chorros) y wakes (estelas) y un rasgo característico de estos perfiles de velocidad es que tienen puntos de inflexión [39]. En este contexto, utilizando un flujo base en velocidad del tipo tangente hiperbólica, Michalke [38] halló numéricamente los autovalores y autovectores de la ecuación de Rayleigh [5] desde un punto de vista de un análisis temporal de la estabilidad. Asimismo, obtuvo las líneas de corriente y la distribución de la vorticidad. Comprobó que los autovalores obtenidos mostraban un buen acuerdo con los correspondientes a las expresiones analíticas de Drazin y Howard [40] válidas para pequeños números de onda. Sin embargo, los resultados obtenidos 
en [38] no concordaban con los experimentales de Freymuth [41]. Este hecho llevó a Michalke a estudiar de nuevo el problema, pero desde el punto de vista de un análisis espacial de la estabilidad [39] obteniendo así un buen acuerdo con los referidos resultados experimentales, sobre todo en el rango de pequeñas frecuencias.

La inestabilidad de flujos homogéneos con capa de cortadura puede ser interpretada en términos de interacción de dos o más ondas libres en el perfil de velocidad. Así, Holmboe [42] postuló que la interacción resonante entre dos o más ondas lineales, progresivas e interfaciales da lugar a inestabilidades de crecimiento exponencial en capas de cortadura no viscosas idealizadas (definidas con funciones lineales a trozos). En esta línea de razonamiento, este autor demostró que para un flujo homogéneo con una capa de cortadura lineal entre dos corrientes paralelas, la inestabilidad de Kelvin-Helmholtz 6 existente es el resultado de la interacción resonante entre dos ondas de vorticidad situadas en interfases de vorticidad (i.e. zonas en las que existe un cambio brusco de vorticidad). Tales interfases aparecen en la atmósfera y en los océanos.

A lo largo de los años la teoría de Holmboe ha sido revisada y ampliada constituyendo lo que se conoce actualmente como teoría de interacción de ondas (WIT) 7 . Por ello, merece interés citar el trabajo de revisión de Carpenter et al. [44]. Estos autores consideraron que la interacción resonante entre dos ondas interfaciales, en una capa idealizada de cortadura de un flujo homogéneo, se produce cuando estas ondas alcanzan un estado de bloqueo de fase, i.e. están en reposo relativo la una con respecto a la otra. Manteniendo esta configuración de bloqueo de fase, las ondas crecen exactamente igual con un crecimiento exponencial. Si bien es cierto que el análisis de Carpenter et al. 44] era aplicable a capas de cortadura idealizadas, también lo extrapoló a flujos homogéneos con capas de cortadura suaves cuyo perfil de densidad es del tipo tangente hiperbólica. De esta forma, consideró que el máximo y el mínimo de la distribución del gradiente de vorticidad representaban los puntos centrales de localización de dos interfases de vorticidad difusas que interaccionaban de igual manera que las interfases de vorticidad

\footnotetext{
${ }^{6}$ Según A. Guha and and R. Raj [4] la KHI se puede producir tanto en un medio homogéneo como heterogéneo. Por ello, estos autores aplican la denominación KHI homogénea para el caso de un flujo de densidad constante, y KHI heterogénea para un flujo de densidad variable.

${ }^{7}$ La teoría de interacción de ondas puede ser también aplicada a flujos no homogéneos y explicar el mecanismo físico de otras inestabilidades como, por ejemplo, la inestabilidad de Taylor-Caulfield (resulta de la interacción entre dos ondas de gravedad interfaciales) y la instabilidad de Holmboe (se produce por la interacción entre una onda de vorticidad y otra interna de gravedad).
} 
de los perfiles de velocidad definidos a trozos: así se podía explicar la existencia de un máximo en la relación de dispersión y la de un número de onda de corte.

\subsection{Inestabilidad de fluidos de densidad variable}

Cuando un flujo presenta densidad estratificada pueden aparecer, en determinadas situaciones, nuevas inestabilidades que no existirían si la densidad fuese constante. Por todo ello, es de importancia realizar en este apartado un estudio del estado del arte de las inestabilidades más relevantes cuando un flujo presenta estratificación en densidad.

\subsubsection{Inestabilidad de Rayleigh-Taylor}

En 1883, Lord Rayleigh [45] descubrió matemáticamente que cuando un fluido denso de densidad $\rho_{1}$ se sitúa encima de otro de menor densidad $\rho_{2}$, debido a la acción de un campo gravitatorio g (ver Fig. 1.1) se produce un tipo de inestabilidad. Esta se desarrolla cuando la interfase de separación de los dos fluidos sufre una pequeña perturbación que hace que el fluido más denso se desplace hacia abajo por acción de la gravedad y el más ligero suba hacia arriba. Así, para el caso de dos fluidos incompresibles semi-infinitos de densidades $\rho_{1}$ y $\rho_{2}\left(\rho_{1}>\rho_{2}\right)$ separados por una interfase abrupta en la que existe una discontinuidad en la densidad, Lord Rayleigh obtuvo la tasa de crecimiento de la inestabilidad. Dicha tasa viene dada por la expresión $\sigma=$

$\sqrt{A_{\rho} k g}$, siendo $A_{\rho}$ el número de Atwood, que es un parámetro adimensional definido como $A_{\rho}=\left(\rho_{1}-\rho_{2}\right) /\left(\rho_{1}-\rho_{2}\right)$.

Desde el punto de vista energético, esto se puede explicar admitiendo que el sistema se encuentra inicialmente en equilibrio inestable de forma que, a la menor perturbación, se tiende a disminuir la energía potencial del sistema y la capa fluida más densa se moverá hacia abajo a través de la menos densa.

Posteriormente, Sir G. Taylor [46] demostró en 1950 que la inestabilidad se podía extender a fluidos acelerados, independientemente de la causa que provocase la aceleración. Por estas dos contribuciones, a esta inestabilidad se le dio el nombre de inestabilidad de Rayleigh-Taylor (RTI). El primer experimento que validó la teoría fue llevado a cabo por Lewis [47] en 1950.

Esta inestabilidad es de fundamental importancia en multitud de aplicaciones que abarcan muchos campos como la física de la atmósfera [48, geofísica [49], la astrofísica [50], fusión del plasma [51], [52], física del espacio [53], la química [54], etc. 


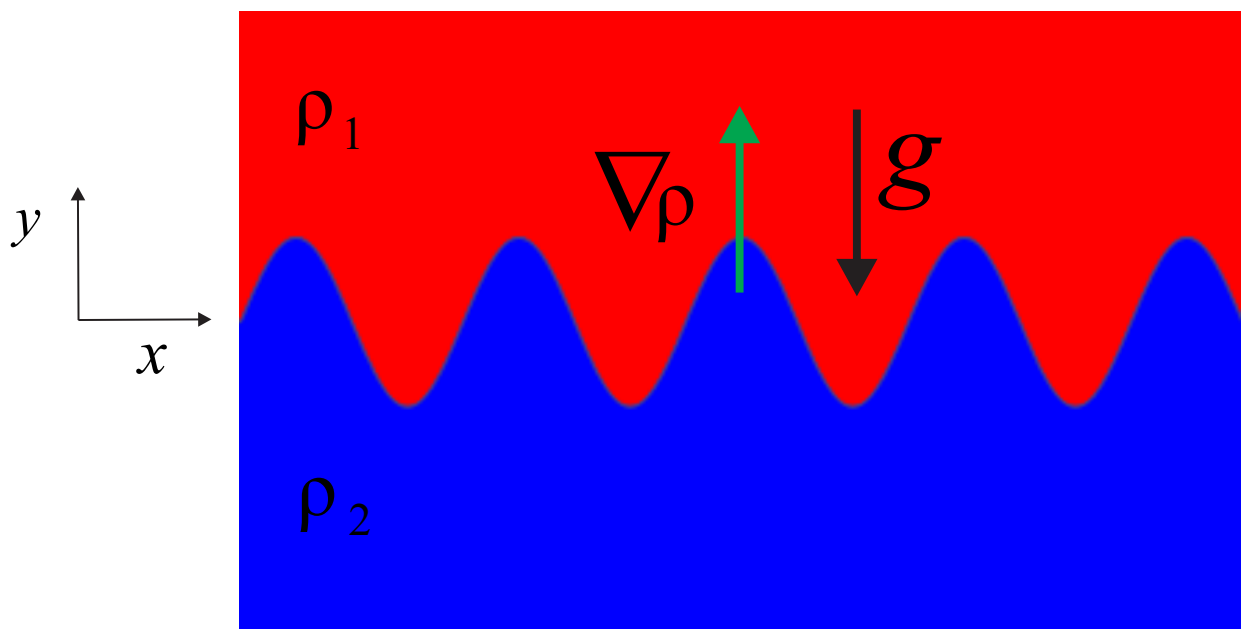

Figura 1.1: Inestabilidad de Rayleigh-Taylor. En nuestro caso $\rho_{1}>\rho_{2} ; \mathbf{g}$ es la intensidad de campo gravitatorio; se puede comprobar que el gradiente de densidad $\nabla \rho$ y $\mathbf{g}$ tienen sentidos opuestos.

El análisis de la RTI para el caso de una capa de transición en densidad difusa entre ambos fluidos 8 suscitó cierto interés en varios autores. Así, Tavakoli et al. [55] analizaron la RTI en una capa de transición en densidad difusa, en ausencia de viscosidad, entre dos fluidos mediante un perfil de densidad del tipo tangente hiperbólica, considerando la existencia de un campo magnético perpendicular a la coordenada espacial de la que depende dicha densidad. Asimismo, su análisis se llevó a cabo para números de Atwood $A_{\rho}$ pequeños $\left(A_{\rho} \ll 1\right)$ y una capa de transición en densidad difusa de anchura $\delta$ grande, de tal forma que $\delta>A_{\rho}$. Obtuvieron soluciones analíticas de un número infinito de modos inestables $(n=0,1 \ldots)$, siendo $n$ el número de orden del modo. Encontraron que dicho campo magnético estabilizaba algunos o todos los modos del espectro de autovalores. Posteriormente, y teniendo en cuenta que el análisis de [55] era solo para $\left(A_{\rho} \ll 1\right)$, Yu et al. [56] investigaron la RTI con una densidad caracterizada, de igual forma, por una función del tipo tangente hiperbólica, pero para $A_{\rho} \leq 0.8$. Obtuvieron una multiplicidad de autovalores, relativos a las tasas de crecimiento de la inestabilidad, y sus correspondientes autofunciones, las cuales tienen po-

\footnotetext{
${ }^{8}$ La densidad del sistema no varía de forma abrupta en la interfase de separación entre ambos fluidos, sino de forma monótona con respecto a una coordenada espacial en la dirección de la aceleración de la gravedad.
} 
tenciales aplicaciones en muchas áreas. Además, obtuvieron una expresión analítica para los autovalores en su forma adimensional, la cual mostraba un buen acuerdo con los resultados numéricos obtenidos por estos autores para números de Atwood $A_{\rho} \leq 0.8$. Posteriormente, con objeto de extender el problema para números de Atwood próximos a la unidad, Dong et al. [57] obtuvieron, además de los autovalores, una expresión analítica para las autofunciones mediante el método de Wentzel-Kramers-Brillouin (WKB).

El efecto de la viscosidad de un fluido sobre la RTI también resultó ser de considerable interés entre los investigadores. Por ello, Chandrasekhar [58] analizó la inestabilidad de Rayleigh-Taylor viscosa (VRTI), es decir, la que presentan los dos fluidos caracterizados por diferentes viscosidades dinámicas, pero en el caso de una transición abrupta. Para el caso particular de idéntica viscosidad cinemática, obtuvo la relación de dispersión para diferentes valores del número de Atwood, mostrando la existencia de un valor máximo de la tasa de crecimiento de la inestabilidad para un número de onda. Comprobó que el valor del máximo de la tasa de crecimiento de la inestabilidad aumentaba con el número de Atwood. Para completar el estudio del efecto de la viscosidad sobre la RTI, surgía la necesidad de investigar el caso de una interfase difusa de separación entre los dos fluidos, ya que los estudios anteriormente mencionados se enmarcaban dentro de un salto de densidad abrupto. Por ello, Morgan et al. [59] estudiaron el comportamiento de la VRTI iniciada con una interfase difusa. Obtuvieron numéricamente, mediante el método de Riccati, la relación de dispersión de un sistema de fluidos superpuestos con densidad y viscosidad variables con respecto a una coordenada espacial. Los resultados mostraron buen acuerdo con los obtenidos por el modelo de Duff et al. 60] para perturbaciones con números de onda pequeños, pero mostraron mayores tasas de crecimiento para perturbaciones con números de onda grandes.

Dado que la RTI implica la existencia de una interfase entre los dos fluidos, un efecto a estudiar sería la importancia, en términos de estabilidad, de la tensión superficial existente en dicha interfase. En cuanto al origen de dicha tensión superficial, podemos decir que la interfase de dos líquidos diferentes e inmiscibles se caracteriza por tener una densidad de energía que está relacionada con la asimetría de las fuerzas intermoleculares y que actúan sobre las moléculas de esta interfase. Cuando el área interfacial se incrementa, hay que suministrar energía externa para llevar moléculas hacia la interfase. Una forma equivalente de analizar la densidad de energía interfacial es a través de la tensión superficial que actúa en dirección tangente a la superficie, con objeto de minimizar su área [61. Dicho todo esto, Bellman and Pennington [62] generalizaron el problema de Rayleigh-Taylor 
incluyendo tensión superficial en la superficie libre de separación entre ambas regiones fluidas. Según estos autores, la presencia de tensión superficial inhibe el proceso de crecimiento de la inestabilidad. Además, para el caso de líquidos incompresibles existe un número de onda de corte $k_{c}$ de tal manera que la superficie de discontinuidad es estable para $k>k_{c}$, es decir, la tensión superficial estabiliza las perturbaciones con un número de onda superior al número de onda de corte.

El efecto de la tensión superficial sobre los fluidos multicapa fue analizado por Mikaelian [63. Este autor estudió también en el mismo trabajo la inestabilidad de Richtmyer-Meshkov. Analizó el caso general de N fluidos con arbitrarias densidades y tensiones superficiales, y derivó la correspondiente ecuación de autovalores que proporciona la tasa de crecimiento de la inestabilidad de las perturbaciones. Para el caso de un sistema de $N=2$, obtuvo la siguiente expresión de la tasa de crecimiento de la RTI

$$
\frac{\sigma^{2}}{g k}=\frac{\rho_{1}-\rho_{2}-k^{2} T_{s} / g}{\rho_{1} \operatorname{coth}\left(k H_{1}\right)+\rho_{2} \operatorname{coth}\left(k H_{2}\right)}
$$

siendo $T_{s}, g$, la tensión superficial y la aceleración de la gravedad, respectivamente. Aquí $H_{1}, \rho_{1}$ y $H_{2}, \rho_{2}$, representan el espesor y la densidad del fluido denso y ligero, respectivamente. Además, Mikaelian [63] estudió la turbulencia en la interfase entre dos fluidos con tensión superficial y presentó predicciones específicas de la energía del movimiento turbulento en función de la tensión superficial.

Partiendo del trabajo de Chandrasekhar [58] parece necesario continuar el análisis del efecto combinado de la tensión superficial y la viscosidad de dos fluidos superpuestos. Por tanto, Reid [64], analizando dichos efectos combinados, realizó un estudio profundo sobre los efectos derivados de la tensión superficial. Encontró que, para un número de Atwood igual a la unidad, el número de onda de corte $k_{c}$ es independiente de la viscosidad cinemática. En ausencia de tensión superficial y de viscosidad la tasa de crecimiento de la inestabilidad crece indefinidamente con el número de onda. Daly [65] realizó un análisis numérico del impacto de la tensión superficial en el crecimiento del spike generado en la inestabilidad de Rayleigh-Taylor.

El efecto combinado del espesor finito y la viscosidad de los dos fluidos sobre la RTI fue analizado por Mikaelian [1]. Este autor obtuvo una relación de dispersión numérica para diferentes espesores y números de Atwood. También comparó sus resultados con los obtenidos para el caso de fluidos semi-infinitos analizado por Chandrasekhar [58], poniendo de manifiesto que el efecto de espesor finito estabilizaba la RTI con respecto al caso estudiado en [58]. 
En lo que a turbulencia se refiere, cabe citar el trabajo desarrollado por Chertkov, Kolokolov y Lebedev [66], quienes investigaron el efecto de la tensión superficial sobre una zona de turbulencia afectada por la RTI. Estos investigadores describieron la estructura interna de una zona turbulenta, producida como resultado del empuje del fluido de mayor densidad sobre el de menor densidad, para el caso de fluidos inmiscibles.

Modelizar una de las regiones fluidas mediante un perfil puramente exponencial también fue objeto de estudio. Así, Obied Allah [67] investigó la RTI lineal de una estratificación constituida por dos fluidos incompresibles de viscosidad despreciable, uno de ellos con una densidad exponencial creciente, soportado por un fluido de densidad constante en presencia de tensión superficial y paredes rígidas. Analizó algunos casos en particular, entre ellos el de un sistema de dos fluidos homogéneos, sin tensión superficial e idéntico espesor. Para este caso, obtuvo que la tasa de crecimiento de la inestabilidad venía dada por

$$
\frac{\sigma^{2}}{g k}=A_{\rho} \tanh (k H)
$$

Esta expresión se puede deducir de 1.9 considerando $T_{s}=0, H=H_{1}=$ $\mathrm{H}_{2}$. Obied Allah [67] encontró que la tensión superficial y las paredes rígidas tienen un efecto estabilizante, mientras que la estratificación debida a zona de densidad exponencial tiene un efecto desestabilizante.

La RTI también puede darse en el caso de que el sistema de los dos fluidos de densidades $\rho_{1}$ y $\rho_{2}\left(\rho_{1}>\rho_{2}\right)$ se encuentre en rotación con una velocidad angular axial $\boldsymbol{\Omega}$. Como puede observarse en la figura 1.2 , y desde el punto de vista de un sistema de referencia ubicado en el propio fluido, aquí el rol de la aceleración de la gravedad lo cumpliría la aceleración centrifuga de inercia (la denominaremos aceleración de la gravedad efectiva $\mathbf{g}_{\mathbf{e f}}$ ), siendo ésta de sentido opuesto al gradiente de densidad $\nabla \rho$. El efecto de la rotación sobre el comportamiento de la RTI ha sido ampliamente analizado siendo combinado con otros efectos como tensión superficial, viscosidad y espesor finito. En esta línea, cabría citar a Vaghela y Chhajlani [68, quienes investigan la RTI en la superficie de separación de dos fluidos parcialmente ionizados que se encuentran superpuestos a través de un medio poroso. En su estudio, estos autores tienen en cuenta los efectos de un campo magnético variable horizontal, tensión superficial y rotación a lo largo de un eje vertical. Concluyen que el sistema es inestable solamente para aquellos números de onda positivos inferiores a un cierto valor crítico en el caso de un gradiente adverso de densidad. Estos estudios también se llevaron a cabo con sistemas de tres fases, ver El-Ansary et al. [69], los cuales discutieron teóricamente 
los efectos de la tensión superficial y rotación uniforme a través de un eje vertical para un sistema de tres fluidos. Los fluidos eran considerados incompresibles y con densidad variable. Obtuvieron resultados numéricos para un sistema de dos y tres fluidos, concluyendo que cuando las dos superficies de separación tienen tensiones superficiales distintas de cero, las perturbaciones de pequeña longitud de onda son completamente estabilizadas. Cabe mencionar también el trabajo de Sharmaa et al. [70] quienes estudiaron numéricamente la RTI lineal de dos fluidos superpuestos en presencia de una pequeña rotación, tensión superficial y con partículas de polvo suspendidas. El efecto de las partículas suspendidas, que se consideran esféricas, se añade a la ecuación de la conservación del momento lineal mediante un término dependiente de la velocidad relativa de las partículas con respecto al fluido. Para ello, utilizaron el método de los modos normales con condiciones de contorno apropiadas. Encontraron, entre otros, que la tasa de crecimiento de la inestabilidad depende de la concentración de masa y de la frecuencia de relajación de las partículas de polvo suspendidas. La pequeña rotación, frecuencia de relajación y concentración de masa tienen un efecto estabilizante sobre la tasa de crecimiento de la inestabilidad. Además, determinaron que el número de Atwood tiene una influencia desestabilizante sobre la RTI.

Otro efecto que había que tener en cuenta en el desarrollo de la RTI era el de la compresibilidad del fluido. Por tanto, Ribeyre et al. [71], con objeto de analizar el papel de la RTI en los restos de una supernova, estudiaron los efectos de la compresibilidad sobre la inestabilidad de Rayleigh-Taylor. Al establecer una comparación entre el caso compresible e isotermo con el caso de la RTI clásica (densidad constante para ambos fluidos), comprobaron que la compresibilidad disminuye la tasa de crecimiento de la inestabilidad para longitudes de onda superiores a una longitud de onda crítica. Por otro lado, llegaron a la conclusión de que la compresibilidad aumentaba la tasa de crecimiento, para longitudes de onda por encima de la referida longitud de onda crítica, comparando el caso de la RTI clásica con el de un fluido estratificado incompresible. Asimismo, llegaron a la conclusión de que el efecto de la compresibilidad es inversamente proporcional al número de Atwood y es más importante para los casos donde el salto de densidad es moderado. Por otro lado, analizaron la generación de vorticidad en la interfase de separación de ambos fluidos. Por último, concluyeron que, para varias configuraciones de fluidos, el efecto de la compresibilidad puede ser significativo en restos de supernovas (SNR). Por otro lado, Livescu [72], teniendo en cuenta, además, el efecto de la tensión superficial, estudió los efectos de la compresibilidad sobre las tasas de crecimiento de la inestabilidad de Rayleigh-Taylor en régimen lineal entre dos fluidos inmiscibles. Determinó que el número de onda 


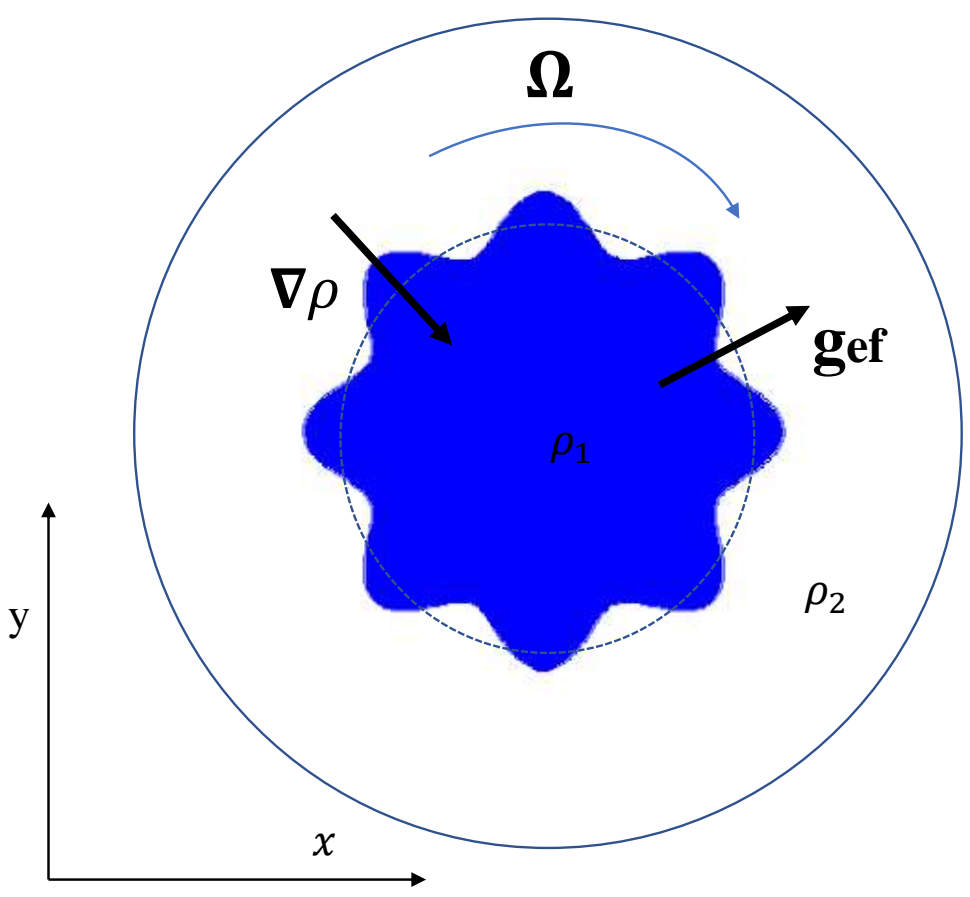

Figura 1.2: Inestabilidad de Rayleigh-Taylor en un sistema de dos fluidos en rotación de densidades $\rho_{1}$ y $\rho_{2}$ y con velocidad angular axial $\boldsymbol{\Omega}$. En nuestro caso $\rho_{1}>\rho_{2} ; \mathbf{g}_{\text {ef }}$ es la aceleración de la gravedad efectiva (aceleración centrífuga de inercia). Se puede comprobar que $\nabla \rho$ y $\mathbf{g}_{\text {ef }}$ tienen sentidos opuestos. 
de corte debido a la presencia de la tensión superficial no se altera por el efecto de la compresibilidad. Asimismo, demostró que para dominios infinitos las tasas de crecimiento de la inestabilidad, obtenidas para el caso compresible, son mayores que las obtenidas para fluidos incompresibles con densidad estratificada exponencialmente y que este hecho no se ve afectado por la presencia de tensión superficial o viscosidad. Otros investigadores, como Lafay et. al [73], se centraron en estudiar los efectos de la compresibilidad sobre la RTI entre dos fluidos miscibles y con viscosidad. Encontraron que un incremento de la estratificación siempre estabiliza la RTI. Por otro lado, comprobaron que las tasas de crecimiento de la inestabilidad se incrementan cuando la compresibilidad de los fluidos ligero y denso decrecen, es decir, cuando disminuimos el coeficiente adiabático de aquellos.

Todo el estudio de la inestabilidad de Rayleigh-Taylor mencionado anteriormente se ha llevado a cabo utilizando un análisis en modos normales. Sin embargo, existe otro enfoque que consiste en reducir el análisis de la inestabilidad a un problema de valor inicial (IVP) mediante el uso de las transformadas de Laplace en el tiempo. El principal descubrimiento del enfoque del problema de estabilidad de un fluido desde un punto de vista de un IVP es que, además de los autovalores del espectro discreto asociados a los modos normales, existe un espectro continuo de autovalores que el análisis en modos normales no puede proporcionar en forma completa. Así, Case [74 haciendo un análisis de estabilidad desde el punto de vista de un IVP, demostró que las soluciones de las ecuaciones de Navier-Stokes linealizadas tendían a las ecuaciones linealizadas de Euler cuando la viscosidad tendía a cero.

Asimismo, Case [75] investigó la estabilidad de una atmósfera invertida. Demostró que una perturbación inicial crece de similar manera a la encontrada por Taylor para el caso de la inestabilidad de superficies aceleradas. El problema de la estabilidad de una atmósfera idealizada con un flujo constante por cizalla y una densidad exponencial decreciente también fue analizado de nuevo por Case [76]. En su estudio, este autor puso de manifiesto que en ningún caso una perturbación inicial se incrementa con el tiempo. Así, el decrecimiento es, en general, no más rápido que una pequeña potencia fraccionaria del tiempo.

Un IVP de perturbaciones de pequeña amplitud para el flujo atmosférico zonal con cizalladura vertical constante y gradiente de temperatura en disminución, utilizando la teoría geostrófica y la aproximación plano beta, fue resuelto por Burger [77]. Encontró longitudes de onda para las que no existía un modo inestable, sino un espectro continuo inestable. 
En otro orden de cosas, las pequeñas amplitudes electrostáticas en un plasma frío con una densidad continua variable fueron investigadas por Sedlácek [78]. Mostró que las inhomogeneidades del plasma llevan a un amortiguamiento de la inestabilidad sin disipación. Utilizó tanto el análisis en modos normales como el método del problema del valor inicial y mostró que los dos son equivalentes. Sin embargo, puesto que el análisis en modos normales puede dejar pasar por alto la existencia de modos colectivos, concluyó que este método no puede reemplazar la técnica de la transformada de Laplace completamente. Cuando abordó el problema desde el punto de vista del IVP, construyó la función de Green de la ecuación diferencial del problema, poniendo de manifiesto la existencia de puntos de ramificación sobre el eje real del plano complejo de frecuencias. Además, encontró un número infinito de nuevas singularidades (simples polos) de la función de Green en la mitad inferior del plano de frecuencias complejo.

Un análisis de la inestabilidad de fluidos, con perfiles de densidad con decrecimiento lento, sometidos a la acción de un campo gravitatorio fue realizado por Ott y Russell [79]. Estos autores descubrieron que la inestabilidad de Rayleigh-Taylor exhibe diferente comportamiento por debajo y por encima de un determinado número de onda crítico $k_{c}$.

El problema del valor inicial asociado con el desarrollo de perturbaciones de pequeña amplitud debidas a una inestabilidad de Rayleigh-Taylor para fluidos viscosos e incompresibles fue analizado por Menikoff et al. [80]. Además de encontrar un espectro discreto de autovalores, descubrieron un espectro continuo, no tratado con anterioridad en la literatura, el cual realiza una importante contribución al desarrollo del movimiento del fluido.

El comportamiento de pequeñas perturbaciones en una capa límite de un fluido fue analizado por Gustavsson [81] mediante un planteamiento tipo IVP. Encontró que la evolución de una perturbación viene dada por la existencia tanto del espectro discreto como del continuo.

Similarmente, Russell y Ott 82 analizaron la RTI lineal en la ionosfera ecuatorial. Para un perfil de densidad puramente exponencial, concluyeron que no existen modos inestables. Para ello, resolvieron el problema del valor inicial para la ecuación linealizada del movimiento de los iones en el límite asintótico de tiempos grandes.

Finalmente, Bogdan y Cally [83] consideraron las oscilaciones lineales de una atmósfera plana semi-infinita eléctricamente conductora, con un gradiente constante de temperatura, sujeta a una aceleración de la gravedad uniforme y a un campo magnético también uniforme. Los resultados obtenidos muestran que los movimientos atmosféricos con variaciones horizontales de la forma $\exp (i k x)$, con $k$ real, poseen tanto un discreto de autovalores 


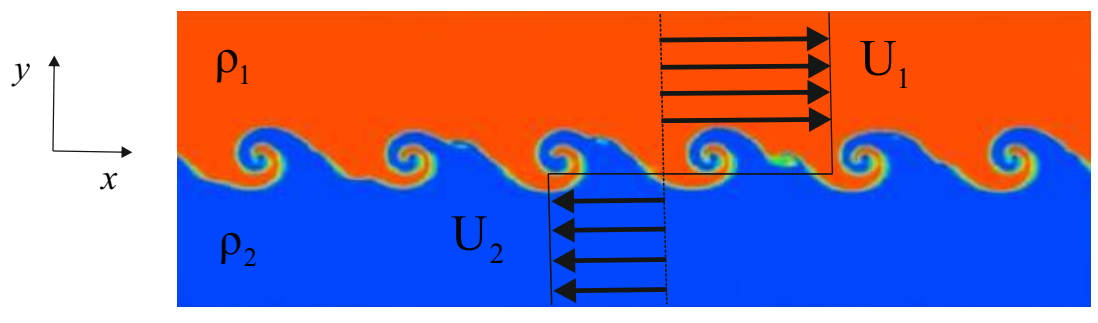

Figura 1.3: Inestabilidad de Kelvin-Helmholtz en un sistema de dos flujos horizontales, paralelos, de diferentes densidades $\rho_{1}$ y $\rho_{2}$ y velocidades $\mathbf{U}_{1}$ y $\mathbf{U}_{2}$. En la interfase de separación entre los dos flujos se observa el inicio de la formación de vórtices.

complejos $\omega_{n}, n=0,1,2 \ldots$ como un espectro continuo.

\subsubsection{Inestabilidad Kelvin-Helmholtz en un medio heterogéneo}

La inestabilidad de Kelvin-Helmholtz (KHI) en un medio heterogéneo se produce cuando, estando dos fluidos inicialmente en movimiento, hay una variación de la componente paralela de la velocidad a través de la interfase [84], [85]. Así, la velocidad de cortadura lleva a la creación de vórtices que penetran en los dos fluidos en un movimiento rotacional muy característico que se puede apreciar en la figura 1.3 .

El mecanismo físico de la KHI fue explicado por H. Von Helmholtz en 1868 [84]. Por otro lado, la formulación matemática de la KHI fue establecida por Kelvin (Sir William Thomson) en 1871 [85].

Según Chandrasekhar [86], para un flujo base caracterizado por los siguientes perfiles de densidad $R(y)$ y velocidad $U(y)$

$$
\begin{gathered}
R(y)= \begin{cases}\rho_{1}, & y>0 \\
\rho_{2}, & y<0\end{cases} \\
U(y)= \begin{cases}U_{1}, & y>0 \\
U_{2}, & y<0\end{cases}
\end{gathered}
$$

la tasa de crecimiento $\sigma_{K H}$ y la frecuencia $\omega_{K H}$ de la inestabilidad de KelvinHelmholtz en régimen lineal vienen dadas por

$$
\begin{gathered}
\sigma_{K H}^{2}=\frac{\rho_{1} \rho_{2} k^{2}\left(U_{1}-U_{2}\right)^{2}}{\left(\rho_{1}+\rho_{2}\right)^{2}} \\
\omega_{K H}=k \frac{\rho_{1} U_{1}+\rho_{2} U_{2}}{\rho_{1}+\rho_{2}}
\end{gathered}
$$


En el caso de coexistencia de RTI, la expresión (1.13) tiene que incluir el término del campo gravitatorio existente $\mathbf{g}=-g \mathbf{j}$ expresándose como

$$
\sigma_{K H-R T}^{2}=\frac{\rho_{1} \rho_{2} k^{2}\left(U_{1}-U_{2}\right)^{2}+k g\left(\rho_{1}^{2}-\rho_{2}^{2}\right)}{\left(\rho_{1}+\rho_{2}\right)^{2}}
$$

Asimismo, este autor explicó el efecto sobre la KHI de la tensión superficial, densidad variable, rotación y campo magnético.

Los primeros estudios experimentales en laboratorio de la KHI fueron realizadas por Francis [87] mediante chorros de aire sobre una capa de aceite muy viscoso en un túnel de viento. Este autor comprobó que la tensión superficial puede ser capaz de inhibir la KHI por debajo de una velocidad relativa umbral de los dos flujos según había predicho con anterioridad Lord Kelvin [85].

La KHI se produce en una amplia variedad de situaciones físicas, siendo la más llamativa la que se produce en la atmósfera terrestre por un cizalladura del viento en condiciones de humedad, generándose nubes muy curiosas en forma de espiral en la vertical. 9

La KHI juega un importante papel en varios fenómenos de la física del espacio y astrofísica, como, por ejemplo, los que ocurren en la interfase del viento solar y la magnetosfera [88 ${ }^{10}$, en la estabilidad de la propagación de chorros [89] y colas de cometas [90]. Asimismo, aparece en determinados fenómenos de la física de alta densidad de energía (HEDP), tales como ondas de choque y jets de altos números de Mach [91]. En el contexto de la Fusión Nuclear por Confinamiento Inercial (ICF), la KHI se puede producir en la microbola de combustible cuando la RTI se ha iniciado: las burbujas que ascienden y los spikes que descienden pueden inducir una cizalladura en la interfase debido a su movimiento, produciendo KHI. Asimismo, la KHI se puede producir en las microbolas por la incidencia oblicua de las ondas de choque 92 .

Los gradientes de densidad y velocidad juegan un papel importante en la KHI. En esta línea de investigación, Wang et al. 93] utilizaron un flujo base en densidad y velocidad construido a base de funciones exponenciales para modelizar una transición difusa en velocidad y densidad. En su estudio analítico utilizaron autofunciones aproximadas y construidas a partir de las correspondientes a la transición abrupta en flujo base, i.e., las de la

\footnotetext{
${ }^{9}$ Este tipo de nubes, producidas por la KHI, son a evitar por los pilotos de aviones, ya que pueden causar turbulencias y mermar el confort de los vuelos.

${ }^{10} \mathrm{La}$ KHI puede dar lugar a que la magnetosfera sea arrastrada por el viento solar con mayor intensidad.
} 
KHI clásica [86 ${ }^{11}$. Asimismo, encontraron analíticamente que el gradiente de densidad i.e., el efecto del espesor finito de la zona de transición del perfil de densidad, desestabiliza la KHI. Este resultado fue validado por Gan et al. [94] mediante una simulación numérica modelizando el flujo base mediante funciones del tipo tangente hiperbólica, utilizado con anterioridad por Faganello et al. 95 para estudiar la KHI en régimen no lineal, y que tenía aparentes similitudes con el flujo base de 93. El estudio de Gan et al. 94 era limitado, ya que su análisis se ciñó exclusivamente al comportamiento del modo $k=10 \pi$ en función de $L_{u}$ y $L_{\rho}$ (las longitudes características de los perfiles de velocidad y densidad, respectivamente) manteniendo fijos el resto de parámetros del sistema, mientras que el de Wang et al.93] permitía obtener la relación de dispersión completa.

Puesto que el efecto combinado de los gradientes de densidad y velocidad estudiado por [93] estaba restringido a fluidos de espesor semi-infinito, Hoshoudy y Cavus [96] lo extendieron para fluidos de espesor finito, ya que el efecto del espesor finito no podía ser obviado en el desarrollo de la KHI en la atmósfera solar [97] y en el viento solar [98]. Estos autores encontraron que la frecuencia normalizada era inferior a la correspondiente al caso de fluidos de espesor semi-infinito. Asimismo, llegaron a la conclusión de que el comportamiento de la tasa de crecimiento de la inestabilidad normalizada en función de los gradientes de velocidad y densidad, para grandes valores del salto de densidad entre el fluido denso y el ligero, se asemeja al efecto estabilizante del gradiente de velocidad.

Fontane y Joly [99] estudiaron la KHI producida en una capa de cortadura entre dos fluidos de diferentes densidades mediante simulación numérica, utilizando en sus condiciones iniciales perfiles de tipo tangente hiperbólica para la densidad y la velocidad. Encontraron modos elípticos e hiperbólicos identificados con anterioridad para una capa de cortadura homogénea. Asimismo, hallaron una segunda clase de modos con tasas de crecimiento de la inestabilidad máxima para un número de onda axial nulo.

La coexistencia de la RTI y KHI ha sido ampliamente investigada. Guzdar et al. [100] estudiaron, mediante un análisis semi-analítico, el efecto sobre la RTI de una velocidad de cortadura del tipo tangente hiperbólica [94, 95] siendo la densidad del flujo una gaussiana. Estos investigadores comprobaron que la velocidad de cortadura tenía un efecto estabilizante sobre la RTI,

\footnotetext{
${ }^{11}$ Las autofunciones aproximadas utilizadas por 93], para la velocidad pertubada en $y$ (coordenada de la inhomogeneidad), son de la forma $v(y)=[\gamma+i k U(y)] e^{-k|y|}$ viniendo dada $U(y)$ por 4.6 y $\gamma=\sigma+i \omega$. En el límite de $L_{u} \rightarrow 0$ y $L_{\rho} \rightarrow 0$, la autofunción se convertiría en la autofunción exacta para el caso de Chandrasekhar [86] de transición abrupta en densidad y velocidad.
} 
produciendo un máximo en la relación de dispersión en el rango de pequeños números de onda y suprimiendo los modos inestables en el rango de grandes números de onda. Posteriormente, Wang et al. [101] estudiaron el efecto de los gradientes de densidad y velocidad en la combinación de la KHI y RTI utilizando el mismo flujo base de [93]. Estos autores encontraron que el efecto del gradiente de densidad estabiliza la RTI, pero desestabiliza la KHI, mientras que el efecto del gradiente de velocidades, i.e. el efecto de espesor finito de la zona de cortadura, tiende a estabilizar la KHI. Asimismo, Ye et al. [102] estudiaron analíticamente la competición entre las RTI y KHI para cocientes arbitrarios de densidad y perfiles continuos de velocidad y densidad. Encontraron que dicha competición entre inestabilidades es dependiente del número de Froude, el cociente de densidades de ambos fluidos y los espesores de las capas de transición de los perfiles de densidad y velocidad.

\subsubsection{La inestabilidad de vórtices con densidad variable}

En el subapartado 1.4.1 habíamos analizado la inestabilidad de un vórtice con densidad constante. Sin embargo, en este apartado nos centraremos en el análisis de la estabilidad de un vórtice con densidad variable. Si un vórtice tiene densidad variable, de forma que el gradiente de densidad tenga sentido opuesto a la aceleración centrífuga, estaremos en una situación donde se puede producir RTI. Por esta razón, es de importancia investigar la estabilidad de estos vórtices no homogéneos en densidad. La densidad del vórtice la podemos expresar mediante un perfil asimétrico, que no es más que una distribución gaussiana de densidad $R(r)$

$$
R(r)=\rho_{b}+\left(\rho_{a}-\rho_{b}\right) \exp \left(-\frac{r^{2}}{\delta_{\rho}^{2}}\right)
$$

siendo $\rho_{a}$ and $\rho_{b}$, respectivamente, las densidades del centro del vórtice y sus alrededores. Además, el radio de esta distribución gaussiana proporciona una segunda longitud característica $\delta_{\rho}$ que estará relacionada con el radio característico $\delta$ del núcleo del vórtice y del jet/wake mediante el parámetro $\epsilon=\delta / \delta_{\rho}$. Así, nos estaremos refiriendo a vórtices de mayor tamaño que los asociados a su campo de densidades cuando $\epsilon>1$. Por otro lado, la relación entre $\rho_{a}$ y $\rho_{b}$ vendrá dada por el contraste de densidad o número de Atwood $A_{\rho}=\left(\rho_{a}-\rho_{b}\right) /\left(\rho_{a}+\rho_{b}\right)$ que será una medida de la inhomogeneidad del vórtice. Nosotros nos centraremos en el caso $0<A_{\rho}<1$ que se corresponde con un vórtice de núcleo de alta densidad [103. El contraste de densidad 
también lo podemos definir en función del cociente de densidades $s=\rho_{a} / \rho_{b}$, expresándose como $A_{\rho}=(s-1) /(s+1)$.

La estabilidad de los vórtices de tipo Lamb-Oseen ha sido ampliamente estudiada. En el trabajo de Joly et al. [104] aparece una descripción pormenorizada de la evolución del estado del arte de este tipo de vórtices. Joly et al. 104 estudiaron la inestabilidad de Rayleigh-Taylor lineal y no lineal en un vórtice asimétrico tipo Lamb-Oseen con densidad variable del tipo (1.16), en el marco de la incompresibilidad, $q=0.5$, en ausencia de viscosidad y despreciando la aceleración de la gravedad. Estos autores explican la existencia de la RTI en un fluido en rotación con un gradiente de densidad mediante la acción de la aceleración centrífuga la cual juega el mismo rol que la aceleración de la gravedad, en el caso común de un fluido denso soportado por uno ligero. Estos autores obtuvieron las frecuencias y las tasas de crecimiento de la inestabilidad para modos con diferentes valores del número de onda azimutal $(m=1, m=2 \ldots)$, así como las estructuras bidimensionales de las autofunciones de la perturbación de la vorticidad. En la misma línea, Sipp et. al [103] estudiaron también el caso de la RTI lineal tratado por [104], pero en más profundidad, considerando una dependencia de las perturbaciones en la dirección axial con un número de onda $k$, poniendo de manifiesto que, además de la RTI que principalmente está asociada a modos con número de onda axial $k=0$ y número de onda azimutal $m \neq 0$ (modos bidimensionales y asimétricos), existe una inestabilidad centrífuga que principalmente afecta a modos $k \neq 0$ y $m=0$ (modos simétricos). Los cálculos los realizaron para un parámetro de swirl $q=1$. También comprobaron que existe una competición entre la RTI y una inestabilidad centrífuga (CTI) solo para altos valores del número de Atwood. De hecho, para pequeños valores del número de Atwood $A_{\rho}$ y longitud característica $\delta_{\rho}$ del perfil gaussiano de la densidad, solo existe RTI, mientras que para pequeños valores del número de Atwood y altos valores de $\delta_{\rho}$, solo existirá CTI. Así, para $A_{\rho}<0.5$, la CTI ocurre solamente para $\delta_{\rho}>1$ y la RTI para $\delta_{\rho} \leq 0.8$.

\subsubsection{La inestabilidad de torbellino de densidad variable con jet axial.}

En el apartado 1.4.1 hemos analizado la estabilidad de un Bqv de densidad constante. Si queremos extender nuestra definición al caso de una densidad variable, esta la podemos expresar mediante el perfil asimétrico (1.16).

Físicamente, para generar un flujo en rotación con densidad variable una posibilidad es comunicar un giro a un jet que tenga una densidad diferente 
Tabla 1.2: Comparativa entre modelos de vórtices

\begin{tabular}{ccccc}
\hline \hline Autores & Vórtice & Densidad & $R e$ & $q$ \\
\hline Delbende et al. (1999) [4] & Bqv & $R=$ cte & 667 & 0.8 \\
Joly et al. $(2005)[104]$ & Lamb-Oseen $k=0$ & $R(r)$ & $\infty$ & 0.5 \\
Sipp et al. $(2005)[103]$ & Lamb-Oseen $k \neq 0$ & $R(r)$ & $\infty$ & 1 \\
González-Gutiérrez et al. $(2010)[37]$ & Bqv & $R=$ cte & 1200 & 0.8 \\
Di Pierro y Abid (2010)[105] & Bqv & $R(r)$ & $\infty$ & $-10-0$ \\
Pierro y Abid (2012)[2] & Bqv & $R(r)$ & 667 & $0.4-1.4$ \\
Pierro y Abid (2012)[106] & Bqv & $R(r)$ & 1000 & -0.5 \\
Billant y Gallaire (2013)[27] & Bqv & $R=c t e$ & $\infty$ & 1 \\
Pierro et al. $(2013)[23]$ & Experimental $k \neq 0$ & $R(r)$ & $2100-3500$ & $0.6-0.99$ \\
\hline
\end{tabular}

a la densidad del ambiente $\rho_{b}[2]$.

La inestabilidad de un Bqv con densidad variable, en ausencia de viscosidad y en régimen lineal, fue estudiada por Di Pierro y Abid [105]. En primer lugar, obtuvieron unas expresiones asintóticas de los autovalores para $k>>1, m>>1$ que incluso mostraban también un aceptable acuerdo para valores pequeños de estos números de onda. Así, validaron dichos autovalores con los obtenidos numéricamente para tres casos diferenciados $(k \neq 0 \mathrm{y}$ $m=0, k=0$ y $m \neq 0, k \neq 0$ y $m \neq 0$ ) obteniendo un buen acuerdo. El efecto de la viscosidad en el vórtice también ha sido ampliamente analizado. Así, Di Pierro y Abid [106] analizaron numéricamente la competición entre la RTI, la inestabilidad de Taylor-Couette y una inestabilidad debida al acoplamiento entre la rotación y la cizalla axial, utilizando dos flujos base: el primero es el correspondiente a un Bqv de densidad variable y el segundo es un flujo de vórtice con flujo axial, medido experimentalmente por Gallaire y Chomaz [107] y extendido al caso de densidad variable. Resolvieron las ecuaciones de Navier-Stokes para un número de Reynolds $R e=1000$ y para $q=-0.5$. Estudiaron la estabilidad del caso lineal y no lineal. Encontraron que cuando el flujo axial interior es más denso que el exterior (un caso favorable para que se dé la RTI gracias a la fuerza centrífuga) la inestabilidad no es RTI dominante. Además, encontraron que cuando se produce RTI, esta es helicoidal. En la misma línea de investigación, Di Pierro y Abid [2] llevaron a cabo un análisis espacio-temporal de la estabilidad de un Bqv incompresible de densidad variable (inhomogéneo) para un $R e=667$ y parámetro de swirl $q=0.8$. Llevaron a cabo un proceso de validación reproduciendo los resultados obtenidos por Delbende et al. [4] para el caso de densidad constante. Además, obtuvieron, para el caso lineal, las tasas de crecimien- 
to de la inestabilidad en función del número de onda axial para modos de números de onda azimutales $m$ comprendidos entre -1 y -10 y un cociente entre la densidad del centro del vórtice y la de los alrededores $s=2$. Asimismo, determinaron unos mapas de contorno de la tasa de crecimiento de la inestabilidad en función de los parámetros $0.4 \leq q \leq 1.4$ y $1 \leq s \leq 2$.

El estudio de la estabilidad lineal de un jet en remolino, a moderados números de Reynolds, fue ampliado con el trabajo de Di Pierro et al. [23]. Estos investigadores utilizaron un flujo base medio, obtenido experimentalmente, que fue ajustado para el campo de velocidades con las expresiones propuestas por [107] y con otra expresión para la densidad variable. Los cálculos los realizaron para números de Reynolds comprendidos entre 2100 y 3500 y para un parámetro de swirl $q$ entre 0.66 y 0.99. También llevaron a cabo una validación numérica de los resultados obtenidos por Delbende et al. 4] para el Bqv de densidad constante tomando $R e=667$ y $q=0.8$.

Un resumen de los casos más relevantes expuestos anteriormente para vórtices de densidad constante y variable se muestra en la tabla 1.2 .

\subsection{Organización de la tesis}

Esta tesis doctoral está dedicada a investigar las inestabilidades RTI, KHI y CTI en régimen lineal para fluidos estratificados. Para ello, en lo que se refiere a la resolución del EVP, se utilizarán métodos numéricos de bajo orden (método de los elementos finitos) y alto orden (método espectral de Chebyshev) 108. La implementación del método numérico de bajo orden se hará a través de un código Fortran y la del método numérico de alto orden mediante un código en Matlab. En cuanto a la resolución del IVP, se utilizará Mathematica para el cálculo de los autovalores y para realizar la inversión numérica de la transformada de Laplace. Por ello, este trabajo está organizado como sigue: en el capítulo 2 describiremos las herramientas matemáticas que vamos a utilizar en el desarrollo del presente trabajo de investigación. En el capítulo 3 analizaremos, tanto desde el punto de vista de una geometría unidimensional 1D como bidimensional 2D, la RTI de un fluido viscoso regularizado. En el capítulo 4 estudiaremos desde el punto de vista numérico y lineal la KHI en un medio heterogéneo. Para ello, regularizaremos los saltos en densidad y velocidad del flujo base mediante funciones del tipo tangente hiperbólica [94, 95]. El estudio de estabilidad se realizará para geometrías 1D y 2D. En el capítulo 5 llevaremos a cabo un análisis BiGlobal de la inestabilidad de vórtices de densidad variable con objeto de complementar los trabajos de Joly et al. [104] (RTI) y Di Pierro y Abid [2] 
(CTI). Asimismo, en el capítulo 6 investigaremos la RTI en la superficie libre de separación de dos fluidos no viscosos, incompresibles y semi-infinitos: uno cuya densidad crece exponencialmente que estará soportado por otro de densidad constante, en presencia de un campo gravitatorio. A diferencia de los capítulos 3 5, resolveremos el problema de la estabilidad del sistema de los dos fluidos desde el punto de vista de un problema de valor inicial (IVP). Por último, en el capítulo 7 se mostrará un resumen de las conclusiones obtenidas en los capítulos 36 y se mencionarán posibles líneas futuras de investigación. 

CAPÍtulo 2

\section{Metodología. Herramientas matemáticas}

En este capítulo estableceremos las bases matemáticas de la presente tesis doctoral. En primer lugar, describiremos el método de análisis en modos normales y su aplicación a las ecuaciones de Navier-Stokes linealizadas (LNSE) bajo distintas hipótesis. En segundo lugar, analizaremos la técnica de la transformada de Laplace y su utilidad en el estudio de la estabilidad de un flujo como problema de valor inicial (IVP).

\subsection{Las ecuaciones de Navier-Stokes}

Las conocidas ecuaciones de Navier-Stokes (NSE) describen razonablemente la dinámica de un fluido viscoso y multifásico. Dichas ecuaciones fueron propuestas inicialmente por Henri Navier en 1822 y por Simeon Denis Poisson en 1829 basándose en un modelo molecular de los fluidos y, más tarde, por George Gabriel Stokes en 1845 sin necesidad de ninguna hipótesis microscópica [109]. Estas ecuaciones de Navier-Stokes se han desarrollado bajo la hipótesis de que el fluido viscoso que se estudia es un medio continuo y que existe equilibrio termodinámico total.

Las NSE constituyen un sistema de ecuaciones diferenciales en derivadas parciales (PDE) no lineales. Por tanto, no tienen soluciones analíticas conocidas y la necesidad de una resolución numérica de las mismas resulta evidente, mejorando al paso de los años las soluciones obtenidas tanto en tiempo como en precisión.

En nuestro estudio, partiremos de las ecuaciones de Navier-Stokes en su forma dimensional para un flujo de densidad variable y en la notación indicial de Einstein, también utilizada por Chandrasekhar [86]. Así, la ecuación de conservación del momento lineal se puede expresar como

$$
\rho \frac{\partial u_{i}}{\partial t}+\rho u_{j} \frac{\partial u_{i}}{\partial x_{j}}=-\frac{\partial p}{\partial x_{i}}+\frac{\partial}{\partial x_{j}}\left[\mu\left(\frac{\partial u_{i}}{\partial x_{j}}+\frac{\partial u_{j}}{\partial x_{i}}\right)\right]+\rho g_{i}
$$


En segundo lugar, como la densidad del fluido es variable y este es incompresible, la derivada material de la densidad ha de ser cero ${ }^{1}$

$$
\frac{\partial \rho}{\partial t}+u_{j} \frac{\partial \rho}{\partial x_{j}}=0
$$

Por último, la tercera ecuación del problema será la ecuación de la conservación de la masa que para un fluido incompresible se puede expresar

$$
\frac{\partial u_{i}}{\partial x_{i}}=0
$$

donde $u_{i}(i=1,2,3)$ denotan las componentes de la velocidad. Además, $p$, $\rho$ y $t$ son la presión, densidad y el tiempo, respectivamente y, por último, $g_{i}$ representa las fuerzas por unidad de masa en la dirección de la aceleración de la gravedad.

Cuando la viscosidad dinámica del fluido $\mu$ es nula o despreciable (no existe rozamiento interno en el fluido ni de éste con el contorno), las ecuaciones de Navier-Stokes 2.1) y (2.2) se transforman en las ecuaciones de Euler, que en el caso de densidad variable son

$$
\begin{gathered}
\rho \frac{\partial u_{i}}{\partial t}+\rho u_{j} \frac{\partial u_{i}}{\partial x_{j}}=-\frac{\partial p}{\partial x_{i}}+\rho g_{i} \\
\frac{\partial \rho}{\partial t}+u_{j} \frac{\partial \rho}{\partial x_{j}}=0 \\
\frac{\partial u_{i}}{\partial x_{i}}=0
\end{gathered}
$$

Las ecuaciones (2.4)-(2.6) rigen el movimiento de un fluido ideal.

A continuación, procede adimensionalizar las ecuaciones (2.1), (2.2) y (2.3) en función de magnitudes características de tiempo $t_{c}$, longitud $l_{c}$, velocidad $u_{c}$, presión $p_{c}$, densidad $\rho_{c}$, viscosidad dinámica $\mu_{c} \mathrm{y}$ viscosidad cinemática $\nu_{c}$. Así, podemos definir las siguientes magnitudes adimensionales de tiempo $\bar{t}=t / t_{c}$, longitud $\bar{x}_{i}=x / l_{c}(i=1,2,3)$, velocidad $\bar{u}_{i}=u_{i} / u_{c}$ $(i=1,2,3)$, presión $\bar{p}=p / p_{c}$, densidad $\bar{\rho}=\rho / \rho_{c}$ y viscosidad dinámica $\bar{\mu}=\mu / \mu_{c}$.

\footnotetext{
${ }^{1}$ Como el flujo es incompresible, la densidad de cada partícula fluida en su movimiento a lo largo del tiempo ha de permanecer constante.
} 
De esta forma las ecuaciones (2.1), 2.2) y 2.3 normalizadas podrían expresarse como

$$
\begin{gathered}
\bar{\rho} \frac{\partial \bar{u}_{i}}{\partial \bar{t}}+\bar{\rho} \bar{u}_{j} \frac{\partial \bar{u}_{i}}{\partial \bar{x}_{j}}=-\frac{\partial \bar{p}}{\partial \bar{x}_{i}}+\frac{\partial}{\partial \bar{x}_{j}}\left[\bar{\mu}\left(\frac{\partial \bar{u}_{i}}{\partial \bar{x}_{j}}+\frac{\partial \bar{u}_{j}}{\partial \bar{x}_{i}}\right)\right]+\bar{\rho} \lambda_{i} \\
\frac{\partial \bar{\rho}}{\partial \bar{t}}+\bar{u}_{j} \frac{\partial \bar{\rho}}{\partial \bar{x}_{j}}=0 \\
\frac{\partial \bar{u}_{i}}{\partial \bar{x}_{i}}=0
\end{gathered}
$$

donde $\lambda_{i}$ es el vector unitario en la dirección de la aceleración de la gravedad. No obstante, y por cuestiones prácticas a efectos de notación y sin pérdida de generalidad, en los siguientes subapartados prescindiremos del símbolo de barra para representar magnitudes adimensionales.

\subsection{Análisis modal de las LNSE}

Como hemos visto en el capítulo anterior, el problema general de autovalores 1.7 de las LNSE es tridimensional, ya que las perturbaciones son inhomogéneas en las tres direcciones $x, y, z$. Por ello, en un principio estaríamos ante un análisis lineal TriGlobal de la inestabilidad [16]. Sin embargo, este problema de autovalores tridimensional solo está al alcance de grandes instalaciones de supercomputación, siendo tanto más costoso cuanto mayor sea el número de Reynolds. Por ello, dentro del marco de los diferentes tipos de análisis modal mencionados en el capítulo anterior (ver tabla 1.1), en este trabajo nos centraremos en dos enfoques de analisis modal para abordar el problema de la inestabilidad lineal de un flujo: un análisis BiGlobal(2D) y un análisis local de la inestabilidad (1D).

Como conclusión, la complejidad numérica del problema de autovalores puede ser reducido asumiendo una dependencia homogénea del problema en una o más coordenadas espaciales. Esta simplificación implica un incremento en el número de parámetros del sistema lo cual hace más extenso el problema paramétrico. No obstante, tal reducción es apropiada o no dependiendo del problema a resolver

\subsubsection{Análisis BiGlobal de la inestabilidad: LNSE en dos dimensiones (2D)}

En nuestro análisis BiGlobal vamos a relajar la restricción impuesta sobre el flujo base $\mathbf{Q}$ a lo largo de la dirección $x$, permitiendo tener una depen- 
dencia arbitraria. Así, el flujo base de igual forma que las amplitudes de las perturbaciones, va a depender de dos coordenadas inhomogéneas $x$ e $y$. Por el contrario, en la dirección $z$ se permite que las perturbaciones tengan una expansión armónica. Dicho lo cual, este caso se denomina aproximación de estabilidad BiGlobal [110], [111]. Así, la descomposición de la solución de las ecuaciones de Navier-Stokes $\mathbf{q}(x, y, z, t)$ que puede llevarse a cabo sería

$$
\mathbf{q}(x, y, z, t)=\mathbf{Q}(x, y)+\epsilon \tilde{\mathbf{q}}(x, y, z, t), \epsilon<<1
$$

Teniendo en cuenta lo dicho anteriormente, las perturbaciones $\tilde{\mathbf{q}}$ se pueden expresar como

$$
\tilde{\mathbf{q}}(x, y, z, t)=\hat{\mathbf{q}}(x, y) \exp \left(i k_{z} z+\gamma t\right)
$$

Es decir, el problema queda reducido a un dominio bidimensional, que se extiende periódicamente en $z$ caracterizado por la longitud de onda o longitud de periodicidad $L_{z}$ asociada al número de onda real $k_{z}$.

En este nuevo marco teórico, identificaremos la dirección vertical con el eje $y$ de forma que el flujo base pueda contener tres velocidades en las tres direcciones del espacio $U_{i}(i=1,2,3)$ siendo $U_{i}(x, y)$ (flujo base bidimensional), sin necesidad de recurrir a la suposición de que las autofunciones tengan periodicidad en la dirección horizontal $x$, asumiendo, por el contrario, una longitud infinita perpendicular al plano del problema $x y$. De esta forma podemos llevar a cabo un análisis BiGlobal de la inestabilidad. En este análisis, consideraremos que las amplitudes de las perturbaciones dependen de dos coordenadas espaciales.

A continuación, vamos a considerar un flujo base estacionario. Este flujo $\left(U_{i}(x, y), R(x, y), P(x, y)\right)$ va a depender de las coordenadas $x$ e $y$, asumiendo la coordenada $z$ como homogénea. Este flujo base lo perturbaremos con perturbaciones de pequeña amplitud en velocidad $\tilde{u}_{i}$, presión $\tilde{p}$ y densidad $\tilde{\rho}$

$$
\begin{gathered}
u_{i}(x, y, z, t)=U_{i}(x, y)+\varepsilon \tilde{u}_{i}(x, y, z, t)+c . c . \\
p(x, y, z, t)=P(x, y)+\varepsilon \tilde{p}(x, y, z, t)+c . c . \\
\rho(x, y, z, t)=R(x, y)+\varepsilon \tilde{\rho}(x, y, z, t)+c . c .
\end{gathered}
$$

Así, podemos perturbar las ecuaciones 2.7), 2.8, 2.9) donde $\varepsilon<<1$ y c.c. denotan los conjugados de las magnitudes complejas $\tilde{u}_{i}, \tilde{p}$ y $\tilde{\rho}$. Cuando las expresiones 2.12, 2.13) y 2.14 son sustituidas en las ecuaciones (2.7), 
(2.8) y (2.9), se tiene en cuenta que el flujo base cumple las ecuaciones estacionarias y despreciando términos de segundo orden, las ecuaciones de Navier-Stokes linealizadas (LNSE) quedarán expresadas como

$$
\begin{aligned}
R \frac{\partial \tilde{u}_{i}}{\partial t}+R U_{i} \frac{\partial \tilde{u}_{i}}{\partial x_{i}}+R \tilde{u}_{i} \frac{\partial U_{i}}{\partial x_{i}}+\tilde{\rho} U_{i} \frac{\partial U_{i}}{\partial x_{i}}=-\frac{\partial \tilde{p}}{\partial x_{i}}+ \\
\mu \Delta u_{i}+\left(\frac{\partial \tilde{u}_{j}}{\partial x_{i}}+\frac{\partial \tilde{u}_{i}}{\partial x_{j}}\right) \frac{\partial \mu}{\partial x_{j}}+\tilde{\rho} \lambda_{i} \\
\frac{\partial \tilde{\rho}}{\partial t}+U_{i} \frac{\partial \tilde{\rho}}{\partial x_{i}}+\tilde{u}_{i} \frac{\partial R}{\partial x_{i}}=0 \\
\frac{\partial \tilde{u}_{i}}{\partial x_{i}}=0
\end{aligned}
$$

A continuación, las perturbaciones pueden escribirse como modos normales de la forma

$$
\begin{gathered}
\tilde{u}_{i}(x, y, z, t)=\hat{u}_{i}(x, y) e^{i k_{z} z+\gamma t}+c . c . \\
\tilde{p}(x, y, z, t)=\hat{p}(x, y) e^{i k_{z} z+\gamma t}+c . c . \\
\tilde{\rho}(x, y, z, t)=\hat{\rho}(x, y) e^{i k_{z} z+\gamma t}+c . c .
\end{gathered}
$$

donde los términos complejos conjugados (c.c.) son necesarios para hacer las perturbaciones reales. De forma adicional, $\gamma=\sigma+i \omega \in \mathbb{C}$ es la frecuencia compleja, siendo $\sigma$ la tasa de crecimiento/decaimiento y $\omega$ la frecuencia de la perturbación, respectivamente. Por otro lado, $k_{z}$ es el número de onda en la dirección del eje z asociado con una longitud de periodicidad $L_{z}=2 \pi / k_{z}$. De esta forma, sustituyendo 2.18, 2.19), 2.20 en las ecuaciones 2.15, 2.16), 2.17), y considerando una dependencia de la viscosidad dinámica del tipo $\mu=\mu(y)$, obtenemos

$$
\begin{aligned}
& R U \partial_{x} \hat{u}+R V \partial_{y} \hat{u}+i k_{z} R W \hat{u}+R U_{x} \hat{u}+R U_{y} \hat{v}+\hat{\rho}\left(U U_{x}+V U_{y}\right)+\partial_{x} \hat{p} \\
& -\mu \Delta \hat{u}-\left(\partial_{x} \hat{v}+\partial_{y} \hat{u}\right) D \mu=-\gamma R \hat{u}
\end{aligned}
$$

$$
\begin{aligned}
& R U \partial_{x} \hat{v}+R V \partial_{y} \hat{v}+i k_{z} R W \hat{v}+R V_{x} \hat{u}+R V_{y} \hat{v}+\hat{\rho}\left(U V_{x}+V V_{y}\right)+\partial_{y} \hat{p} \\
& -\mu \Delta \hat{v}-2 \partial_{y} \hat{v} D \mu+\hat{\rho}=-\gamma R \hat{v}
\end{aligned}
$$




$$
\begin{gathered}
i R U \partial_{x} \hat{w}+i R V \partial_{y} \hat{w}-R k_{z} W \hat{w}+i \hat{\rho}\left(U W_{x}+V W_{y}\right)+i R\left(\hat{u} W_{x}+\hat{v} W_{y}\right) \\
-k_{z} \hat{p}-i \mu \Delta \hat{w}-\left(i \partial_{y} \hat{w}-k_{z} \hat{v}\right) D \mu=-i \gamma R \hat{w} \\
U \partial_{x} \hat{\rho}+V \partial_{y} \hat{\rho}+i k_{z} W \hat{\rho}+R_{y} \hat{v}+R_{x} \hat{u}=-\gamma \hat{\rho} \\
\partial_{x} \hat{u}+\partial_{y} \hat{v}+i k_{z} \hat{w}=0
\end{gathered}
$$

donde

$$
\Delta \hat{w}=\partial_{x}^{2} \hat{w}+\partial_{y}^{2} \hat{w}-k_{z}^{2} \hat{w}
$$

siendo $D=d / d y$ y $\Delta=\partial_{x}^{2}+\partial_{y}^{2}+\partial_{z}^{2}$.

Hasta ahora, las ecuaciones 2.21)- 2.26) representan las LNSE de un flujo monofásico de densidad variable. No obstante, para mayor generalidad sería de interés extrapolar nuestro problema al caso de un flujo bifásico. Por tanto, vamos a considerar a continuación el caso de un flujo bifásico, existiendo una superficie libre de separación entre las dos fases, encontrándose aquella en $y=y_{s}$. Sean $\rho_{1}, \mu_{1}, \nu_{1} \mathrm{y} \rho_{2}, \mu_{2}, \nu_{2}$ la densidad, viscosidad dinámica, viscosidad cinemática de las dos fases a ambos lados de la superficie de separación, $y=y_{s}^{+}$e $y=y_{s}^{-}$, respectivamente, siendo $\rho_{1}>\rho_{2}$. Asimismo, supongamos la existencia de tensión superficial en la superficie libre de separación entre las dos fases fluidas. Así, y de acuerdo con Chandrasekhar [86], bastaría con añadir al primer miembro de la ecuación 2.22 el término

$$
-2\left(\partial_{x x} \hat{\xi}_{s}-k_{z}^{2} \hat{\xi}_{s}\right) S \delta_{s}\left(y-y_{s}\right)
$$

siendo $\hat{\xi}_{s}=\hat{\xi}\left(x, y_{s}\right) e^{i k_{z} z}$ el desplazamiento de la superficie libre ubicada en $y=y_{s}, \delta_{s}\left(y-y_{s}\right)$ la función delta de Dirac y $S$ la tensión superficial adimensional que podemos expresar como

$$
S=\frac{T_{s}}{\left(\rho_{1}+\rho_{2}\right)\left(g \nu^{4}\right)^{1 / 3}}
$$

donde $T_{s}$ es la tensión superficial dimensional.

Para llevar a cabo el proceso de integración numérica, habrá que regularizar la delta de Dirac. Por ello, conviene expresar el término de la tensión 
superficial 2.27) en función de un desplazamiento $\hat{\xi}(x, y, z)$ independiente de $y_{s}^{2}$

$$
-2\left(\partial_{x x} \hat{\xi}-k_{z}^{2} \hat{\xi}\right) S \delta_{s}\left(y-y_{s}\right)
$$

Así, la ecuación (2.22) se transformaría en

$$
\begin{aligned}
& R U \partial_{x} \hat{v}+R V \partial_{y} \hat{v}+i k_{z} R W \hat{v}+R V_{x} \hat{u}+R V_{y} \hat{v}+\hat{\rho}\left(U V_{x}+V V_{y}\right)+\partial_{y} \hat{p} \\
& -\mu \Delta \hat{v}-2 \partial_{y} \hat{v} D \mu+\hat{\rho}-2\left(\partial_{x x} \hat{\xi}-k_{z}^{2} \hat{\xi}\right) S \delta_{s}\left(y-y_{s}\right)=-\gamma R \hat{v}
\end{aligned}
$$

Asimismo, el término $\hat{\xi}$ lo podemos relacionar con la componente $\hat{v}$ de la velocidad como sigue

$$
\hat{v}=\frac{\partial \hat{\xi}}{\partial t}=\gamma \hat{\xi}
$$

De esta forma, podemos plantear un problema generalizado de autovalores para la determinación de $\gamma$. Así pues, las ecuaciones 2.21), 2.23, 2.24, 2.25, 2.30 y 2.31 en su forma matricial quedarían expresadas como

$$
A\left(\begin{array}{c}
\hat{u} \\
\hat{v} \\
\hat{w} \\
\hat{\rho} \\
\hat{\xi} \\
\hat{p}
\end{array}\right)=-\gamma B\left(\begin{array}{c}
\hat{u} \\
\hat{v} \\
\hat{w} \\
\hat{\rho} \\
\hat{\xi} \\
\hat{p}
\end{array}\right)
$$

siendo el operador lineal $A$ la matriz

$$
A=\left(\begin{array}{cccccc}
R U_{x}+\mathcal{L} & R U_{y}-D \mu \partial_{x} & 0 & U U_{x}+V U_{y} & 0 & \partial_{x} \\
R V_{x} & R V_{y}-D \mu \partial_{y}+\mathcal{L} & 0 & U V_{x}+V V_{y}+1 & -2\left(\partial_{x x}-k_{z}^{2}\right) S \delta_{s} & \partial_{y} \\
i R W_{x} & i R W_{y}+k_{z} D \mu & i \mathcal{L} & i\left(U W_{x}+V W_{y}\right) & 0 & -k_{z} \\
R_{x} & R_{y} & 0 & U \partial_{x}+V \partial_{y}+i k_{z} W & 0 & 0 \\
0 & -1 & 0 & 0 & 0 & 0 \\
\partial_{x} & \partial_{y} & i k_{z} & 0 & 0 & 0
\end{array}\right)
$$

\footnotetext{
${ }^{2}$ Esto se puede argumentar desde el punto de vista de una de las propiedades de la delta de Dirac que establece que el producto de una función genérica $\mathrm{h}(\mathrm{x})$ y una delta de Dirac centrada en el punto $a$ se puede expresar como $h(x) \delta(x-a)=h(a) \delta(x-a)[112$.
} 
donde $\mathcal{L}$ es el operador lineal de advección-difusión que vendrá dado por

$$
\mathcal{L}=R U \partial_{x}+R V \partial_{y}+i k_{z} R W-\mu\left(\partial_{x x}+\partial_{y y}-k_{z}^{2}\right)-D \mu \partial_{y}
$$

quedando el operador lineal $B$ expresado mediante la matriz

$$
B=\left(\begin{array}{cccccc}
R & 0 & 0 & 0 & 0 & 0 \\
0 & R & 0 & 0 & 0 & 0 \\
0 & 0 & i R & 0 & 0 & 0 \\
0 & 0 & 0 & 1 & 0 & 0 \\
0 & 0 & 0 & 0 & 1 & 0 \\
0 & 0 & 0 & 0 & 0 & 0
\end{array}\right)
$$

Finalmente, imponiendo las debidas condiciones de contorno de Dirichlet en un determinado dominio el problema quedaría completamente definido.

Caso I. Flujo base viscoso y estratificado con tensión superficial: $R=R(y), \mu=\mu(y), S \neq 0, U=V=W=0$

En este caso vamos a considerar flujo base de velocidad nulo $\left(U_{i}=0\right)$, es decir

$$
U=V=W=U_{x}=U_{y}=V_{x}=V_{y}=W_{x}=W_{y}=0
$$

Asimismo, existe tensión superficial $(S \neq 0)$ y viscosidad dinámica dependiente de la coordenada $y$, i.e., $\mu=\mu(y)$. Además, como dicho análisis de estabilidad tiene lugar en un dominio $2 \mathrm{D}\left(k_{z}=0\right)$ con condiciones de contorno homogéneas tipo Dirichlet en la parte superior e inferior del dominio y condiciones periódicas entre la parte izquierda y derecha de aquel, este marco no contiene cualquier referencia a la coordenada $z$. Por tanto, estamos ante un problema real de autovalores donde la tercera ecuación (2.23), que contiene la componente perturbada de la velocidad $\hat{w}$, puede ser desacoplada del resto.

Sustituyendo estos valores en las ecuaciones (2.21), 2.24), 2.25), 2.30) y (2.31) y considerando, por simplicidad, que la densidad y la viscosidad del flujo base solo dependen de la coordenada espacial y, i.e. $R_{x}=0$, obtenemos las siguientes ecuaciones

$$
\begin{gathered}
\partial_{x} \hat{p}-\mu \Delta \hat{u}-\left(\partial_{x} \hat{v}+\partial_{y} \hat{u}\right) D \mu=-\gamma R \hat{u} \\
\partial_{y} \hat{p}-\mu \Delta \hat{v}-2 \partial_{y} \hat{v} D \mu+\hat{\rho}-2\left(\partial_{x x} \hat{\xi}-k_{z}^{2} \hat{\xi}\right) S \delta_{s}\left(y-y_{s}\right)=-\gamma R \hat{v}
\end{gathered}
$$




$$
\begin{gathered}
R_{y} \hat{v}=-\gamma \hat{\rho} \\
\hat{v}=\frac{\partial \hat{\xi}}{\partial t}=\gamma \hat{\xi} \\
\partial_{x} \hat{u}+\partial_{y} \hat{v}=0
\end{gathered}
$$

Con objeto de simplificar el sistema de cinco a cuatro ecuaciones diferenciales, vamos a eliminar el desplazamiento $\hat{\xi}$ de dicho sistema. Para ello, combinamos 2.39) y 2.40 de forma que

$$
\hat{\xi}=-\frac{\hat{\rho}}{R_{y}}
$$

Por otro lado, consideraremos una delta de Dirac regularizada 3

$$
\delta_{s}\left(y-y_{s}\right)=\frac{R_{y}}{2 A_{\rho}}
$$

siendo $A_{\rho}$ el número de Atwood definido como

$$
A_{\rho}=\frac{\rho_{1}-\rho_{2}}{\rho_{1}+\rho_{2}}
$$

Sustituyendo 2.42 y 2.43 en 2.38, obtenemos

$$
\partial_{y} \hat{p}-\mu \Delta \hat{v}-2 \partial_{y} \hat{v} D \mu+\hat{\rho}+\frac{S}{A_{\rho}} \partial_{x x} \hat{\rho}=-\gamma R \hat{v}
$$

Luego el problema de autovalores queda reducido a un sistema de cuatro ecuaciones diferenciales (2.37), (2.39), 2.41) y (2.45) que expresado en forma matricial sería

$$
A\left(\begin{array}{c}
\hat{u} \\
\hat{v} \\
\hat{\rho} \\
\hat{p}
\end{array}\right)=-\gamma B\left(\begin{array}{c}
\hat{u} \\
\hat{v} \\
\hat{\rho} \\
\hat{p}
\end{array}\right)
$$

\footnotetext{
${ }^{3}$ Sea el perfil de densidad $R=1-A_{\rho}+2 A_{\rho} \theta\left(y-y_{s}\right)$, siendo $\theta\left(y-y_{s}\right)$ la función escalón centrada en $y=y_{s}$ y $A_{\rho}$ el número de Atwood. Puesto que la derivada de la función escalón es la delta de Dirac, i.e., $\delta\left(y-y_{s}\right)=D \theta\left(y-y_{s}\right)$, entonces $R_{y}=2 A_{\rho} \delta\left(y-y_{s}\right)$. Así, $\delta\left(y-y_{s}\right)=R_{y} / 2 A_{\rho}$.
} 
expresándose la matriz $A$, el operador $\mathcal{L}$ y la matriz $B$ por

$$
\begin{gathered}
A=\left(\begin{array}{cccc}
\mathcal{L} & -D \mu \partial_{x} & 0 & \partial_{x} \\
0 & \mathcal{L}-D \mu \partial_{y} & 1+S / A_{\rho} \partial_{x x} & \partial_{y} \\
0 & R_{y} & 0 & 0 \\
\partial_{x} & \partial_{y} & 0 & 0
\end{array}\right) \\
\mathcal{L}=-\mu\left(\partial_{x x}+\partial_{y y}\right)-D \mu \partial_{y} \\
B=\left(\begin{array}{cccc}
R & 0 & 0 & 0 \\
0 & R & 0 & 0 \\
0 & 0 & R & 0 \\
0 & 0 & 0 & 0
\end{array}\right)
\end{gathered}
$$

Caso II. Flujo base viscoso y estratificado sin tensión superficial y velocidad no nula: $R=R(y), \mu \neq 0$ (constante), $S=0, U \neq 0$, $V \neq 0, W \neq 0$

En este caso, vamos a considerar viscosidad dinámica $\mu$ constante y ausencia de tensión superficial $S=0$. En este apartado interesa normalizar las ecuaciones de Navier-Stokes (2.1), 2.2) y (2.3) en función de los números de Reynolds $R e$ y Froude $F_{r}$. Como $\mu$ es constante, las ecuaciones anteriormente mencionadas podrían expresarse

$$
\begin{gathered}
\rho \frac{\partial u_{i}}{\partial t}+\rho u_{j} \frac{\partial u_{i}}{\partial x_{j}}=-\frac{\partial p}{\partial x_{i}}+\mu \frac{\partial}{\partial x_{j}}\left(\frac{\partial u_{i}}{\partial x_{j}}+\frac{\partial u_{j}}{\partial x_{i}}\right)+\rho \lambda_{i} \\
\frac{\partial \rho}{\partial t}+u_{j} \frac{\partial \rho}{\partial x_{j}}=0 \\
\frac{\partial u_{i}}{\partial x_{i}}=0
\end{gathered}
$$

Sea $l_{c}$ la longitud característica del flujo, $t_{c}$ el tiempo característico, $u_{c}$ la velocidad característica, $\rho_{c}$ densidad característica y la presión característica $p_{c}=\rho_{c} u_{c}^{2}$. Por tanto, podemos definir el número de Reynolds $R e$ y el número de Froude $F_{r}$, respectivamente, como $R e=\rho_{c} u_{c} l_{c} / \mu$ y el número de Froude $F_{r}=u_{c} / \sqrt{g l_{c}}$. Así, las ecuaciones 2.50, 2.51, 2.52 normalizadas serán

$$
\bar{\rho} \frac{\partial \bar{u}_{i}}{\partial \bar{t}}+\bar{\rho} \bar{u}_{j} \frac{\partial \bar{u}_{i}}{\partial \bar{x}_{j}}=-\frac{\partial \bar{p}}{\partial \bar{x}_{i}}+\frac{1}{\operatorname{Re}} \frac{\partial}{\partial \bar{x}_{j}}\left(\frac{\partial \bar{u}_{i}}{\partial \bar{x}_{j}}+\frac{\partial \bar{u}_{j}}{\partial \bar{x}_{i}}\right)+\frac{1}{F_{r}^{2}} \bar{\rho} \lambda_{i}
$$




$$
\begin{gathered}
\frac{\partial \bar{\rho}}{\partial \bar{t}}+\bar{u}_{j} \frac{\partial \bar{\rho}}{\partial \bar{x}_{j}}=0 \\
\frac{\partial \bar{u}_{i}}{\partial \bar{x}_{i}}=0
\end{gathered}
$$

No obstante, y por cuestiones prácticas a efectos de notación y sin pérdida de generalidad, prescindiremos del símbolo de barra para representar magnitudes adimensionales.

A continuación, consideremos un flujo base estacionario $\left(U_{i}, R, P\right)$ bidimensional, el cual perturbamos con perturbaciones de pequeña amplitud en velocidad $\tilde{u}_{i}$, presión $\tilde{p}$ y densidad $\tilde{\rho}$.

Cuando las expresiones 2.12 , (2.13) y (2.14) son sustituidas en las ecuaciones (2.53), 2.54) y (2.55), asumiendo que el flujo base es solución del problema estacionario y despreciando términos de segundo orden, obtenemos las ecuaciones linealizadas

$$
\begin{gathered}
R \frac{\partial \tilde{u}_{i}}{\partial t}+R U_{i} \frac{\partial \tilde{u}_{i}}{\partial x_{i}}+R \tilde{u}_{i} \frac{\partial U_{i}}{\partial x_{i}}+\tilde{\rho} U_{i} \frac{\partial U_{i}}{\partial x_{i}}=-\frac{\partial \tilde{p}}{\partial x_{i}}+\frac{1}{\operatorname{Re}} \Delta \tilde{u}_{i}+\frac{1}{F_{r}^{2}} \tilde{\rho} \lambda_{i} \\
\frac{\partial \tilde{\rho}}{\partial t}+\tilde{u}_{i} \frac{\partial R}{\partial x_{i}}=0 \\
\frac{\partial \tilde{u}_{i}}{\partial x_{i}}=0
\end{gathered}
$$

Analizaremos el problema desde el punto de vista de la estabilidad BiGlobal. Para ello, sustituiremos las expresiones de las perturbaciones en la forma de modos normales dadas (2.18), (2.19), 2.20) dentro de las ecuaciones 2.56), 2.57) y 2.58), obteniendo así

$$
\begin{aligned}
R U \partial_{x} \hat{u} & +R V \partial_{y} \hat{u}+i k_{z} R W \hat{u}+R U_{x} \hat{u}+R U_{y} \hat{v}+\hat{\rho}\left(U U_{x}+V U_{y}\right) \\
& +\partial_{x} \hat{p}-\frac{1}{\operatorname{Re}} \Delta \hat{u}=-\gamma R \hat{u} \\
\sigma R \tilde{v}+ & R U \partial_{x} \tilde{v}+R V \partial_{y} \tilde{v}+R W \partial_{z} \tilde{v}+R V_{x} \tilde{u}+R U_{y} \tilde{v}+\tilde{\rho}\left(U V_{x}+V V_{y}\right) \\
= & -\partial_{y} \tilde{p}+\frac{1}{\operatorname{Re}} \Delta \tilde{v}
\end{aligned}
$$




$$
\begin{gathered}
R U \partial_{x} \hat{v}+R V \partial_{y} \hat{v}+i k_{z} R W \hat{v}+R V_{x} \hat{u}+R V_{y} \hat{v}+\hat{\rho}\left(U V_{x}+V V_{y}\right) \\
+\partial_{y} \hat{p}-\frac{1}{\operatorname{Re}} \Delta \hat{v}+\frac{\hat{\rho}}{F_{r}^{2}}=-\gamma R \hat{v} \\
R U \partial_{x} \hat{w}^{*}+R V \partial_{y} \hat{w}^{*}+i k_{z} R W \hat{w}^{*}+i \hat{\rho}\left(U W_{x}+V W_{y}\right)+i R\left(\hat{u} W_{x}+\hat{v} W_{y}\right) \\
-k_{z} \hat{p}-\frac{1}{\operatorname{Re}} \Delta \hat{w}^{*}=-\gamma R \hat{w}^{*} \\
U \partial_{x} \hat{\rho}+V \partial_{y} \hat{\rho}+i k_{z} W \hat{\rho}+R_{y} \hat{v}+R_{x} \hat{u}=-\gamma \hat{\rho} \\
\partial_{x} \hat{u}+\partial_{y} \hat{v}+k_{z} \hat{w}^{*}=0 \\
\Delta \hat{w}^{*}=\partial_{x}^{2} \hat{w}^{*}+\partial_{y}^{2} \hat{w}^{*}-k_{z}^{2} \hat{w}^{*}
\end{gathered}
$$

donde $\hat{w}^{*}$ vendrá dado por $\hat{w}^{*}=i \hat{w}$. De esta forma, el problema generalizado de autovalores para la determinación de $\gamma$ queda expresado como

$$
A\left(\begin{array}{c}
\hat{u} \\
\hat{v} \\
\hat{w}^{*} \\
\hat{\rho} \\
\hat{p}
\end{array}\right)=-\gamma B\left(\begin{array}{c}
\hat{u} \\
\hat{v} \\
\hat{w}^{*} \\
\hat{\rho} \\
\hat{p}
\end{array}\right)
$$

y la matriz $A$ podremos expresarla como

$$
A=\left(\begin{array}{ccccc}
R U_{x}+\mathcal{L} & R U_{y} & 0 & U U_{x}+V U_{y} & \partial_{x} \\
R V_{x} & R V_{y}+\mathcal{L} & 0 & U V_{x}+V V_{y}+F_{r}^{-2} & \partial_{y} \\
i R W_{x} & i R W_{y} & \mathcal{L} & i\left(U W_{x}+V W_{y}\right) & -k_{z} \\
R_{x} & R_{y} & 0 & U \partial_{x}+V \partial_{y}+i k_{z} W & 0 \\
\partial_{x} & \partial_{y} & k_{z} & 0 & 0
\end{array}\right)
$$

siendo el operador $\mathcal{L}$

$$
\mathcal{L}=R U \partial_{x}+R V \partial_{y}+i k_{z} R W-\frac{1}{\operatorname{Re}}\left(\partial_{x x}+\partial_{y y}-k_{z}^{2}\right)
$$


donde la matriz $B$ puede ser expresada como

$$
B=\left(\begin{array}{ccccc}
R & 0 & 0 & 0 & 0 \\
0 & R & 0 & 0 & 0 \\
0 & 0 & R & 0 & 0 \\
0 & 0 & 0 & 1 & 0 \\
0 & 0 & 0 & 0 & 0
\end{array}\right)
$$

Finalmente, imponiendo las debidas condiciones de frontera de Dirichlet en un determinado dominio, el problema quedaría completamente definido.

Caso III. Flujo base no viscoso, estratificado sin tensión superficial y velocidad no nula: $R=R(y), \mu=0, S=0, U \neq 0, V \neq 0, W=0$

En este apartado, además de analizar fluidos carentes de viscosidad, restringiremos nuestro análisis al caso de tensión superficial $S=0$ e inexistencia de componente en $z$ de la velocidad del flujo base $W=W_{x}=W_{y}=0$. Asimismo, no perturbaremos el sistema en la dirección $z$, i.e. $k_{z}=0$. Por todo lo expuesto, la ecuación 2.23) quedaría desacoplada de las ecuaciones (2.21), (2.22), 2.24), 2.25), quedando estas de la forma

$$
\begin{gathered}
R U \partial_{x} \hat{u}+R V \partial_{y} \hat{u}+R U_{x} \hat{u}+R U_{y} \hat{v}+\hat{\rho}\left(U U_{x}+V U_{y}\right)+\partial_{x} \hat{p}=-\gamma R \hat{u} \\
R U \partial_{x} \hat{v}+R V \partial_{y} \hat{v}+R V_{x} \hat{u}+R V_{y} \hat{v}+\hat{\rho}\left(U V_{x}+V V_{y}\right)+\partial_{y} \hat{p}+\hat{\rho}=-\gamma R \hat{v} \\
U \partial_{x} \hat{\rho}+V \partial_{y} \hat{\rho}+R_{y} \hat{v}+R_{x} \hat{u}=-\gamma \hat{\rho} \\
\partial_{x} \hat{u}+\partial_{y} \hat{v}=0
\end{gathered}
$$

que expresadas en forma matricial quedarían

$$
A\left(\begin{array}{c}
\hat{u} \\
\hat{v} \\
\hat{\rho} \\
\hat{p}
\end{array}\right)=-\gamma B\left(\begin{array}{c}
\hat{u} \\
\hat{v} \\
\hat{\rho} \\
\hat{p}
\end{array}\right)
$$


expresándose la matriz $A$, el operador $\mathcal{L}$ y la matriz $B$ por

$$
\begin{gathered}
A=\left(\begin{array}{cccc}
R U_{x}+\mathcal{L} & R U_{y} & U U_{x}+V U_{y} & \partial_{x} \\
R V_{x} & R V_{y}+\mathcal{L} & U V_{x}+V V_{y}+1 & \partial_{y} \\
R_{x} & R_{y} & U \partial_{x}+V \partial_{y} & 0 \\
\partial_{x} & \partial_{y} & 0 & 0
\end{array}\right) \\
\mathcal{L}=R U \partial_{x}+R V \partial_{y} \\
B=\left(\begin{array}{cccc}
R & 0 & 0 & 0 \\
0 & R & 0 & 0 \\
0 & 0 & 1 & 0 \\
0 & 0 & 0 & 0
\end{array}\right)
\end{gathered}
$$

En el caso de que la aceleración de la gravedad no se tuviese en cuenta en nuestro problema como ocurre, por ejemplo, en la inestabilidad de KelvinHelmholtz (KHI), el término $A_{23}$ que es el que contiene la contribución de la aceleración de la gravedad quedaría reducido a $A_{23}=U V_{x}+V V_{y}$.

\subsubsection{Análisis local de la inestabilidad: LNSE en una dimen- sión (1D)}

El caso más restrictivo se define mediante la denominada suposición de flujo paralelo en la cual el flujo base $\mathbf{Q}$ es considerado homogéneo en dos de las tres direcciones espaciales, por ejemplo $x$ y $z$, de tal manera que los coeficientes del problema resultante de autovalores son independientes de $x$ y $z$. Así, la descomposición de $\mathbf{q}(x, y, z, t)$ que puede llevarse a cabo sería

$$
\mathbf{q}(x, y, z, t)=\mathbf{Q}(y)+\epsilon \tilde{\mathbf{q}}(x, y, z, t), \epsilon<<1
$$

siendo $\epsilon<<1$.

Sustituyendo (2.78) en la ecuaciones 2.1), 2.2 y 2.3 y eliminando los términos no lineales, las ecuaciones linealizadas obtenidas de NavierStokes son idénticas a las ecuaciones linealizadas 2.15, 2.16 y 2.17, respectivamente.

Por otro lado, teniendo en cuenta el carácter periódico de las direcciones $x$ e $y$, las perturbaciones se pueden expresar como

$$
\tilde{\mathbf{q}}(x, y, z, t)=\hat{\mathbf{q}}(y) \exp \left[i\left(k_{x} x+k_{z} z\right)+\gamma t\right]
$$


donde $k_{x}$ y $k_{z}$ son los números de onda de las direcciones espaciales $x$ y $z$, respectivamente, definiéndose las correspondientes longitudes de periodicidad como $L_{x}=2 \pi / k_{x}$ y $L_{z}=2 \pi / k_{z}$. Por otro lado, $\gamma$ representa el autovalor complejo en el marco de un análisis temporal.

En esta sección analizaremos, en primer lugar, el caso de viscosidad dinámica variable para posteriormente llevar a cabo un análisis de viscosidad nula.

Caso I. Flujo base viscoso, estratificado con tensión superficial y velocidad nula: $R=R(y), \mu=\mu(y), S \neq 0, U=V=W=0$

Supongamos que nuestro flujo base se caracteriza por tener una viscosidad dinámica y densidad variables, siendo nulo el campo de velocidades. Las ecuaciones linealizadas (2.15), 2.16) y (2.17) se reducirían a las siguientes

$$
\begin{gathered}
R \frac{\partial \tilde{u}_{i}}{\partial t}=-\frac{\partial \tilde{p}}{\partial x_{i}}+\mu \Delta \tilde{u}_{i}+\left(\frac{\partial \tilde{u}_{j}}{\partial x_{i}}+\frac{\partial \tilde{u}_{i}}{\partial x_{j}}\right) \frac{\partial \mu}{\partial x_{j}}+\tilde{\rho} \lambda_{i} \\
\frac{\partial \tilde{\rho}}{\partial t}+\tilde{u}_{i} \frac{\partial R}{\partial x_{i}}=0 \\
\frac{\partial \tilde{u}_{i}}{\partial x_{i}}=0
\end{gathered}
$$

En el análisis local aquí representado siempre vamos a considerar que el flujo es bifásico. Puesto que, además, en dicho análisis local de la inestabilidad las amplitudes de la perturbación solo dependen de una coordenada espacial, el sistema (2.80)-2.82 puede ser simplificado usando las perturbaciones modales de la forma

$$
\begin{aligned}
\tilde{u}_{i}(x, y, z, t) & =\hat{u}_{i}(y) \exp \left(i k_{x} x\right) \exp \left(i k_{z} z\right) \exp (\gamma t) \\
\tilde{\rho}(x, y, z, t) & =\hat{\rho}(y) \exp \left(i k_{x} x\right) \exp \left(i k_{z} z\right) \exp (\gamma t) \\
\tilde{p}(x, y, z, t) & =\hat{p}(y) \exp \left(i k_{x} x\right) \exp \left(i k_{z} z\right) \exp (\gamma t)
\end{aligned}
$$

donde se utilizarán $N$ puntos discretos en el dominio 1D computacional $y \in[-H, H]$.

Asimismo, consideraremos que existe tensión superficial en la superficie libre de separación de las dos fases fluidas ubicada en $y=y_{s}$. Usando el 
ansatz dado por (2.83)-(2.85), las ecuaciones 2.80$)-(2.82)$ pueden ser escritas como

$$
\begin{gathered}
i k_{x} \hat{p}-\mu\left(D^{2}-k^{2}\right) \hat{u}-D \mu\left(i k_{x} \hat{v}+D \hat{u}\right)=-\gamma R \hat{u} \\
D \hat{p}-\mu\left(D^{2}-k^{2}\right) \hat{v}-2 D \mu D \hat{v}+\hat{\rho}+2 k^{2} S \hat{\xi} \delta_{s}\left(y-y_{s}\right)=-\gamma R \hat{v} \\
i k_{z} \hat{p}-\mu\left(D^{2}-k^{2}\right) \hat{w}-D \mu\left(i k_{z} \hat{v}+D \hat{w}\right)=-\gamma R \hat{w} \\
D R \hat{v}=-\gamma \hat{\rho} \\
i k_{x} \hat{u}+i k_{z} \hat{w}=-D \hat{v}
\end{gathered}
$$

siendo $k^{2}=k_{x}^{2}+k_{z}^{2}$ y $D=d / d y$.

El conjunto de ecuaciones (2.86)-2.90 junto con la ecuación

$$
\hat{v}=\frac{\partial \hat{\xi}}{\partial t}=\gamma \hat{\xi}
$$

forman un sistema de seis ecuaciones diferenciales. Con objeto de simplificar el sistema de seis a cinco ecuaciones, procederemos a eliminar el desplazamiento $\hat{\xi}$ de dicho sistema. Para ello, combinamos 2.89 y 2.91. De esta forma

$$
\hat{\xi}=-\frac{\hat{\rho}}{D R}
$$

Sustituyendo 2.92 y la delta de Dirac regularizada dada por la expresión 2.43) en la ecuación (2.87), obtenemos

$$
D \hat{p}-\mu\left(D^{2}-k^{2}\right) \hat{v}-2 D \mu D \hat{v}+\hat{\rho}\left(1-k^{2} S / A_{\rho}\right)=-\gamma R \hat{v}
$$

Posteriormente, multiplicando la ecuación 2.86 por $i k_{x}$ y la ecuación 2.88 por $i k_{z}$, y haciendo uso de la ecuación 2.90 eliminamos componentes $\hat{u}$ y $\hat{w}$ de la velocidad perturbada y obtenemos

$$
k^{2} \hat{p}=\left[-\gamma R+\mu\left(D^{2}-k^{2}\right)\right] D \hat{v}+(D \mu)\left(D^{2}+k^{2}\right) \hat{v}
$$

Usando las ecuaciones 2.89, 2.93 y 2.94, obtenemos un problema de autovalores generalizado reducido a un sistema de tercer orden donde 
las autofunciones son las amplitudes de las perturbaciones de la velocidad vertical $\hat{v}$, densidad $\hat{\rho}$ y presión $\hat{p}$, que expresado en su forma matricial

$$
A\left(\begin{array}{c}
\hat{v} \\
\hat{p} \\
\hat{\rho}
\end{array}\right)=\gamma B\left(\begin{array}{c}
\hat{v} \\
\hat{p} \\
\hat{\rho}
\end{array}\right)
$$

siendo los operadores $A$ y $B$

$$
A=\left(\begin{array}{ccc}
\mu\left(D^{2}-k^{2}\right)+2 D \mu D & -D & -\left(1-k^{2} S / A_{\rho}\right) \\
\mu\left(D^{3}-k^{2} D\right)+D \mu\left(D^{2}+k^{2}\right) & -k^{2} & 0 \\
-D \bar{\rho} & 0 & 0
\end{array}\right)
$$

y

$$
B=\left(\begin{array}{ccc}
R & 0 & 0 \\
R D & 0 & 0 \\
0 & 0 & 1
\end{array}\right)
$$

Este sistema puede ser reducido a un problema de autovalores cuadrático para la velocidad vertical perturbada $\hat{v}$

$$
\left(E \gamma^{2}+F \gamma+G\right) \hat{v}=0
$$

donde los operadores $E, F$ y $G$ son, respectivamente,

$$
\begin{gathered}
E=k^{2} R-D R D-R D^{2} \\
F=\mu\left(D^{4}+k^{4}-2 k^{2} D^{2}\right)+2(D \mu)\left(D^{3}-k^{2} D\right)+\left(D^{2} \mu\right)\left(D^{2}+k^{2}\right) \\
G=-k^{2} D R\left(1-k^{2} S / A_{\rho}\right)
\end{gathered}
$$

La ecuación diferencial de cuarto orden (2.98), en conjunción con sus correspondientes condiciones de contorno, constituye el problema cuadrático de autovalores para $\gamma$. La mencionada ecuación diferencial 2.98) es analizada por Reid [64] para dos fluidos semi-infinitos de diferente densidad e igual viscosidad cinemática; es resuelta por Mikaelian [1] para dos fluidos de espesor finito, de diferente densidad e igual viscosidad cinemática y $S=0$, cuando las condiciones de discontinuidad en densidad, viscosidad dinámica y derivada primera de la velocidad vertical perturbada son impuestas en la superficie libre compartida por ambos fluidos. 
Caso II. Flujo base no viscoso, estratificado, sin tensión superficial y velocidad no nula: $R=R(y), \boldsymbol{\mu}=\mathbf{0}, \boldsymbol{S}=\mathbf{0}, \boldsymbol{U} \neq \mathbf{0}, \boldsymbol{V} \neq \mathbf{0}, \boldsymbol{W}=\mathbf{0}$

En este apartado analizaremos el caso particular de viscosidad y tensión superficial nulas $S=\mu=0$ en la interfase de separación entre ambas fases. Asimismo, consideraremos una componente en $z$ nula del flujo base en velocidad $\left(W=W_{x}=W_{y}=0\right)$. Por tanto, las ecuaciones linealizadas (2.15), 2.16) y 2.17 quedan reducidas a las siguientes

$$
\begin{gathered}
R \frac{\partial \tilde{u}_{i}}{\partial \bar{t}}+R U_{i} \frac{\partial \tilde{u}_{i}}{\partial \bar{x}_{i}}+R \tilde{u}_{i} \frac{\partial U_{i}}{\partial \bar{x}_{i}}+\tilde{\rho} U_{i} \frac{\partial U_{i}}{\partial \bar{x}_{i}}=-\frac{\partial \tilde{p}}{\partial \bar{x}_{i}}+\tilde{\rho} \lambda_{i} \\
\frac{\partial \tilde{\rho}}{\partial \bar{t}}+U_{i} \frac{\partial \tilde{\rho}}{\partial \bar{x}_{i}}+\tilde{u}_{i} \frac{\partial R}{\partial \bar{x}_{i}}=0 \\
\frac{\partial \tilde{u}_{i}}{\partial \bar{x}_{i}}=0
\end{gathered}
$$

Puesto que en un análisis local de la inestabilidad, las amplitudes de la perturbación solo dependen de una coordenada espacial, en nuestro caso la coordenada $y$, el sistema (2.102)-2.104) es ahora simplificado sustituyendo en el mismo las perturbaciones modales dadas por las expresiones 2.83, 2.84) y 2.85

donde han sido utilizados $N$ puntos discretos en el domininio 1D computacional $y \in[-H, H]$.

Usando el ansatz dado por (2.83)-(2.85), las ecuaciones (2.102)-(2.104) pueden ser escritas como

$$
\begin{gathered}
R\left(\gamma+i k_{x} U\right) \hat{u}+R(D U) \hat{v}=-i k_{x} \hat{p} \\
R\left(\gamma+i k_{x} U\right) \hat{v}=-D \hat{p}-\hat{\rho} \\
R\left(\gamma+i k_{x} U\right) \hat{w}=-i k_{z} \hat{p} \\
\left(\gamma+i k_{x} U\right) \hat{\rho}=-\hat{v}(D R) \\
i k_{x} \hat{u}+i k_{z} \hat{w}=-D \hat{v}
\end{gathered}
$$


siendo $k^{2}=k_{x}^{2}+k_{z}^{2}$ y $D=d / d y$. Multiplicando 2.105 por $-i k_{x}$ y 2.107) por $-i k_{z}$ y haciendo uso de 2.109, obtenemos

$$
R\left(\gamma+i k_{x} U\right)(D \hat{v})-i k_{x} R(D U) \hat{v}=-k^{2} \hat{p}
$$

Utilizando las ecuaciones (2.106), 2.108) y 2.110 obtenemos un problema de autovalores generalizado que queda reducido a un sistema de tercer orden, siendo las autofunciones las amplitudes de las perturbaciones de la velocidad vertical $\hat{v}$, presión $\hat{p}$ y densidad $\hat{\rho}$ que expresado en su forma matricial

$$
A\left(\begin{array}{c}
\hat{v} \\
\hat{p} \\
\hat{\rho}
\end{array}\right)=\gamma B\left(\begin{array}{c}
\hat{v} \\
\hat{p} \\
\hat{\rho}
\end{array}\right)
$$

siendo los operadores $A$ y $B$

$$
A=\left(\begin{array}{ccc}
i R k_{x} U & D & 1 \\
i R k_{x}(U D-D U) & k^{2} & 0 \\
D R & 0 & i k_{x} U
\end{array}\right)
$$

y

$$
B=\left(\begin{array}{ccc}
-R & 0 & 0 \\
-R D & 0 & 0 \\
0 & 0 & -1
\end{array}\right)
$$

En el caso de que la aceleración de la gravedad no se tuviese en cuenta en nuestro problema como ocurre, por ejemplo, en la inestabilidad de KelvinHelmholtz (KHI), el término $A_{13}$ que es el contiene la contribución de la aceleración de la gravedad, sería nulo. Por tanto, la matriz $A(2.112$ del problema 2.111) quedaría reducida a

$$
A=\left(\begin{array}{ccc}
i R k_{x} U & D & 0 \\
i R k_{x}(U D-D U) & k^{2} & 0 \\
D R & 0 & i k_{x} U
\end{array}\right)
$$

\subsection{Análisis no modal de estabilidad}

En determinadas situaciones es útil plantear el problema de estabilidad de un sistema de dos fluidos como un problema de valor inicial (IVP). Así, nos aseguramos la inclusión de determinados modos que pertenecen a un espectro continuo, que serían lamentablemente obviados si el análisis de 
estabilidad se realizará desde el punto de vista de un análisis en modos normales. La herramienta fundamental para transformar nuestro problema de estabilidad a un IVP es la transformada de Laplace, que pasaremos a describir a continuación. Finalmente, a modo de ilustración, aplicaremos esta herramienta a un caso particular en el que las ecuaciones de Euler caracterizan un sistema de dos fluidos con tensión superficial.

\subsubsection{La técnica de la la transformada de Laplace}

La transformación de Laplace es una técnica matemática que permite transformar una ecuación diferencial en otra algebraica más sencilla [113. A partir de esta última y mediante una transformación inversa se obtiene la solución completa de la ecuación diferencial de partida, siendo otra de sus ventajas la posibilidad de permitir condiciones iniciales. En definitiva, la transformada de Laplace permite resolver ecuaciones diferenciales lineales formuladas como problemas de valor inicial. A continuación, procederemos a explicar la técnica de una manera concisa. Para determinar la dependencia temporal de una función $f(t)$ que está determinada por una ecuación diferencial lineal, definimos la transformada de Laplace de $f(t)$ como

$$
\mathcal{L}\{f(t)\}=\tilde{f}(s)=\int_{0}^{\infty} f(t) e^{-s t} d t
$$

donde el símbolo $\mathcal{L}$ representa el proceso de transformación. La trasformada de Laplace está definida solamente para la variable compleja $s$ con $\operatorname{Re}(s)$ positiva ${ }^{4} \mathrm{y}$ suficientemente grande, i.e., $\operatorname{Re}(s)>s_{o}$, de tal forma que la integral 2.115) converge en $t \rightarrow \infty$.

$$
\mathcal{L}\left\{f^{\prime}(t)\right\}=s \mathcal{L}\{f(t)\}-f(0)
$$

donde $f(0)$ es el valor de $f(t)$ en $t=0$. De esta forma, la condición inicial es introducida explícitamente dentro de la solución para la transformada de Laplace. Estrictamente hablando, se requiere que $f(0)=f(+0)$ y que $f^{\prime}(t)$ sea al menos continua a trozos (el cero es alcanzado desde el lado positivo) para $0 \leq t<\infty$. Naturalmente, $f(t)$ y su derivada deben ser tales que la integrales no diverjan [114]. Podemos establecer una generalización de forma que

$$
\begin{aligned}
& \mathcal{L}\left\{f^{n}(t)\right\}=s^{n} \mathcal{L}\{f(t)\}-s^{n-1} f(+0)-s^{n-2} f^{\prime}(+0)-\ldots \\
& -f^{n-1}(+0)
\end{aligned}
$$

\footnotetext{
${ }^{4}$ En realidad s es el símbolo de la frecuencia compleja y es igual a $s=\sigma+i \omega$.
} 
Una vez que hemos obtenido $\tilde{f}(s)$ debemos invertir la transformada para encontrar $f(t)$. La fórmula de inversión apropiada es

$$
f(t)=\frac{1}{2 \pi i} \int_{C} e^{s t} \tilde{f}(s) d s
$$

donde $C$ es un camino de integración, con parte real $\operatorname{Re}(s)=s_{o}$, que se extiende desde $-i \infty+s_{o}$ a $i \infty+s_{o}$ lo suficientemente lejos a la derecha del eje imaginario (i.e. un valor $s_{o}$ positivo lo suficientemente grande) para asegurarnos de que todas las singularidades de $\tilde{f}(s)$ queden a la izquierda del contorno, tal y como se muestra en la Fig. 2.1. A la expresión 2.118 se la conoce como la integral de Bromwich 5 , Las singularidades en el semiplano derecho corresponden a términos con crecimiento exponencial $f(t)$ lo cual produce que la integral 2.115 que define $\tilde{f}(s)$ diverja si $\operatorname{Re}(s)$ no es lo suficientemente grande. A menudo tales términos están ausentes, en cuyo caso la elección de un contorno que se extiende a la derecha del eje imaginario en la fórmula de inversión de Laplace es satisfactoria. Como método general, esta integral es evaluada cerrando el contorno $C$ hacia la izquierda. Esto se puede realizar, por ejemplo, añadiendo un arco de circunferencia $C_{R}$ que no contribuya a la integral para $t>0$ (ver figura 2.2). También habría que hacer notar que la integral a través del contorno cerrado debe ser igual $2 \pi i$ veces la suma de los residuos de todas las singularidades dentro del contorno cerrado. En determinados casos, sin embargo, hay un infinito número de singularidades encontradas cuando $\operatorname{Re}(s)=-\infty$ en cuyo caso este planteamiento no daría lugar a una solución en su forma cerrada.

\section{Aplicación a un fluido estratificado con tensión superficial}

Consideremos, a modo de ilustración, un fluido no viscoso de densidad $R(y)$ y presión $P(y)$ en reposo que se encuentra sometido a la aceleración de la gravedad g en la dirección del eje y y sentido negativo. Asimismo, el fluido se va a caracterizar por tener un salto de densidad en una superficie libre ubicada en $y=y_{i}$ sometida a la acción de la tensión superficial. El problema físico está gobernado por las ecuaciones de Euler (2.4), 2.5) y (2.6), que perturbaremos con perturbaciones de pequeña amplitud en velocidad $\tilde{u}_{i}$, presión $\tilde{p}$ y densidad $\tilde{\rho}$

$$
u_{i}(x, y, z, t)=\varepsilon \tilde{u}_{i}(x, y, z, t)+c . c .
$$

\footnotetext{
${ }^{5}$ En la literatura también se la conoce como teorema de Fourier-Mellin o integral de Fourier Mellin.
} 


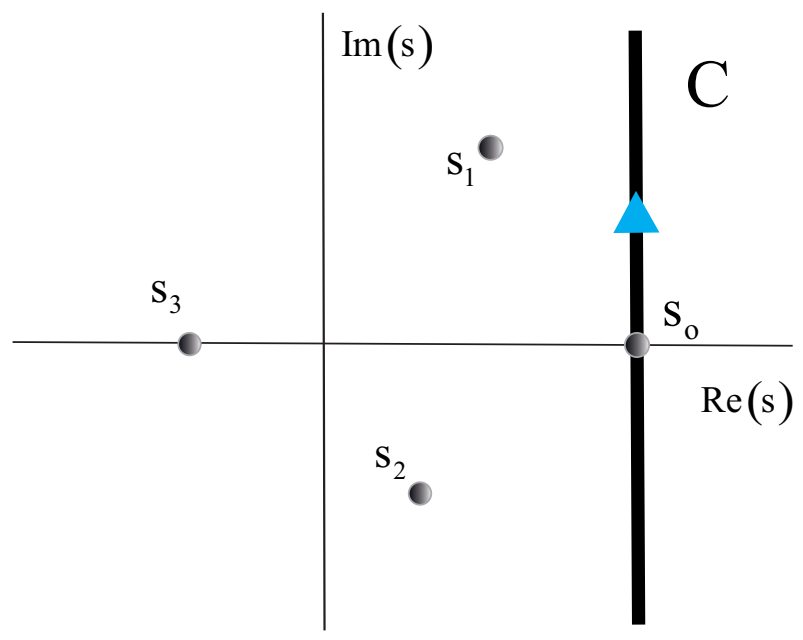

Figura 2.1: El contorno $C$ de la tranformada inversa de Laplace para el caso general donde la transformada tiene singularidades $s_{1}, s_{2}$ y $s_{3}$ en el semiplano izquierdo y el semiplano derecho de $\tilde{f}(s)$. El contorno tiene parte real $s_{o}$.

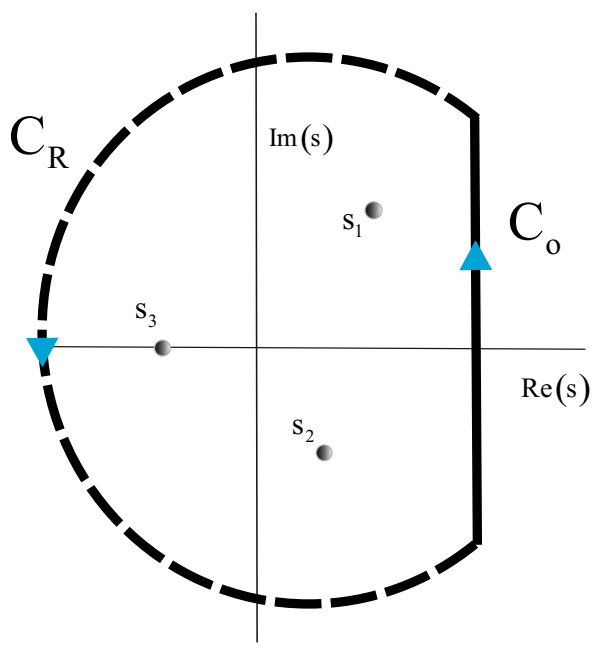

Figura 2.2: El contorno $C$ de la figura 2.1, que ahora denominaremos $C_{o}$ se cierra a la izquierda con una arco de circunferencia $C_{R}$. 


$$
\begin{aligned}
& p(x, y, z, t)=P+\varepsilon \tilde{p}(x, y, z, t)+c . c . \\
& \rho(x, y, z, t)=R+\varepsilon \tilde{\rho}(x, y, z, t)+c . c .
\end{aligned}
$$

donde $\varepsilon<<1$ y c.c. denotan los conjugados de las magnitudes complejas $\tilde{u}_{i}$, $\tilde{p}$ y $\tilde{\rho}$. Denotemos por $\tilde{u}, \tilde{v}$ y $\tilde{w}$ las componentes de la velocidad perturbada en las direcciones de los ejes $x, y, z$.

La localización de la superficie libre sometida a la acción de la tensión superficial, una vez perturbada, es descrita por $y_{i}+\xi_{i}(x, y, t)$, donde la función $\xi_{i}(x, y, t)$ con $\partial \xi_{i} / \partial t=v_{i}$ representa la perturbación de la superficie y el subíndice $i$ hace referencia a la misma.

A continuación procederemos a introducir transformadas de Fourier [115] con respecto a $x$ y $y$. La transformada de Fourier de $u(x, y, z, t)$ la podemos denotar como $u_{k}\left(k_{x}, k_{z}, y, t\right)=F_{x z}[u(x, y, z, t)]$ siendo

$$
F_{x z}[u(x, y, z, t)]=\frac{1}{2 \pi} \int_{-\infty}^{+\infty} d x \int_{-\infty}^{+\infty} \tilde{u}(x, y, z, t) \mathrm{e}^{i\left(k_{x} x+k_{z} z\right)} d z
$$

Este proceso lo podemos repetir para las restantes magnitudes perturbadas

$$
\begin{aligned}
u_{k}\left(k_{x}, k_{z}, y, t\right) & =F_{x z}[\tilde{u}(x, y, z, t)] \\
v_{k}\left(k_{x}, k_{z}, y, t\right) & =F_{x z}[\tilde{v}(x, y, z, t)] \\
w_{k}\left(k_{x}, k_{z}, y, t\right) & =F_{x z}[\tilde{w}(x, y, z, t)] \\
\rho_{k}\left(k_{x}, k_{z}, y, t\right) & =F_{x z}[\tilde{\rho}(x, y, z, t)] \\
p_{k}\left(k_{x}, k_{z}, y, t\right) & =F_{x z}[\tilde{p}(x, y, z, t)]
\end{aligned}
$$

y teniendo en cuenta la influencia de la tensión superficial [86], obtenemos

$$
\begin{gathered}
R \frac{\partial u_{k}}{\partial t}=-i k_{x} p_{k} \\
R \frac{\partial v_{k}}{\partial t}=-\frac{d p_{k}}{d y}-\rho_{k} g-k^{2} \sum_{i} \zeta_{i}^{(s)} T_{i}^{(s)} \delta\left(y-y_{i}\right) \\
R \frac{\partial w_{k}}{\partial t}=-i k_{z} p_{k}
\end{gathered}
$$




$$
\begin{gathered}
\frac{\partial \rho_{k}}{\partial t}=-v_{k} \frac{d R}{d y} \\
i k_{x} u_{k}+\frac{d v_{k}}{d y}+i k_{z} w_{k}=0
\end{gathered}
$$

Aquí, $\delta\left(y-y_{i}\right)$ denota la función delta de Dirac y $T_{i}^{(s)}$ es la tensión superficial de la superficie de separación $y=y_{i}$ entre los dos medios. Además, $k^{2}=$ $k_{x}^{2}+k_{z}^{2}$ es el cuadrado del número de onda.

Después de eliminar $p_{k}, \rho_{k}, u_{k} \mathrm{y} w_{k}$ de las ecuaciones anteriores, podemos obtener una ecuación para $v_{k}$

$$
k^{2}\left(R \frac{\partial^{2}}{\partial t^{2}}-g \frac{\partial R}{\partial y}\right) v_{k}=\frac{\partial}{\partial y}\left(\frac{\partial^{2}}{\partial t^{2}}\right)\left(R \frac{\partial v_{k}}{\partial y}\right)-k^{4} \sum_{i} v_{k i}^{(s)} T_{i}^{(s)} \delta\left(y-y_{i}\right)
$$

Ahora, trataremos el problema desde el punto de vista de un IVP e introduciremos la transformada de Laplace [115. Así que definiremos las transformada de Laplace en el plano complejo $s$ como

$$
v_{k s}(y, s)=\int_{0}^{\infty} v_{k}(y, t) e^{-s t} d t, \quad \operatorname{Re}(s)>0
$$

Aquí usaremos la propiedad de la transformada de Laplace de la derivada segunda

$$
\mathfrak{L}\left\{\frac{\partial^{2} v_{k}(y, t)}{\partial t^{2}}\right\}=s^{2} \mathfrak{L}\left\{v_{k}(y, t\}-s F_{1}-F_{2}=s^{2} \bar{v}_{k}(y, s)-s F_{1}-F_{2}\right.
$$

siendo $F_{1}$ y $F_{2}$ las condiciones iniciales que son impuestas en $t=0$

$$
F_{1}=\left.v_{k}(y, t)\right|_{t=0^{+}} ; F_{2}=\left.\frac{\partial v_{k}(y, t)}{\partial t}\right|_{t=0^{+}}
$$

La aplicación de la transformada de Laplace a 2.133 da lugar a

$$
k^{2}\left(s^{2} R-g \frac{d R}{d y}\right) v_{k s}-s^{2} \frac{d}{d y}\left(R \frac{d v_{k s}}{d y}\right)=H(y ; s, k)
$$

donde el término $H(y ; s, k)$ vendrá dado por

$$
H(y ; s, k)=\Gamma(y ; s, k)-k^{4} \sum_{i} \bar{v}_{k s, i}^{(s)} T_{i}^{(s)} \delta\left(y-y_{i}\right)
$$


siendo $\Gamma(y ; s, k)$ el término que engloba todas las condiciones iniciales

$$
\Gamma(y ; s, k)=k^{2} R\left[s F_{1}+F_{2}-\frac{1}{k^{2} R} \frac{d}{d y}\left(R s \frac{d F_{1}}{d y}\right)-\frac{1}{k^{2} R} \frac{d}{d y}\left(R \frac{d F_{2}}{d y}\right)\right]
$$

Habría que señalar que el primer miembro de la ecuación 2.137 es idéntico a la ecuación diferencial de la inestabilidad (2.98) obtenida mediante un análisis en modos normales (considerando nulas la viscosidad y tensión superficial) lo cual es lógico, ya que en un análisis modal no existiría el mencionado término $H(y ; s, k)$ que engloba las condiciones iniciales.

Podemos resolver esta ecuación diferencial (2.137) para $v_{k s}$ en el espacio de frecuencias con las apropiadas condiciones de contorno y obtener la relación de dispersión del problema. Por último, procederemos a invertir $v_{k s}(y, s)$ para obtener $v_{k s}(y, t)$ como función del tiempo. Así, la transformada inversa de Laplace de $v_{k s}(y, s)$ vendrá dada por

$$
v_{k}(y, t)=\frac{1}{2 \pi i} \int_{-i \infty+s_{o}}^{+i \infty+s_{o}} v_{k s}(y, s) e^{s t} d s
$$

siendo $s_{o}$ la parte real de un contorno de integración paralelo al eje imaginario ubicado a la izquierda de las singularidades de $v_{k s}(y, s)$. 

CAPÍTUlO 3

\section{Estudio mediante un fluido regularizado de una inestabilidad de Rayleigh-Taylor}

En este capítulo, llevaremos a cabo un cálculo de las tasas de crecimiento de la inestabilidad y las autofunciones para la inestabilidad de RayleighTaylor con viscosidad (VRTI). Analizaremos el problema desde el punto de vista de una geometría unidimensional 1D y bidimensional 2D. Los resultados obtenidos en una geometría 1D serán, en primer lugar, validados para el caso particular de fluidos no viscosos, con objeto de establecer una comparativa con los resultados previos obtenidos en la literatura. Asimismo, complementaremos el trabajo de $\mathrm{Yu}$ et al. [56] incorporando el efecto de la viscosidad. Esto nos permitirá comprobar cómo se ve modificado el modo principal, así como los secundarios, terciarios... en el caso de que la viscosidad esté presente.

\subsection{Plantemiento del problema}

En esta sección realizaremos un análisis de estabilidad lineal. Para estudiar la versión viscosa de la inestabilidad de Rayleigh-Taylor, asumiremos un flujo base de velocidad nulo y dos fluidos separados por una superficie libre. Analizaremos la evolución de la perturbación sobre el flujo base que hemos considerado. En particular, estamos interesados en el desarrollo de estructuras fluidas bidimensionales y en las tasas de crecimiento de la inestabilidad de diferentes modos inestables. Los cálculos los llevaremos a cabo tanto en dominios de computación unidimensionales (1D) como bidimensionales (2D), validando en ambos casos el caso no viscoso. Para la geometría en 1D, formularemos las ecuaciones como un sistema de tres, dos y una ecuación dependiendo del número de variables del fluido que eliminare- 
Capítulo 3. Estudio mediante un fluido regularizado de una 56 inestabilidad de Rayleigh-Taylor

mos por manipulaciones algebraicas. Dependiendo del número de ecuaciones utilizadas, el orden del sistema de ecuaciones diferenciales puede cambiar, consecuentemente, las condiciones de contorno deben ser reformuladas.

Denotaremos por $\rho_{1}, \nu_{1}, \mu_{1}$ y $\rho_{2}, \nu_{2}, \mu_{2}$ la densidad, la viscosidad cinemática, la viscosidad dinámica en la parte superior (fluido denso) y en la parte inferior (fluido ligero), respectivamente.

En este análisis, las magnitudes características de tiempo $t_{c}$, longitud $l_{c}$, velocidad $u_{c}$, presión $p_{c}$, densidad $\rho_{c}$, viscosidad dinámica $\mu_{c} \mathrm{y}$ viscosidad cinemática $\nu_{c}$ las definiremos en función de los siguientes parámetros

$$
\begin{aligned}
& t_{c}=\left(\nu_{m} / g^{2}\right)^{1 / 3}, l_{c}=\left(\nu_{m}^{2} / g\right)^{1 / 3}, u_{c}=\left(\mathrm{g} \nu_{m}\right)^{1 / 3}, p_{c}=\rho_{m} g\left(\nu_{m}^{2} / g\right)^{1 / 3} \\
& \rho_{c}=\rho_{m}, \mu_{c}=\mu_{m}, \nu_{c}=\nu_{m}
\end{aligned}
$$

siendo $\rho_{m}, \mu_{m}$ y $\nu_{m}$ los valores medios de la densidad, viscosidad dinámica y viscosidad cinemática, respectivamente, expresados como

$$
\rho_{m}=\frac{\rho_{1}+\rho_{2}}{2}, \mu_{m}=\frac{\mu_{1}+\mu_{2}}{2}, \nu_{m}=\frac{\nu_{1}+\nu_{2}}{2}
$$

y $g$ el módulo de la aceleración de la gravedad.

El fluido está gobernado por las ecuaciones incompresibles y newtonianas de Navier-Stokes en un determinado dominio $\Omega$.

Como flujo base, vamos a considerar dos fluidos superpuestos y estáticos $(\mathbf{U}=0)$ separados por una interfase difusa centrada en $y=0$ y sujeta a la aceleración de la gravedad en la dirección negativa del vertical $y$.

Por tanto, con objeto de describir la inestabilidad de Rayleigh-Taylor (RTI) en una interfase abrupta parece más razonable intentar resolver el problema con un solo flujo de densidad y viscosidad variables. Se establece, por tanto, una zona de transición o interfase difusa de espesor finito para luego tomar el límite de dicho espesor cuando éste tiende a cero y recuperar el caso de interfase abrupta. Para ello, tomaremos como perfiles de densidad $R$ y viscosidad dinámica $\mu$ los siguientes

$$
R=1+A_{\rho} \tanh \left(y / L_{s}\right) \quad \mu=1+A_{\mu} \tanh \left(y / L_{s}\right)
$$

donde $L_{s}$ es la longitud característica de los perfiles de densidad y viscosidad y $A_{\rho}, A_{\mu}$ los números de Atwood de la densidad y la viscosidad dinámica, respectivamente, dados por

$$
A_{\rho}=\frac{\rho_{1}-\rho_{2}}{\rho_{1}+\rho_{2}} \quad A_{\mu}=\frac{\mu_{1}-\mu_{2}}{\mu_{1}+\mu_{2}}
$$


Teniendo en cuenta las expresiones (3.3), podemos recuperar el caso de interfase abrupta estableciendo $L_{s} \rightarrow 0$.

A continuación, utilizaremos dos métodos numéricos diferentes, dependientes de la hipótesis utilizada para resolver las ecuaciones linealizadas de Navier-Stokes alrededor del flujo base. La primera formulación se basa en un método espectral de colocación de Chebyshev, cuando la geometría es simplificada a 1D, y el segundo se construye en una formulación variacional 2D de las ecuaciones, utilizando una discretización de segundo orden de elementos finitos basada en el método de Galerkin.

\subsubsection{Formulación 2D}

Estamos interesados en el análisis temporal de estabilidad sobre un dominio computacional 2D con condiciones de contorno o frontera homogéneas tipo Dirichlet en las fronteras superior e inferior del dominio, y condiciones periódicas en las fronteras derecha e izquierda. Consecuentemente, este marco no contiene cualquier coordenada $z$ de forma explícita. Una generalización tipo 3D de este marco, donde las perturbaciones asumen una longitud periódica de envergadura (i.e. dirección homogénea), definiéndose $L_{z}$ a través del número de onda real $k_{z}=\frac{2 \pi}{L_{z}} \in \mathbb{R}$, puede ser obtenida en [116].

El sistema resultante es analizado en un dominio computacional finito 2D $\Omega=[-L, L] \mathrm{x}[-H, H]$ en el plano $x y$. En este caso, una condición de frontera periódica es impuesta entre las fronteras situadas en $x= \pm L$, ver figura 3.1. Esta es una diferencia relevante si comparamos este análisis tipo 2D con el análisis clásico de la inestabilidad de Rayleigh-Taylor tipo 1D que asume solución periódica tanto en la dirección horizontal $x$ como en la de envergadura $z$ [56].

Por todo ello, el problema de partida se centra en la resolución del problema BiGlobal de autovalores dado por (2.46) sin asumir a priori la periodicidad en la dirección horizontal $x$ e imponiendo únicamente la condición $\hat{q}(-L, y)=\hat{q}(L, y)$ como parte del problema. La perturbación del flujo debe satisfacer las condiciones de frontera no deslizantes sobre las fronteras superior e inferior. Es decir

$$
\begin{aligned}
& \hat{u}(x, \pm H)=0 \\
& \hat{v}(x, \pm H)=0
\end{aligned}
$$

Para discretizar las ecuaciones, utilizamos una malla no estructurada de elementos triangulares. El problema de autovalores es resuelto mediante el 
Capítulo 3. Estudio mediante un fluido regularizado de una

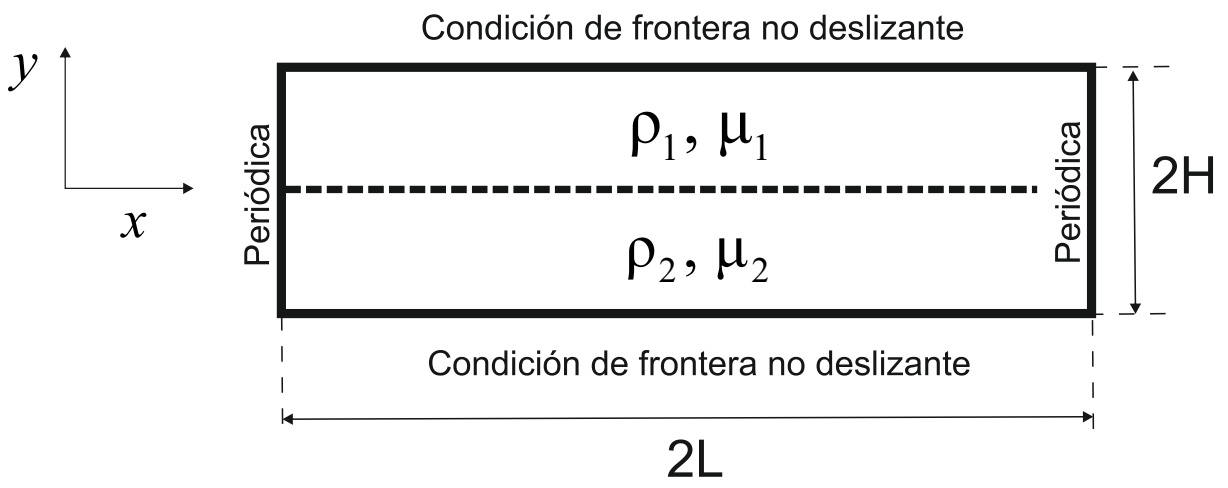

Figura 3.1: Representación de la geometría utilizada para una simulación 2D. La línea discontinua representa la coordenada $y=0$. Las condiciones de contorno son establecidas también en cada frontera.

método de Arnoldi usando una iteración del subespacio de Krylov [117, [110]. Con objeto de comprobar la precisión de los resultados durante el análisis de la estabilidad, el número de modos de la malla es incrementado hasta que los tres dígitos significativos del modo más inestable convergen.

\subsubsection{Formulación 1D}

Como hemos referido anteriormente, esta formulación 1D se llevará a cabo mediante un método espectral de colocación de Chebyshev. El dominio computacional $\Omega$ que vamos a utilizar sería $y \in[-H, H]$ con $N$ puntos discretos siendo las otras dos direcciones periódicas. En primer lugar, podríamos analizar el problema de autovalores desde el punto de vista de una formulación velocidad-presión-densidad que aparece planteada en (2.95), donde solamente impondremos condiciones de frontera homogéneas tipo Dirichlet, para la velocidad vertical y la derivada normal de la velocidad vertical de las perturbaciones, de la forma

$$
\hat{v}(y= \pm H)=0 \quad \mathrm{D} \hat{v}(y= \pm H)=0
$$

donde $D$ denota $d / d y$.

Por otro, el problema 1D también se podría estudiar desde un enfoque o formulación de velocidad vertical perturbada $\hat{v}$ tal y como queda reflejado 
en el problema de autovalores cuadrático (2.98).

La ecuación diferencial (2.98) en conjunción con las condiciones de contorno (3.7) constituyen el problema de autovalores cuadrático para $\gamma$. Habría que decir que 2.98 es la misma ecuación diferencial de cuarto orden analizada por [86] para dos fluidos de densidad y viscosidad cinemática constante, resuelta por Mikaelian [1], cuando las condiciones de salto son impuestas en la superficie libre compartida por ambas regiones fluidas.

\subsection{Resultados}

Para un valor fijo del número de onda $k$, se resuelve numéricamente el problema viscoso de autovalores dado por las expresiones (2.98) (en 1D) y 2.46) (en 2D) utilizando las respectivas condiciones de frontera (3.7) (1D) y (3.5)-(3.6) (2D), respectivamente, obteniéndose los autovalores y las correspondientes autofunciones. Un método espectral de colocación de puntos de Chebyshev y un método en elementos finitos (FEM) son utilizados para discretizar los dominios $1 \mathrm{D}$ y $2 \mathrm{D}$, respectivamente.

\subsubsection{Validación RTI sin viscosidad ni tensión superficial}

Como previa validación del problema 1D, estudiaremos el límite no viscoso $\mu=D \mu=0$ en ambas regiones en ausencia de tensión superficial $S=0$. El resultado analítico de la tasa de crecimiento adimensional de la inestabilidad RTI en una interfase abrupta es dada por la expresión [67]

$$
\gamma_{C}=\sigma=\sqrt{A_{\rho} k \tanh (k H)}
$$

Cuando la interfase no es abrupta, obtenemos un espectro completo cuando resolvemos numéricamente la versión no viscosa del problema de autovalores (2.98). Además del máximo autovalor, que ha sido ampliamente investigado y que se aproxima al valor clásico $\gamma_{C}$, también nos encontramos con otros muchos autovectores con autovalores más pequeños. Los modos RTI menos inestables no han sido lo suficientemente investigados hasta la fecha.

La figura 3.2 muestra la tasa de crecimiento lineal del modo superficial en función del número de onda normalizado para diferentes números de Atwood $A_{\rho} \in[0.2,0.8]$. Los resultados numéricos se han obtenido para un valor pequeño de la longitud característica del perfil de densidad $L_{s}=0.01$, caso que se corresponde con una interfase abrupta entre ambos fluidos. Los autovalores numéricos, para un número de puntos de Gauss-Lobatto $N=$ 
Capítulo 3. Estudio mediante un fluido regularizado de una

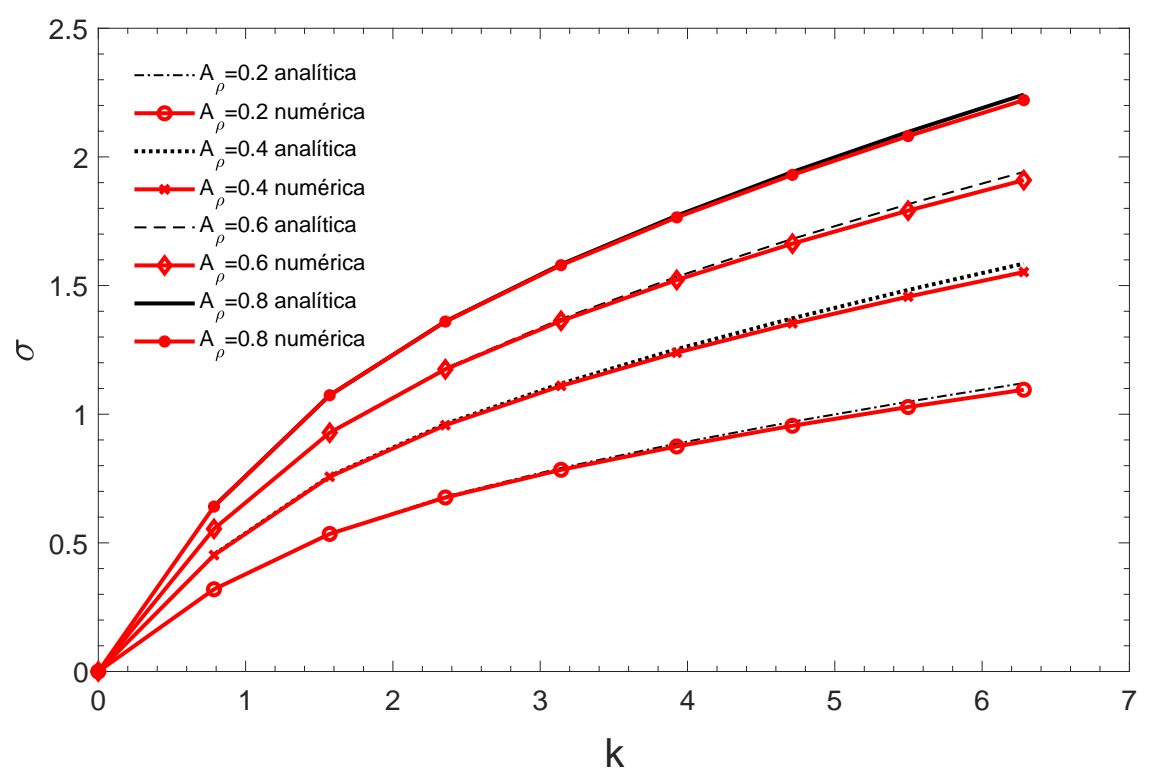

Figura 3.2: Tasa de crecimiento $\sigma$ de la inestabilidad RTI del autovalor más inestable, para el caso no viscoso, frente al número de onda $k$ para diferentes valores del número de Atwood $A_{\rho}=0.2,0.4,0.6,0.8$ para $H=1$ y $L_{s}=0.01$. La expresión analítica $(3.8)$ es añadida mediante líneas negras para establecer una comparativa.

256, son comparados con los resultados analíticos de (3.8), obteniéndose un buen acuerdo entre la solución analítica y la numérica.

Para una interfase de densidad no abrupta (transición suave entre regiones) la figura 3.3 muestra la tasa de crecimiento lineal de los cuatro modos mas inestables en función del número de onda normalizado $k$ para un número de Atwood $A_{\rho}=0.2$ y $L_{s}=0.203$. Nuestros resultados numéricos están en buen acuerdo con los $\gamma_{m}$ obtenidos mediante la expresión adimensional analítica aproximada (3.9) que es una generalización para dominios finitos de espesor $2 H$, y que hemos obtenido a partir de la aproximación proporcionada por $\mathrm{Yu}$ et al. [56] para $A_{\rho}<0.6 \mathrm{y}$ dominios infinitos.

$$
\gamma_{m}=\sqrt{\frac{A_{\rho}^{2} k^{2} \tanh (k H)}{\left(1+A_{\rho} k\right)\left[2(m-1)+A_{\rho} k\right]}} \quad m=1,2,3, \ldots
$$




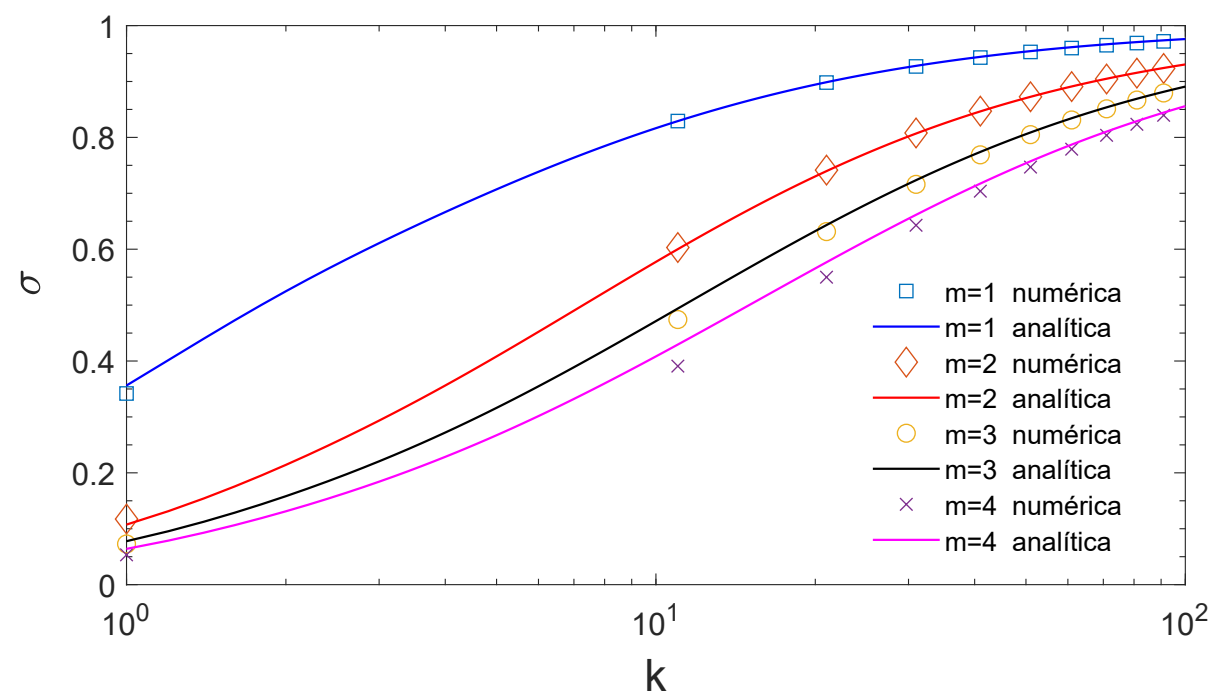

Figura 3.3: Tasa de crecimiento $\sigma$ del modo más inestable $(m=1)$ y de los tres siguientes modos de la RTI $(m=2,3,4)$ en función del número de onda $k$ para $A_{\rho}=0.2, L_{s}=0.203$ y $H=1$. Los resultados de la expresión analítica aproximada (3.9) han sido añadidos para establecer la oportuna comparación.

Como era de esperar para todos estos modos, la tasa de crecimiento de la inestabilidad lineal se incrementa con el número de onda, alcanzando la unidad cuando el número de onda tiende a infinito. El primer modo $(m=1)$ tiene la mayor tasa de crecimiento y su autovalor numérico está en buen acuerdo con el hallado mediante (3.9) el cual es representado en la figura 3.3 por una línea continua azul.

Las figuras 3.4 y 3.5 muestran las autofunciones de la velocidad vertical $\hat{v}$ correspondientes a los cuatro modos más inestables, para los números de onda $k=4$ y $k=6$, cuando el número de Atwood es $A_{\rho}=0.2$ siendo $L_{s}=0.1 \mathrm{y}$, por otro lado para $k=1 \mathrm{y} k=4$ siendo el número de Atwood $A_{\rho}=0.8$ y $L_{s}=0.203$. El primer caso representa dos fluidos de densidad constante con una interfase ligeramente difusa, y el segundo caso dos fluidos con una interfase difusa entre ambos. Como puede observarse, modos de orden superior presentan estructuras más complejas, especialmente en la interfase difusa de separación de los fluidos y en las proximidades de esta. Como ocurre en similares estudios, ver [56], el número de máximos y mínimos 
Capítulo 3. Estudio mediante un fluido regularizado de una inestabilidad de Rayleigh-Taylor

(a)
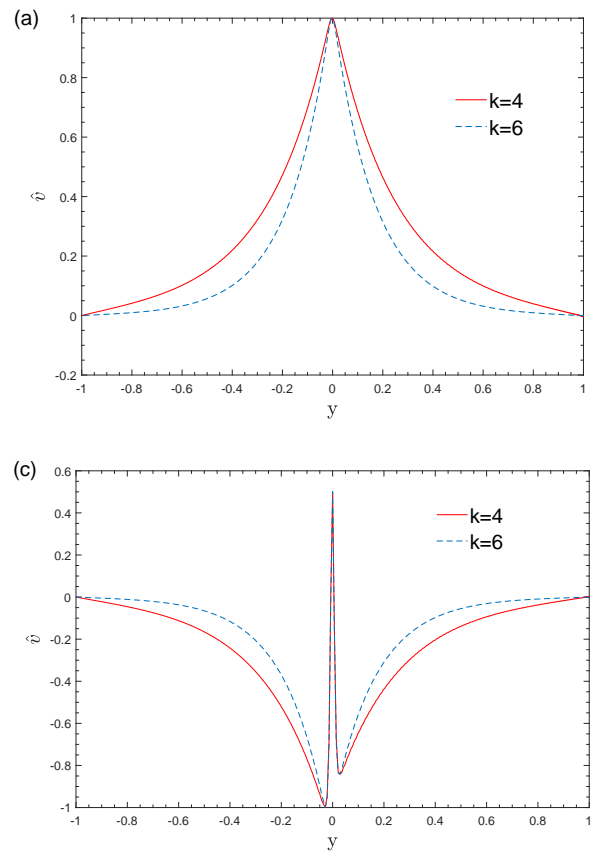

(b)

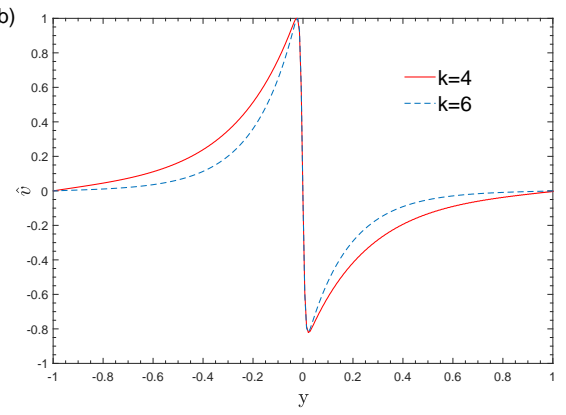

(d)

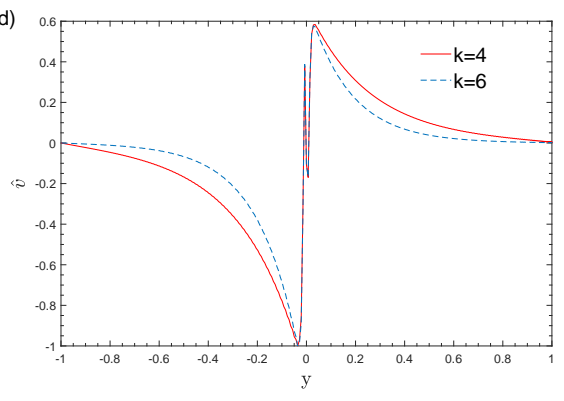

Figura 3.4: Autofunciones de la componente vertical de la velocidad $\hat{v}$ correspondientes a los cuatro modos más inestables $m=1$ (parte superior izquierda), $m=2$ (parte superior derecha), $m=3$ (parte inferior izquierda), $m=4$ (parte inferior derecha) para números de onda $k=4$ y $k=6$ y $A_{\rho}=0.2$. Otros parámetros son $L_{s}=0.1$ y $H=1$.

locales está relacionado con el orden de la autofunción. Esto significa que el primer modo contiene un máximo local, el segundo un máximo y un mínimo y el tercero dos mínimos y un máximo, etc... 
(a)

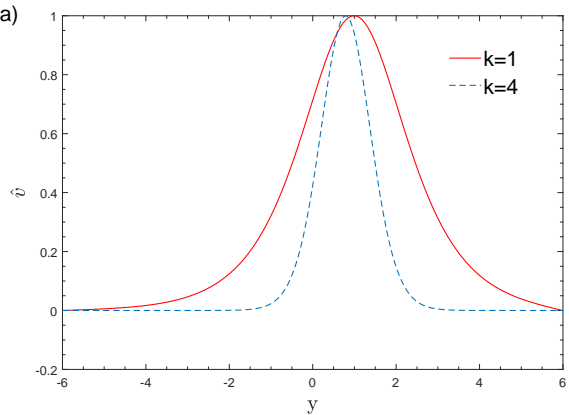

(c)

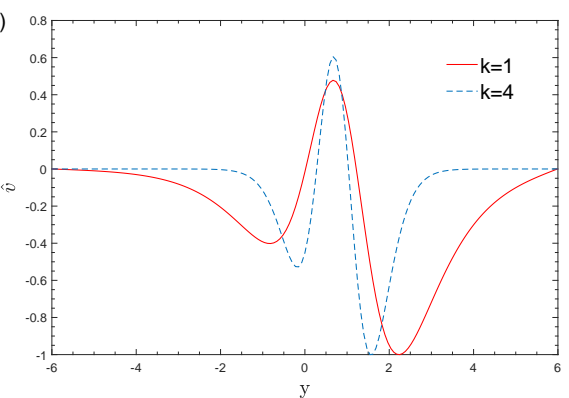

(b)

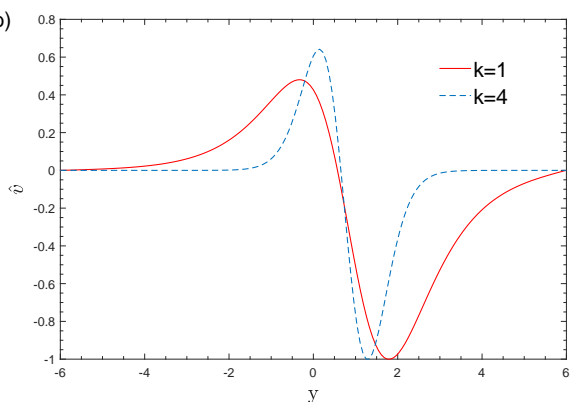

(d)

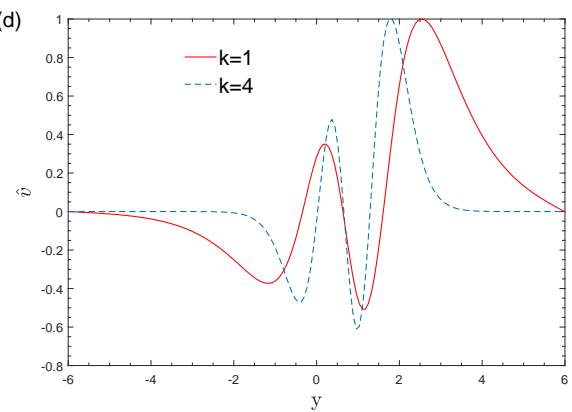

Figura 3.5: Autofunciones para la componente de la velocidad vertical $\hat{v}$ correspondientes a los cuatro modos más inestables $m=1$ (parte superior izquierda), $m=2$ (parte superior derecha), $m=3$ (parte inferior izquierda), $m=4$ (parte inferior derecha) para los números de onda $k=1 \mathrm{y} k=4 \mathrm{y}$ $A_{\rho}=0.8$. Otros parámetros son $L_{s}=0.203$ y $H=6$. 
Capítulo 3. Estudio mediante un fluido regularizado de una

\subsubsection{Problema viscoso sin tensión superficial}

\section{Formulación 1D}

La figura 3.6 muestra los resultados numéricos obtenidos cuando resolvemos el problema viscoso (2.98) utilizando una superficie libre de separación (interfase abrupta) sin tensión superficial, simulándola, para ello, con perfiles de viscosidad dinámica con una longitud característica $L_{s}=0.01$. Los resultados son comparados con los de Mikaelian [1] utilizando dos fluidos diferentes y condiciones de frontera en la interfase de separación entre ellos. Hemos realizado los cálculos para los números de Atwood de densidad y viscosidad $A_{\rho}=A_{\mu}=0.1,0.5,0.9$ utilizando dos dominios diferentes de computación de alturas $H=2$ y $H=4$. La comparación de nuestros resultados con los de Mikaelian están en muy buen acuerdo. Como era de esperar, difiriendo con respecto al caso no viscoso, en el caso viscoso las curvas no presentan una tendencia de crecimiento monótona, sino que presentan siempre un máximo valor.

La figura 3.7 muestra los dos modos más inestables correspondientes a las variables del fluido, como la velocidad vertical, densidad y presión, cuando el problema es resuelto para $k=1$ en $1 \mathrm{D}$ con $A_{\rho}=A_{\mu}=0.9$, longitud característica de los perfiles de densidad y viscosidad $L_{s}=0.5$ y tamaño computacional $H=1$. A diferencia de lo que se muestra comúnmente en la literatura, i.e. la única representación de las autofunciones de la velocidad vertical, nosotros representamos, además, las autofunciones de la densidad y la presión. La autofunción de la densidad sigue la tendencia de la autofunción de la velocidad vertical, coincidiendo en el número de máximos y mínimos que se corresponden con el orden de la autofunción. Sin embargo, la presión no sigue esta tendencia.

La figura 3.8 muestra la tasa de crecimiento de la inestabilidad para los cuatro modos más inestables siendo $A_{\rho}=A_{\mu}=0.9, L_{s}=0.6$ y $H=1$, cuando el cálculo es llevado a cabo en 1D. Las formas de las curvas siguen siendo para los modos $m=2,3,4$ semejantes a la obtenida para el modo más inestable $(m=1)$. En la figura 3.6 podemos observar que en todos los casos existe un máximo local.

\section{Formulación 2D}

Ahora el cálculo numérico será en $2 \mathrm{D}$, donde el dominio de computación es definido por un rectángulo de dimensiones $2 L$ y $2 H$ y la discretización es llevada a cabo por elementos finitos, utilizando elementos triangulares de tipo Taylor-Hood. La perturbación de la densidad fue aproximada por 
(a)

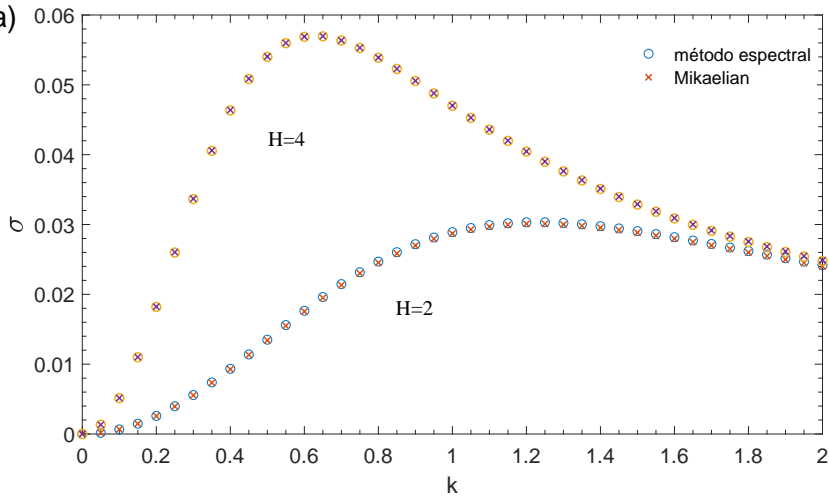

(b)

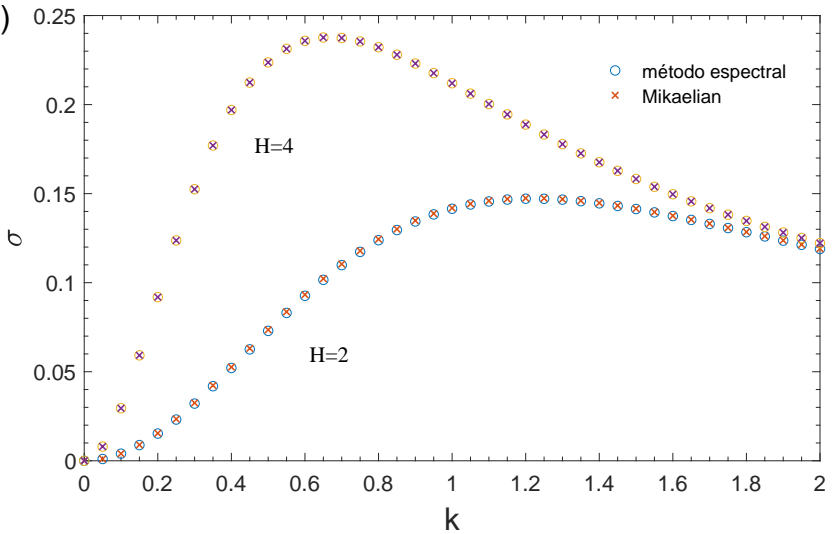

(c)

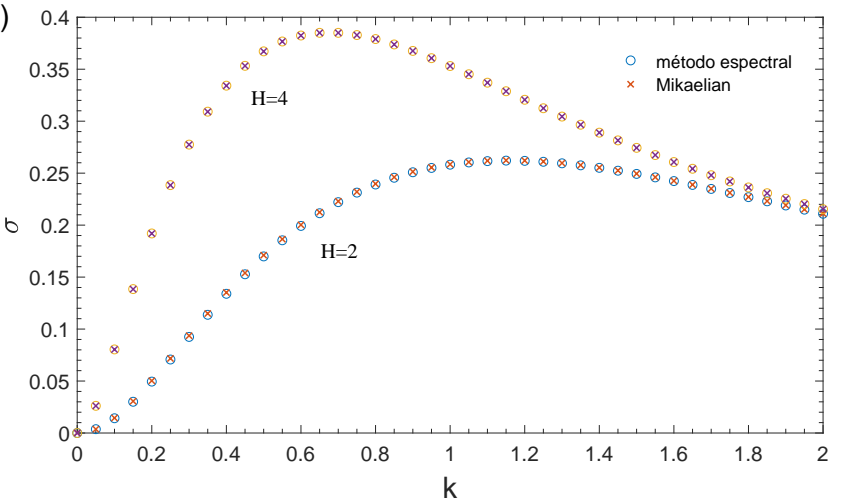

Figura 3.6: Dependencia de la tasa de crecimiento $\sigma$ con respecto al número de onda $k$ para $A_{\rho}=A_{\mu}=0.1$ (parte superior), $A_{\rho}=A_{\mu}=0.5$ (parte intermedia) y $A_{\rho}=A_{\mu}=0.9$ (parte inferior). Las curvas superior e inferior se corresponden con $H=4$ y $H=2$, respectivamente. Anteriores cálculos numéricos [1] y los obtenidos en el presente estudio mediante el método espectral $\left(L_{s}=0.01\right.$ y $\left.N=256\right)$ son representados. 
Capítulo 3. Estudio mediante un fluido regularizado de una
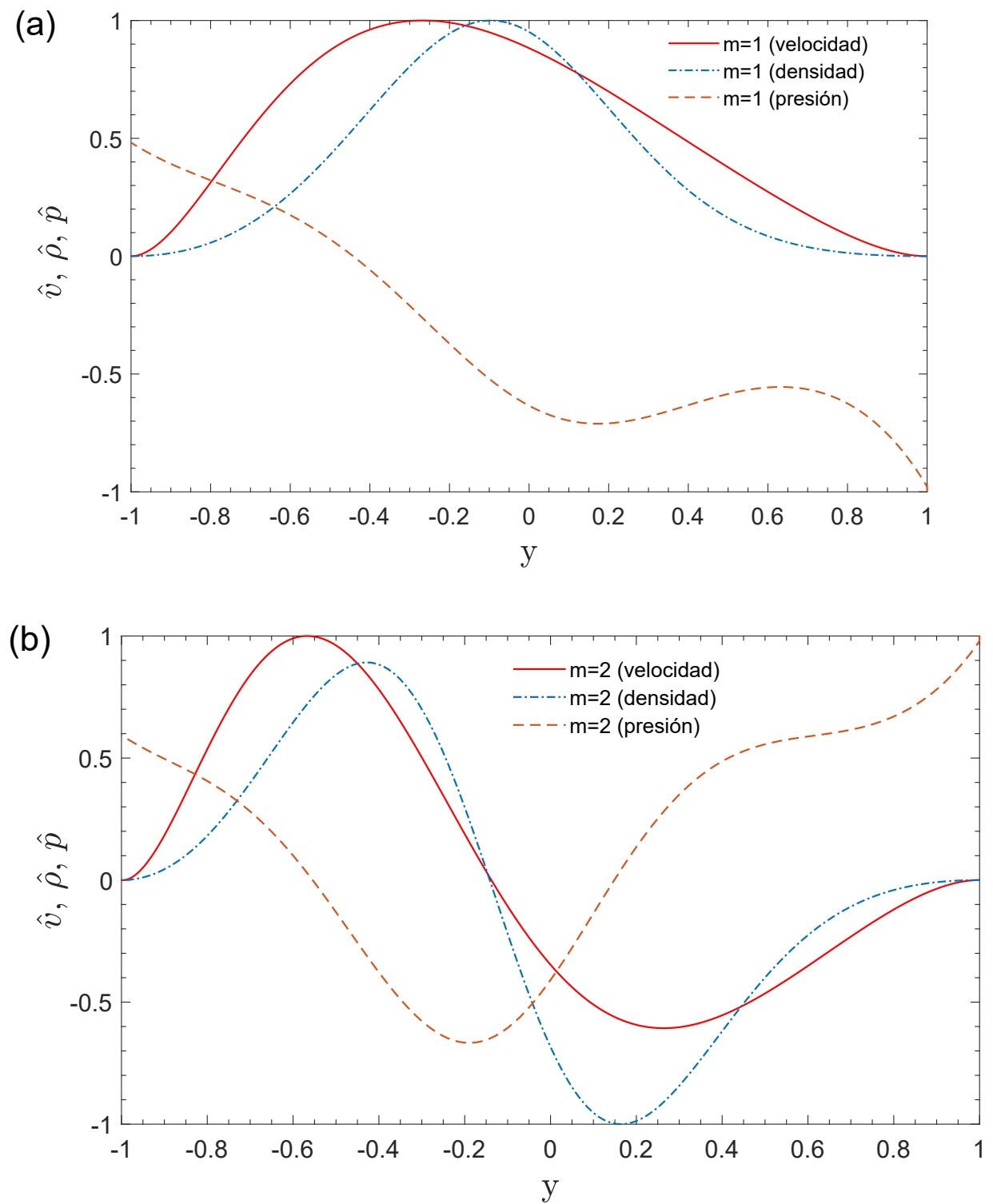

Figura 3.7: Autofunciones de los modos más inestables para la velocidad vertical, densidad y presión $m=1$ (arriba), $m=2$ (abajo) para el dominio $1 \mathrm{D}$ en el caso viscoso, donde $k=\pi, A_{\rho}=A_{\mu}=0.9, L_{s}=0.5$ y $H=1$. 


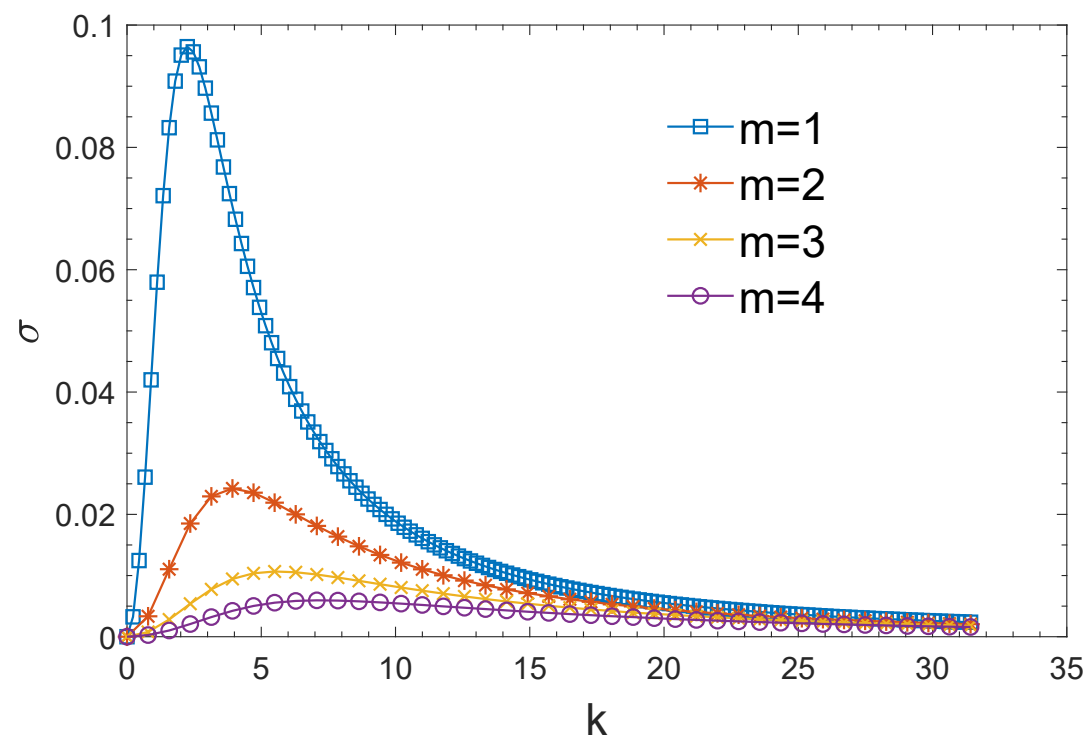

Figura 3.8: Dependencia de la tasa de crecimiento viscosa $\sigma$ con el número de onda $k$ siendo $A_{\rho}=A_{\mu}=0.9, L_{s}=0.5$ y $H=1$ para los cuatro modos más inestables $m=1,2,3,4$. 
Capítulo 3. Estudio mediante un fluido regularizado de una

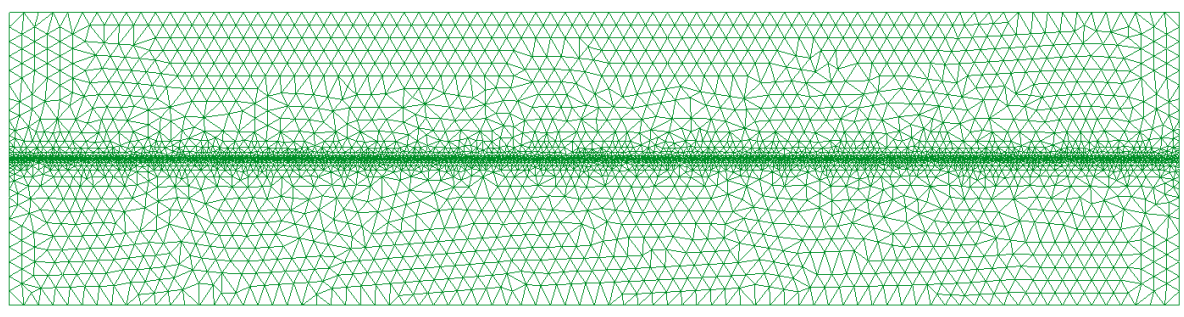

Figura 3.9: Malla utilizada para el cálculo viscoso en 2D.

polinomios de segundo orden. Todos los cálculos se han llevado a cabo en el dominio computacional $H=1$ y $L=4$.

El número de nodos cuadráticos de malla y elementos triangulares es 23145 y 11458 , respectivamente, donde la malla ha sido refinada alrededor de la línea horizontal $y=0$, ver figura 3.9 .

Es importante resaltar que la precisión numérica de este método de segundo orden es más baja, en este caso, que la obtenida por el método espectral 1D mediante polinomios de Chebyshev, pero permite cálculos más complejos en el futuro que no pueden ser llevados a cabo en $1 \mathrm{D}$, tales como flujos base confinados con paredes laterales a derecha e izquierda o en presencia de geometría compleja. En la figura 3.10, la dependencia de la tasa de crecimiento de la inestabilidad con el número de onda para el modo más inestable es calculada para varios cocientes de viscosidad y números de Atwood $A_{\rho}=A_{\mu}=0.8,0.6,0.4,0.2$ cuando la longitud característica de los gradientes de densidad y viscosidad es $L_{s}=0.01$. Al igual que en la formulación 1D, todas las curvas presentan un máximo local que decrece con el número de Atwood. Puede observarse que cuanto mayor sea el número de Atwood, mayor será la diferencia entre el valor calculado en $2 \mathrm{D}$ y el valor de referencia obtenido por métodos espectrales en el caso 1D. No obstante, la computación en 2D nos permite la posibilidad de estudiar geometrías y problemas más complejos que las estudiadas normalmente en la RTI.

En la figura 3.11, la autofunción de la perturbación más inestable, para todas las variables del fluido, es representada para grandes longitudes características del perfil de densidad $L_{s}=0.5$ y $k_{x}=\pi$. Se aprecia claramente la estructura periódica de las autofunciones con una longitud de onda definida $\lambda_{x}=2 \pi / k_{x}=2$.

En la figura 3.12 , la autofunción de la segunda perturbación más inestable es también representada para $H=1, A_{\mu}=A_{\rho}=0.9, L_{s}=0.5 \mathrm{y}$ 


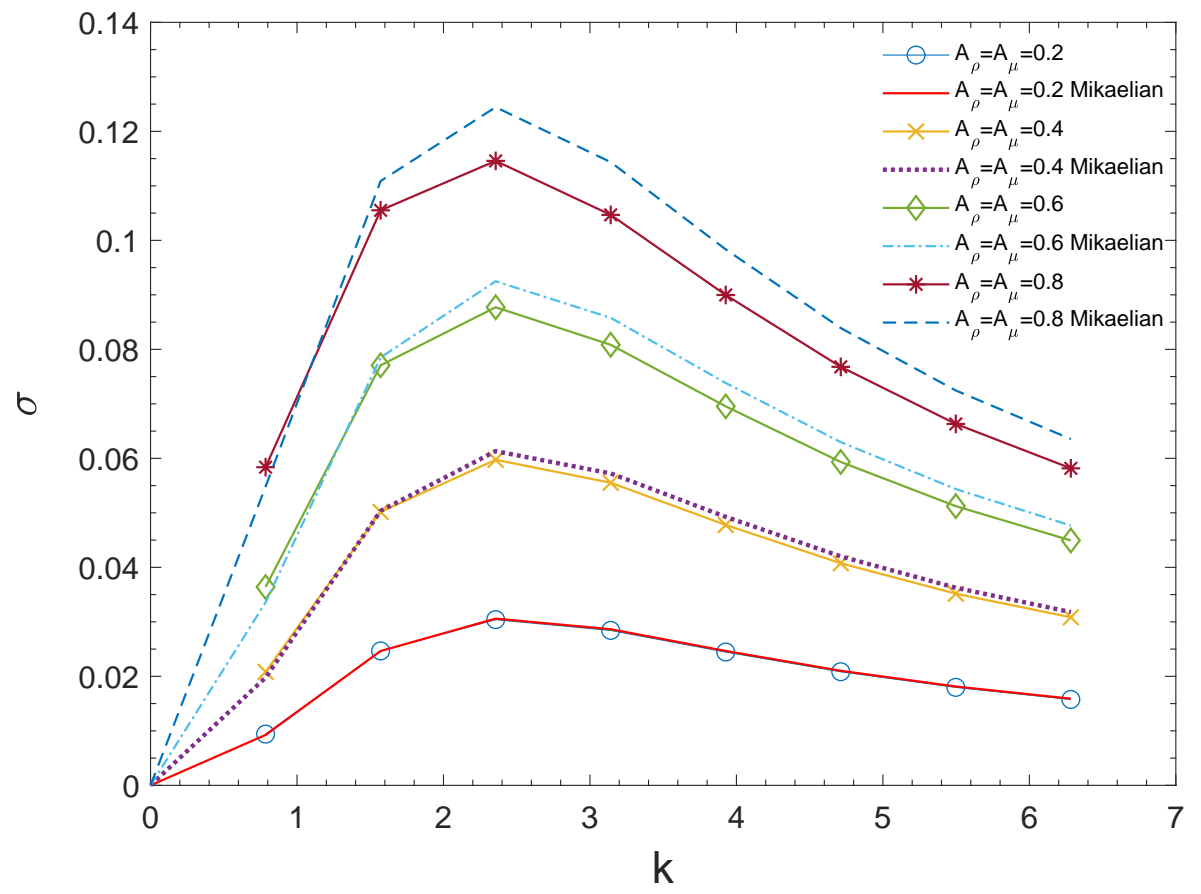

Figura 3.10: Dependencia de la tasa de crecimiento viscosa $\sigma$ con respecto al número de onda $k$ para $A_{\rho}=A_{\mu}=0.8,0.6,0.4,0.2$ y $H=1$. Los cálculos numéricos referenciados [1] y los obtenidos por FEM en 2D $\left(L_{s}=0.01\right)$ son representados. 
Capítulo 3. Estudio mediante un fluido regularizado de una inestabilidad de Rayleigh-Taylor
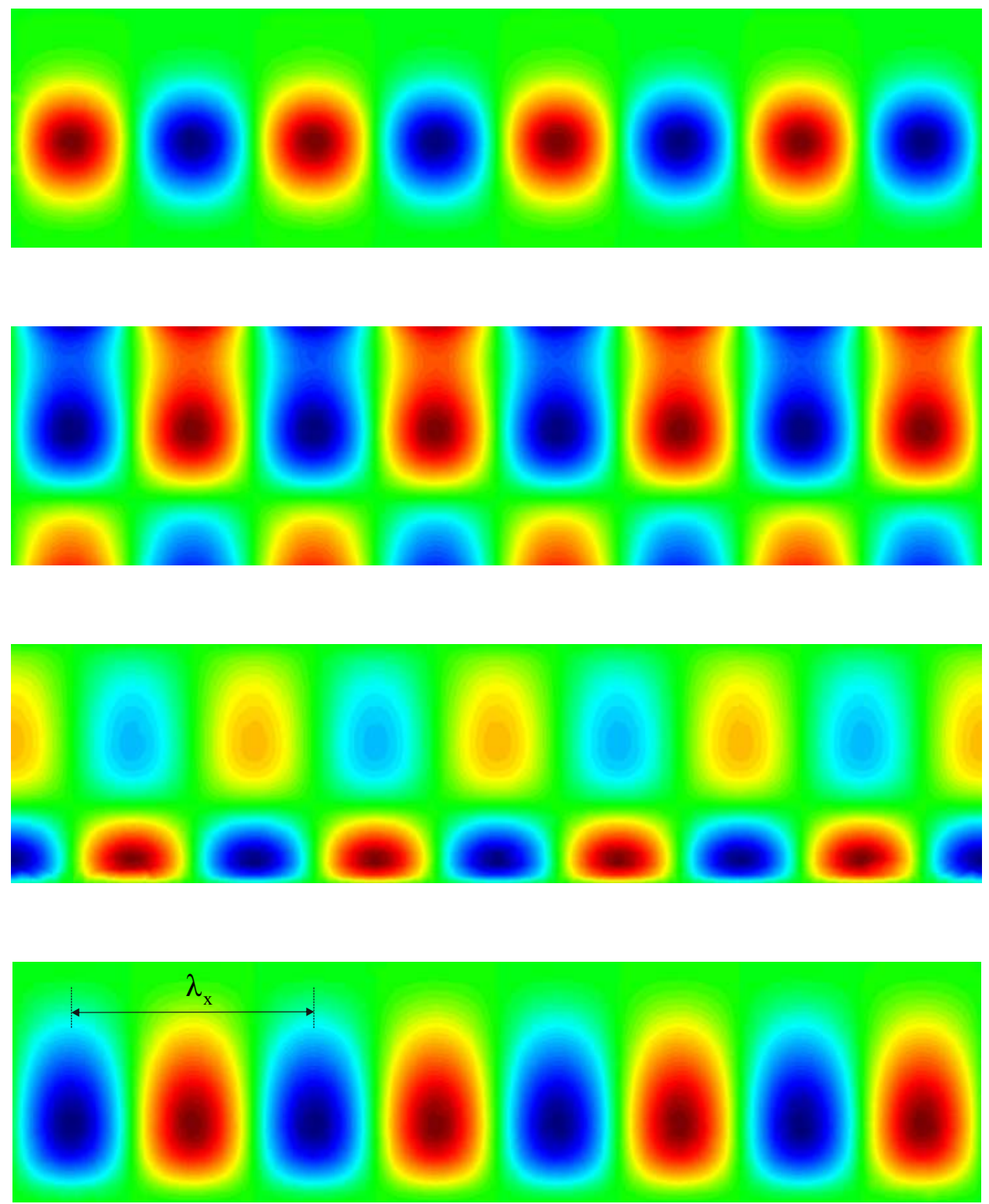

Figura 3.11: Estructura de la perturbación más inestable para el caso $k=\pi$, $H=1, L=4$ y $A_{\mu}=A_{\rho}=0.9$. Desde la gráfica superior a la inferior: densidad, presión, velocidad horizontal y velocidad vertical. Los cálculos fueron llevados a cabo con el parámetro de interfaz $L_{s}=0.5$. 
$k_{x}=\pi$. Como era de esperar, la estructura del segundo modo $(m=2)$ es más compleja, y el número de máximos y mínimos en las autofunciones de la velocidad vertical y densidad se incrementan con $m$. Asimismo, en la figura 3.13 se ha representado el tercer modo $(m=3)$ para el mismo valor de $k_{x}=\pi$ y los mismos valores de $A_{\mu}, A_{\rho}, L_{s}$ y $H$ anteriores.

\subsubsection{Problema viscoso con tensión superficial}

En la presente sección, llevaremos a cabo el análisis de estabilidad lineal de la RTI teniendo en consideración el efecto de la tensión superficial mediante el parámetro $S$. La justificación de este análisis está en que, hasta la fecha, no se ha llevado a cabo un estudio numérico completo (cálculo de autovalores y autofunciones) del problema de la RTI incluyendo simultáneamente los tres efectos: viscosidad, tensión superficial y espesor finito. Al respecto, habría que mencionar que Mikaelian [1] determinó una expresión analítica aproximada de la relación de dispersión que incluía simultáneamente los efectos de la tensión superficial, la viscosidad y el espesor finito. Por otro lado, este autor no representó las autofunciones del problema. La forma adimensional, con la normalización aquí utilizada, de dicha relación de dispersión aproximada viene dada por

$$
\sigma=-k^{2}+\sqrt{k^{4}-k\left(k^{2} S-A_{\rho}\right) \tanh (k H)}
$$

la cual utilizaremos en los siguientes apartados.

\section{Formulación 1D}

En primer lugar, validaremos los cálculos numéricos realizados en un dominio 1D mediante el método espectral anteriormente mencionado. Con este fin, vamos a comparar nuestros resultados con los que Reid [64] obtuvo para el caso de dos fluidos semi-infinitos. En la figura adjunta 3.14, se representan las tasas de crecimiento de la inestabilidad frente al número de onda para $L_{s}=0.01$ y diferentes valores del parámetro de tensión superficial $S$, y un espesor $H=10$ lo suficientemente grande para poder considerar los espesores de ambos fluidos como semi-infinitos. De la figura 3.14 se desprende un buen acuerdo entre los resultados obtenidos y los del referido autor [64].

En segundo lugar, sería de interés representar la relación de dispersión analítica aproximada (3.10) de Mikaelian [1] junto con la numérica obtenida en $1 \mathrm{D}$, para $L_{s}=0.01$ y diferentes valores de $S$ y espesor $H=10$, y llevar

a cabo la correspondiente comparación. En la figura 3.15 se puede observar 
Capítulo 3. Estudio mediante un fluido regularizado de una inestabilidad de Rayleigh-Taylor
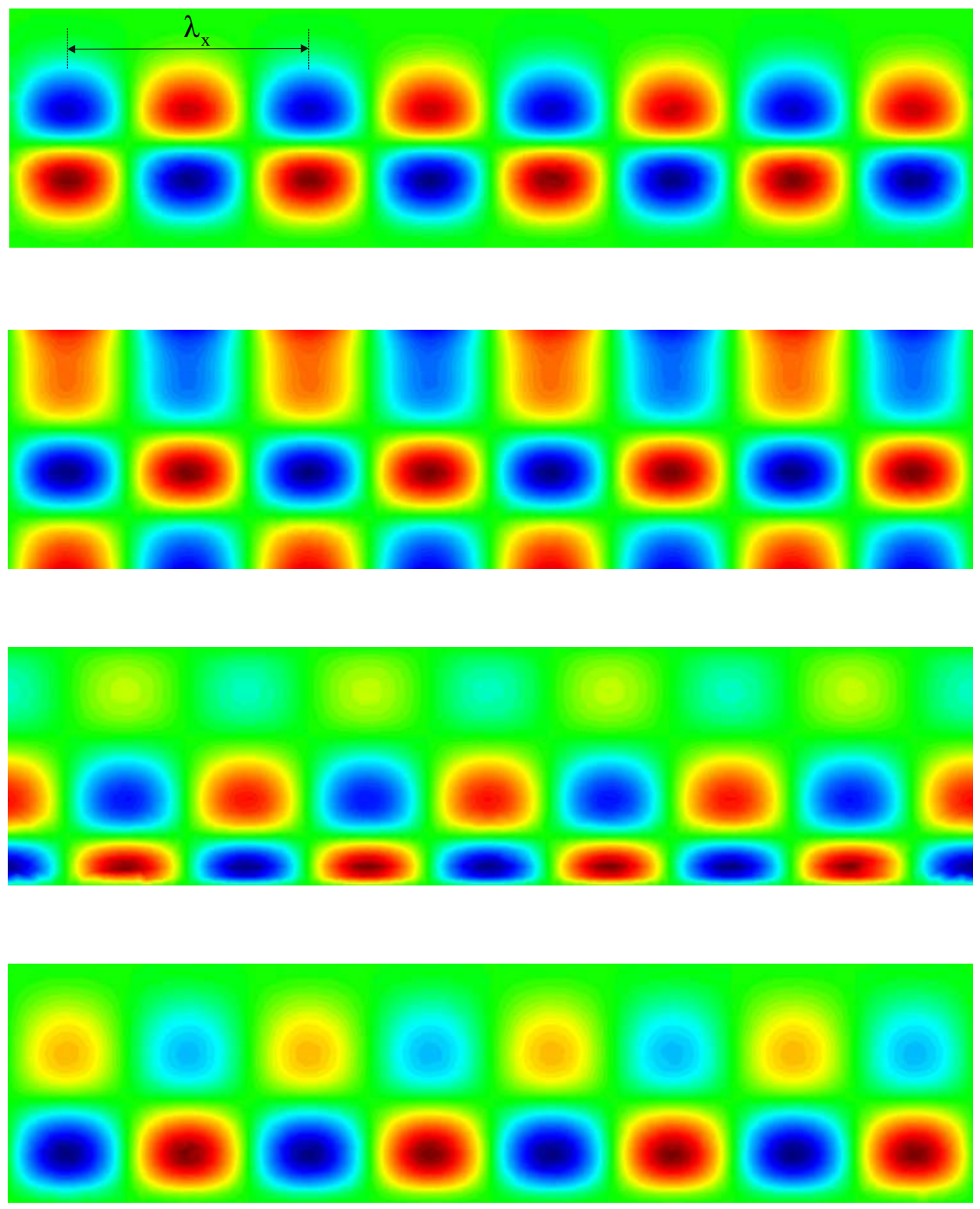

Figura 3.12: Estructura del segundo modo más inestable para el caso de $k=\pi, H=1$ y $A_{\mu}=A_{\rho}=0.9$. Desde la gráfica superior a la inferior: autofunciones de densidad, presión, velocidad horizontal y velocidad vertical. El cálculo se ha realizado para el parámetro de interfase $L_{s}=0.5$. 

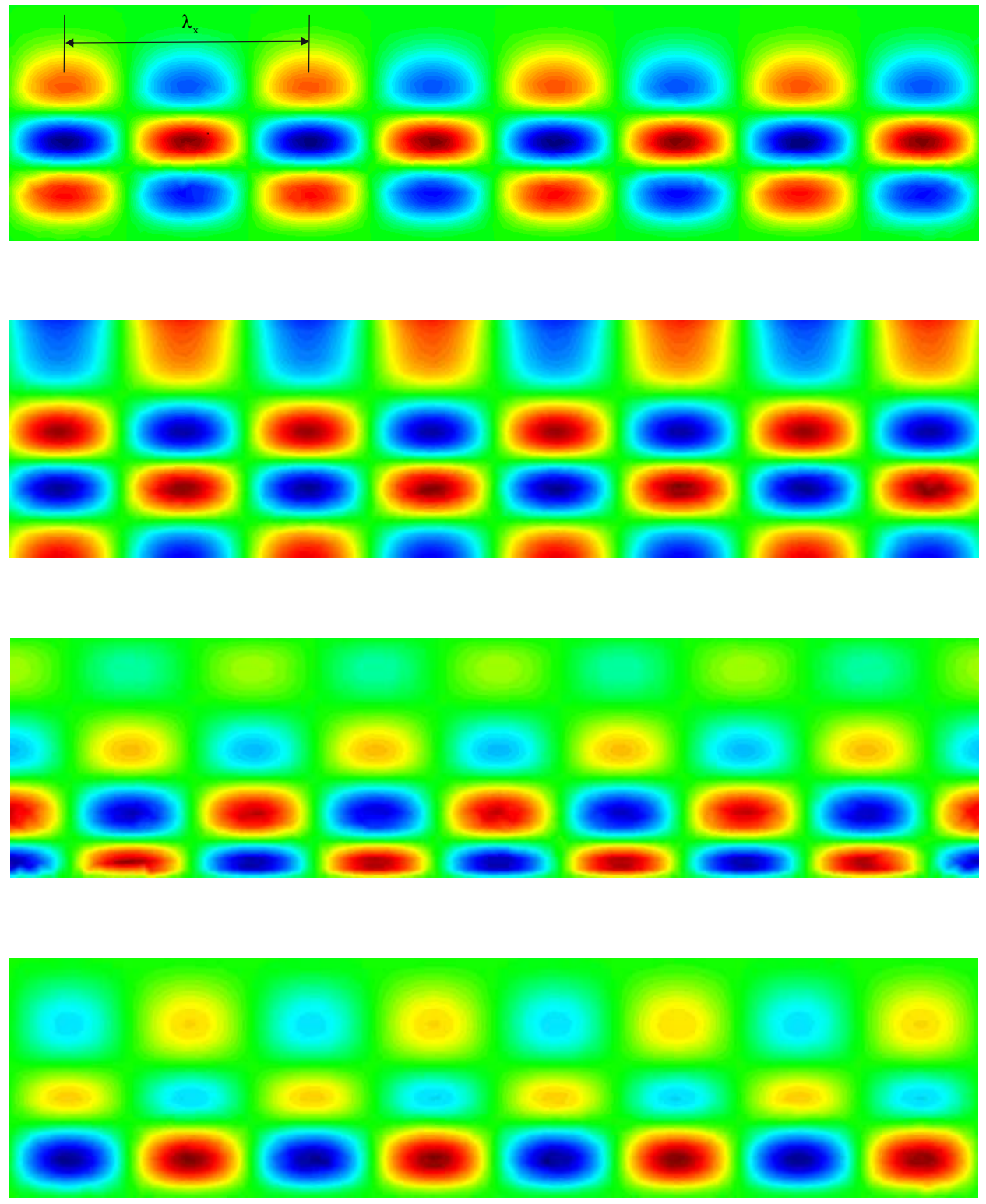

Figura 3.13: Estructura del tercer modo más inestable para el caso de $k=\pi$, $H=1$ y $A_{\mu}=A_{\rho}=0.9$. Desde la gráfica superior a la inferior: autofunciones de la densidad, presión, velocidad horizontal y velocidad vertical. El cálculo se ha realizado para el parámetro de interfase $L_{s}=0.5$. 
Capítulo 3. Estudio mediante un fluido regularizado de una inestabilidad de Rayleigh-Taylor

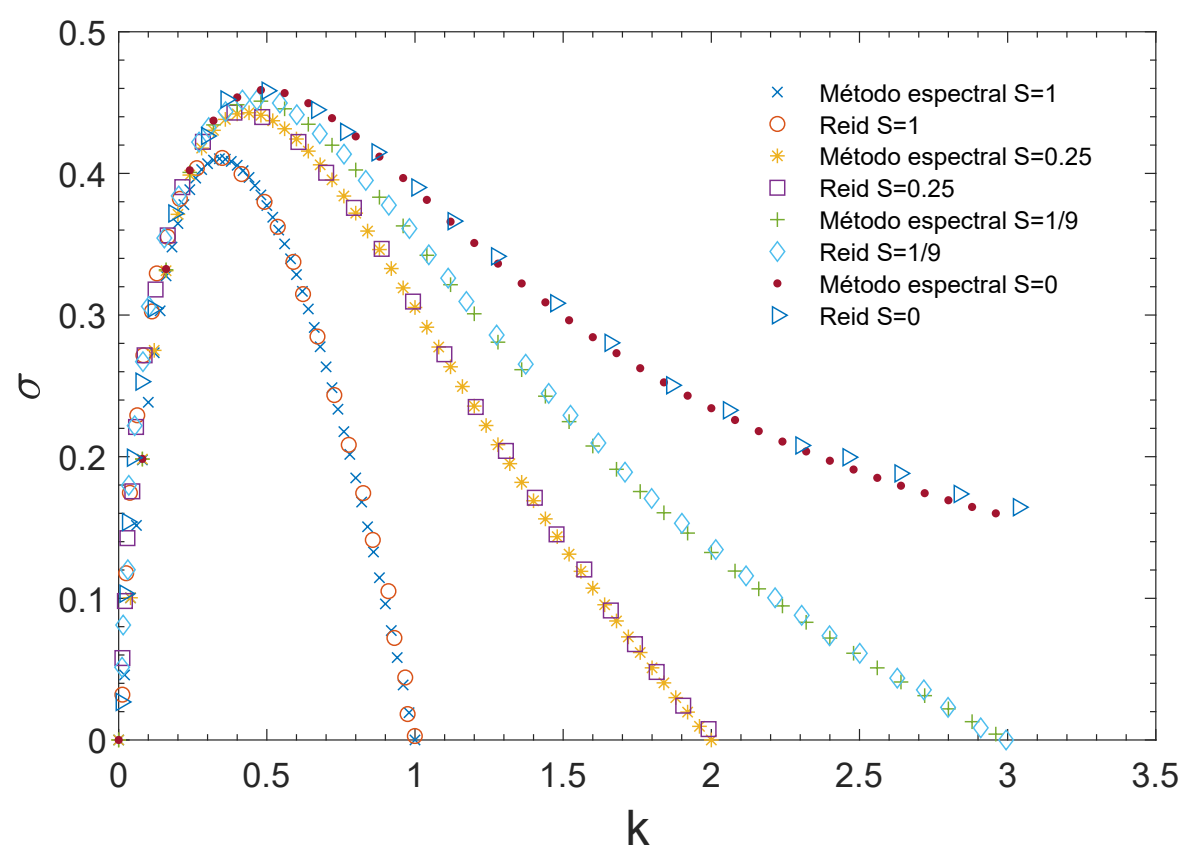

Figura 3.14: Tasa de crecimiento de la inestabilidad en función del número de onda para $S=0,1,0.25$ siendo $A_{\mu}=A_{\rho}=1.0, L_{s}=0.01$ y $H=10$.

que la expresión de Mikaelian [1] ofrece buenos resultados para números de onda grandes.

\section{Formulación 2D}

En este apartado, procederemos a abordar el efecto de la tensión superficial mediante el FEM (2D). Los resultados obtenidos se han validado con los correspondientes al método espectral (1D). En primer lugar, en la gráfica 3.16 se ha representado la tasa de crecimiento de la inestabilidad $\sigma$ en función del número de onda $k$ para $A_{\rho}=A_{\mu}=0.2, L_{s}=0.01, S=0.003$ y $H=1$ obtenida mediante los mencionados métodos. En segundo lugar, se ha llevado a cabo la misma representación, pero para $A_{\rho}=A_{\mu}=0.6$, $L_{s}=0.01, S=0.01$ y $H=1$ (ver 3.17). En ambas gráficas, también se han representado las relaciones de dispersión para el caso $S=0$, es decir, ausencia de tensión superficial, para establecer una comparación con los citados 


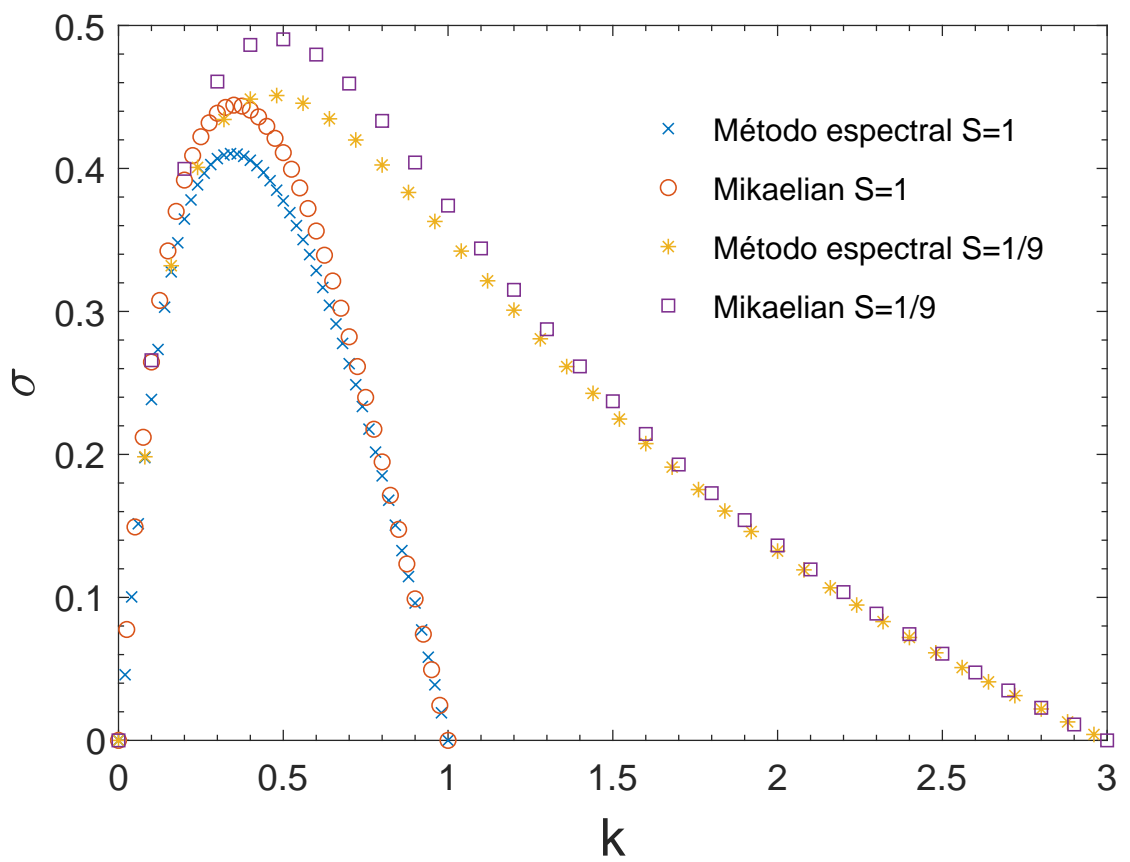

Figura 3.15: Tasa de crecimiento de la inestabilidad en función del número de onda para $S=1$ y $S=1 / 9$ siendo $A_{\mu}=A_{\rho}=1.0, L_{s}=0.01$ y $H=10$.

casos en los que la tensión superficial está presente. Como era de esperar, el efecto de la tensión superficial es el de estabilizar la RTI. En último lugar, en la figura 3.18 se muestran las autofunciones (densidad, presión, componentes horizontal y vertical de la velocidad perturbada) para el modo $k=\pi$, siendo $H=1, L=4, L_{s}=0.01, A_{\mu}=A_{\rho}=0.2$ y $S=0.003$. 
Capítulo 3. Estudio mediante un fluido regularizado de una inestabilidad de Rayleigh-Taylor

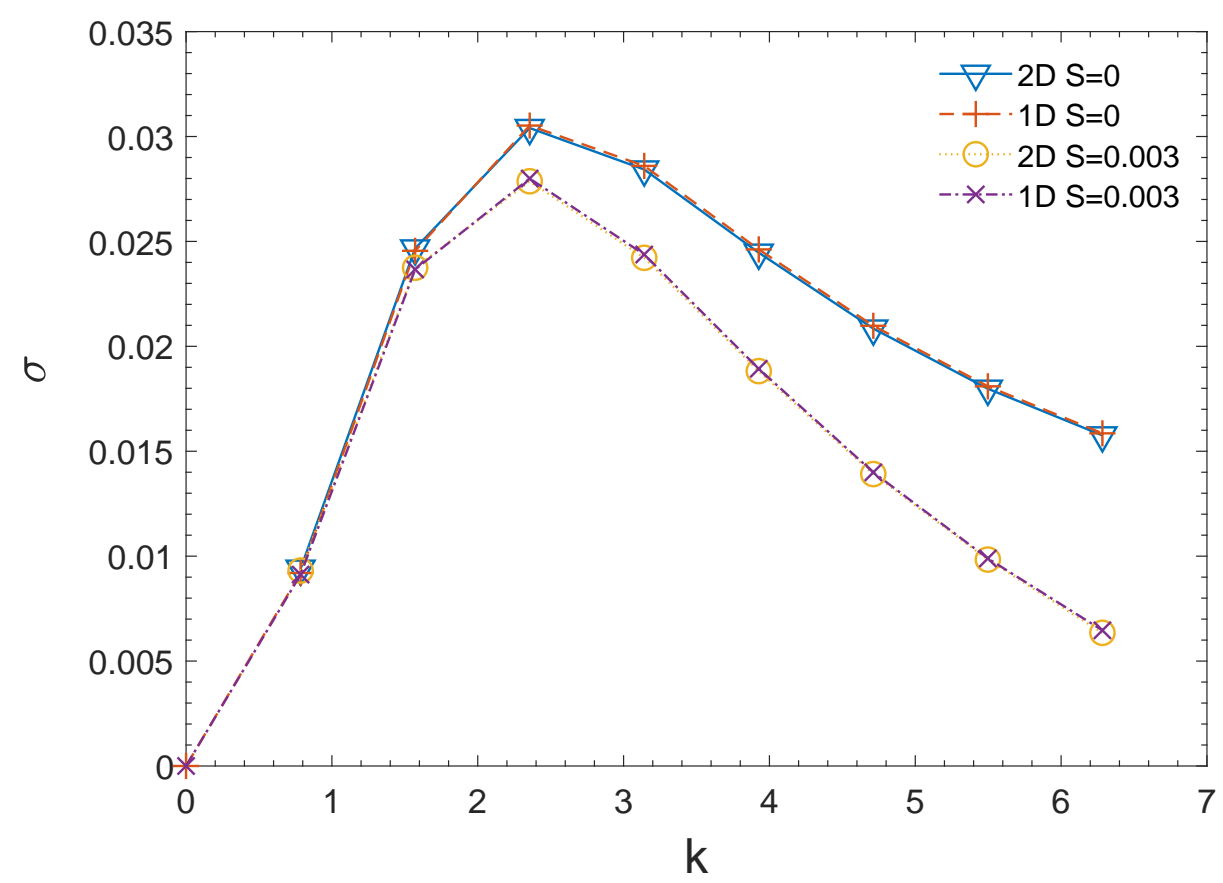

Figura 3.16: Tasa de crecimiento de la inestabilidad en función del número de onda, para $A_{\rho}=A_{\mu}=0.2, L_{s}=0.01, H=1, S=0$ y $S=0.003$.

\subsection{Conclusiones}

Es este capítulo, hemos llevado a cabo una descripción numérica completa de la RTI, tanto en el caso viscoso como en el no viscoso y en un simple dominio, donde las propiedades del fluido variaban según la función tangente hiperbólica, lo que nos ha permitido describir la RTI sin necesidad de implementar una condición de salto en la interfase de separación entre ambos fluidos. Hemos utilizado las formulaciones viscosa y no viscosa, tanto en el caso 1D como en el 2D. En la formulación no viscosa y para una interfase difusa entre los dos fluidos de espesor finito, hemos verificado que la RTI da lugar a una multiplicidad de autovalores y autofunciones, en consonancia con lo encontrado para fluidos semi-infinitos por $\mathrm{Yu}$ et al. [56]. Es de importancia resaltar que hemos estudiado el caso viscoso con interfase difusa de separación entre ambos fluidos de espesor finito, encontrando además de un modo viscoso principal, modos viscosos adicionales (secunda- 


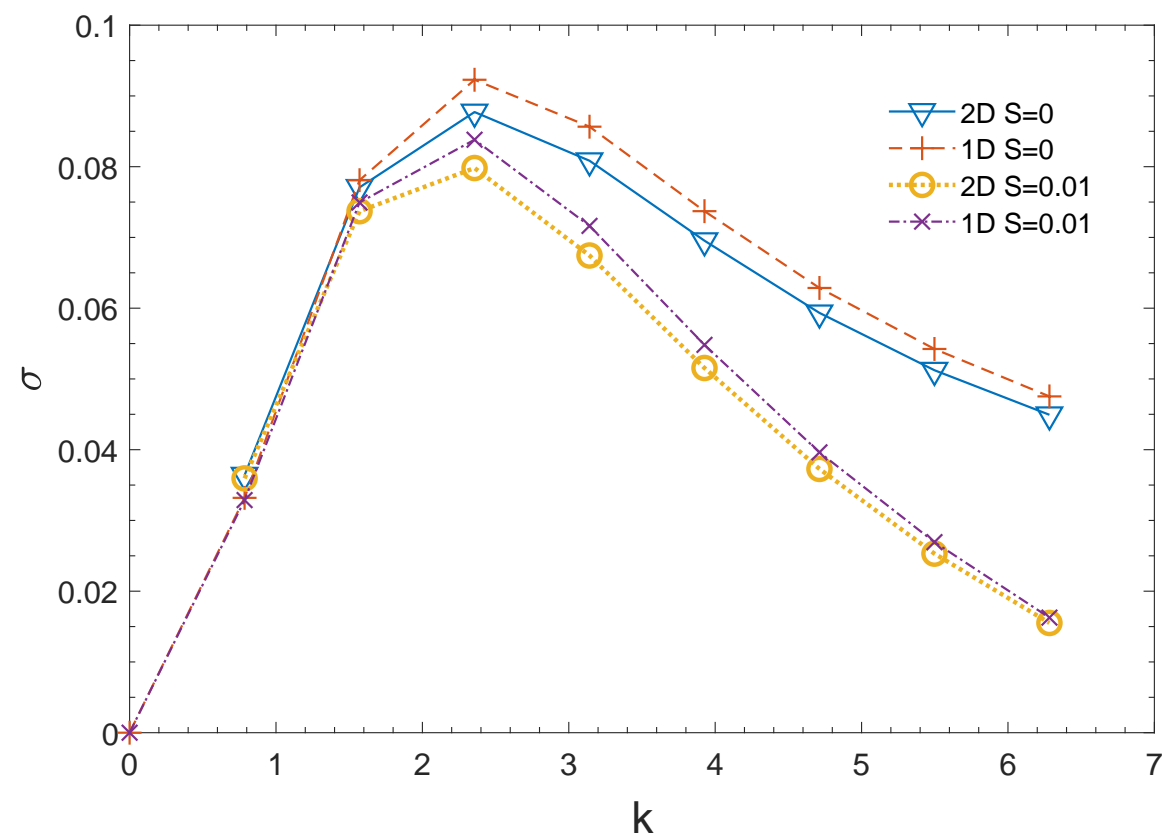

Figura 3.17: Tasa de crecimiento de la inestabilidad en función del número de onda, para $A_{\rho}=A_{\mu}=0.6, L_{s}=0.01, H=1, S=0$ y $S=0.01$.

rios, terciarios...) menos inestables que el principal cuya existencia, los de estos últimos, no se había puesto de manifiesto hasta la fecha, ni tan siquiera en el trabajo de Morgan et al. [59].

Hemos generalizado la expresión analítica aproximada de la tasa de crecimiento de la inestabilidad propuesta por Yu et al. [56] para el caso no viscoso y para un dominio finito, utilizándola para la correspondiente validación. Nuestra formulación numérica 1D, con un único fluido de densidad y viscosidad dinámica variables, tendiendo la longitud característica de la tangente hiperbólica a cero (interfase abrupta), ha sido comparada con el caso viscoso analizado por Mikaelian [1], obteniéndose buen acuerdo para un amplio rango de cocientes de densidad y viscosidad dinámica. En primer lugar, los resultados los hemos obtenido en un dominio computacional 1D utilizando discretización espectral. Posteriormente, hemos resuelto el problema en un dominio 2D con una discretización mediante FEM.

Asimismo, para el caso de una interfase abrupta con tensión superficial 
Capítulo 3. Estudio mediante un fluido regularizado de una inestabilidad de Rayleigh-Taylor
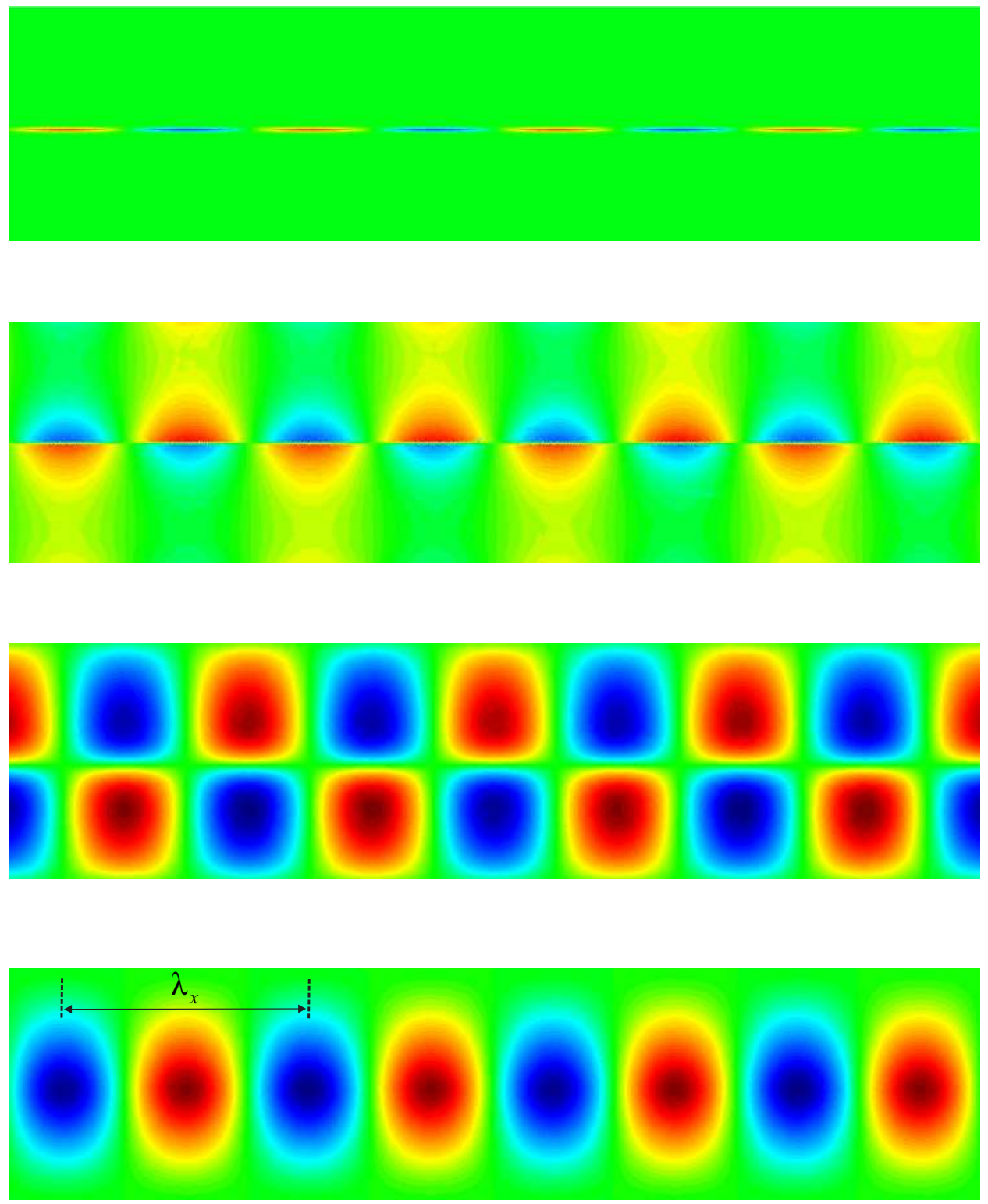

Figura 3.18: Perturbación para el caso $k=\pi, H=1, L=4$ y $A_{\mu}=A_{\rho}=0.2$ y $S=0.003$. Desde la gráfica superior a la inferior se presentan las amplitudes de las perturbaciones de la densidad, presión, velocidad horizontal y velocidad vertical. Los cálculos fueron llevados a cabo con el parámetro de interfase $L_{s}=0.01$. 
entre dos fluidos de espesor finito y viscosidad cinemática constante, se ha representado la relación de dispersión de la RTI, así como las autofunciones, complementándose de esta manera el trabajo de Mikaelian [1].

Las posibilidades dentro de la formulación 2D nos permiten incluir geometrías y condiciones de contorno más complejas, así como flujos base que no pueden utilizarse en el análisis clásico de la RTI. Además, la formulación que presentamos en este capítulo nos permite llevar a cabo un análisis de la estabilidad en presencia de objetos sumergidos o de soluciones bifásicas de las ecuaciones de Navier-Stokes previamente calculadas.

El presente trabajo también generaliza aplicaciones potenciales de mezclas de fluidos a aquellas donde la viscosidad tenga un papel relevante. Este capítulo cuantifica cómo la viscosidad afecta a modos de mayor orden los cuales tienen tasas de crecimiento de la inestabilidad más pequeñas que las del modo principal. En un futuro podríamos analizar la RTI dentro de recipientes confinados o de formas no rectangulares. 

Capítulo 4

\section{Inestabilidad de Kelvin-Helmholtz (KHI) en un medio heterogéneo}

En este capítulo, analizaremos desde el punto de vista numérico y lineal la inestabilidad de Kelvin-Helmholtz (KHI) en un medio heterogéneo. Para ello, regularizaremos los saltos en densidad y velocidad del flujo base mediante funciones del tipo tangente hiperbólica [94, 95]. De esta forma, podremos estudiar el comportamiento de la KHI tanto en transiciones abruptas como difusas del flujo base modificando dos parámetros básicos de control: los espesores de las capas de transición de los perfiles de densidad y velocidad. En nuestro estudio, y al igual que en los capítulos anteriores, emplearemos dos formulaciones: la primera unidimensional (1D) y la segunda bidimensional (2D). Haremos un estudio numérico del problema para un dominio finito obteniendo autovalores, autofunciones y líneas de corriente.

Ampliaremos el estudio de Gan et al. [94], obteniendo la relación de dispersión para un amplio rango de números de onda (mediante una simulación numérica estos autores solo analizaron el comportamiento del modo $k=10 \pi)$. Esto nos permitirá concluir si la validación de Gan et al. [94] con los resultados del flujo base utilizado por otros autores como Wang et al. [93], [101], Ye et al. [102] puede ser extrapolable a otro modos. Asimismo, completaremos el estudio con la representación de las autofunciones del problema a través de la formulación 2D.

Por último, nuestro análisis de estabilidad del flujo de tipo tangente hiperbólica para densidad y velocidad [94], 95] se extrapolará al caso en el que, además de la KHI, coexista RTI, estudio que fue realizado por Guzdar et al. [100] (utilizó un perfil en velocidad de tipo tangente hiperbólica y densidad de tipo gaussiana) y Ye et al. [102. 
Capítulo 4. Inestabilidad de Kelvin-Helmholtz (KHI) en un 82 medio heterogéneo

\subsection{Planteamiento del problema}

En este primer apartado llevaremos a cabo un análisis de estabilidad lineal. Con objeto de estudiar la versión clásica de la inestabilidad de KelvinHelmholtz, asumiremos un flujo base en velocidad no nulo y dos fluidos separados por una superficie libre, ver figura 1.3. Procederemos a analizar la evolución de la perturbación sobre el flujo base que hemos considerado. En particular, estamos interesados en el desarrollo de estructuras fluidas bidimensionales y en las tasas de crecimiento de la inestabilidad de diferentes modos inestables, así como sus frecuencias. Los cálculos los hemos llevado a cabo tanto en dominios de computación unidimensionales (1D) y bidimensionales (2D). Para el caso concreto de la geometría 1D, hemos formulado el correspondiente problema de autovalores generalizado planteado en (2.111).

El fluido estratificado no viscoso está gobernado por las ecuaciones incompresibles y newtonianas de Euler en un determinado dominio $\Omega$. Como flujo base, vamos a considerar dos fluidos superpuestos con diferente densidad y velocidad. Así pues, denotaremos por $\rho_{1}$ y $\rho_{2}$ las densidades en la parte superior (fluido denso) y en la parte inferior (fluido ligero), respectivamente, y $\mathbf{U}_{1}$ y $\mathbf{U}_{2}$ las velocidades de la fase densa y la ligera, respectivamente. Ambas fases estarán separadas por una interfase difusa centrada en $y=0 \mathrm{y}$ sujeta a la aceleración de la gravedad en la dirección vertical $y$.

En este análisis, las magnitudes características de tiempo $t_{c}$, longitud $l_{c}$, velocidad $u_{c}$, presión $p_{c}$ y densidad $\rho_{c}$ las definiremos en función de los siguientes parámetros

$$
\begin{gathered}
l_{c}=\left(U_{1}-U_{2}\right)^{2} / g, t_{c}=\left(U_{1}-U_{2}\right) / g, u_{c}=U_{1}-U_{2}, \\
p_{c}=\rho_{c} g l_{c}, \rho_{c}=\rho_{m}
\end{gathered}
$$

siendo $g$ el módulo de la aceleración de la gravedad y $\rho_{m}$ la densidad media expresada como

$$
\rho_{m}=\frac{\rho_{1}+\rho_{2}}{2}
$$

Para la formulación unidimensional (1D), este dominio $\Omega$ vendrá dado por el intervalo $\Omega=[-H, H]$ siendo $H$ el espesor de cada fase. Por otro lado, para la formulación bidimensional (2D) el dominio será un rectángulo de altura $2 H$ y anchura $2 L$ contenido en el plano XY, matemáticamente expresado como $\Omega=[-L, L] \mathrm{x}[-H, H]$.

Por tanto, con objeto de describir numéricamente la inestabilidad de Kelvin-Helmholtz en una interfase abrupta parece más razonable intentar 
resolver el problema asumiendo una zona de transición de espesor finito, tanto para la densidad como la velocidad del flujo base, para luego tomar el límite del espesor cuando éste tiende a cero. Para ello, podemos tomar como flujo base los perfiles adimensionales de densidad $R$ y velocidad $U$, que fueron utilizados por Faganello et al. [95] y Gan et al. 994 que en nuestra adimensionalización podríamos expresar como

$$
R=1+A_{\rho} \tanh \left(y / L_{\rho}\right) \quad U=U_{o} \tanh \left(y / L_{u}\right)
$$

donde $U_{o}$ es una constante, $2 L_{\rho}$ y $2 L_{u}$ representan los espesores de las zonas de transición de densidad y velocidad, respectivamente, y $A_{\rho}$ el número de Atwood de la densidad dado por

$$
A_{\rho}=\frac{\rho_{1}-\rho_{2}}{\rho_{1}+\rho_{2}}
$$

Como se ha mencionado anteriormente, otro flujo base con un comportamiento semejante al anterior sería el utilizado por Wang et al. [93, [101, Ye et al. [102. La densidad adimensional de este flujo, teniendo en cuenta (4.1), se podría expresar como

$$
R(y)=\left\{\begin{array}{cc}
1+A_{\rho}-A_{\rho} e^{-y / L_{\rho}} & y>0 \\
1-A_{\rho}+A_{\rho} e^{y / L_{\rho}} & y<0
\end{array}\right.
$$

siendo la velocidad adimensional

$$
U(y)= \begin{cases}U_{o}-U_{o} e^{-y / L_{u}} & y>0 \\ -U_{o}+U_{o} e^{y / L_{u}} & y<0\end{cases}
$$

A modo de ilustración, en la figura 4.1 se ha representado el flujo base dado por 4.3 para $A_{\rho}=0.6, H=1 \mathrm{y} U_{o}=1$. Como se puede observar en la figura 4.1 (gráfica derecha), podemos recuperar el caso clásico de KHI con transición abrupta de flujo base estableciendo $L_{\rho} \rightarrow 0$ y $L_{u} \rightarrow 0$.

Por otro lado, en la gráfica 4.2 hemos representado el flujo base (4.3) frente al definido por (4.5) y (4.6) para $A_{\rho}=0.6, L_{\rho}=L_{u}=0.1, U_{o}=1$. Se observa una cierta similitud entre ambos flujos base para los mencionados parámetros.

No obstante, es de interés comparar las distribuciones de vorticidad $D U(y)$ de ambos flujos. Por ello, en la gráfica 4.3 se ha representado la distribución de vorticidad para ambos perfiles de velocidad 4.3 y (4.6) siendo $U_{o}=1$ y $L_{u}=0.1$.

Como se observa en la figura 4.3 , la distribución de vorticidad del flujo base (4.3) tiene dos puntos de inflexión, de los que carece el flujo base de 

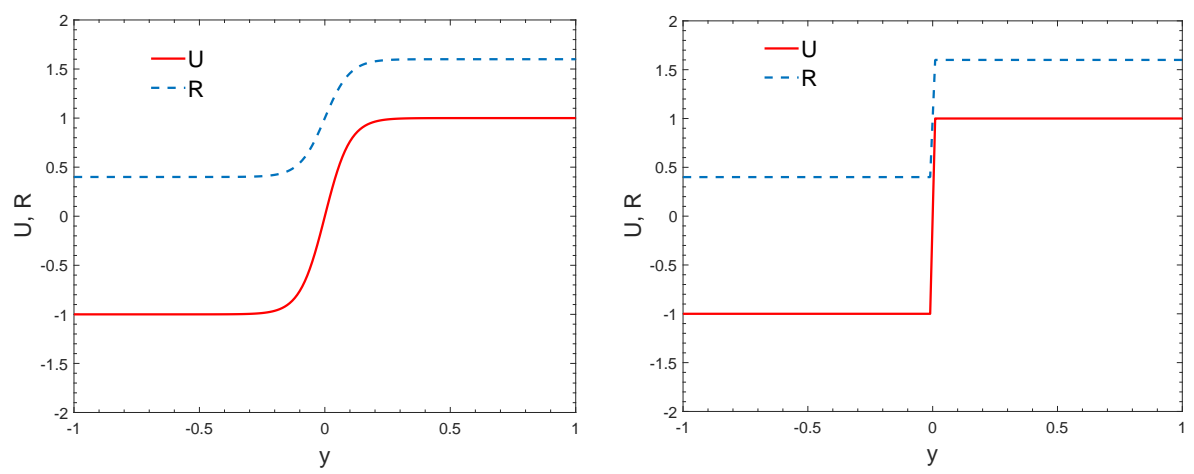

Figura 4.1: Representación del flujo base en velocidad y en densidad dado por la expresión 4.3 para $H=1, A_{\rho}=0.6$ y $U_{o}=1$. En la gráfica izquierda el flujo base está caracterizado por $L_{\rho}=L_{u}=0.1$ (transición difusa) y en la derecha, $L_{\rho}=L_{\rho}=10^{-3}$ (transición abrupta).
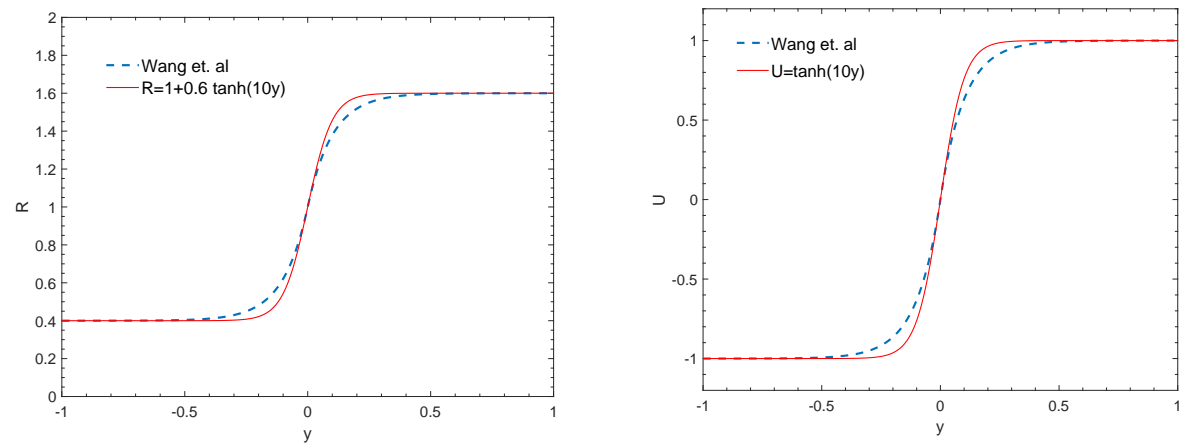

Figura 4.2: Comparativa entre flujos base en densidad (izquierda) y velocidad (derecha) para $U_{o}=1, H=1, A_{\rho}=0.6$ y $L_{\rho}=L_{u}=0.1$. En línea discontinua se ha representado el flujo base dado por 4.5 y 4.6. En línea continua, el flujo base definido por (4.3). 

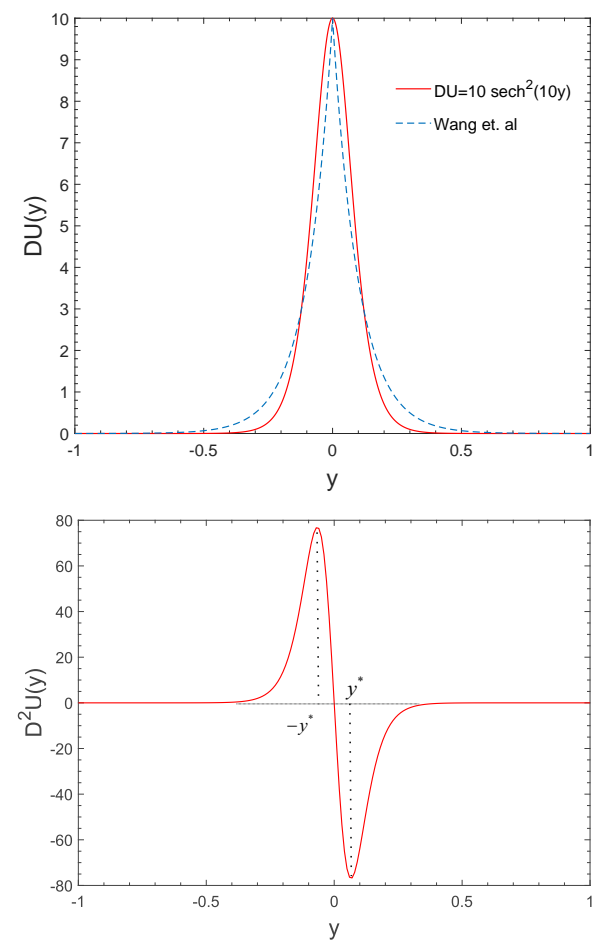

Figura 4.3: Distribución de vorticidad (gráfica superior) y gradiente de vorticidad (gráfica inferior) del flujo base dado por (4.3) para $U_{o}=1, H=1$, $L_{u}=0.1$. En línea discontinua se ha representado la distribución de vorticidad correspondiente al flujo base dado por 4.6. En línea continua, la vorticidad del flujo base de 4.3 . 
Wang et al. 93]. Estos puntos de inflexión se corresponden con el máximo y el mínimo $y^{*}= \pm 0.065$ de la distribución del gradiente de vorticidad (ver figura 4.3). Desde el punto de vista de la teoría interacción de ondas (WIT), Carpenter et al. [44] considera a estos puntos $y^{*}= \pm 0.065$ las posiciones centrales de dos interfases difusas de vorticidad con signo opuesto que interaccionan entre sí, a través de ondas de vorticidad, de forma semejante a la interacción entre dos interfases abruptas de vorticidad. Como resultado de esta interacción, la tasa de crecimiento de la inestabilidad tenderá a alcanzar un valor máximo para luego disminuir a medida que aumenta el número de onda. Es evidente que para un modo con $k>>L_{u}^{-1}\left(\lambda<<L_{u}\right)$ las interfases difusas se comportarían como si estuviesen a distancias muy grandes, siendo la interacción entre ellas muy débil y, en consecuencia, muy pequeña la tasa de crecimiento de la inestabilidad $\sigma$.

A continuación, utilizaremos dos métodos numéricos diferentes, dependientes de la hipótesis espacial utilizada para resolver las ecuaciones linealizadas de Euler alrededor del flujo base. La primera formulación se basa en un método espectral de colocación de Chebyshev, cuando la geometría es simplificada a 1D, y el segundo se construye en una formulación variacional 2D de las ecuaciones, utilizando una discretización de segundo orden de elementos finitos (FEM) de tipo Taylor-Hood cuadrática en densidad.

\subsubsection{Formulación 1D}

El dominio computacional $\Omega 1 \mathrm{D}$ que vamos a utilizar sería $y \in[-H, H]$ con $N$ puntos discretos. Resolveremos el problema de autovalores desde el punto de vista de una formulación velocidad-presión-densidad, que aparece planteada en la expresión (2.111), donde solamente impondremos condiciones de contorno o frontera homogéneas tipo Dirichlet para la velocidad vertical y la derivada normal de la velocidad vertical de las perturbaciones de la forma

$$
\hat{v}(y= \pm H)=0 \quad \mathrm{D} \hat{v}(y= \pm H)=0
$$

\subsubsection{Formulación 2D}

Utilizaremos un dominio computacional 2D $\Omega=[-L, L] \mathrm{x}[-H, H]$ en el plano $x y$ con condiciones de contorno homogéneas tipo Dirichlet para las amplitudes de las componentes en $x$ y en $y$ de la velocidad perturbada $\hat{u} \mathrm{y} \hat{v}$, respectivamente, en las fronteras superior e inferior del dominio, $\mathrm{y}$ 


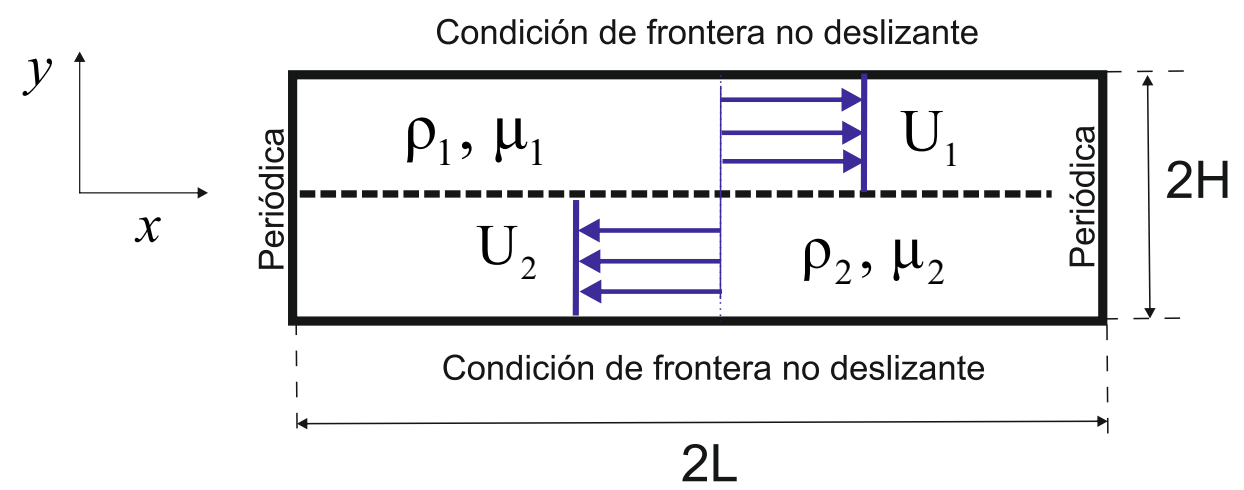

Figura 4.4: Representación de la geometría utilizada para un análisis 2D. La línea discontinua representa la coordenada $y=0$. Las condiciones de contorno para las perturbaciones son establecidas también en cada frontera.

condiciones periódicas en las fronteras derecha e izquierda. La figura que representa el dominio $\Omega$ es la figura 4.4 .

Así, el problema de partida se centra en la resolución del problema BiGlobal de autovalores dado por (2.46) sin necesidad de recurrir a la suposición periódica en la dirección horizontal $x$ de infinita longitud. La perturbación del flujo debe satisfacer las condiciones de frontera no deslizantes $\mathbf{u}=0$ sobre $y= \pm H$. Es decir

$$
\begin{gathered}
\hat{u}(-L, y)=\hat{u}(L, y) \\
\hat{v}(-L, y)=\hat{v}(L, y) \\
\hat{u}(x, \pm H)=0 \\
\hat{v}(x, \pm H)=0
\end{gathered}
$$

Con objeto de discretizar las ecuaciones, vamos a utilizar una malla no estructurada de elementos triangulares la cual se muestra en la figura 4.7. Resolveremos el problema de autovalores mediante el método de Arnoldi usando una iteración del subespacio de Krylov [117], [110]. A fin de comprobar la precisión de los resultados durante el análisis de estabilidad, 
incrementaremos el número de nodos de la malla hasta que los tres dígitos significativos del modo más inestable lleguen a converger.

\section{2. $\quad$ Resultados}

Para un valor fijo del número de onda $k$, el problema de autovalores dado por las expresiones 2.111) (en 1D) y 2.74 (en 2D) es numéricamente resuelto utilizando las respectivas condiciones de frontera 4.7) (1D) y (4.8)-4.11) (2D), respectivamente, obteniéndose, así, los autovalores y las correspondientes autofunciones. Un método espectral de colocación de puntos de Chebyshev y un método en elementos finitos FEM son utilizados para los dominios $1 \mathrm{D}$ y $2 \mathrm{D}$, respectivamente.

\subsubsection{Validación de la formulación 1D}

Como previa validación del problema 1D, estudiaremos el límite $L_{\rho} \rightarrow 0$ y $L_{u} \rightarrow 0$, i.e., el caso clásico de la KHI. Así, validaremos la solución obtenida para el flujo base dado por (4.3). Cada autovalor lo podemos expresar como un número complejo de la forma $\gamma=\sigma+i \omega$. Los autovalores numéricos se han obtenido para un número de puntos de Gauss-Lobatto $N=256$. Por otro lado, el resultado analítico clásico normalizado del autovalor $\gamma_{C}$ vendrá dado por 86 ]

$$
\gamma_{C}=-i A_{\rho} k \pm k \sqrt{1-A_{\rho}^{2}}
$$

Utilizando el flujo base definido por (4.3), la parte superior de la figura 4.5 muestra la tasa de crecimiento lineal de la inestabilidad normalizada $\sigma$ en función del número de onda normalizado $k$ para diferentes números de Atwood $A_{\rho}$. Los resultados numéricos se han obtenido para valores pequeños de los parámetros que caracterizan los espesores de las capas de transición de densidad y velocidad $L_{\rho}=10^{-3}$ y $L_{u}=10^{-3}$, respectivamente, caso que se corresponde con una transición muy abrupta entre ambos fluidos. Los autovalores numéricos de $\sigma$ son comparados con la parte real de la expresión analítica (4.12), obteniéndose un buen acuerdo entre la solución analítica y la numérica.

Asimismo, para el referido flujo base dado por 4.3 también se ha representado en la parte inferior de la figura 4.5 la frecuencia normalizada $\omega$ de la perturbación frente al número de onda normalizado $k$ para los parámetros anteriormente mencionados. Es decir, para diferentes valores del número de 

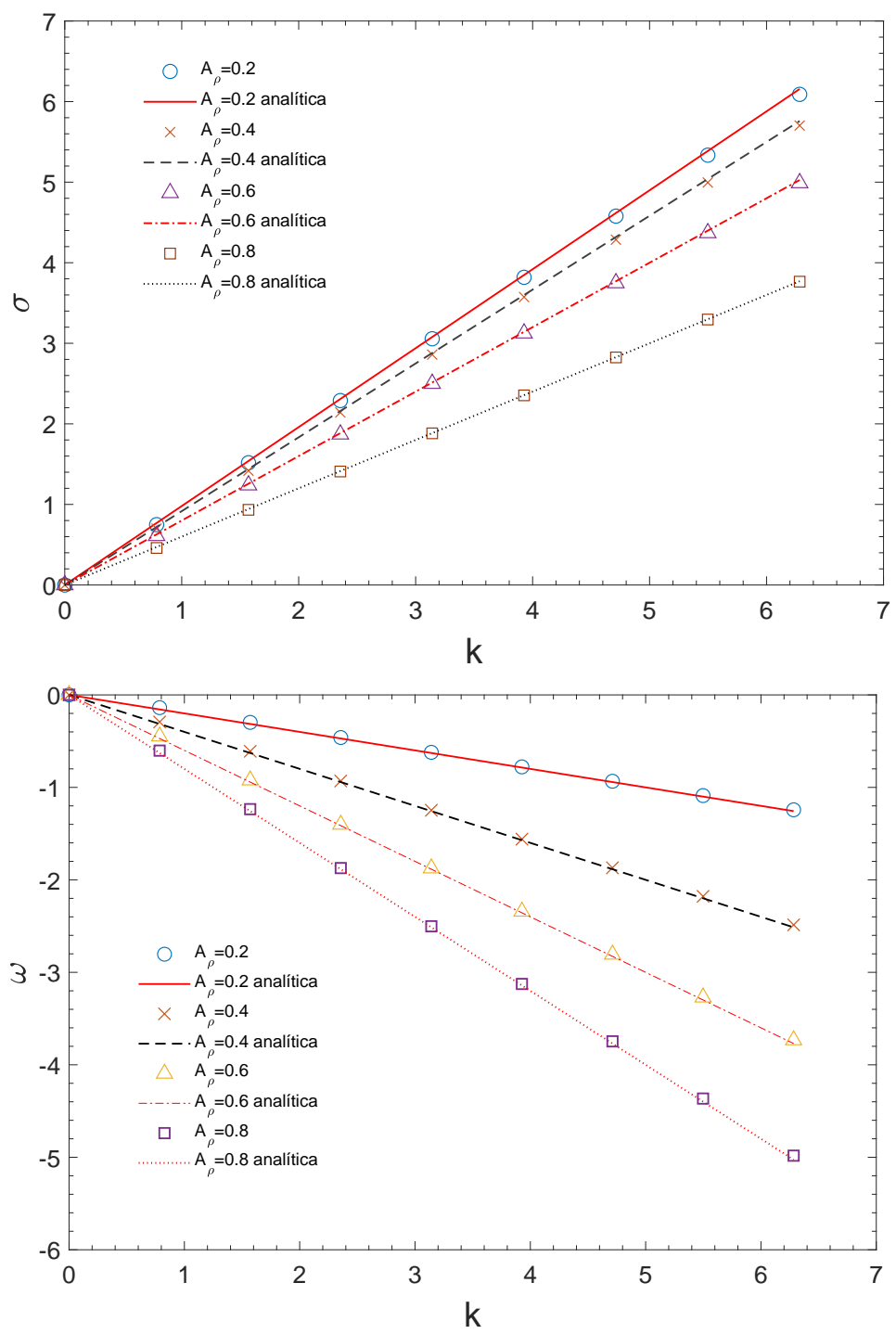

Figura 4.5: Tasa de crecimiento de la inestabilidad $\sigma$ (gráfica superior) y frecuencia $\omega$ (gráfica inferior) frente al número de onda $k$ para diferentes valores del número de Atwood $A_{\rho}=0.2,0.4,0.6,0.8$ para $H=1, L_{\rho}=$ $L_{u}=10^{-3}$ y el flujo base dado por 4.3 . Mediante líneas se ha representado la expresión analítica 4.12 para establecer una comparativa. 
Atwood $A_{\rho}=0.2,0.4,0.6,0.8, H=1, L_{\rho}=L_{u}=10^{-3}$. En la misma gráfica se ha establecido una comparativa entre los valores numéricos y los analíticos correspondientes a la parte imaginaria de 4.12 obteniéndose también un buen acuerdo.

\subsubsection{Efecto del espesor de la capa de cortadura en la KHI}

En este apartado analizaremos, para un valor fijo de $A_{\rho}, H$ y $L_{\rho}$, el efecto del parámetro $L_{u}$ sobre la KHI, es decir, el parámetro que caracteriza la anchura de la capa de transición de la velocidad (capa de cortadura). Este análisis lo haremos tanto desde el punto de vista de una formulación 1D, así como $2 \mathrm{D}$.

\section{Formulación 1D}

El punto de partida se sitúa en el análisis de estabilidad del flujo base definido en la expresión (4.3). En la gráfica 4.6 hemos representado la tasa de crecimiento de la inestabilidad $\sigma$ frente $k$ para $L_{\rho}=10^{-3}, H=1$ y $A_{\rho}=0.2$ utilizando dicho flujo base (4.3). Los cálculos numéricos se han realizado para $L_{u}=0.2,0.1,0.08,0.06,0.05,0.04$. En dicha gráfica se observa que, para cada $L_{u}$, la tasa de crecimiento de la inestabilidad tiene siempre un máximo $\sigma_{\max }$ en un valor determinado del número de onda normalizado $k_{\max }$. Así, por ejemplo, para $L_{u}=0.2$ el máximo de la curva $\sigma_{\max }=0.983$ se obtiene en $k_{\max }=2.8$. Al aumentar el valor del parámetro $L_{u}$, el máximo de la curva $\sigma_{\max }$ se incrementa y se alcanza para un número de onda mayor $k_{\max }$.

Por tanto, de los resultados obtenidos podemos verificar que el espesor de la capa de cortadura tiene un efecto estabilizante sobre la KHI, que es mayor a medida que aumenta $L_{u}$, tal y como habían encontrado con anterioridad Guzdar et al. [100], Wang et al. [93, [101, Ye et al. [102, Gan et al. 94] y G. A. Hoshoudy y Huseyin Cavus [96]. Adicionalmente, nuestro estudio pone en evidencia, para cada $L_{u}$, la existencia de un número de onda $k$ para el cual la tasa se crecimiento de la inestabilidad $\sigma$ es máxima. La justificación de este comportamiento de la relación de dispersión, que ya habíamos adelantado en el apartado de la descripción del flujo base, se puede explicar en términos de interacción entre las dos interfases difusas de vorticidad: para modos con $k>>L_{u}^{-1}$, la interacción se debilita, disminuyendo $\sigma$ con $k$. 


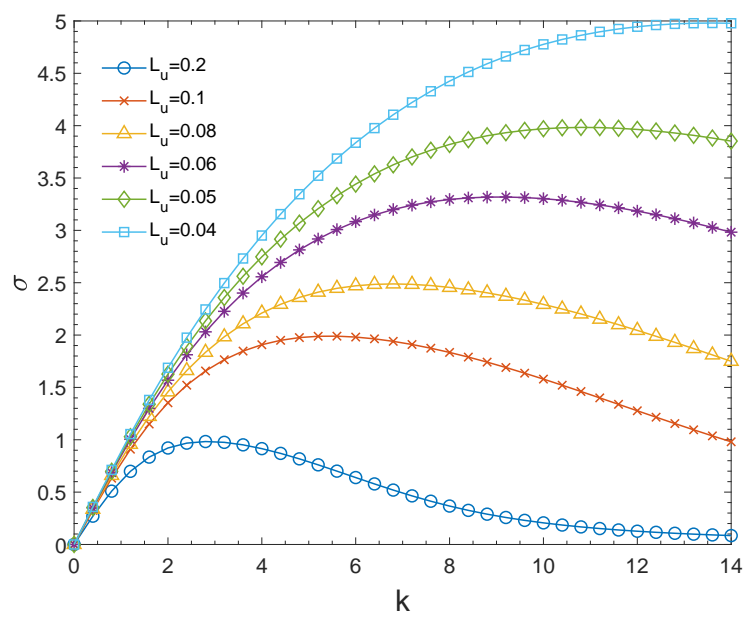

Figura 4.6: Tasa de crecimiento normalizada $\sigma$ de la KHI pura frente al número de onda normalizado $k$ para $A_{\rho}=0.2, L_{\rho}=10^{-3}, U_{o}=1, H=1 \mathrm{y}$ diferentes valores de $L_{u}$.

\section{Formulación 2D}

El cálculo numérico 2D lo realizaremos exclusivamente para el flujo base definido por 4.3 y en un dominio de computación definido por un rectángulo de dimensiones $2 L$ y $2 H$. La discretización la llevaremos a cabo por elementos finitos (FEM), utilizando elementos triangulares de Taylor-Hood. La perturbación de la densidad la aproximaremos por polinomios de segundo orden. Todos los cálculos los llevaremos a cabo en el dominio computacional $H=1$ y $L=4$.

Hemos utilizado en nuestros cálculos dos mallas a las que denominaremos malla 1 y malla 2 . La malla 1 tiene un número de nodos cuadráticos de malla y elementos triangulares de 23145 y 11458, respectivamente, mientras que la malla 2, más refinada que la anterior, tiene 34026 nodos cuadráticos de malla y 68219 elementos triangulares. Ambas mallas han sido refinadas en la línea horizontal $y=0$ (ver figura 4.7 relativa a la malla 1 ).

En primer lugar, analizaremos la KHI clásica para una interfase abrupta. Este estudio lo realizaremos para el caso $A_{\rho}=0.2, L_{\rho}=L_{u}=10^{-2}$, $U_{o}=1, H=1$. Las ecuaciones numéricas que resolveremos mediante el FEM contienen un parámetro de control $\chi$ relacionado con el inverso de una pseudo-viscosidad o viscosidad artificial. Es evidente que cuando $\chi \rightarrow \infty$, estaríamos ante el caso de flujo no viscoso que es el que tratamos de re- 
Capítulo 4. Inestabilidad de Kelvin-Helmholtz (KHI) en un

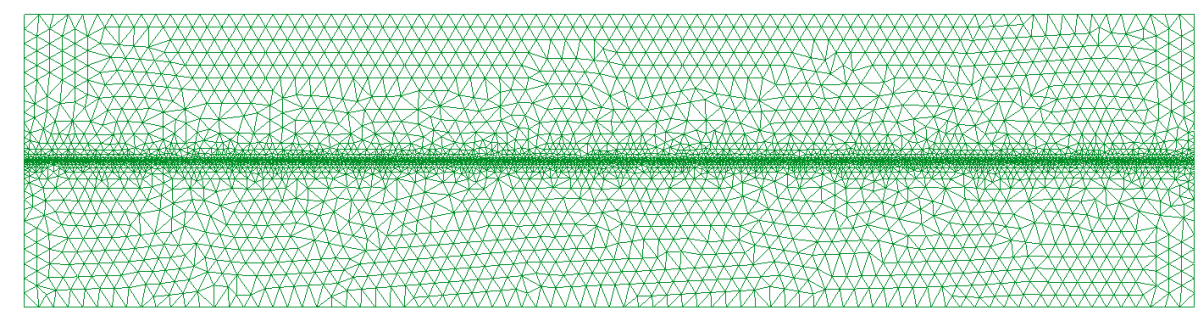

Figura 4.7: Malla utilizada para el análisis en 2D.

solver. Por ello, nos encontramos ante la necesidad de realizar un estudio de convergencia en función del parámetro $\chi$. Este estudio lo realizaremos para la malla 2 y para varios números de onda $k$. En la tabla 4.1 se resume el estudio de convergencia observándose la tendencia esperada: a medida que aumentamos el valor del parámetro $\chi$, los autovalores se acercan a los obtenidos utilizando el método espectral 1D siendo el flujo base no viscoso.

A continuación, vamos a calcular un mayor número de autovalores mediante el FEM (modelo 2D) para la transición de flujo base abrupta, que los validaremos con los obtenidos en el método espectral (modelo 1D). Por ello, en la gráfica de la parte superior de la figura 4.8 se ha representado la tasa de crecimiento de la inestabilidad normalizada $\sigma$ frente al número de onda normalizado $k$. Por otro lado, la representación de la frecuencia normalizada $\omega$ frente al número de onda $k$ se puede observar en la gráfica inferior de figura 4.8. De la observación de ambas gráficas, se desprende un buen acuerdo de los resultados obtenidos en la formulación 2D utilizando como referencia la $1 \mathrm{D}$.

En la figura 4.9 hemos representado las autofunciones de todas las variables perturbadas del fluido para el modo con número de onda $k_{x}=5 \pi / 4$. Se aprecia claramente la estructura periódica de las autofunciones con una longitud de onda definida $\lambda_{x}=2 \pi / k_{x}=8 / 5$.

Asimismo, vamos a representar en la figura 4.10 las líneas de corriente para el modo cuyas autofunciones se han representado en la figura 4.9. En dicha figura se observan cinco vórtices con vorticidad negativa (líneas de corriente de color rojo) y cuatro vórtices con vorticidad positiva (líneas de corriente de color azul).

En segundo lugar, vamos a analizar el rol de una transición difusa del flujo base en velocidad a través de la superficie de separación entre las dos fases tomando como referencia los parámetros anteriores, es decir, $A_{\rho}=0.2$ 

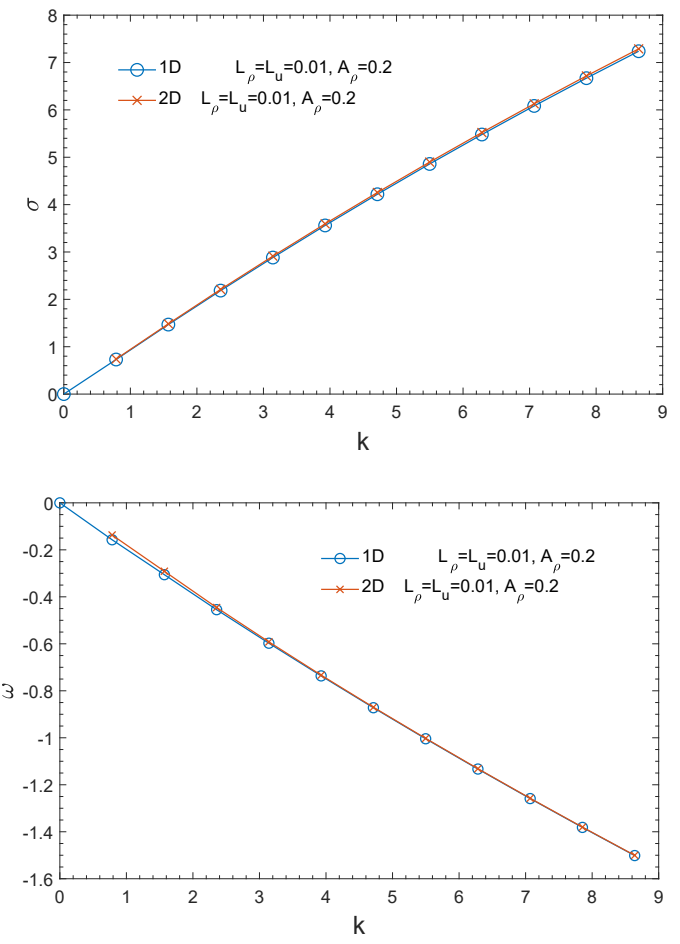

Figura 4.8: Tasa de crecimiento de la KHI $\sigma$ (gráfica superior) y frecuencia $\omega$ (gráfica inferior) frente al número de onda normalizado $k$ para $A_{\rho}=0.2$, $L_{\rho}=L_{u}=10^{-2}, H=1, U_{o}=1$. 
Capítulo 4. Inestabilidad de Kelvin-Helmholtz (KHI) en un
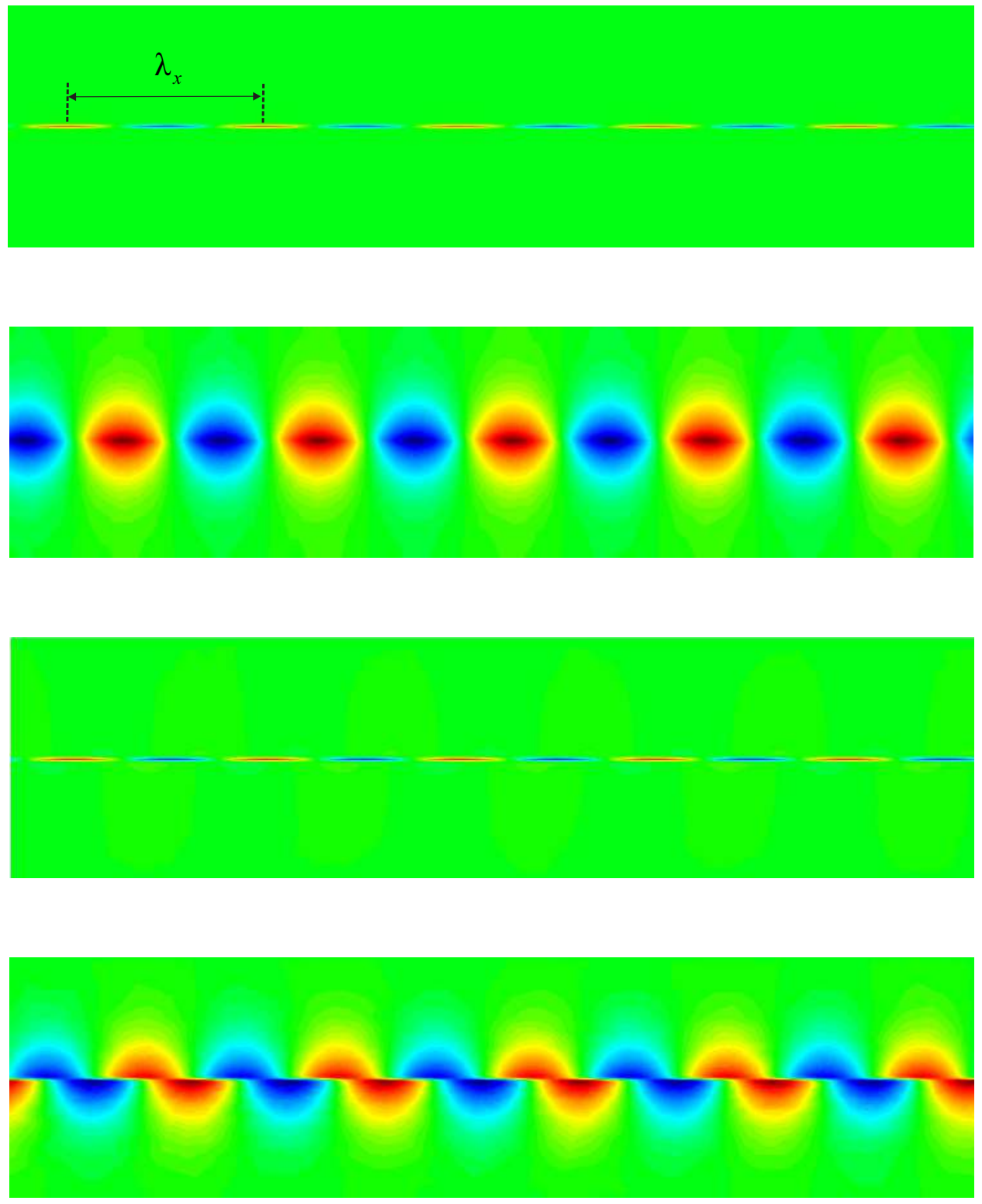

Figura 4.9: Modo más inestable de la KHI para el caso $k=5 \pi / 4, H=1$, $L=4$ y $A_{\rho}=0.2$. Desde la gráfica superior a la inferior, amplitud de la perturbación de densidad, presión, velocidad horizontal y velocidad vertical. Los cálculos se han realizado con los parámetros de interfase $L_{s}=L_{u}=0.01$. 
Tabla 4.1: Estudio de convergencia para diferentes números de onda $k$

\begin{tabular}{lccc}
\hline \hline$k$ & $\chi$ & $\sigma$ & $\omega$ \\
\hline$\pi$ & $10^{4}$ & 2.56098984 & -0.62308099 \\
$\pi$ & $10^{5}$ & 2.85647632 & -0.59977738 \\
$\pi$ & $2 \cdot 10^{5}$ & 2.88231404 & -0.59746523 \\
\hline $5 \pi / 4$ & $10^{4}$ & 3.20495458 & -0.76268807 \\
$5 \pi / 4$ & $10^{5}$ & 3.53339313 & -0.73886399 \\
$5 \pi / 4$ & $2 \cdot 10^{5}$ & 3.56007084 & -0.73655339 \\
\hline $7 \pi / 4$ & $10^{4}$ & 4.44849129 & -1.03077459 \\
$7 \pi / 4$ & $10^{5}$ & 4.83058051 & -1.00645672 \\
$7 \pi / 4$ & $2 \cdot 10^{5}$ & 4.85849868 & -1.00420040 \\
\hline $2 \pi$ & $10^{4}$ & 5.09981979 & -1.15006484 \\
$2 \pi$ & $10^{5}$ & 5.45160650 & -1.13527634 \\
$2 \pi$ & $2 \cdot 10^{5}$ & 5.48003196 & -1.13305849 \\
\hline $9 \pi / 4$ & $10^{4}$ & 5.67171073 & -1.27548284 \\
$9 \pi / 4$ & $10^{5}$ & 6.05470978 & -1.26096052 \\
$9 \pi / 4$ & $2 \cdot 10^{5}$ & 6.08359859 & -1.25878886 \\
\hline $5 \pi / 2$ & $10^{4}$ & 6.22613594 & -1.39780213 \\
$5 \pi / 2$ & $10^{5}$ & 6.64021298 & -1.38363608 \\
$5 \pi / 2$ & $2 \cdot 10^{5}$ & 6.66953424 & -1.38151920 \\
\hline $11 \pi / 4$ & $10^{4}$ & 6.76316533 & -1.51722167 \\
$11 \pi / 4$ & $10^{5}$ & 7.20842856 & -1.50342556 \\
$11 \pi / 4$ & $2 \cdot 10^{5}$ & 7.238160751 & -1.50137211 \\
\hline & & &
\end{tabular}

y $L_{\rho}=0.01$. En nuestro caso, tomaremos el valor de $L_{u}=0.1$. En la gráfica superior de la figura 4.11 se muestra la tasa de crecimiento de la inestabilidad normalizada $\sigma$ frente al número de onda normalizado $k$ y en la gráfica inferior de la figura 4.11 la frecuencia normalizada $\omega$ frente al número de onda $k$.

Con respecto al aspecto de las autofunciones de todas las variables perturbadas, las gráficas de las mismas y para el modo de número de onda $k_{x}=5 \pi / 4$ se encuentran en la figura 4.12 . En dichas autofunciones se aprecia una periodicidad con una longitud de onda definida $\lambda_{x}=2 \pi / k_{x}=4$. 


\section{Capítulo 4. Inestabilidad de Kelvin-Helmholtz (KHI) en un}

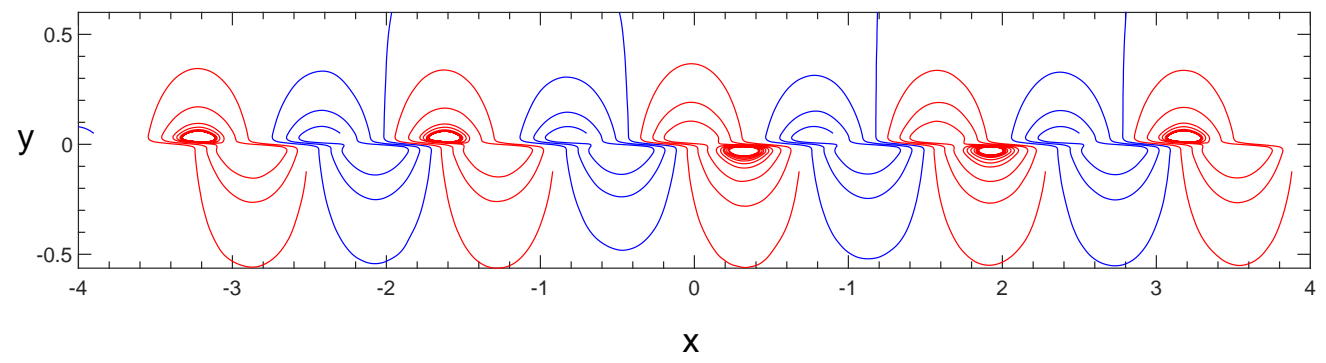

Figura 4.10: Líneas de corriente de la perturbación para $k=5 \pi / 2, A_{\rho}=0.2$, $H=1, U_{o}=1, L_{\rho}=0.01, L_{u}=0.01$.

Asimismo, sería de interés representar en la figura 4.13 las líneas de corriente para el modo cuyas autofunciones se han representado en la figura 4.12. En dicha figura se observan cinco vórtices con vorticidad negativa (líneas de corriente de color rojo) y cuatro vórtices con vorticidad positiva (líneas de corriente de color azul).

Si comparamos las autofunciones de las figuras 4.12 y 4.9, para la transición difusa $L_{u}=0.1$ y abrupta de velocidad $L_{u}=0.01$, respectivamente, y que se han obtenido para el mismo número de onda $k_{x}=5 \pi / 4$ e idénticos parámetros $H=1, L=4, A_{\rho}=0.2$ y $L_{\rho}=0.01$, se muestran pocas variaciones con respecto al modo de la densidad perturbada. Sin embargo, el aspecto de los modos de presión perturbada, velocidad horizontal y vertical muestran sustanciales diferencias. Es significativo observar la desaparición de la cizalla en el modo de la velocidad perturbada vertical.

A modo de ilustración y para el caso de la transición difusa en velocidad, se muestran en la gráfica 4.14 las autofunciones de todas las variables perturbadas del modo $k=\pi / 2$, siendo $H=1, L=4, A_{\rho}=0.2, L_{\rho}=0.01$ y $L_{u}=0.1$ representándose las líneas de corriente en la gráfica 4.15 . Se observan cuatro vórtices: dos de ellos con vorticidad positiva (líneas de color azul) y otros dos con vorticidad negativa (líneas de color rojo).

En tercer y último lugar, podemos analizar el caso de transición abrupta en velocidad $L_{u}=0.01$, pero difusa en densidad $L_{\rho}=0.3$ siendo $A_{\rho}=0.2$. En la gráfica superior de la figura 4.16 se muestra la tasa de crecimiento de la inestabilidad normalizada $\sigma$ frente al número de onda normalizado $k \mathrm{y}$ en la gráfica inferior de la figura 4.16 la frecuencia normalizada $\omega$ frente al número de onda $k$.

Para el modo $k_{x}=6 \pi / 4$ se han representado en la figura 4.17 las autofunciones de todas las variables perturbadas. En dichas autofunciones se aprecia 

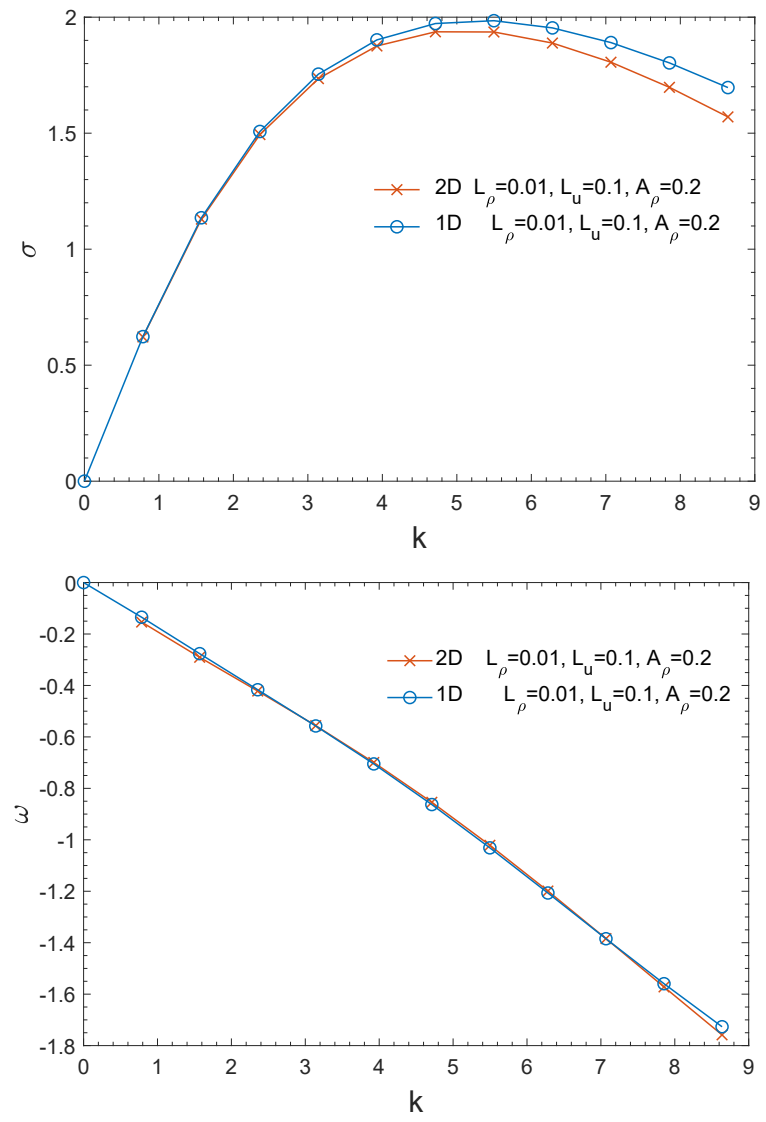

Figura 4.11: Tasa de crecimiento $\sigma$ de la KHI (gráfica superior) y frecuencia $\omega$ (gráfica inferior) frente al número de onda normalizado $k$ para $A_{\rho}=0.2$, $L_{\rho}=0.01, H=1, L_{u}=0.1, U_{o}=1$. 
Capítulo 4. Inestabilidad de Kelvin-Helmholtz (KHI) en un
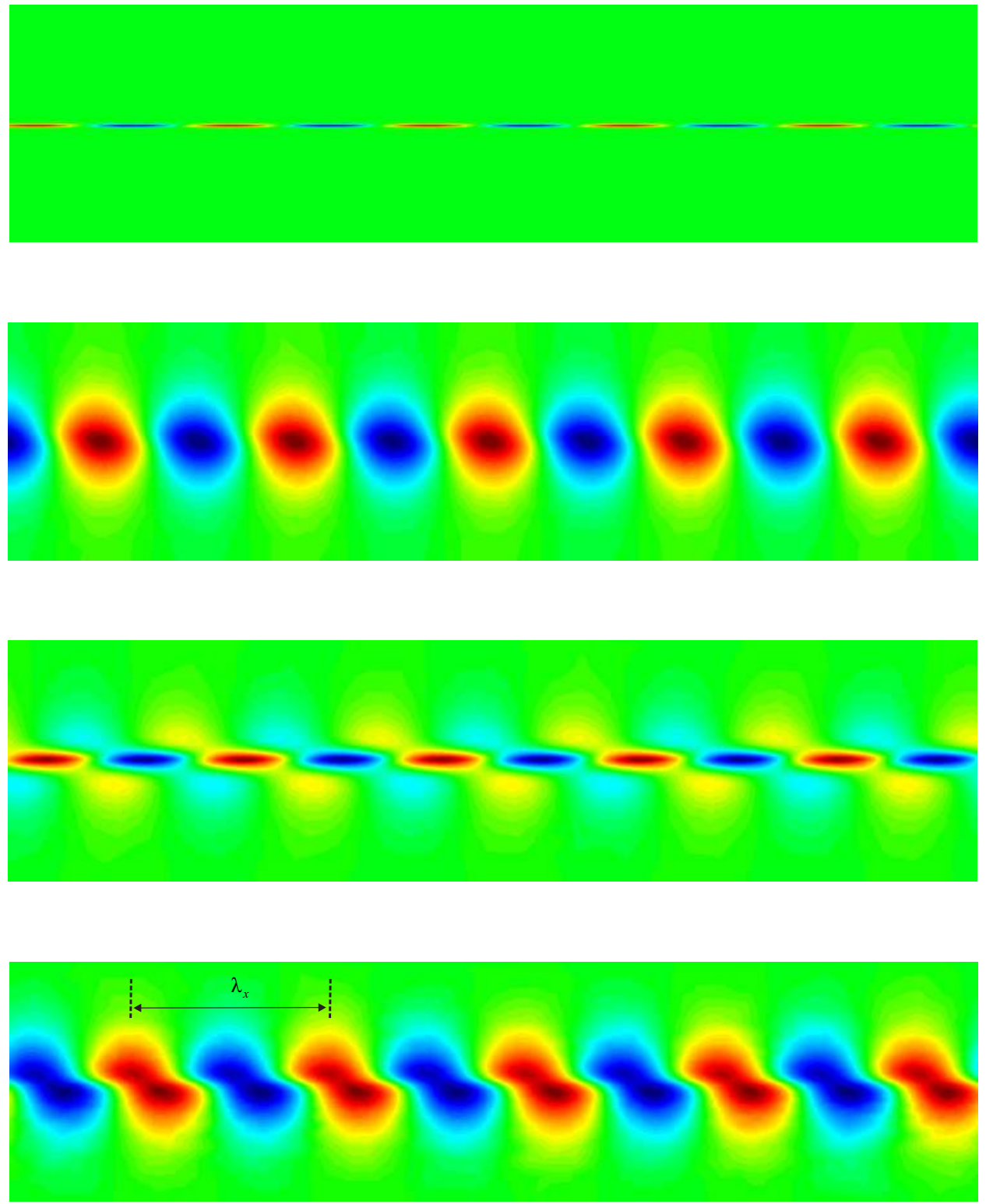

Figura 4.12: Perturbación más inestable de la KHI pura el caso $k=5 \pi / 4$, $H=1, L=4$ y $A_{\rho}=0.2$. Desde la gráfica superior a la inferior, autofunciones de densidad, presión, velocidad horizontal y velocidad vertical. Los cálculos fueron llevados a cabo con los parámetros de interfase $L_{\rho}=0.01 \mathrm{y}$ $L_{u}=0.1$. 


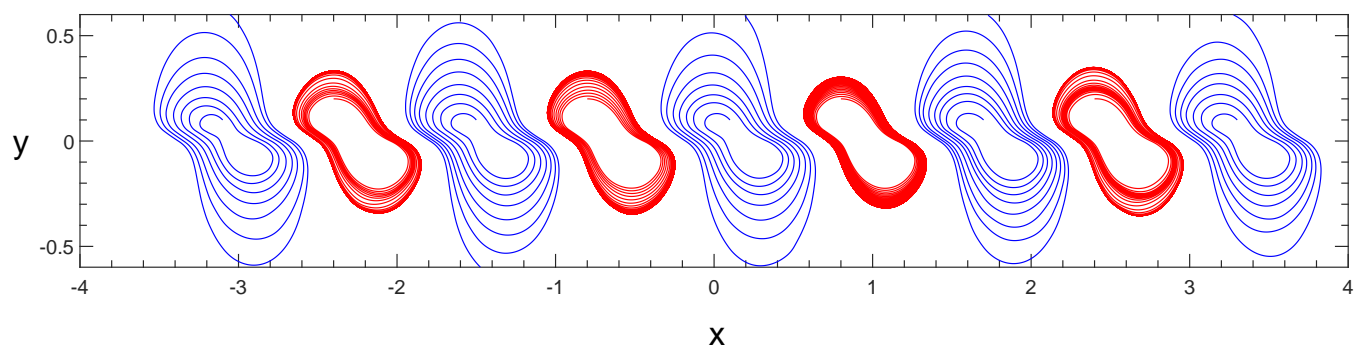

Figura 4.13: Líneas de corriente para $k=5 \pi / 2, A_{\rho}=0.2, H=1, U_{o}=1$, $L_{\rho}=0.01, L_{u}=0.1$.

una periodicidad con una longitud de onda definida $\lambda_{x}=2 \pi / k_{x}=2 / 3$.

\subsubsection{Evolución conjunta de KHI y RTI}

En este apartado analizaremos numéricamente el caso de la evolución conjunta de la KHI y la RTI. Desde el punto de vista de Guzdar et al. [100, este caso es semejante a estudiar el efecto sobre la RTI de una velocidad de cortadura 1

\section{Formulación 1D}

Los cálculos numéricos que hemos llevado a cabo utilizando la formulación 1D previamente mencionada se muestran en la figura 4.18. Estos cálculos se han realizado para $A_{\rho}=0.6, H=1, L_{\rho}=L_{u}=0.1,0.3,0.5 \mathrm{y}$ $U_{o}=1$.

En cada una de las cuatro imágenes de la figura 4.18 se verifica lo observado por Wang et al. [101] y Ye et al. [102, en tanto en cuanto un aumento de $L_{u}=L_{\rho}$ produce un efecto estabilizante. Asimismo, se observa también lo encontrado por Guzdar et al. [100, i.e., un máximo cuya existencia está asociada a una zona de transición difusa en velocidad cuyo perfil viene dado por (4.3). Es decir, el efecto de espesor finito de la referida zona de transición en velocidad hace posible que la tasa de crecimiento de la inestabilidad $\sigma$ del modo más inestable no crezca monótonamente en función del número de onda $k$, sino que presente un máximo para decrecer posteriormente monótonamente hasta alcanzar un número de onda crítico $k_{c r}$ por encima del cual

\footnotetext{
${ }^{1}$ De hecho, Guzdar et al. [100] utilizan la denominación de RTI generalizada para el caso en el que la RTI esté afectada por una velocidad de cortadura
} 
Capítulo 4. Inestabilidad de Kelvin-Helmholtz (KHI) en un
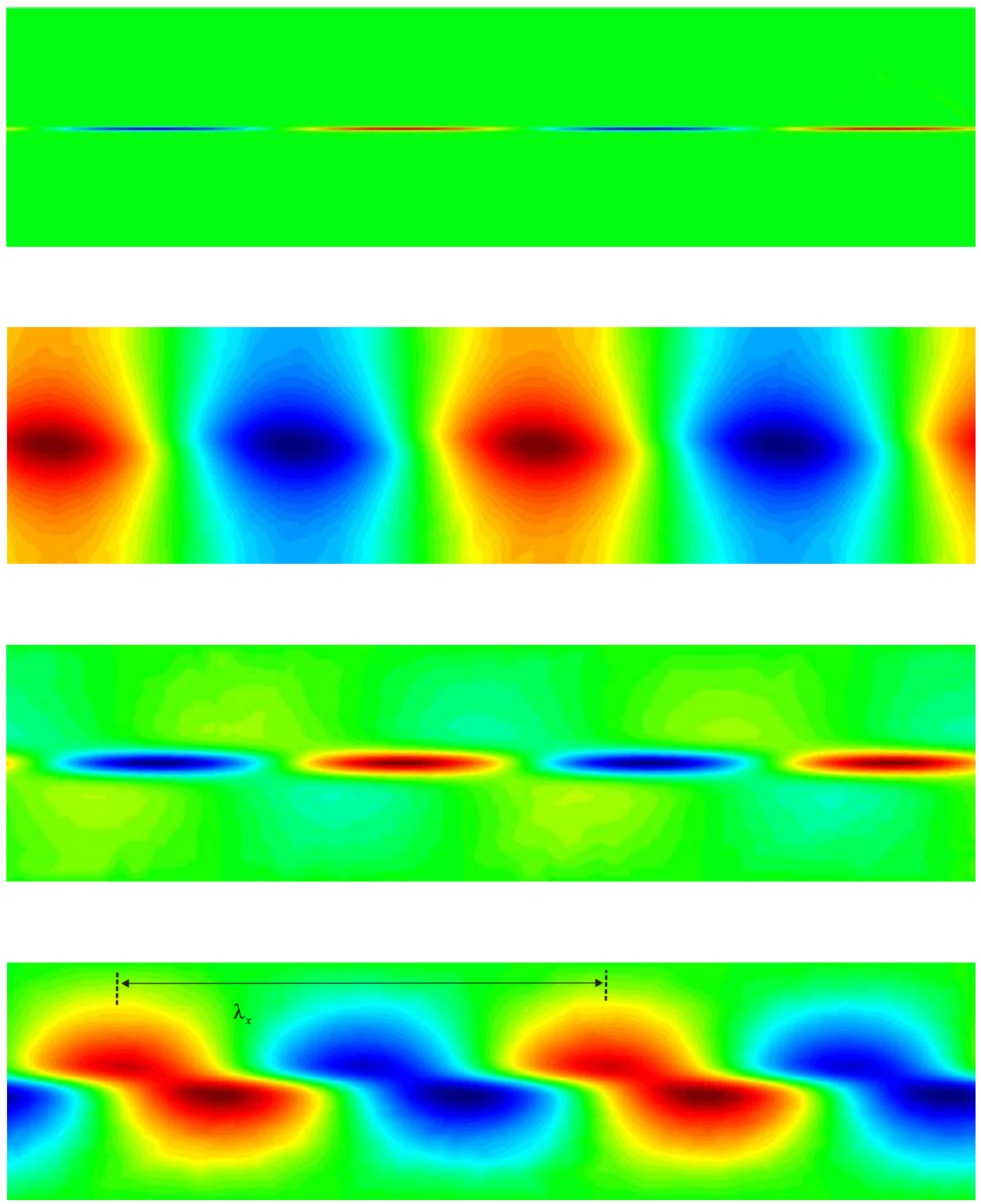

Figura 4.14: Modo más inestable de la KHI para el caso $k=\pi / 2, H=1$, $L=4$ y $A_{\rho}=0.2$. Desde la gráfica superior a la inferior, se muestran las amplitudes de las perturbaciones en densidad, presión, velocidad horizontal y velocidad vertical. Los cálculos fueron llevados a cabo con los parámetros de interfase $L_{\rho}=0.01$ y $L_{u}=0.1$. 


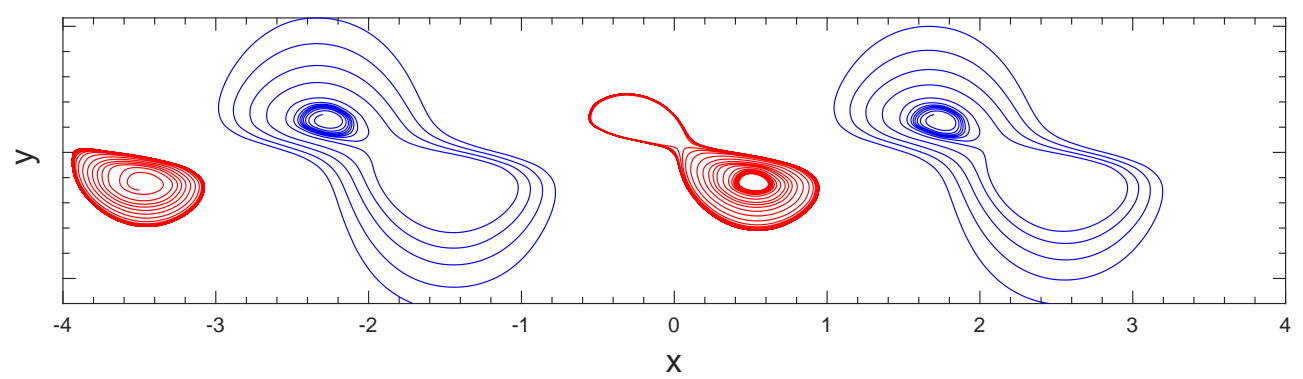

Figura 4.15: Líneas de corriente para $k=\pi / 2, A_{\rho}=0.2, H=1, U_{o}=1$, $L_{\rho}=0.01, L_{u}=0.1$. Se aprecian dos vórtices con vorticidad positiva (línea de color azul) y dos con vorticidad negativa (línea de color rojo).

el modo deja de existir: por ejemplo, para la rama $L_{u}=L_{\rho}=0.2, k_{c r} \simeq 6$. De las gráficas se observa que el rango de existencia del modo más inestable disminuye conforme $L_{u}$ aumenta.

Por último, con objeto de analizar la coexistencia de las dos inestabilidades en el caso que nos ocupa, en la figura 4.19 se ha representado la tasa de crecimiento normalizada de la inestabilidad $\sigma$ frente al número de onda normalizado $k$ para $A_{\rho}=0.6, H=1, L_{\rho}=L_{u}=0.1,0.2,0.3,0.5$, $U_{o}=1$. En la gráfica de la parte superior izquierda, la relación de dispersión para el caso de solo RTI interseca con la correspondiente a la coexistencia simultánea de RTI y KHI. La intersección se produce para el número de onda $k^{*} \simeq 8.235$. Se aprecia que cuando $k>k^{*}, \sigma_{R T+K H I}<\sigma_{R T}$, i.e. la velocidad de cortadura tiene un efecto estabilizante sobre la RTI; cuando $k<k^{*}, \sigma_{R T+K H I}>\sigma_{R T}$, el efecto ahora es desestabilizante. Observando el resto de gráficas se comprueba que a medida que aumenta $L_{u}, k^{*}$ disminuye, aumentando el rango en el que $\sigma_{R T+K H I}<\sigma_{R T}$, i.e., el rango en el que el modo RTI es atenuado por la acción de la velocidad de cortadura.

\section{Formulación 2D}

En las gráficas de la figura 4.20 se muestran la tasa de crecimiento de la inestabilidad $\sigma$ (gráfica superior) y la frecuencia $\omega$ (gráfica inferior), respectivamente, versus el número de onda $k$. En dichas gráficas se han representado los resultados obtenidos mediante la formulación 1D con objeto de compararlos con los de la formulación 2D obteniéndose un buen acuerdo.

Por otro lado, se han representado las autofunciones de las variables perturbadas para el caso $L_{\rho}=L_{u}=0.2, H=1, A_{\rho}=0.6$ y $k_{x}=\pi$ en la 
Capítulo 4. Inestabilidad de Kelvin-Helmholtz (KHI) en un 102 medio heterogéneo
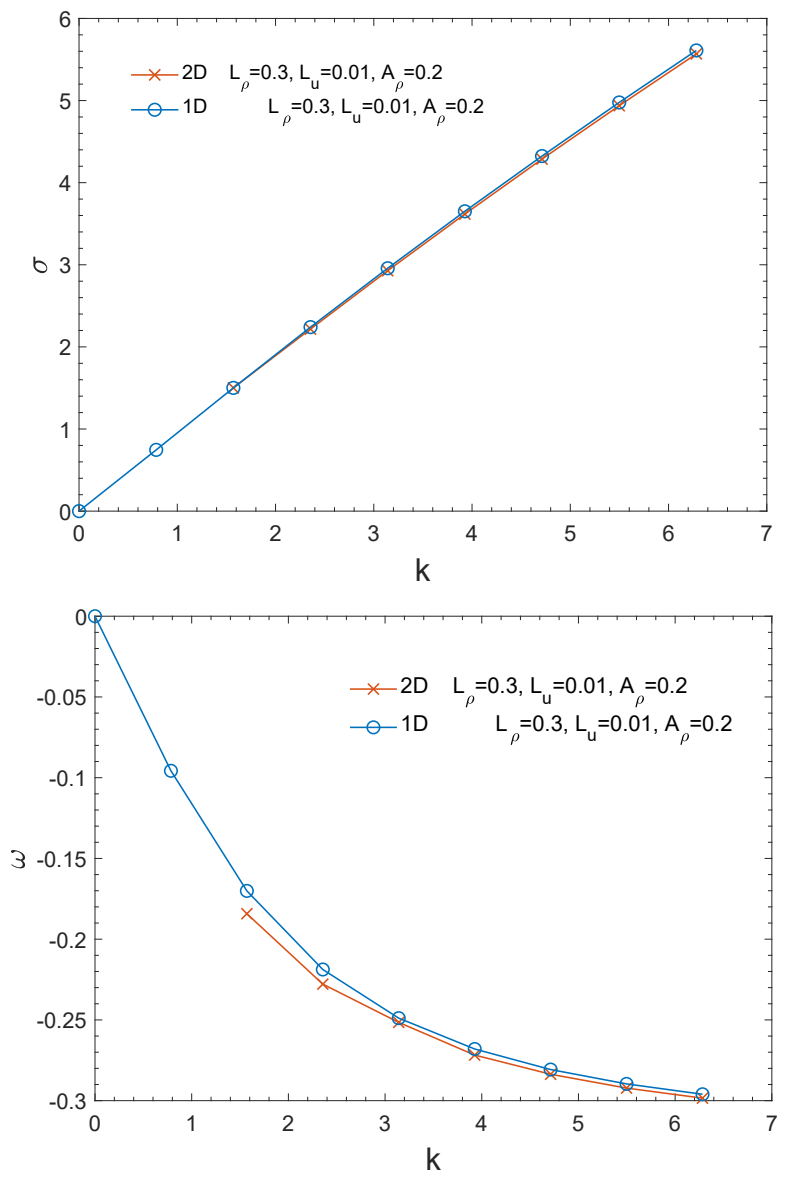

Figura 4.16: Tasa de crecimiento de la KHI $\sigma$ (gráfica superior) y frecuencia $\omega$ (gráfica inferior) frente al número de onda normalizado $k$ para $A_{\rho}=0.2$, $L_{\rho}=0.3, H=1, L_{u}=0.01, U_{o}=1$. 

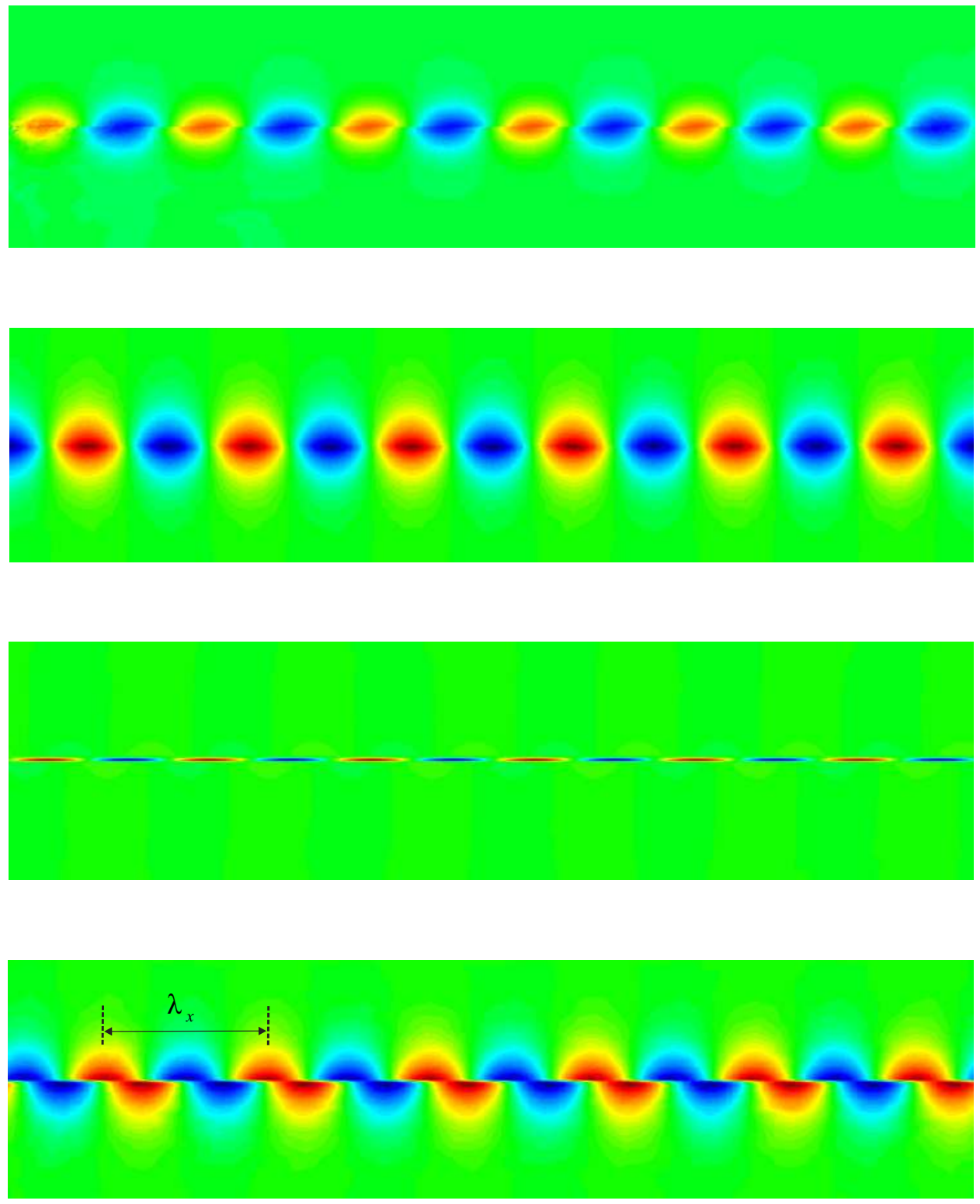

Figura 4.17: Modo más inestable de la KHI para el caso $k=6 \pi / 4, H=1$, $L=4$ y $A_{\rho}=0.2$. Desde la gráfica superior a la inferior, se muestran las amplitudes de las perturbaciones en densidad, presión, velocidad horizontal y velocidad vertical. Los cálculos fueron llevados a cabo con los parámetros de interfase $L_{\rho}=0.3$ y $L_{u}=0.01$. 
Capítulo 4. Inestabilidad de Kelvin-Helmholtz (KHI) en un

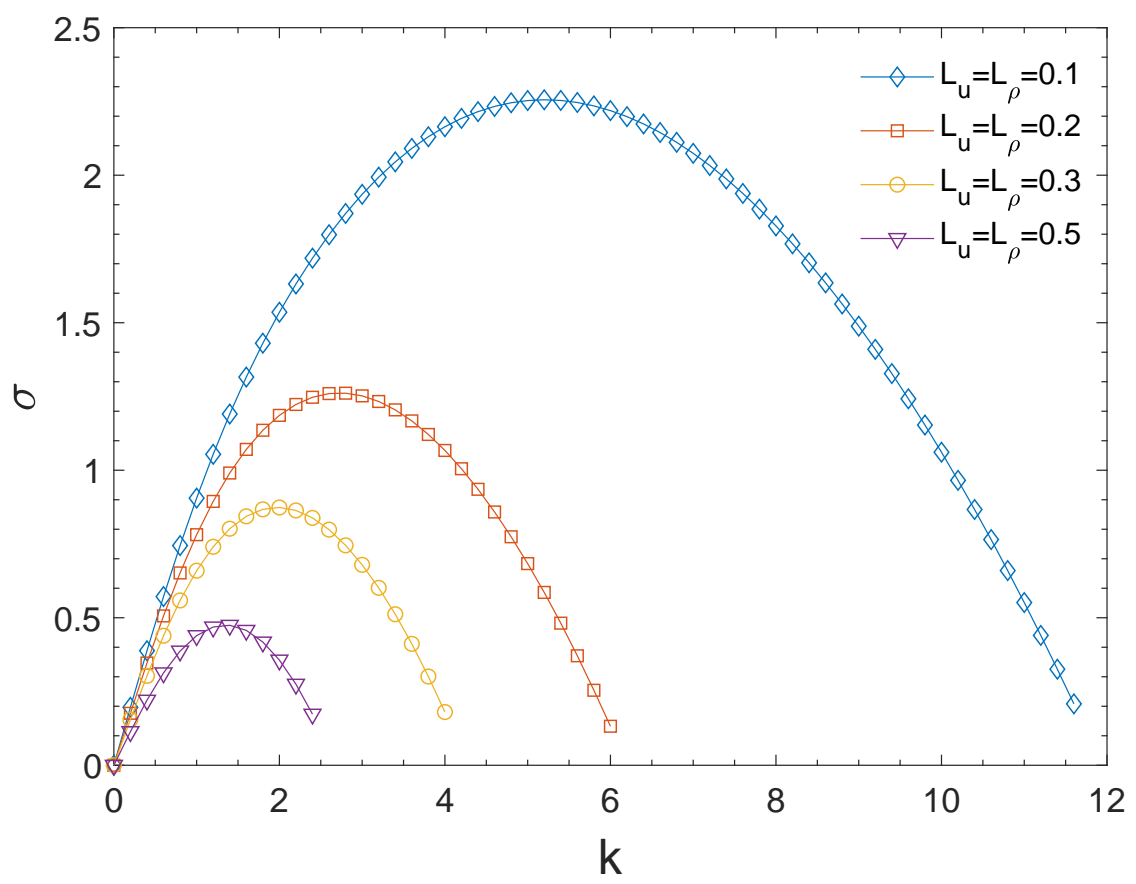

Figura 4.18: Tasa de crecimiento normalizada de la inestabilidad $\sigma$ (RTI+KHI) frente al número de onda normalizado $k$ para $A_{\rho}=0.6, H=1$, $L_{\rho}=L_{u}=0.1,0.2,0.3,0.5, U_{o}=1$. 
(a)

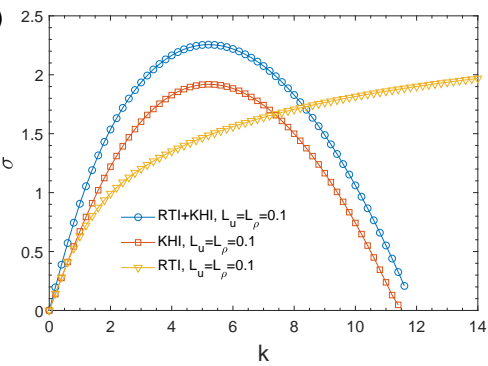

(c)

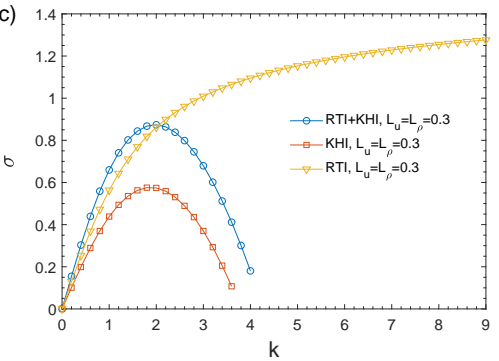

(b)

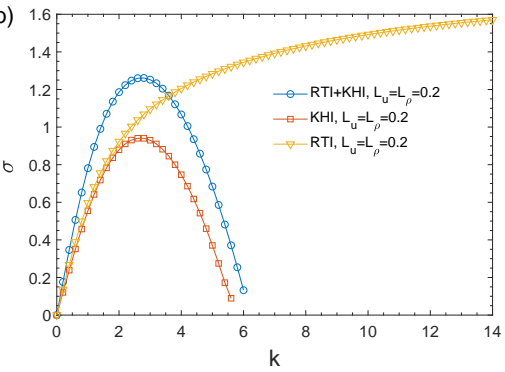

(d)

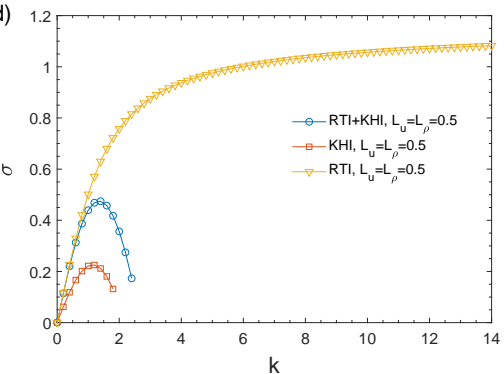

Figura 4.19: Tasa de crecimiento normalizada de la inestabilidad $\sigma$ frente al número de onda normalizado $k$ para $L_{\rho}=L_{u}=0.1,0.2,0.3,0.5, A_{\rho}=0.6$, $H=1, U_{o}=1$. Se representan por separado los casos individuales de RTI y KHI, así como el efecto combinado de las dos inestabilidades. 
Capítulo 4. Inestabilidad de Kelvin-Helmholtz (KHI) en un
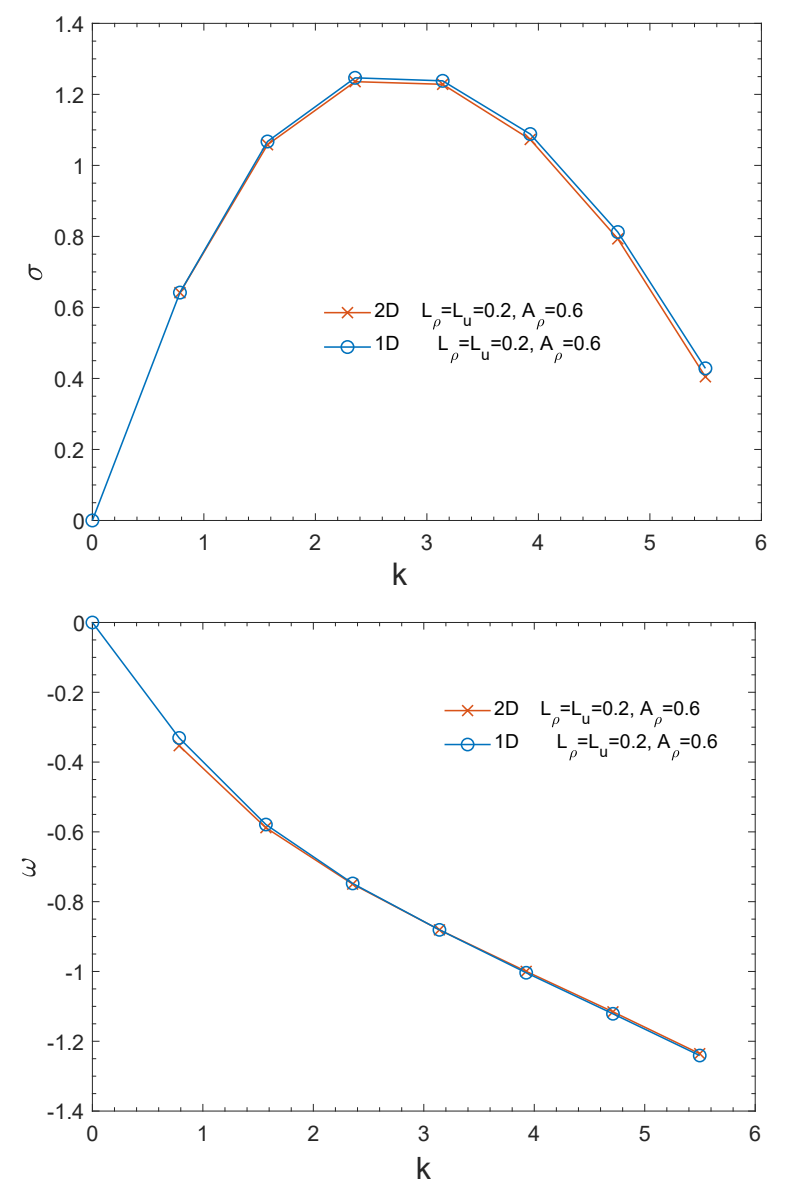

Figura 4.20: Tasa de crecimiento de la inestabilidad (RTI+KHI) $\sigma$ (gráfica superior) y frecuencia $\omega$ (gráfica inferior) frente al número de onda normalizado $k$ para $A_{\rho}=0.6, L_{\rho}=L_{u}=0.2, H=1, U_{o}=1$. 

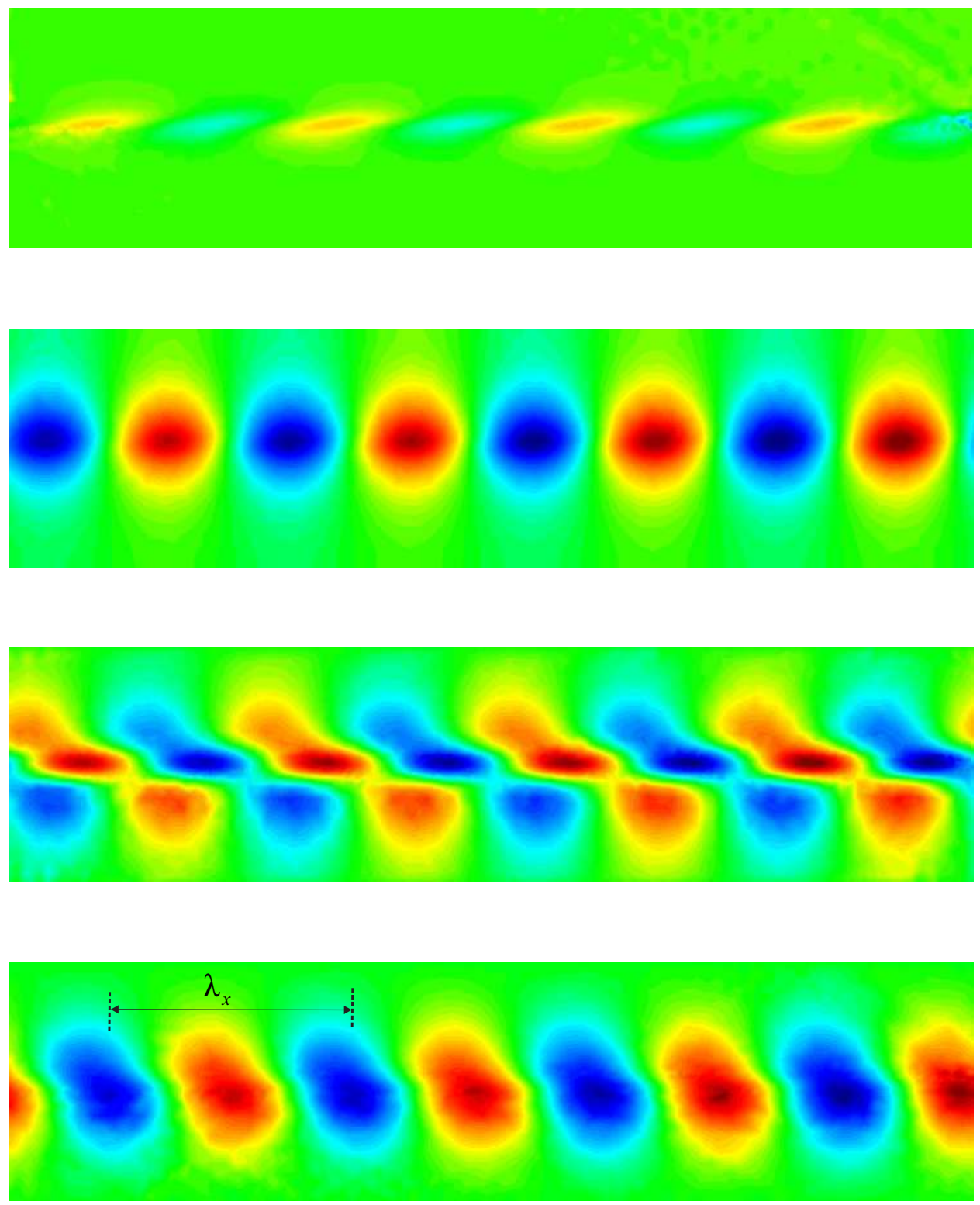

Figura 4.21: Perturbación más inestable para el caso $k=\pi, H=1, L=4$ y $A_{\rho}=0.6(\mathrm{RTI}+\mathrm{KHI})$. Desde la gráfica superior a la inferior, autofunción de densidad, presión, velocidad horizontal y velocidad vertical. Los cálculos fueron llevados a cabo con los parámetros de interfase $L_{\rho}=L_{u}=0.2$. 
Capítulo 4. Inestabilidad de Kelvin-Helmholtz (KHI) en un

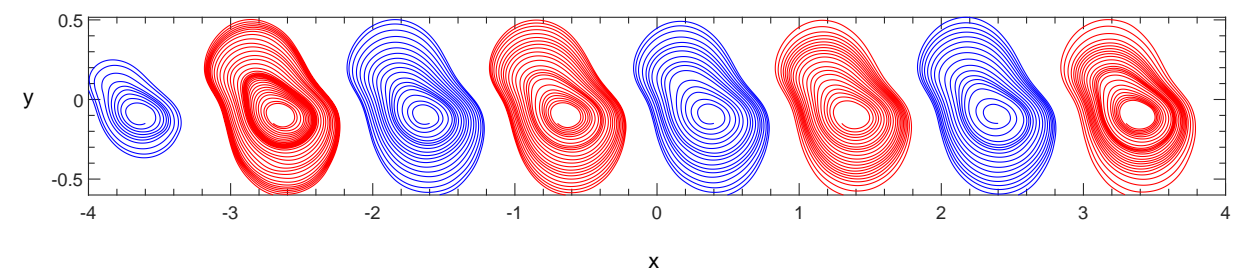

Figura 4.22: Líneas de corriente siendo $L_{\rho}=L_{u}=0.2, H=1, A_{\rho}=0.6$, $U_{o}=1$ y $k_{x}=\pi$, coexistiendo RTI y KHI.

figura 4.21.También es de interés representar las líneas de corriente, relativas a las mencionadas autofunciones (ver figura 4.22). En la figura se observan 8 vórtices: cuatro de ellos con vorticidad positiva (líneas continuas de color rojo) y otros cuatro con vorticidad negativa (líneas continuas de color azul).

\subsection{Conclusiones}

Hemos estudiado numéricamente el comportamiento de la KHI en régimen lineal para un flujo base en densidad y velocidad del tipo tangente hiperbólica [95], 94] de características similares al analizado por otros autores, como Wang et al. 93], 101, Ye et al. [102, y Hoshoudy and H. Cavus [96], que lo habían diseñado mediante funciones exponenciales. El estudio se ha realizado en un dominio finito tanto en una formulación 1D (método espectral) como en 2D (FEM) siendo esta última formulación la que nos ha permitido obtener una representación de las autofunciones del problema. Hemos encontrado que la tasa de crecimiento de la inestabilidad $\sigma$ tiene un crecimiento monótono a medida que aumenta el número de onda $k$ hasta alcanzar un valor máximo $\sigma_{\max }$, para luego decrecer monotonamente con $k$. Este comportamiento difiere del hallado por Wang et al. [101] y Ye et al. [102] mediante un análisis analítico, donde la tasa de crecimiento de la inestabilidad es monótonamente creciente en $k$ hasta alcanzar un valor asintótico máximo. Este hecho nos lleva a concluir que el comportamiento de las relaciones de dispersión de los dos tipos de flujos base difieren, y que no siempre se podrían llevar a cabo validaciones como la realizada por Gan et al. 94.

Por otro lado, hemos analizado, tanto en una formulación 1D y 2D, el problema de estabilidad en el caso de coexistencia de RTI y KHI con tran- 
siciones difusas en densidad y velocidad. En primer lugar, los resultados obtenidos nos permiten verificar lo observado por Ye et al. [102, Wang et al. [101] y Guzdar et al. [100, i.e., que un aumento de $L=L_{u}=L_{\rho}$ da lugar a una reducción del valor de $\sigma$ para un $k$ determinado, manteniendo el resto de parámetros del problema constantes. En segundo lugar, hemos verificado la total inhibición de la inestabilidad, de acuerdo con Guzdar et al. [100], a partir de un número de onda determinado. En tercer lugar, hemos comprobado que el aumento de $L$ lleva aparejado una reducción del intervalo de existencia del espectro de modos inestables, tal y como observaron Guzdar et al. [100]. Por último, habría que añadir que nuestro estudio es más completo y arroja más luz sobre el fenómeno, porque la formulación $2 \mathrm{D}$ nos permite determinar las amplitudes de las perturbaciones y las líneas de corriente en su forma bidimensional. 



\section{CAPÍtulo 5 \\ Análisis BiGlobal de la inestabilidad de vórtices de densidad variable}

En este capítulo, vamos a estudiar la inestabilidad de vórtices de densidad variable mediante un análisis BiGlobal de estabilidad lineal. En primer lugar, nos centraremos en el flujo base de un chorro jet en torbellino de densidad variable modelizado por el Batchelor q-vortex (Bqv), cuya estabilidad fue estudiada con anterioridad por otros autores como Di Pierro y Abid [2] desde el punto de vista de un análisis espacio-temporal. Con un análisis BiGlobal profundizaremos en la investigación llevada a cabo por los referidos autores [2], ya que estos no mostraron en su trabajo las frecuencias de los autovalores ni las autofunciones. Nuestra metodología nos permitirá obtener las mencionadas frecuencias, así como la representación de las autofunciones de la densidad perturbada, lo cual ayuda a una mayor compresión del problema de inestabilidad analizado. En segundo lugar, analizaremos la inestabilidad de Rayleigh-Taylor de un vórtice de Lamb-Ossen de densidad variable, caso estudiado anteriormente por Joly et al. [104], así como por Sipp et al. [103, pero desde el punto de vista de un análisis modal local. Nuestro análisis de inestabilidad BiGlobal nos permitirá obtener las autofunciones de la densidad perturbada que no fueron determinadas por los referidos autores [104, 103] para el vórtice de Lamb-Ossen.

\subsection{Flujo base del problema}

Como flujo base utilizaremos un Bqv cuyo campo de velocidades está definido en (1.8), pero sin flujo externo axial $\left(u_{b}=0\right)$, y se caracterizará por tener densidad variable [2] que vendrá definida por la expresión (1.16).

En el problema que nos ocupa, trabajemos con coordenadas cartesianas. Por ello, las expresiones del flujo base (1.8) en coordenadas cartesianas, que denotaremos por $U, V$ y $W$ para las componentes de la velocidad en $x$, 
Capítulo 5. Análisis BiGlobal de la inestabilidad de vórtices de 112 densidad variable

en $y$ y en $z$, respectivamente, junto con determinadas derivadas de interés, vendrán dadas por

$$
\begin{aligned}
& U=-\frac{u_{c} \Gamma \delta\left(1-\mathrm{e}^{-\frac{x^{2}+y^{2}}{\delta^{2}}}\right) y}{2 \pi\left(x^{2}+y^{2}\right)} \\
& V=\frac{u_{c} \Gamma \delta\left(1-\mathrm{e}^{-\frac{x^{2}+y^{2}}{\delta^{2}}}\right) x}{2 \pi\left(x^{2}+y^{2}\right)} \\
& W=u_{c} e^{-\frac{\left(x^{2}+y^{2}\right)}{\delta^{2}}} \\
& U_{x}=-\frac{\Gamma u_{c} \mathrm{e}^{-\frac{x^{2}+y^{2}}{\delta^{2}}} x y\left[x^{2}+y^{2}-\left(-1+\mathrm{e}^{\frac{x^{2}+y^{2}}{\delta^{2}}}\right) \delta^{2}\right]}{\pi\left(x^{2}+y^{2}\right)^{2} \delta} \\
& U_{y}=\frac{\left(1-\mathrm{e}^{-\frac{x^{2}+y^{2}}{\delta^{2}}}\right) y^{2} \Gamma u_{c} \delta}{\pi\left(x^{2}+y^{2}\right)^{2}}-\frac{\left(1-\mathrm{e}^{-\frac{x^{2}+y^{2}}{\delta^{2}}}\right) \Gamma u_{c} \delta}{2 \pi\left(x^{2}+y^{2}\right)}-\frac{\mathrm{e}^{-\frac{x^{2}+y^{2}}{\delta^{2}}} y^{2} \Gamma u_{c}}{\pi\left(x^{2}+y^{2}\right) \delta} \\
& V_{y}=\frac{u_{c} \mathrm{e}^{-\frac{x^{2}+y^{2}}{\delta^{2}}} x y \Gamma\left[x^{2}+y^{2}-\left(-1+\mathrm{e}^{\frac{x^{2}+y^{2}}{\delta^{2}}}\right) \delta^{2}\right]}{\pi\left(x^{2}+y^{2}\right)^{2} \delta} \\
& V_{x}=-\frac{\left(1-\mathrm{e}^{-\frac{x^{2}+y^{2}}{\delta^{2}}}\right) x^{2} \Gamma \delta u_{c}}{\pi\left(x^{2}+y^{2}\right)^{2}}+\frac{\left(1-\mathrm{e}^{-\frac{x^{2}+y^{2}}{\delta^{2}}}\right) \Gamma \delta u_{c}}{2 \pi\left(x^{2}+y^{2}\right)}+\frac{\mathrm{e}^{-\frac{x^{2}+y^{2}}{\delta^{2}}} x^{2} \Gamma u_{c}}{\pi\left(x^{2}+y^{2}\right) \delta} \\
& W_{x}=-2 x u_{c} e^{-\frac{\left(x^{2}+y^{2}\right)}{\delta^{2}}} \\
& W_{y}=-2 y u_{c} e^{-\frac{\left(x^{2}+y^{2}\right)}{\delta^{2}}}
\end{aligned}
$$

Las expresiones del flujo base en velocidad (5.1)-(5.9) las normalizaremos con respecto a la velocidad característica $u_{c} \mathrm{y}$ una longitud característica $l_{c}$ que será el radio del vórtice $\delta$. Con respecto al flujo base en densidad (1.16), convertiremos la expresión en adimensional dividiendo dicha expresión por la densidad característica $\rho_{c}$ que tomaremos como $\rho_{b}$, la densidad en los alrededores del vórtice. 
Tabla 5.1: Validación de los resultados obtenidos con los de Fabre et al. 3].

\begin{tabular}{cccccc}
\hline \hline$m$ & $R e$ & $q$ & $k_{z}$ & $\sigma$ (Fabre et al. $[3]$ ) & $\sigma$ (presente trabajo) \\
\hline 3 & $10^{2}$ & 0.656 & 1.582 & 0.1431 & 0.14305 \\
3 & $10^{3}$ & 0.761 & 1.659 & 0.3245 & 0.32446 \\
2 & $10^{3}$ & 0.680 & 1.174 & 0.2956 & 0.29565 \\
3 & $10^{4}$ & 0.776 & 1.664 & 0.3514 & 0.35135 \\
2 & $10^{4}$ & 0.691 & 1.180 & 0.3119 & 0.31189 \\
\hline
\end{tabular}

\subsection{Resolución numérica de las LNSE mediante una malla $2 \mathrm{D}$}

Con objeto de resolver las LNSE, reduciremos nuestro problema a un problema de autovalores generalizado que se corresponden al sistema (2.66). Nos centraremos en el caso particular en el que despreciaremos el efecto de la gravedad. La discretización de las LNSE será mediante elementos finitos, utilizando elementos tipo Taylor-Hood P2-P1. Utilizaremos un dominio computacional 2D con condiciones de contorno homogéneas tipo Dirichlet $\hat{v}=0$. La resolución numérica se llevará a cabo mediante el método de Arnoldi, usando un subespacio de Krylov de 10 vectores. En la figura 5.1 se muestra la malla utilizada que tiene 82629 nodos y 22102 elementos.

\subsubsection{Bqv de densidad constante. Validación de la formula- ción $2 \mathrm{D}$}

En primer lugar, validaremos los resultados obtenidos con nuestro código con los de Fabre et al. 3] para el caso de un vórtice aislado de densidad constante $R=1$ tipo Bqv. En sus cálculos estos autores utilizaron un radio del núcleo del vórtice y del jet $\delta=1$. En la tabla adjunta 5.1 se establece una comparación entre la tasa de crecimiento de la inestabilidad calculada con nuestro código (ver columna sexta) y la obtenida por dichos autores (columna quinta) para diferentes $R e, q, k_{z}$ y $m$, obteniéndose un buen acuerdo.

En segundo lugar, llevaremos a cabo una segunda validación, reproduciendo los autovalores obtenidos por Delbende et al. 4], también para un Bqv de densidad constante, pero para un número de Reynolds fijo $R e=667$, $\delta=1$ y $q=0.8$. En la tabla 5.2 se pueden comparar los valores numéricos de las tasas de crecimiento de la inestabilidad y las frecuencias obtenidas por este autor [4], con las obtenidas para diferentes números de onda axial 
Capítulo 5. Análisis BiGlobal de la inestabilidad de vórtices de 114 densidad variable
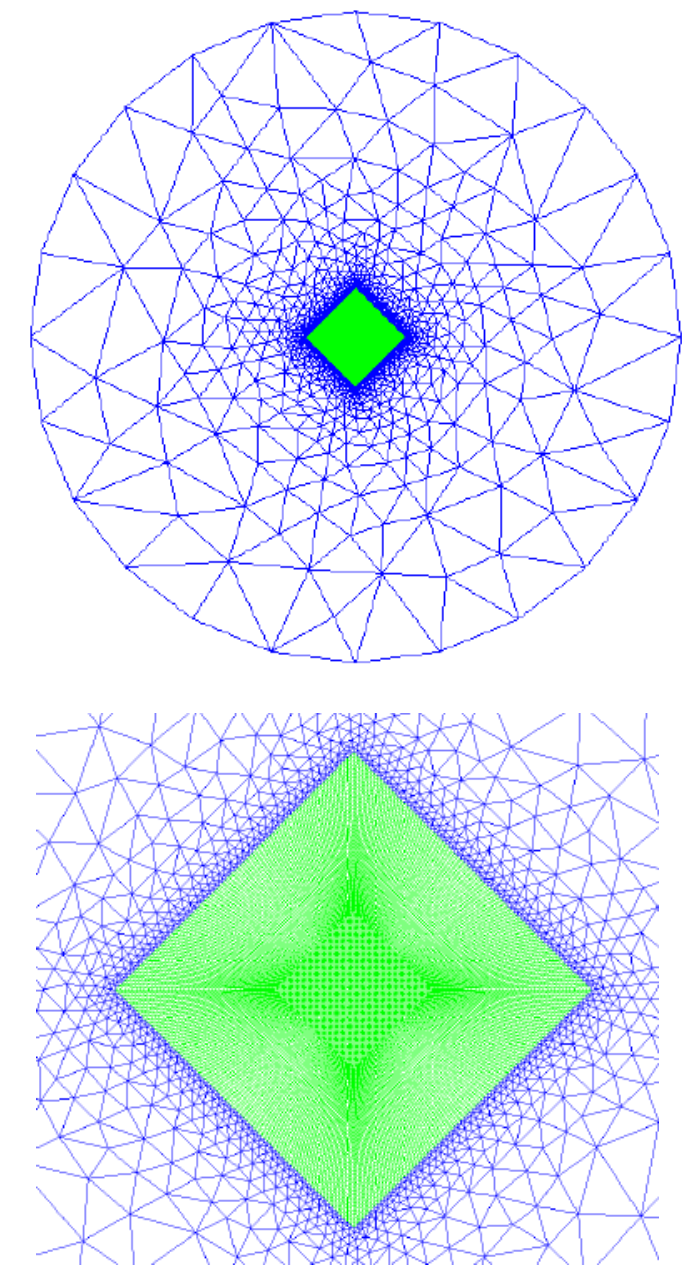

Figura 5.1: Malla para el análisis BiGlobal de la inestabilidad del Bqv aislado (imagen superior). También se muestra en detalle la parte central ampliada de la malla para su claridad (imagen inferior). 


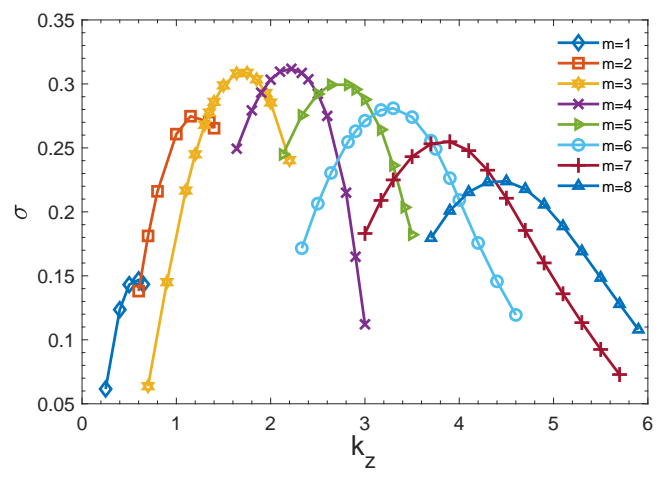

Figura 5.2: Tasa de crecimiento de la inestabilidad $\sigma$ frente al número de onda axial $k_{z}$ para $R e=667, q=0.8$ y Bqv de densidad constante.

$k_{z}$ y azimutal $m$ mediante el presente método (ver columnas cuarta y sexta de la tabla). Se puede observar que los resultados que hemos hallado son muy similares a los de Delbende et al. [4, obteniéndose también un buen acuerdo.

Tabla 5.2: Validación de los resultados obtenidos con los de Delbende et al. [4] para $R e=667, \delta=1$ y $q=0.8$.

\begin{tabular}{cccccc}
\hline \hline$m$ & $k_{z}$ & $\sigma$ (Delbende et al. $)$ & $\sigma$ & $\omega$ (Delbende et al. $)$ & $\omega$ \\
\hline 2 & 1.4 & 0.2672 & 0.2654 & -0.2075 & -0.2332 \\
3 & 1.85 & 0.3029 & 0.3036 & -0.6775 & -0.6436 \\
4 & 2.4 & 0.3011 & 0.3037 & -0.9754 & -0.9757 \\
5 & 2.64 & 0.2994 & 0.3020 & -1.4304 & -1.4550 \\
6 & 3.5 & 0.2715 & 0.2741 & -1.6041 & -1.6210 \\
7 & 3.9 & 0.2563 & 0.2548 & -2.0108 & -2.0020 \\
\hline
\end{tabular}

Por otro lado, con objeto de reproducir la figura 5.a del trabajo de Delbende et al. [4], en la gráfica de la figura 5.2 se ha representado la tasa de crecimiento de la inestabilidad $\sigma$ frente al número de onda axial $k_{z}$. Asimismo, en la gráfica de la figura 5.3 se ha reproducido la gráfica 5 .b del referido trabajo donde se representa la frecuencia $\omega$ frente a $k_{z}$ para diferentes números de onda azimutal $m$.

Por último, hemos llevado a cabo otra validación con los resultados hallados por Billant et al. [27] para un Bqv de densidad constante $R=1$ 


\section{Capítulo 5. Análisis BiGlobal de la inestabilidad de vórtices de 116 densidad variable}

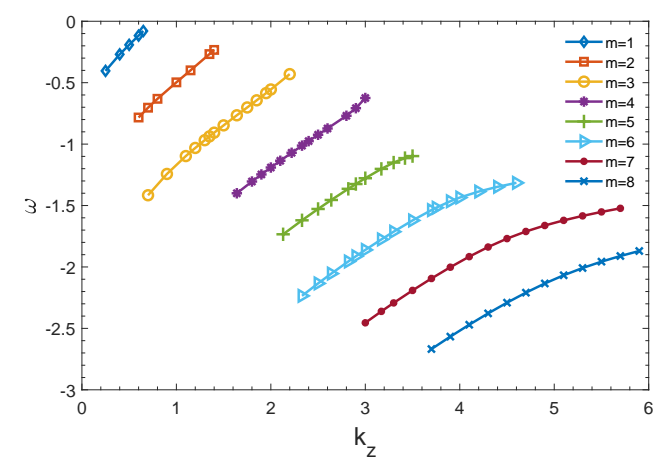

Figura 5.3: Frecuencia $\omega$ frente al número de onda axial $k_{z}$ para $R e=667$, $q=0.8$ y Bqv de densidad constante.

en ausencia de viscosidad $(R e \rightarrow \infty)$. Los parámetros del Bqv son $\delta=1$ y $q=0.8$. Asimismo, hemos intentado reproducir la figura 1.b de [27] en la que se representa la tasa de crecimiento de la inestabilidad $\sigma$ frente al número de onda $k_{z}$ siendo $m=8$. En la figura 5.4 se pueden observar tres resultados: los obtenidos con el FEM en la formulación 2D, los de Billant et al. 27] y los obtenidos mediante la fórmula asintótica (18) de Di Pierro et al. [105] obteniéndose un buen acuerdo. Asimismo, se ha representado la frecuencia $\omega$ frente a $k_{z}$ para $\delta=1$ y $q=0.8$, comparando también los resultados del FEM con los de [105] y [27]: estos resultados se pueden considerar aceptables.

\subsubsection{Bqv de densidad variable}

A continuación, analizaremos la inestabilidad de un Bqv de densidad variable que vendrá dada por la forma adimensional de la expresión 1.16

$$
R=1+(s-1) e^{-\epsilon^{2} \bar{r}^{2}}
$$

donde $\bar{r}=r / \delta$, siendo $\delta$ el radio característico del perfil de velocidad, $\epsilon=$ $\delta / \delta_{\rho}, \delta_{\rho}$ el radio característico del perfil de densidad y $s=\rho_{a} / \rho_{b}$ el cociente de densidades. A partir de este momento y por simplicidad, prescindiremos de las tildes para denotar las magnitudes adimensionales.

Analizaremos el caso de Di Pierro et al. [2] para $s=2, \delta=\delta_{\rho}=1$ $(\epsilon=1), R e=667$ y $q=0.8$. Para concretar la validez del modelo $2 \mathrm{D}$, vamos a realizar un análisis de convergencia. Para ello, utilizaremos tres mallas diferentes: malla 1 (82629 nodos y 22102 elementos), malla 2 (67989 nodos 
5.2. Resolución numérica de las LNSE mediante una malla $2 \mathrm{D} 17$
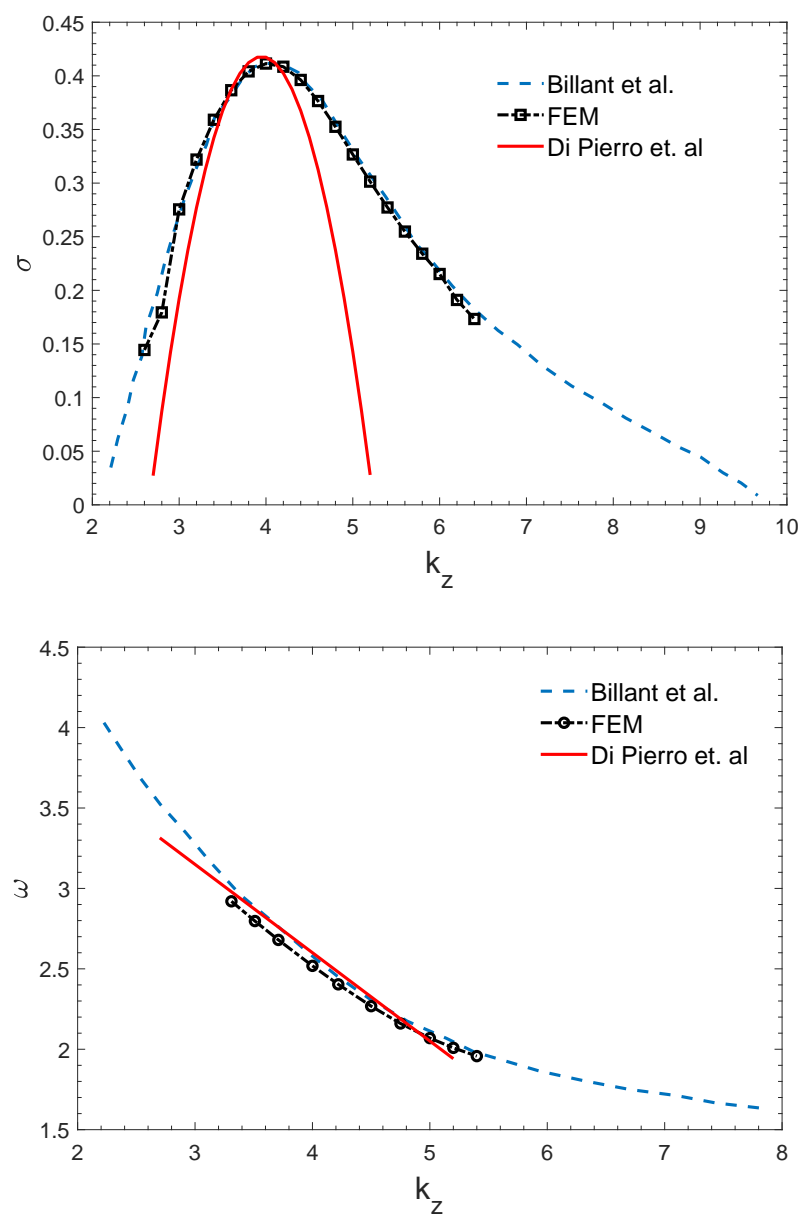

Figura 5.4: Tasa de crecimiento de la inestabilidad $\sigma$ (grafica superior) y frecuencia $\omega$ (gráfica inferior) frente al número de onda axial $k_{z}$ para un Bqv de densidad constante siendo $q=0.8$ y $R e \rightarrow \infty$. 
Capítulo 5. Análisis BiGlobal de la inestabilidad de vórtices de 118 densidad variable

y 17982 elementos) y malla 3 (19993 nodos y 5484 elementos). Los resultados se muestran en la tabla 5.3 .

Tabla 5.3: Análisis de convergencia para $\delta=\delta_{\rho}=1(\epsilon=1), R e=667 \mathrm{y}$ $q=0.8$.

\begin{tabular}{cccccc}
\hline \hline$k_{z}$ & $m$ & $\sigma$ & $\omega$ & Nodos & Elementos \\
\hline 1.70 & 3 & 0.38102788 & 0.68035913 & 19993 & 5484 \\
1.70 & 3 & 0.38103183 & 0.68035530 & 67989 & 17982 \\
1.70 & 3 & 0.38103192 & 0.68035531 & 82629 & 22102 \\
\hline 2.24 & 4 & 0.39932391 & 1.01786839 & 19993 & 5484 \\
2.24 & 4 & 0.39932900 & 1.01786069 & 67989 & 17982 \\
2.24 & 4 & 0.39949794 & 1.02360433 & 82629 & 22102 \\
\hline 3.21 & 6 & 0.39682503 & 1.73064343 & 19993 & 5484 \\
3.21 & 6 & 0.39684191 & 1.73062028 & 67989 & 17982 \\
3.21 & 6 & 0.39684220 & 1.73062012 & 82629 & 22102 \\
\hline 3.50 & 7 & 0.38004427 & 2.18300398 & 19993 & 5484 \\
3.50 & 7 & 0.38008749 & 2.18296545 & 67989 & 17982 \\
3.50 & 7 & 0.38008819 & 2.18296527 & 82629 & 22102 \\
\hline 3.75 & 7 & 0.38504263 & 2.05055762 & 19993 & 5484 \\
3.75 & 7 & 0.38506978 & 2.05051771 & 67989 & 17982 \\
3.75 & 7 & 0.38507028 & 2.05051741 & 82629 & 22102 \\
\hline 4.00 & 8 & 0.36386311 & 2.51868416 & 19993 & 5484 \\
4.00 & 8 & 0.36393062 & 2.51861661 & 67989 & 17982 \\
4.00 & 8 & 0.36393179 & 2.51861611 & 82629 & 22102 \\
\hline 5.00 & 8 & 0.30886016 & 2.06929637 & 19993 & 5484 \\
5.00 & 8 & 0.30888937 & 2.06925036 & 67989 & 17982 \\
5.00 & 8 & 0.30889020 & 2.06925006 & 82629 & 22102 \\
\hline
\end{tabular}

Por otro, lado en las gráficas de la figura 5.5 se han representado la tasa de crecimiento de la inestabilidad $\sigma$ y la frecuencia $\omega$ frente al número de onda axial $k_{z}$, siendo los parámetros del Bqv de densidad variable $R e=667$, $q=0.8, \delta=\delta_{\rho}=1$ y $s=2$. 
5.2. Resolución numérica de las LNSE mediante una malla 2 D19
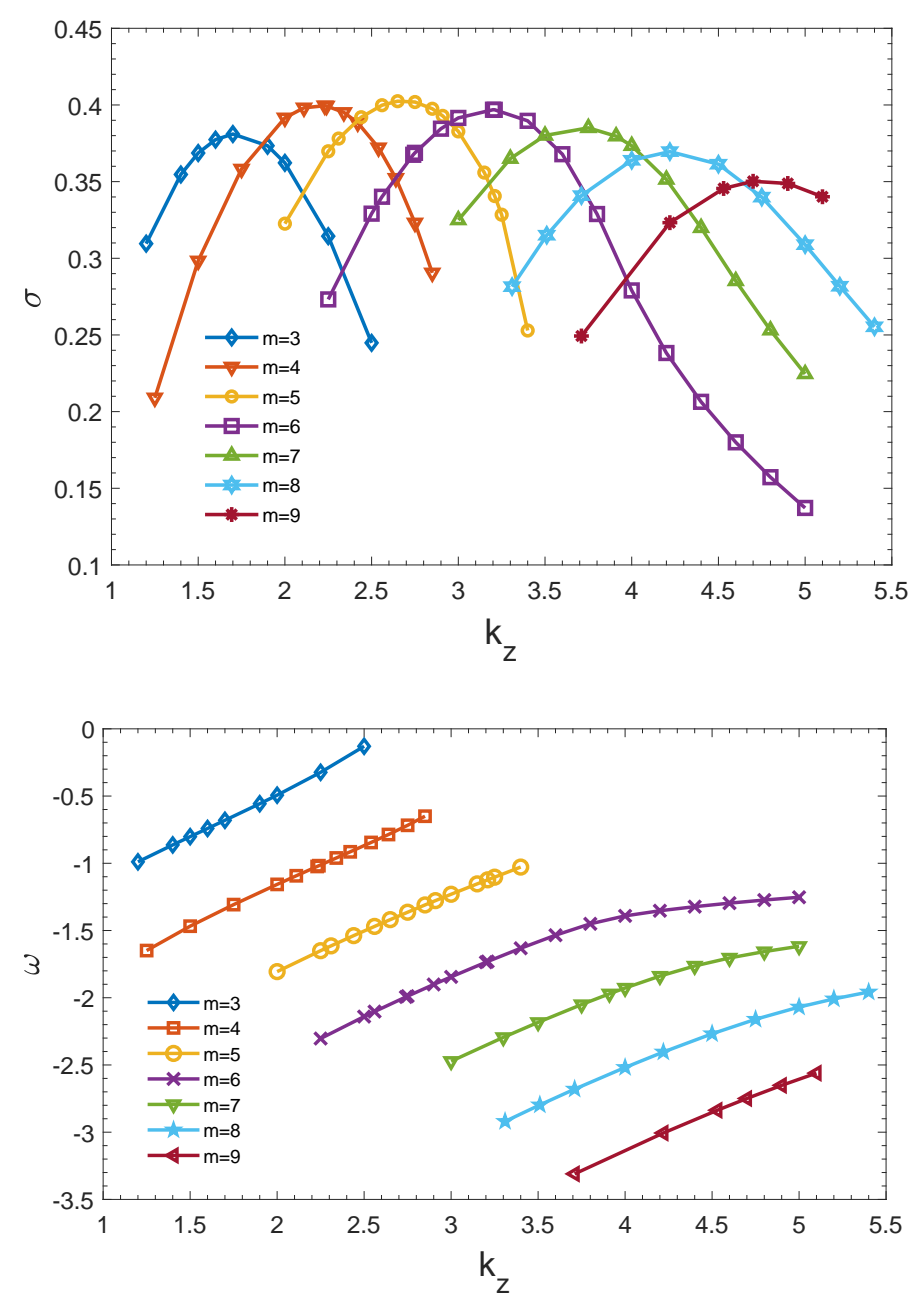

Figura 5.5: Tasa de crecimiento de la inestabilidad $\sigma$ (grafica superior) y frecuencia $\omega$ (gráfica inferior) frente al número de onda axial $k_{z}$ para un Bqv de densidad variable con $s=2, R e=667, q=0.8$ y $\delta=\delta_{\rho}=1$.

En la gráfica 5.6 se ha establecido una comparativa entre las tasas de crecimiento de la inestabilidad $\sigma$ de los modos $m=3$ y $m=4$, correspondientes a los cálculos de Di Pierro [2] para $s=2, R e=667$ y $q=0.8$ en su formulación 1D, así como los obtenidos para los mismos valores en la formulación 2D llevada a cabo en el presente trabajo. Se puede comprobar que los máximos de las tasas de crecimiento de la inestabilidad $\sigma$ se 


\section{Capítulo 5. Análisis BiGlobal de la inestabilidad de vórtices de 120 densidad variable}

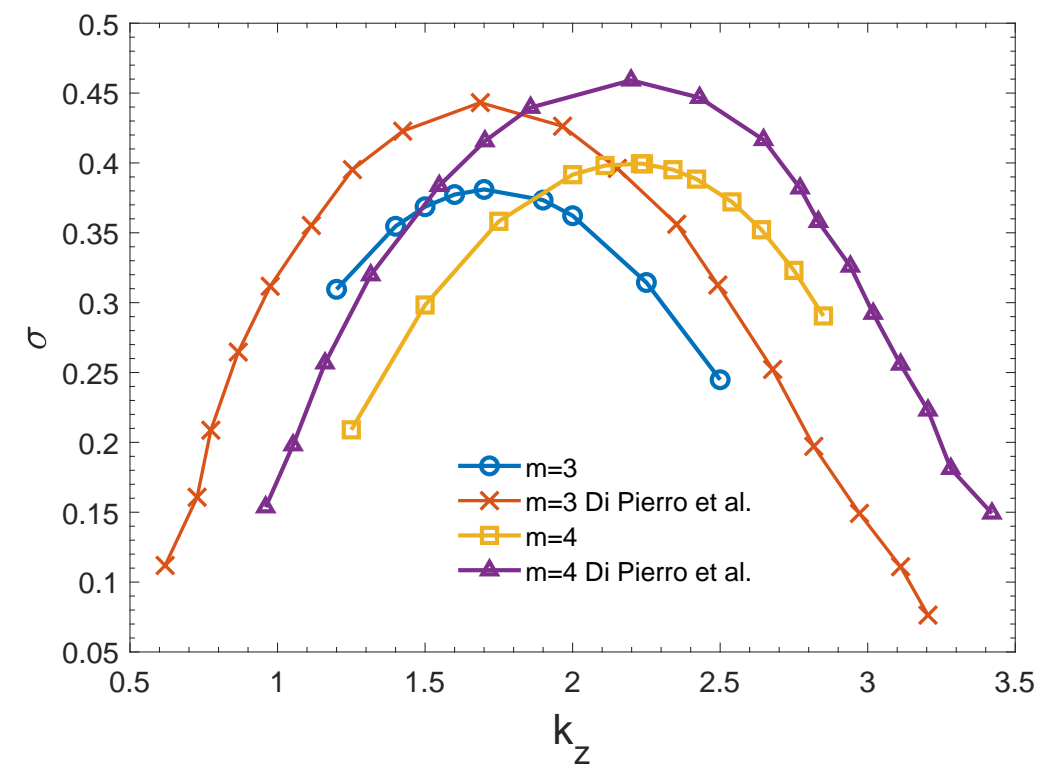

Figura 5.6: Comparativa de $\sigma$ frente a $k_{z}$ para $m=3$ y $m=4$ siendo $R e=667, q=0.8, s=2$ y $\delta=\delta_{\rho}=1$. Se han representado los resultados obtenidos en este trabajo junto con los de Di Pierro et al. [2].

alcanzan, aproximadamente, para el mismo número de onda. Así, para el modo $m=3$ los máximos se alcanzan en $k_{z} \simeq 1.7$, tomando los valores de $\sigma_{\max }=0.3810$ y $\sigma_{\max }=0.4432$, para las formulaciones $2 \mathrm{D}$ y $1 \mathrm{D}$, respectivamente (la discrepancia es del 14\%); para el modo $m=4$ los máximos se observan en $k_{z} \simeq 2.23$, siendo $\sigma_{\max }=0.3995$ y $\sigma_{\max }=0.4591$, para las formulaciones 2D y 1D, respectivamente (la discrepancia es del 13\%). Los máximos de $\sigma$ para el resto de modos con números de onda azimutales $m=5,6,7 \ldots$ (no representados) siguen un comportamiento similar con respecto a los resultados obtenidos por Di Pierro et al. [2]. De estos resultados se deduce que los modos obtenidos en la formulación $2 \mathrm{D}$ son más estables que los correspondientes a la formulación 1D.

En las figuras 5.7 y 5.8 se han representado algunas autofunciones de la densidad perturbada. Así, en la figura 5.7 se observa el modo con $m=3$ y $k_{z}=2.25$ (parte derecha) en el que se pueden apreciar claramente tres lóbulos de color rojo (donde la amplitud es positiva) y tres de color azul (donde la amplitud es negativa); asimismo, en la parte derecha se aprecia con cuatro lóbulos de amplitud positiva y cuatro de amplitud negativa el 


\subsection{Resolución numérica de las LNSE mediante una malla 2 D21}

modo $m=4$ y $k_{z}=2$. Por otro lado, en la figura 5.8 se han representado las amplitudes de la densidad perturbada para $m=5, k_{z}=3$ (gráfica izquierda) y $m=7, k_{z}=4.6$ (gráfica derecha).
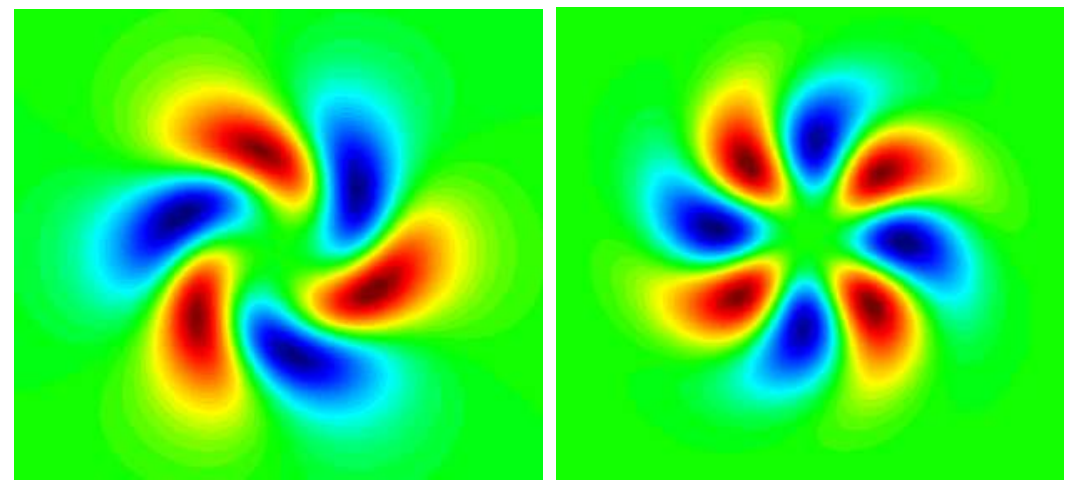

Figura 5.7: Amplitud de la densidad perturbada para $R e=667, q=0.8$, $s=2, \delta=\delta_{\rho}=1$. En la gráfica izquierda se muestra la autofunción de la densidad perturbada para $m=3$ y $k_{z}=2.25$. En la gráfica derecha, para $m=4$ y $k_{z}=2.0$.
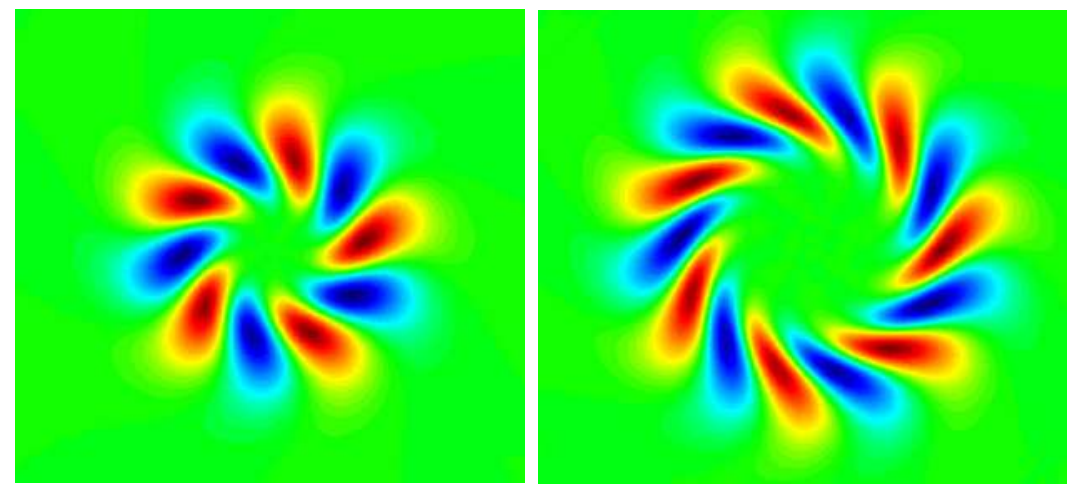

Figura 5.8: Amplitud de la densidad perturbada para $R e=667, q=0.8$, $s=2, \delta=\delta_{\rho}=1$. En la gráfica izquierda se muestra la autofunción de la densidad para $m=5$ y $k_{z}=3$. En la gráfica derecha, para $m=7$ y $k_{z}=4.6$. 
Capítulo 5. Análisis BiGlobal de la inestabilidad de vórtices de 122 densidad variable

\subsubsection{Vórtice de Lamb-Oseen de densidad variable}

Como mencionamos en su momento, el campo de velocidades que caracteriza a un vórtice de Lamb-Oseen lo podemos obtener eliminando la componente axial del campo de velocidades $\left(u_{z}=0\right)$ del Bqv en la expresión (1.8). La densidad variable del vórtice vendrá dada por la expresión (1.16) del Bqv.

En la gráfica superior de la figura 5.9 se ha representado la tasa de crecimiento de la inestabilidad $\sigma$ frente al número de onda azimutal $m$, siendo los parámetros del vórtice de Lamb-Oseen de densidad variable $q=$ $0.5, \epsilon=6.31, \delta=1, s=3$ y $R e \rightarrow \infty$. En esta gráfica se establece una comparativa entre los resultados numéricos obtenidos por Joly et al. 104, los calculados utilizando la expresión asintótica etiquetada como (22) en el trabajo de Di Pierro et al. [105] y los numéricos obtenidos mediante el FEM en la formulación 2D. En la gráfica de $\sigma$ frente a $k_{z}$ podemos comprobar que, a medida que aumenta el número azimutal $m$, los resultados de Joly et al. [104] se van aproximando a los asintóticos de Di Pierro et al. [105], lo cual era de esperar, puesto que la mencionada expresión asintótica (22) se ha obtenido para $m>>1$. Además, se puede observar que en los tres procedimientos de obtención de $\sigma$ frente a $k_{z}$, el valor máximo de la tasa de crecimiento de la inestabilidad se alcanza en $m=6$. Por otro lado, se puede comprobar que los valores de la tasa de crecimiento de la inestabilidad $\sigma$ obtenidos por el FEM son inferiores a los de [104] y [105], lo cual está en consonancia con los resultados encontrados para el Bqv en la figura 5.6 donde las tasas de crecimiento de la inestabilidad halladas en la formulación 2D eran inferiores a las obtenidas en la formulación 1D [2].

En otro orden de cosas, en la gráfica inferior de la figura 5.9 solo se muestran las frecuencias halladas a través de la expresión asintótica (22) de Di Pierro et al. [105] y las numéricas obtenidas mediante el FEM (formulación 2D), no apareciendo las frecuencias correspondientes al trabajo de Joly et al. [104 porque estos autores solo muestran frecuencias para $\epsilon=2$. Los frecuencias calculadas con el FEM están en buen acuerdo con las de la expresión asintótica de Di Pierro et al. [105] y tienden al valor de éstas a medida que aumenta $m$, como era de esperar.

Para estudiar la validez de los autovalores representados en la figura 5.9 . realizaremos un análisis de convergencia. Para este fin, vamos a utilizar tres mallas diferentes: malla 1 (82629 nodos y 22102 elementos), malla 2 (67989 nodos y 17982 elementos) y malla 3 (19993 nodos y 5484 elementos). Los resultados se muestran en la tabla 5.4 para los modos $m=4,5,6$. 
Tabla 5.4: Análisis convergencia para $R e \rightarrow \infty, q=0.5, \epsilon=6.31, \delta=1 \mathrm{y}$ $s=3$

\begin{tabular}{ccccc}
\hline \hline$m$ & $\sigma$ & $\omega$ & Nodos & Elementos \\
\hline 4 & 0.25538164 & -1.88425074 & 19993 & 5484 \\
4 & 0.25575000 & -1.88170817 & 67989 & 17982 \\
4 & 0.25575417 & -1.88165758 & 82629 & 22102 \\
\hline 5 & 0.265240386 & -2.3896921 & 19993 & 5484 \\
5 & 0.266644740 & -2.3853798 & 67989 & 17982 \\
5 & 0.266668931 & -2.3852939 & 82629 & 22102 \\
\hline 6 & 0.269841498 & -2.8927985 & 19993 & 5484 \\
6 & 0.273125159 & -2.8859902 & 67989 & 17982 \\
6 & 0.273187314 & -2.8858468 & 82629 & 22102 \\
\hline
\end{tabular}

En las figuras 5.10 y 5.11 se han representado algunas autofunciones de la densidad perturbada. Así, en la figura 5.10 se observa el modo con $m=2$ y $k_{z}=0$ (parte derecha) en la que se pueden apreciar claramente dos lóbulos de color rojo (donde la amplitud es positiva) y dos de color azul (amplitud negativa). Por otro lado, en la parte derecha se aprecia, con tres lóbulos de amplitud positiva y tres de amplitud negativa, el modo $m=3$ y $k_{z}=0$. Por otro lado, en la figura 5.11 se han representado las amplitudes de la densidad perturbada para $m=4, k_{z}=0$ (gráfica izquierda) y $m=5, k_{z}=0$ (gráfica derecha).

\subsection{Conclusiones}

En el presente capítulo hemos llevado a cabo un estudio completo de la inestabilidad de un chorro (jet) en remolino de densidad variable, modelizado mediante un Bqv, y la de un vórtice de Lamb-Oseen todo ello desde un enfoque BiGlobal. La inestabilidad de un chorro en remolino de densidad variable con $R e=667$ fue estudiada con anterioridad por Di Pierro y Abid [2], pero en un formulación 1D. Estos autores no obtuvieron las frecuencias de los autovalores, ni las autofunciones. En primer lugar, se ha realizado un estudio de validación reproduciendo los resultados obtenidos por otros autores como Delbende et al. [4], Fabre et al. [3], Billant et al. [27] para un Bqv de densidad constante, obteniéndose un buen acuerdo. En segundo lugar, se han obtenido las autofunciones y autovalores del Bqv de densidad variable. En lo que a autovalores se refiere, se han obtenido las frecuencias que no se hallaron en el mencionado trabajo [2] y se comprueba que las 
Capítulo 5. Análisis BiGlobal de la inestabilidad de vórtices de 124 densidad variable
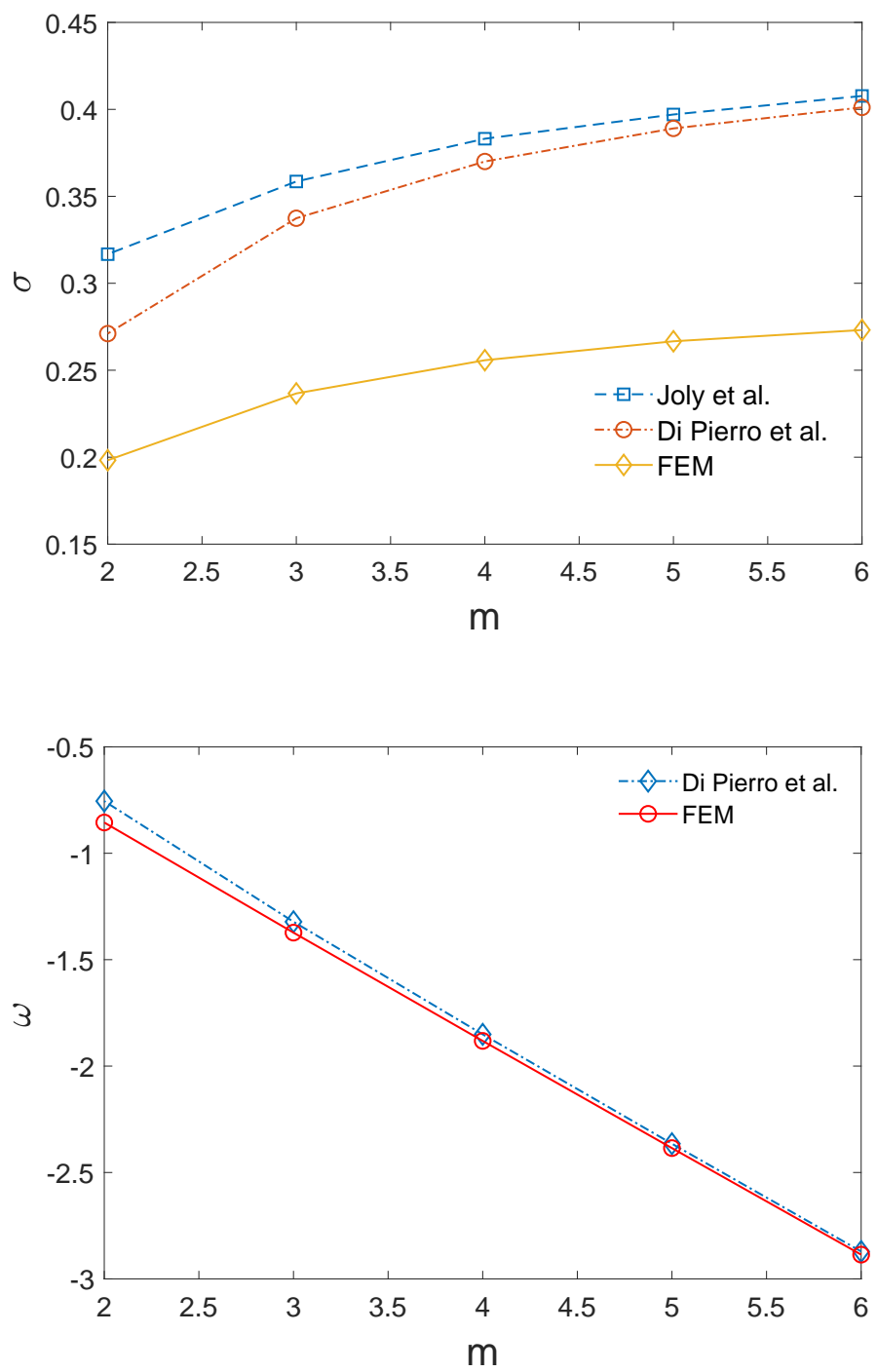

Figura 5.9: Tasa de crecimiento de la inestabilidad $\sigma$ (grafica superior) y frecuencia $\omega$ (gráfica inferior) frente al número de onda azimutal $m$ para $R e \rightarrow \infty, q=0.5, \epsilon=6.31, \delta=1$ y $s=3$. 

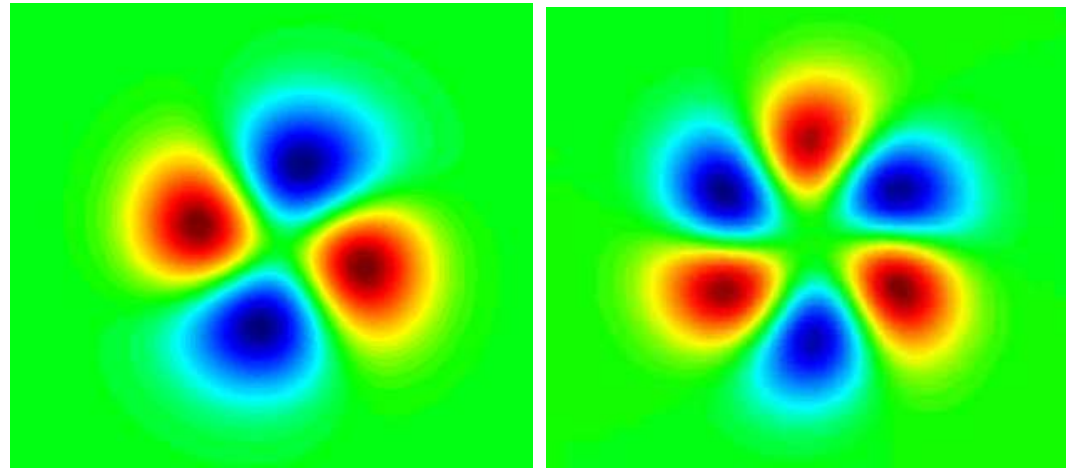

Figura 5.10: Amplitud de la densidad perturbada para $R e \rightarrow \infty, q=0.5$, $s=3, \epsilon=6.31, \delta=1$ y $k_{z}=0$. En la gráfica izquierda se muestra la autofunción de la densidad perturbada para $m=2$. En la gráfica derecha, para $m=3$.
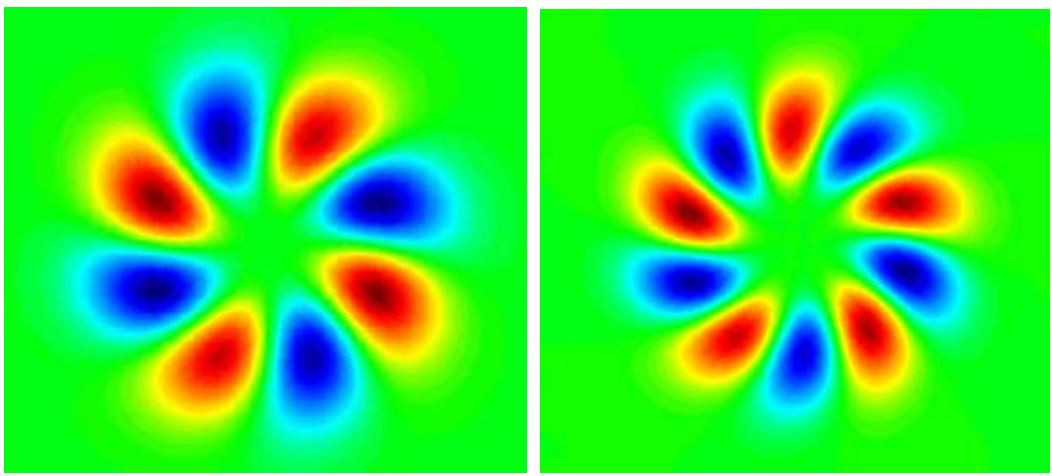

Figura 5.11: Amplitud de la densidad perturbada para $R e \rightarrow \infty, q=0.5$, $s=3, \epsilon=6.31, \delta=1$ y $k_{z}=0$. En la gráfica izquierda se muestra la autofunción de la densidad perturbada para $m=4$. En la gráfica derecha, para $m=5$. 
Capítulo 5. Análisis BiGlobal de la inestabilidad de vórtices de 126 densidad variable

tasas de crecimiento de la inestabilidad $\sigma$ son inferiores a las obtenidas por Di Pierro y Abid [2]: i.e. los modos obtenidos parecen ser más estables. Con respecto a las autofunciones, se ha representado la densidad perturbada para diferentes números de onda azimutal $m$ y axial $k_{z}$. Con respecto al vórtice de Lamb-Oseen de densidad variable, se ha estudiado la RTI en el límite de $R e \rightarrow \infty$, obteniendo los autovalores y comparándolos con los obtenidos por Joly et al. [104. Como novedad, se han obtenido las autofunciones de la densidad perturbada en función del número de onda azimutal que no habían sido estudiadas en el trabajo de estos autores. 
CAPÍtulo 6

\section{El continuum en la RTI con tensión superficial}

En este capítulo nos centraremos en investigar la RTI en la superficie libre de separación de dos fluidos no viscosos, incompresibles y semi-infinitos: uno cuya densidad crece exponencialmente y que está soportado por otro de densidad constante, en presencia de un campo gravitatorio. Es útil plantear el problema de la estabilidad del sistema de los dos fluidos desde el punto de vista de un problema de valor inicial (IVP), de esta forma nos aseguramos la inclusión de ciertos modos que pertenecen a un espectro continuo que sería obviado de estudiar el problema de estabilidad desde un análisis en modos normales. Esta metodología incluye el análisis de la perturbación en el plano complejo, teniendo ésta un corte de ramificación asociado al espectro continuo de modos RTI internos y dos polos asociados a los modos superficiales (espectro discreto). Además, encontramos un nuevo rol para la tensión superficial: transformar los modos del espectro discreto en modos del espectro continuo para un número de onda crítico. Como consecuencia, no existe un número de onda de corte para el cual la tasa de crecimiento de la inestabilidad sea cero, como se había pensado hasta la fecha. Finalmente, encontramos que cuando solo existe el espectro continuo el crecimiento de la perturbación, con respecto del tiempo, es más lento que el puramente exponencial.

\subsection{Planeamiento del problema. Análisis lineal de estabilidad}

Consideremos un sistema similar al usado en la referencia [67], donde un fluido vertical estratificado es soportado por un fluido incompresible de densidad constante, encontrándose en reposo en un sistema de coordenadas $x, y, z$ en la presencia de un campo gravitatorio de intensidad $\mathbf{g}=(0,-g, 0)$. Ejemplos típicos de fluidos estratificados los tenemos en la Tierra, en el océano y en la atmósfera. En el estado no perturbado, vamos a considerar 
128 Capítulo 6. El continuum en la RTI con tensión superficial

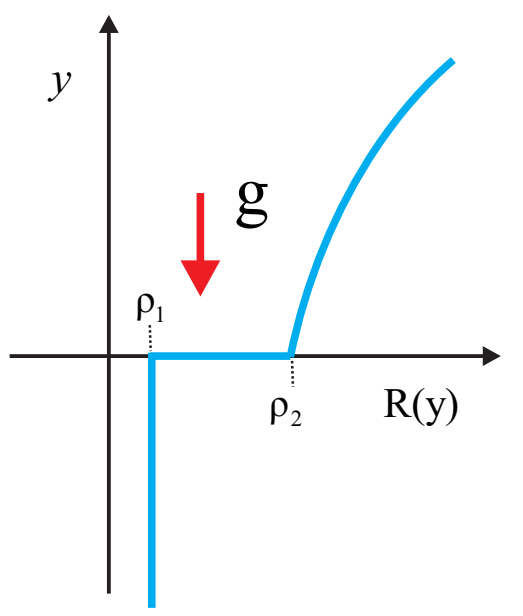

Figura 6.1: Perfil de densidad en el estado no perturbado.

que ambos fluidos que forman el sistema se hallan en reposo i.e., $\mathbf{U}=0$. En este estado la interfase que separa los dos fluidos en $y=y_{o}=0$ es una superficie libre.

Además, supondremos que los fluidos son semi-infinitos. La distribución de densidad $R(y)$ viene dada por la ecuación (6.1) siendo representada en la figura 6.1

$$
R(y) \equiv \begin{cases}\rho_{2} e^{\beta y} & y>0 \\ \rho_{1} & y<0\end{cases}
$$

donde $\rho_{1}$ y $\rho_{2}$ son constantes independientes, siendo $\rho_{2}>\rho_{1}$, representando $\beta$ la inversa de la longitud característica de la densidad en su correspondiente región.

En el equilibrio, y puesto que el campo g es uniforme, la densidad $R$ y la presión del fluido $P$, que son funciones de la coordenada $y$, estarían relacionadas entre sí mediante la siguiente ecuación que no es más que la ecuación hidrostática del equilibrio

$$
\frac{d P(y)}{d y}=-R(y) g
$$

Esto significa que la presión en cualquier nivel está determinada por el peso total del fluido por encima de ese nivel.

Abordaremos el problema de estabilidad desde el punto de vista de un IVP. Para ello, resolveremos la ecuación diferencial (2.137) en el espacio de frecuencias para la doble transformada de Fourier-Laplace de la componente 
en $y$ de la velocidad perturbada $v_{k s}$. Por simplicidad, y según Ott y Russel [82], elegimos una condición inicial que se corresponde a la función de Green de la ecuación (2.137) que denotamos por

$$
\Gamma(y ; s, k)=\Gamma_{o} \delta\left(y+y_{1}\right)
$$

donde $\delta\left(y+y_{1}\right)$ es la función delta de Dirac centrada en $y=-y_{1}$, siendo $\Gamma_{o}$ una constante.

Las soluciones de la ecuación (2.137) deben satisfacer ciertas condiciones de contorno. Puesto que estamos interesados en modos cuyo comportamiento esté dominado por la discontinuidad de la densidad en la superficie libre de separación de ambos fluidos, unas condiciones de contorno adecuadas serían $v_{k s} \rightarrow 0$ cuando $|y| \rightarrow \infty$. Por otro lado, necesitamos utilizar condiciones de empalme en la superficie de separación de ambos fluidos $\left(y=y_{o}\right)$ donde existe una discontinuidad en la densidad. En relación a estas condiciones de empalme, una condición es que $v_{k s}$ sea continua en la interfase de separación.

Teniendo en cuenta a Chandrasekhar [86] y Sharma et al. [70], utilizaremos una segunda condición de empalme integrando la ecuación (2.137) alrededor de $y=y_{o}$ entre $y_{o}-\varepsilon$ y $y_{o}+\varepsilon$, estableciendo con posterioridad que $\varepsilon \rightarrow 0$. En virtud de la continuidad de $v_{k s}$ a través $y=y_{o}$ y la delimitación de $R$ este proceso límite nos lleva a

$$
\Delta_{o}\left[R \frac{d v_{k s}}{d y}\right]+\frac{g k^{2}}{s^{2}}\left(\Delta_{s} R-\frac{k^{2} T_{s}}{g}\right) v_{k s}=0
$$

donde $\Delta_{o}(f)=f\left(y_{o}+0\right)-f\left(y_{o}-0\right)$ es el salto que una función $f$ experimenta en la interfase $y=y_{o}$. El subíndice $o$ denota el valor de una magnitud continua conocida en la interfase $y=y_{o}$.

\subsection{Relación de dispersión}

Teniendo en cuenta la condición inicial dada por la expresión $(6.3), v_{k s}$ debe ser continua en $y=-y_{1}$. Además, la primera derivada de $v_{k s}\left(y, y_{1} ; s, k\right)$ es discontinua en una cantidad unidad en $y=-y_{1}$, de tal manera que la segunda derivada da lugar a una singularidad tipo delta de Dirac. Así, obtenemos la siguiente condición de salto en $y=-y_{1}$

$$
\frac{d v_{k s}\left(y=-y_{1}^{+}, y_{1} ; s, k\right)}{d y}-\frac{d v_{k s}\left(y=-y_{1}^{-}, y_{1} ; s, k\right)}{d y}=-\frac{\Gamma_{o}}{\rho_{1} s^{2}}
$$


Teniendo en cuenta las condiciones anteriormente mencionadas, la solución general de (2.137) es una combinación lineal de dos soluciones linealmente independientes. Así, $v_{k s}\left(y, y_{1} ; s, k\right)$ resultará ser

$$
v_{k s}\left(y, y_{1} ; s, k\right)=\left\{\begin{array}{cc}
A_{2} e^{k y} & \left(y<y_{1}\right) \\
A_{3} e^{k y}+A_{4} e^{-k y} & \left(-y_{1}<y<0\right) \\
\mathrm{A}_{1} e^{\frac{y(-\alpha-\beta)}{2}} & (y>0)
\end{array}\right.
$$

donde $\alpha$ es dada por la expresión

$$
\alpha=\sqrt{\beta^{2}+4 k^{2}\left(1-\frac{g \beta}{s^{2}}\right)},
$$

siendo $\operatorname{Re}(\alpha)>0$ y las constantes $A_{1}, A_{2}, A_{3}$ y $A_{4}$ son independientes de $y$. Estas constantes están determinadas por las condiciones de frontera y de empalme siendo

$$
\begin{gathered}
A_{1}=\frac{2 \Gamma_{o} e^{-k y_{1}}}{2 k^{4} T_{s}+2 g k^{2}\left(\rho_{1}-\rho_{2}\right)+2 k \rho_{1} s^{2}+(\alpha+\beta) \rho_{2} s^{2}} \\
A_{2}=\frac{\left[2 k^{4} T_{s}+2 g k^{2}\left(\rho_{1}-\rho_{2}\right)+(\alpha+\beta) \rho_{2} s^{2}\right] \Gamma_{o} \sinh \left(k y_{1}\right)}{2 k^{3} \rho_{1}\left[k^{2} T_{s}+g\left(\rho_{1}-\rho_{2}\right)\right] s^{2}+k \rho_{1}\left[2 k \rho_{1}+(\alpha+\beta) \rho_{2}\right] s^{4}} \\
+\frac{2 k \rho_{1} s^{2} \Gamma_{o} \cosh \left(k y_{1}\right)}{2 k^{3} \rho_{1}\left[k^{2} T_{s}+g\left(\rho_{1}-\rho_{2}\right)\right] s^{2}+k \rho_{1}\left[2 k \rho_{1}+(\alpha+\beta) \rho_{2}\right] s^{4}} \\
A_{3}=-\frac{\mathrm{e}^{-k y_{1}} \Gamma_{o}\left[2 k^{4} T_{s}+2 g k^{2}\left(\rho_{1}-\rho_{2}\right)-2 k \rho_{1} s^{2}+(\alpha+\beta) \rho_{2} s^{2}\right]}{2 k \rho_{1} s^{2}\left[2 k^{4} T_{s}+2 g k^{2}\left(\rho_{1}-\rho_{2}\right)+2 k \rho_{1} s^{2}+(\alpha+\beta) \rho_{2} s^{2}\right]} \\
A_{4}=\frac{\Gamma_{o} \mathrm{e}^{-k y_{1}}}{2 k \rho_{1} s^{2}}
\end{gathered}
$$

Por simplicidad, utilizaremos la solución para $y>0$

$$
v_{k s}\left(y, y_{1} ; s, k\right)=\frac{2 \Gamma_{o} e^{-k y_{1}-\frac{y(\alpha+\beta)}{2}}}{\Delta(k, s)}
$$

donde la función de dispersión $\Delta(k, s)$ vendrá dada por

$$
\Delta(k, s)=2 k^{4} T_{s}+2 g k^{2}\left(\rho_{1}-\rho_{2}\right)+2 k \rho_{1} s^{2}+(\alpha+\beta) \rho_{2} s^{2}
$$


Los ceros de la función de dispersión $\Delta(k, s)$ son calculados por la relación de dispersión $\Delta(k, s)=0$, y representan el espectro discreto. Esta expresión puede resolverse para dar lugar a una relación del tipo $s=s(k)$. No es difícil reducir la ecuación $(6.13)$ a una expresión polinómica del tipo

$$
\begin{gathered}
a_{1} s^{4}+a_{2} s^{2}+a_{3}=0 \\
a_{1}=k \rho_{1}^{2}+\beta \rho_{1} \rho_{2}-k \rho_{2}^{2} \\
a_{2}=k\left\{2 k \rho_{1}\left(k^{2} T_{s}+g \rho_{1}\right)+\rho_{2}\left[k^{2} T_{s} \beta+g \rho_{1}(\beta-2 k)\right]\right\} \\
a_{3}=k^{3}\left(k^{2} T_{s}+g \rho_{1}-g \rho_{2}\right)^{2}
\end{gathered}
$$

En consecuencia, $v_{k s}\left(y, y_{1} ; s, k\right)$ tiene dos polos $\pm s_{p}$, i.e. los ceros de la función de dispersión $\Delta(k, s)$. Este resultado es congruente con el resultado obtenido por Obied Allah [67]. Este autor obtuvo la misma relación de dispersión utilizando el análisis en modos normales. Antes de proceder a discutir con detalle los resultados de estos cálculos, consideraremos el comportamiento asintótico de las soluciones de la ecuación (6.14) para pequeños valores de $\beta$. Así, cuando la densidad del fluido de la región $y>0$ se aproxima a un valor constante en el límite de $\beta \rightarrow 0$, la relación de dispersión se convierte en

$$
s^{2}=k g\left[\left(\frac{\rho_{2}-\rho_{1}}{\rho_{2}+\rho_{1}}\right)-\frac{k^{2} T_{s}}{g\left(\rho_{2}+\rho_{1}\right)}\right]
$$

De esta forma, podemos comprobar como la relación de dispersión derivada por Chandrasekhar es recuperada [86]. El espectro continuo aparece como resultado de que $v_{k s}$ es una función multivaluada. Por ello, la expresión 6.12 es la base de nuestra investigación. Siguiendo a Ott y Russel [79], debido a que la función $v_{k s}$ no resulta ser una función par con respecto a $\alpha$, decimos que dicha función tiene puntos de ramificación en $s= \pm s_{b}(\alpha=0)$ sobre el eje real asociados con el espectro continuo donde

$$
s_{b}=\sqrt{\frac{g \beta}{1+(\beta / 2 k)^{2}}}
$$

Si cortamos el plano $s$ a lo largo del intervalo que conecta los puntos de ramificación $-s_{b}$ y $s_{b}, v_{k s}$ se convierte en una función univaluada de $s$. Este 
132 Capítulo 6. El continuum en la RTI con tensión superficial

corte de ramificación $\left[-s_{b},+s_{b}\right]$ se corresponde con el intervalo de existencia del espectro continuo.

La fórmula de inversión es una poderosa técnica para determinar la inversa de la transformada de Laplace. La técnica está basada en los métodos de integración de contorno y requiere que consideremos a nuestro parámetro $s$ como una variable compleja.

A continuación, vamos a invertir $v_{k s}(y, s)$ para obtener $v_{k}(y, t)$ como función del tiempo. Así, la transformada inversa de Laplace de $v_{k s}(y, s)$ vendrá dada por

$$
v_{k}(y, t)=\frac{1}{2 \pi i} \int_{-i \infty+s_{o}}^{+i \infty+s_{o}} v_{k s}(y, s) e^{s t} d s
$$

La expresión (6.20) es conocida como la fórmula compleja de inversión (o de Fourier-Mellin). El contorno de Bromwich $C$ con parte real $\operatorname{Re}(s)=s_{o}$ es un camino apropiado de integración (ver imagen izquierda de la figura 6.2 que exluye las singularidades de $v_{k s}(y, s)$ en la región $R e(s) \geq s_{o}$. Este contorno $C$, recorrido en el sentido indicado, está situado de forma paralela al eje imaginario y la constante $s_{o}$ ha de ser elegida de forma que el camino de integración en el plano complejo $s$ quede a la derecha de todas las singularidades de $v_{k s}$. La evaluación de la integral (6.20) es realizada mediante el método de los residuos y el camino de integración $C_{3}$ es, por tanto, cerrado a la izquierda del semiplano $s$ de tal forma que todas las singularidades de $v_{k s}(y, s)$ queden encerradas (ver imagen derecha de la figura 6.2) por un semicírculo denotado por $C_{R}$. Ahora, deformamos el contorno de integración sin afectar el valor de la integral siempre que dicho contorno no atraviese cualquier singularidad del integrando durante el proceso de deformación. De esta forma, $v_{k s}(y, s)$ se convierte en una función analítica univaluada en la región comprendida entre las líneas $C_{R}+C_{3}, C_{1}, C_{2}$ y $C_{b}$. Según las condiciones de contorno $v_{k s}\left( \pm \infty, y_{1} ; s, k\right)=0$, la raíz cuadrada (6.7) se define de tal manera que $\operatorname{Re}(\alpha)>0$ para $\operatorname{Re}(s)>s_{o}$. Así, esta integral 6.20 en el plano $s$ puede escribirse como

$$
\begin{array}{r}
\int_{C_{R}} v_{k s}(y, s) e^{s t} d s+\int_{C_{3}} v_{k s}(y, s) e^{s t} d s+\int_{C_{1}} v_{k s}(y, s) e^{s t} d s \\
+\int_{C_{2}} v_{k s}(y, s) e^{s t} d s+\int_{C_{b}} v_{k s}(y, s) e^{s t} d s=2 \pi i \sum_{n} \operatorname{Re} s\left[v_{k s}\left(y, s_{n}\right) e^{s_{n} t}\right]
\end{array}
$$

donde Res denota cada valor de los residuos de los polos $s_{n}$ y el sumatorio se 


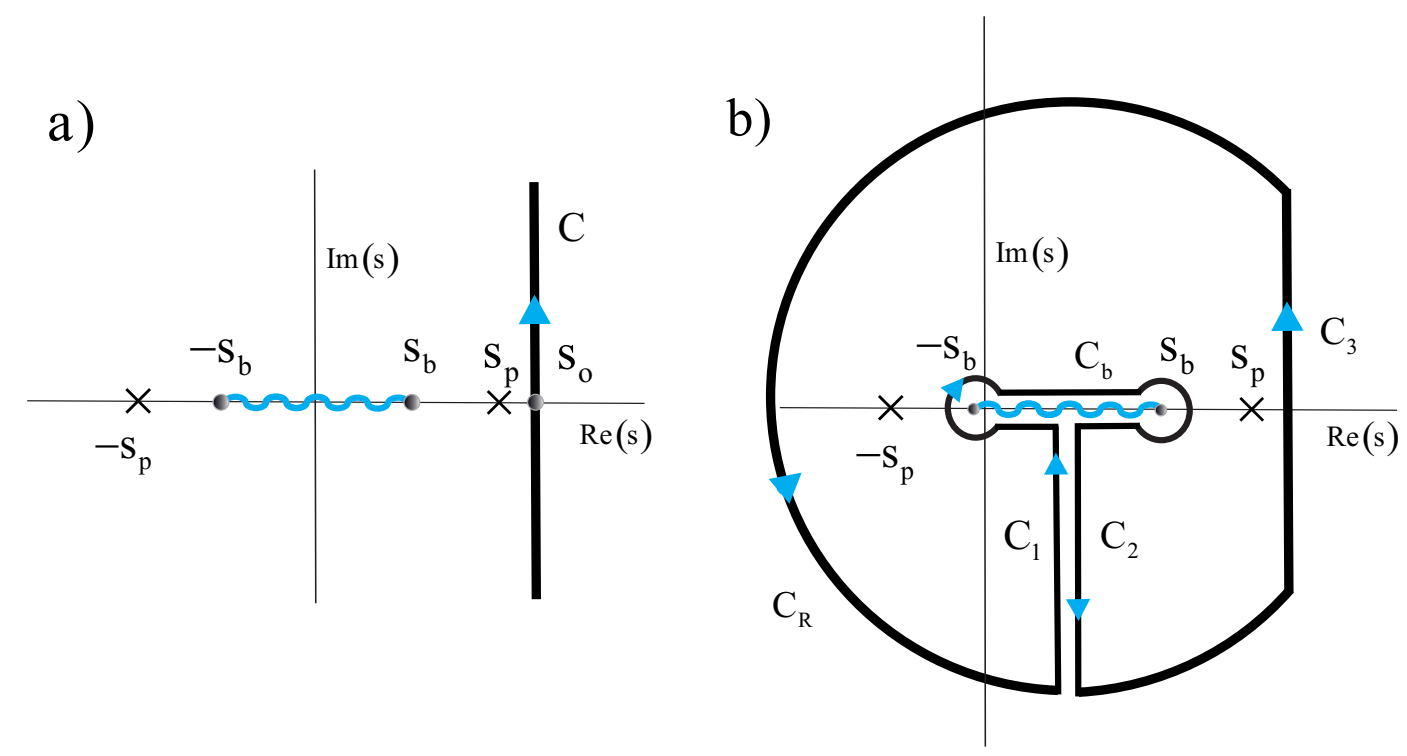

Figura 6.2: a) El camino de integración de Bromwich $C$ con $\operatorname{Re}(s)=s_{o}$. Se aprecian en el eje real los polos $\pm s_{p}$ y los puntos de ramificación $\pm s_{b}$, representando las líneas onduladas el corte de ramificación correspondiente a la hoja de Riemann apropiada. b) Nuevo contorno para la evaluación de la integral 6.20)

extiende a la suma de todos los polos encerrados (dos polos) que representa el espectro discreto.

La integral

$$
\int_{C_{b}} v_{k s}(y, s) e^{s t} d s
$$

puede ser expresada como una combinación lineal de términos del tipo $e^{s t}$ donde $s$ es real y se extiende entre $\pm s_{b}$. Estos valores representan claramente un continuo de autovalores. El corte de ramificación a lo largo del intervalo que conecta $-s_{b}$ y $s_{b}$ se asocia con el espectro continuo inestable de autovalores. Consecuentemente, podemos considerar que 6.22 es la integral asociada al espectro continuo. Puesto que las curvas $C_{R}$ y $C_{3}$ pueden extenderse al infinito y las integrales sobre $C_{1}$ y $C_{2}$ se cancelan mutuamente, los 
134 Capítulo 6. El continuum en la RTI con tensión superficial

primeros cuatro términos en la expresión (6.21) desaparecen, entonces

$$
\begin{aligned}
& v_{k}(y, t)=\frac{1}{2 \pi i} \int_{s_{o}-i \infty}^{s_{o}+i \infty} v_{k s}(y, s) e^{s t} d s=\operatorname{Re} s \sum_{n} v_{k s}\left(y, s_{n}\right) e^{s_{n} t} \\
& -\frac{1}{2 \pi i} \int_{C_{b}} v_{k s}(y, s) e^{s t} d s
\end{aligned}
$$

La condición $\operatorname{Re}(\alpha)>0$ obliga a que la curva $s_{p}^{2}$ que caracteriza a los polos no exista por debajo de la zona inferior de la curva $s_{b}^{2}$ definida por 6.19) y que caracteriza a los puntos de ramificación. Esto da lugar a que las curvas $s_{p}^{2}$ y $s_{b}^{2}$ se corten entre sí para dos puntos de números de onda $k_{c r}^{-}$y $k_{c r}^{+}$ $\left(k_{c r}^{-}<k_{c r}^{+}\right)$que denominaremos números de onda críticos (decimos que para estos números de onda los polos y los puntos de ramificación colapsan). Así, mientras que el espectro continuo existe para todo $k$, el espectro discreto existe solo en el dominio $\left[k_{c r}^{-}, k_{c r}^{+}\right]$.

A continuación, examinaremos el impacto del espectro continuo sobre el espectro discreto.

\subsection{Evolución temporal de la perturbación}

Nuestro objetivo en esta sección es analizar la evolución temporal de una perturbación observando la respuesta transitoria del sistema a dicha perturbación.

\subsubsection{Análisis asintótico para tiempos grandes $(\mathrm{t}>>1)$}

Antes de continuar, sería de interés tratar la naturaleza del crecimiento de la inestabilidad en aquellos casos donde no existe espectro discreto, sino continuo. Podemos estudiar el crecimiento de la respuesta a una perturbación inicial, en primer lugar, para los casos $k<<k_{c r}^{+}$y $k>>k_{c r}^{+}$y, en segundo lugar, para $k=k_{c r}^{-}$y $k=k_{c r}^{+}$. En consecuencia, la única contribución a $v_{k s}(y, s)$ es debida al punto de ramificación en $s=s_{b}$. Para calcular el comportamiento asintótico de $v_{k}(y, t)$, debemos encontrar una expresión aproximada de esta perturbación válida para tiempos grandes.

Para analizar el problema en el espacio de tiempos, tomaremos la transformada inversa de $v_{k s}(y, s)$ integrando a lo largo del contorno $C$. Además, para encontrar el comportamiento asintótico de la integral 6.20), deformamos dicho contorno $C$ hacia la izquierda hasta que encontramos la primera singularidad, la cual es el punto de ramificación en $s_{b}$ (ver Fig. 6.3). A partir de ahora al contorno deformado lo denotaremos como $C^{\prime}$ el cual tiene 


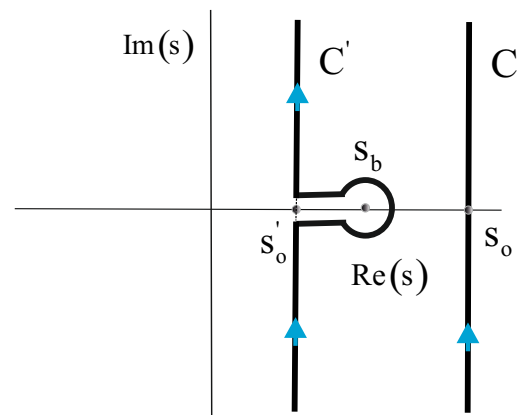

Figura 6.3: Contorno de integración $C^{\prime}$ utilizado para invertir $v_{k s}(y, s)$.

dos tramos verticales con parte real $s_{o}^{\prime}$. Nótese que el valor de $s_{o}^{\prime}$ es mayor que los de las partes reales de todas las singularidades de $v_{k s}$, excepto el correspondiente en $s=s_{b}$.

Para obtener la solución temporal asintótica de $v_{k}(y, t)$, tenemos que exigir que $t$ sea lo suficientemente grande para que la integral a lo largo de $C^{\prime}$ en el tramo $\operatorname{Re}(s)=s_{o}^{\prime}$ pueda ser despreciada cuando la comparamos con la integral obtenida en las proximidades de $s_{b}$. Puesto que tenemos que tomar que el valor de $s_{o}^{\prime}$ sea arbitrariamente cercano a cero, pero ligeramente por encima de este valor, el error que cometeremos al despreciar la integral a lo largo del tramo $\operatorname{Re}(s)=s_{o}^{\prime}$, lejos de $s_{b}$, decrece como $\exp \left(-s_{b} t\right)$ [82]. Por tanto, la solución asintótica puede expresarse como

$$
v_{k}(y, t)=\frac{1}{2 \pi i} \int_{C^{\prime}} v_{k s}(y, s) e^{s t} d s
$$

Usando las expresiones (6.7), 6.12) y 6.19), reemplazamos $s-s_{b}$ por $r e^{i \theta}$ en el integrando de 6.24. En la vecindad del punto de ramificación, reemplazamos $s$ por $s_{b}$ en la expresión 6.24 y obtenemos para $y>0$

$v_{k}=\frac{\Gamma_{o} e^{-k y_{1}-\beta y / 2}}{\pi i} e^{s_{b} t} \int_{C^{\prime}} \frac{\exp \left(-y r^{1 / 2} e^{i \theta / 2} \sqrt{\beta^{2}+4 k^{2}} / \sqrt{2 s_{p}}\right) e^{i \theta} \exp \left(t r e^{i \theta}\right)}{\Delta(r, \theta)} d r$

donde podemos expresar $\Delta(r, \theta)$ como

$$
\begin{aligned}
& \Delta(r, \theta)=2 k^{3} T_{s}+2 g k\left(\rho_{1}-\rho_{2}\right)+2 \rho_{1} s_{b}^{2}+\beta \rho_{2} s_{b}^{2} / k \\
& +\rho_{2} s_{b} r^{1 / 2} e^{i \theta / 2} \sqrt{2 s_{b}} \sqrt{\beta^{2}+4 k^{2}} / k
\end{aligned}
$$




\section{Capítulo 6. El continuum en la RTI con tensión superficial}

Puesto que $t$ es grande $(t>>1)$, la integral de la expresión 6.25 a lo largo del tramo $R e(s)=s_{o}^{\prime}$ es exponencialmente pequeña y la podemos aproximar por

$$
v_{k}=\int_{\infty}^{0} \frac{D e^{i E r^{1 / 2}} e^{-r t}}{B-A r^{1 / 2} i}(-d r)+\int_{0}^{\infty} \frac{D e^{-i E r^{1 / 2}} e^{-r t}}{B+A r^{1 / 2} i}(-d r)
$$

donde las constantes son

$$
\begin{gathered}
A=\rho_{2} s_{b} \sqrt{2 s_{b}} \sqrt{\beta^{2}+4 k^{2}} / k \\
B=2 k^{3} T_{s}+2 g k\left(\rho_{1}-\rho_{2}\right)+2 \rho_{1} s_{b}^{2}+\beta \rho_{2} s_{b}^{2} / k \\
D=\frac{\Gamma_{o} e^{-k y_{1}-\beta y / 2+s_{b} t}}{i \pi k} \\
E=-y \sqrt{\beta^{2}+4 k^{2}} / \sqrt{2 s_{b}}
\end{gathered}
$$

Tomando $r=\rho^{2}$ y $d r=2 \rho d \rho$, podemos escribir 6.27 como

$$
v_{k}=\int_{\infty}^{0} \frac{D e^{E \rho i} e^{-\rho^{2} t}}{B-A \rho i}(-2 \rho d \rho)+\int_{0}^{\infty} \frac{D e^{-E \rho i} e^{-\rho^{2} t}}{B+A \rho i}(-2 \rho d \rho)
$$

introduciendo una nueva variable $x=\rho E$, la expresión anterior la podemos escribir como

$$
v_{k}=\int_{0}^{\infty} \frac{4 D i e^{-(x / E)^{2} t}}{B^{2}+A^{2}(x / E)^{2}}\left[B \sin (x)+\frac{A x}{E} \cos (x)\right] \frac{x}{E^{2}} d x
$$

donde usando las expresiones 6.19, 6.28 y 6.31 podemos definir

$$
\frac{A^{2}}{E^{2}}=\left(\frac{8 \rho_{2} g \beta k / y}{4 k^{2}+\beta^{2}}\right)^{2}
$$


5.1.1 Casos de $k<<k_{c r}^{+} \mathbf{y} k>>k_{c r}^{+}$

En este apartado, vamos a analizar los límites $k<<k_{c r}^{+}$y $k>>k_{c r}^{+}$. Teniendo en cuenta (6.34), en estos casos es fácil mostrar que $B^{2}+(A x / E)^{2} \approx$ $B^{2}$, debido al hecho de que $(A / E)^{2} \rightarrow 0$ cuando $k \rightarrow 0$ y $k \rightarrow \infty$. En este caso la expresión 6.33 se convierte en

$$
v_{k}=\int_{0}^{\infty} \frac{4 D i e^{-(x / E)^{2} t}}{E^{2} B} x \sin (x) d x
$$

Puesto que estamos buscando el comportamiento de $v_{k}(y, t)$ para valores grandes de $t$, la expresión 6.35 puede simplificarse haciendo un desarrollo de Taylor en $x=0$ de la función seno, quedando dicha expresión 6.35 como

$$
v_{k}=\int_{0}^{\infty} \frac{4 D i}{E^{2} B} e^{-(x / E)^{2} t} x^{2} d x
$$

Esta integral definida puede ser fácilmente calculada

$$
v_{k}=\frac{i D \sqrt{\pi}}{B E^{2}}\left(\frac{t}{E^{2}}\right)^{-3 / 2} ; \quad \operatorname{Re}\left(\frac{t}{E^{2}}\right)>0
$$

Si combinamos las expresiones 6.30) y (6.37), obtenemos

$$
v_{k}=\frac{\Gamma_{o} e^{-k y_{1}-\beta y / 2+s_{b} t}}{\pi k} \frac{\sqrt{\pi}}{B E^{2}}\left(\frac{t}{E^{2}}\right)^{-3 / 2}
$$

Concluimos que el comportamiento asintótico de la perturbación para $k<<k_{c r}^{-}$y $k>>k_{c r}^{+}$no es puramente exponencial, para $t>>1$, creciendo en el tiempo como

$$
v_{k}(y, t) \approx t^{-3 / 2} e^{s_{b} t}
$$

\subsubsection{Casos $k=k_{c r}^{-} \mathbf{y} k=k_{c r}^{+}$}

Finalmente, consideraremos el comportamiento asintótico en los límites $k=k_{c r}^{-}$y $k=k_{c r}^{+}$. Aquí, debido al hecho de que en estos números de onda críticos, como se ha establecido anteriormente, los polos y los puntos de ramificación colapsan $\left(s_{p}=s_{b}\right)$, la relación de dispersión se transforma en

$$
\Delta(k, s)=\alpha \rho_{2} s^{2}
$$


138 Capítulo 6. El continuum en la RTI con tensión superficial

Por tanto, la expresión 6.32 puede ser expresada como

$$
v_{k}=\int_{\infty}^{0} \frac{D e^{E \rho i} e^{-\rho^{2} t}}{-A \rho i}(-2 \rho d \rho)+\int_{0}^{\infty} \frac{D e^{-E \rho i} e^{-\rho^{2} t}}{A \rho i}(-2 \rho d \rho)
$$

Realizando el siguiente cambio de variable $x=\rho E$, la expresión 6.41 se transforma

$$
v_{k}=\int_{0}^{\infty} \frac{4 i D e^{-(x / E)^{2} t}}{A E} \cos (x) d x
$$

Aproximando $\cos (x)$ a la unidad en $x=0,6.42$ se convierte

$$
v_{k}=\int_{0}^{\infty} \frac{4 i D e^{-(x / E)^{2} t}}{A E} d x
$$

Después de evaluar la última integral, obtenemos

$$
v_{k}=\frac{4 i D \sqrt{\pi}}{A} t^{-1 / 2}
$$

Entonces, combinando las expresiones 6.44) y (6.30) podemos escribir

$$
v_{k}=\frac{4 \Gamma_{o} \sqrt{\pi}}{\pi k A} e^{-k y_{1}-\beta y / 2+s_{b} t} t^{-1 / 2}
$$

Por lo tanto, la evolución temporal de la perturbación para $t>>1$, $k=k_{c r}^{-}$y $k=k_{c r}^{+}$vendrá dada por

$$
v_{k}(y, t) \approx t^{-1 / 2} e^{s_{b} t}
$$

\subsection{Resultados numéricos}

\subsubsection{Relación de dispersión}

Es útil convertir la expresión (6.12) de $v_{k}$ para $y>0$ a una forma adimensional. Así, la forma adimensional del cuadrado de la tasa de crecimiento, el número de onda, el parámetro de tensión superficial y la coordenada y serán, respectivamente

$$
\bar{s}^{2}=\frac{s^{2}}{g \beta}, \bar{k}=\frac{k}{\beta}, \tau=\frac{\beta^{2} T_{s}}{g \rho_{2}}, \bar{y}=k y,
$$


Asimismo, la forma adimensional del parámetro $\alpha$ y $\Gamma_{o}$ son, respectivamente

$$
\bar{\alpha}=\frac{\alpha}{k}=\sqrt{\frac{1}{\bar{k}^{2}}+4\left(1-\frac{1}{\bar{s}^{2}}\right)}, \bar{\Gamma}_{o}=\frac{\Gamma_{o}}{g \beta \rho_{2}}
$$

Por otro lado, definimos el número de Atwood como

$$
A_{\rho}=\frac{r-1}{r+1}
$$

donde $r=\frac{\rho_{2}}{\rho_{1}}$ representa el salto de densidad. Por conveniencia, escribiremos la función normalizada $v_{k s}(\bar{y}, \bar{s})$ para $\bar{y}>0$ como

$$
v_{k s}(\bar{y}, \bar{s})=\frac{2 \bar{\Gamma}_{o} \exp \left[-\bar{y}_{1}-\bar{y}\left(\bar{\alpha}+\bar{k}^{-1}\right) / 2\right]}{2 \tau \bar{k}^{3}+2 \bar{k}(1 / r-1)+2 \bar{s}^{2} / r+\bar{s}^{2}\left(\bar{\alpha}+\bar{k}^{-1}\right)}
$$

donde el denominador de 6.50 es la relación de dispersión adimensional $\Delta(\bar{k}, \bar{s})$

$$
\Delta(\bar{k}, \bar{s})=2 \tau \bar{k}^{3}+2 \bar{k}(1 / r-1)+2 \bar{s}^{2} / r+\bar{s}^{2}\left(\bar{\alpha}+\bar{k}^{-1}\right)
$$

A continuación, con objeto de simplificar la notación y sin pérdida de generalidad, prescindiremos del símbolo de barra para simbolizar magnitudes adimensionales.

En la figura 6.4 se muestra el cuadrado de la tasa de crecimiento adimensional de la inestabilidad $s^{2}$ frente al número de onda adimensional $k$, obtenida cuando la expresión (6.51) se hace cero $(\Delta(k, s)=0)$ para $A_{\rho}=0.4$ y $A_{\rho}=0.7$, siendo $\tau=0.01$ y $\tau=0.02$. La frontera de la región del plano $\left(k, s^{2}\right)$ que excluye a los dos modos superficiales, ver expresión 6.19, se muestra con una línea discontinua (representa la tasa de crecimiento adimensional al cuadrado de los puntos de ramificación que simbolizaremos por $s_{b}^{2}$ ) en cada gráfica. Por otro lado, las líneas continuas representan el cuadrado de la tasa de crecimiento adimensional de la inestabilidad (espectro discreto) $s_{p}^{2}$. Como podemos apreciar, en cada rama, hay dos puntos terminales con unos números de onda críticos, $k_{c r}^{-}$y $k_{c r}^{+}$, respectivamente, que limitan el dominio de existencia de la relación de dispersión. Como podemos observar, para $k>k_{c r}^{+}$o $k<k_{c r}^{-}$el valor de $s_{p}^{2}$ (polos) se mueve hacia $s_{b}^{2}$ (puntos de ramificación).

Debido a la presencia de la tensión superficial, los modos RTI superficiales (espectro discreto) tienen un máximo y llegan a transformase en modos 
RTI internos en $k_{c r}^{+}$. Esto es posible cuando ponemos de manifiesto la existencia del espectro continuo que no fue tenido en cuenta por Obied [67] en su análisis en modos normales. Así, para $k=k_{c r}^{ \pm}$, entonces $s_{p}^{2}=s_{b}^{2}$ y los polos se desplazan hacia otra hoja de $\alpha$ (los modos superficiales desaparecen). En ausencia del espectro continuo, el dominio del espectro discreto podría ser obtenido igualando la expresión (6.51) a cero. Sin embargo, la intersección del espectro discreto con el continuo en $k_{c r}^{+}$es consecuencia de un efecto combinado de la tensión superficial y la existencia de modos RTI internos creados por un gradiente de densidad, de sentido contrario al campo gravitatorio, en la región de crecimiento exponencial de la densidad.

También podemos observar en la figura 6.4 que para un número de Atwood fijo $A_{\rho}$, cuando $\tau$ aumenta, el dominio $\left[k_{c r}^{-}, k_{c r}^{+}\right]$donde los modos discretos existen se reduce. Por otro lado, cuando incrementamos el valor de $A_{\rho}$ para un valor fijo del parámetro de tensión superficial $\tau$, el dominio de existencia del espectro discreto aumentará. Además, también se muestra cómo los números de onda críticos $k_{c r}^{-} \mathrm{y} k_{c r}^{+}$dependen del salto de densidad, poniéndose de manifiesto la sensibilidad del espectro discreto a los cambios en el número de Atwood $A_{\rho}$.

A nivel secundario, vamos a estimar los valores numéricos para $k_{c r}$, que podemos obtener fácilmente igualando (6.51) a cero. Puesto que $k_{c r}$ depende de $A_{\rho}$ y $\tau$, estudiaremos esta dependencia con $A_{\rho}$ para un parámetro $\tau$ fijo.

Así, en la Fig. 6.5 hemos representado el número de onda crítico $k_{c r}$ en función del número de Atwood $A_{\rho} \mathrm{y}$ el parámetro de tensión superficial $\tau=0.02$. Cada gráfica tiene dos ramas: la rama inferior y la superior para $k_{c r}^{-}$y $k_{c r}^{+}$, respectivamente. También podemos observar en la Fig 6.5 que cuando $A_{\rho}$ disminuye, para un valor fijo $\tau$, el rango en el que el modo superficial existe también decrece. Merece la pena mencionar que para un valor fijo del parámetro $\tau$, cuando el valor de $A_{\rho}$ disminuye, los dos puntos terminales alcanzan los dos valores límites terminales $k_{c r}^{-*}$ y $k_{c r}^{+*}$ sobre cada rama, respectivamente, para un número de Atwood también terminal $A_{\rho}^{*}$.

Estos hechos evidencian que la condición necesaria y suficiente para que exista el espectro discreto, para un valor fijo del parámetro de tensión superficial, es que $A_{\rho}>A_{\rho}^{*}$. Así, por ejemplo, para $\tau=0.01$ los números de onda límites terminales son $k_{c r}^{-*} \approx 3.5395$ y $k_{c r}^{+*} \approx 3.5423$, siendo $A_{\rho}^{*} \approx 0.208942$ el número de Atwood terminal.

Teniendo en cuenta el análisis anterior, el cuadrado de la tasa de crecimiento adimensional frente al número de onda adimensional es representado para $\tau=0.01 \mathrm{y}$ dos números de Atwood diferentes $\left(A_{\rho}=0.22 \mathrm{y}\right.$ $\left.A_{\rho}=0.2089422\right)$ próximos a $A_{\rho}^{*}$, ver Fig. 6.6.

Habría que hacer notar que, aunque el problema analizado aquí es un ca- 

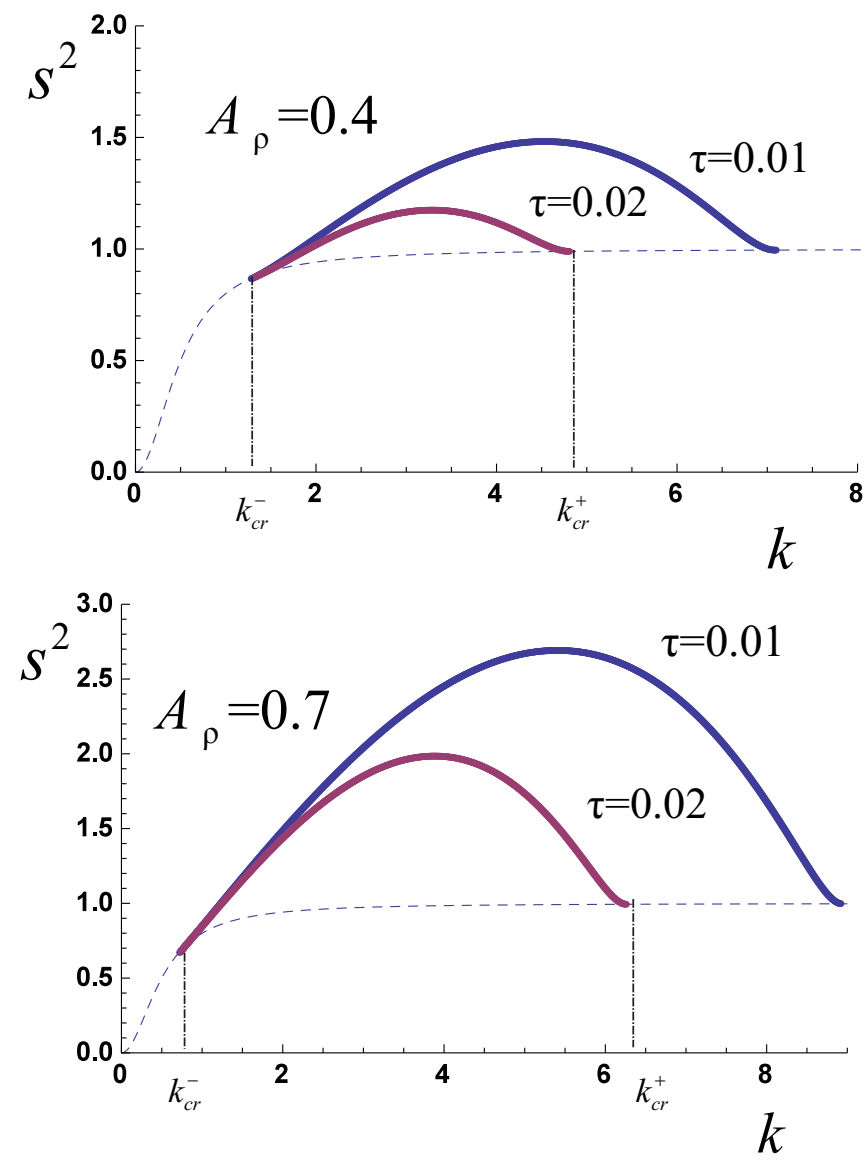

Figura 6.4: Dependencia del cuadrado de la tasa de crecimiento adimensional de la inestabilidad con el número de onda. Los resultados se han obtenido para $\tau=0.01$ y $\tau=0.02$ y diferentes números de Atwood $A_{\rho}=0.4$ (gráfica superior) y $A_{\rho}=0.7$ (gráfica inferior). Las líneas continuas corresponden al espectro discreto. Las líneas discontinuas representan los puntos de ramificación. Por simplicidad, se muestran los números de onda críticos $k_{c r}^{-}$y $k_{c r}^{+}$ para $\tau=0.02$. 

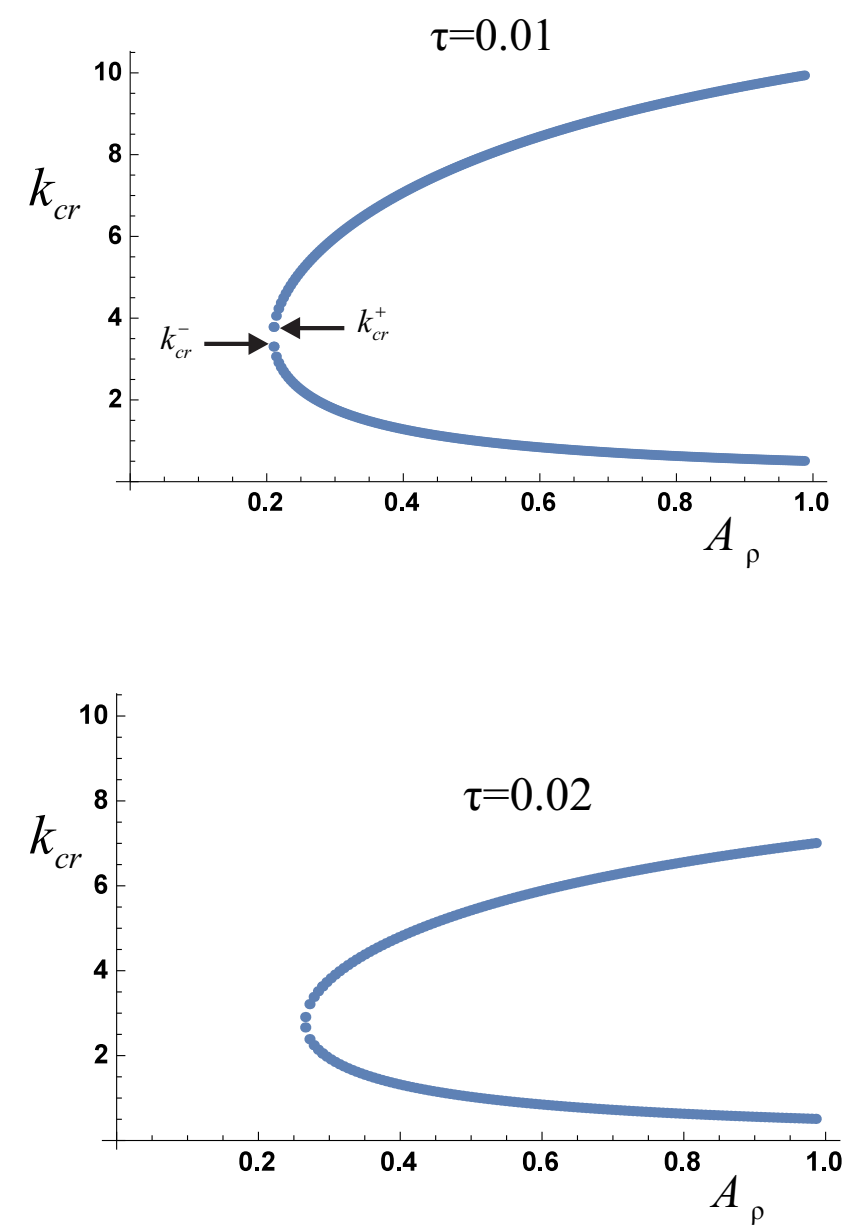

Figura 6.5: Número de onda crítico adimensional $k_{c r}$ en función del número de Atwood $A_{\rho}$ para diferentes valores del parámetro $\tau$. La rama inferior y la superior corresponden a $k_{c r}^{-}$y $k_{c r}^{+}$respectivamente. 

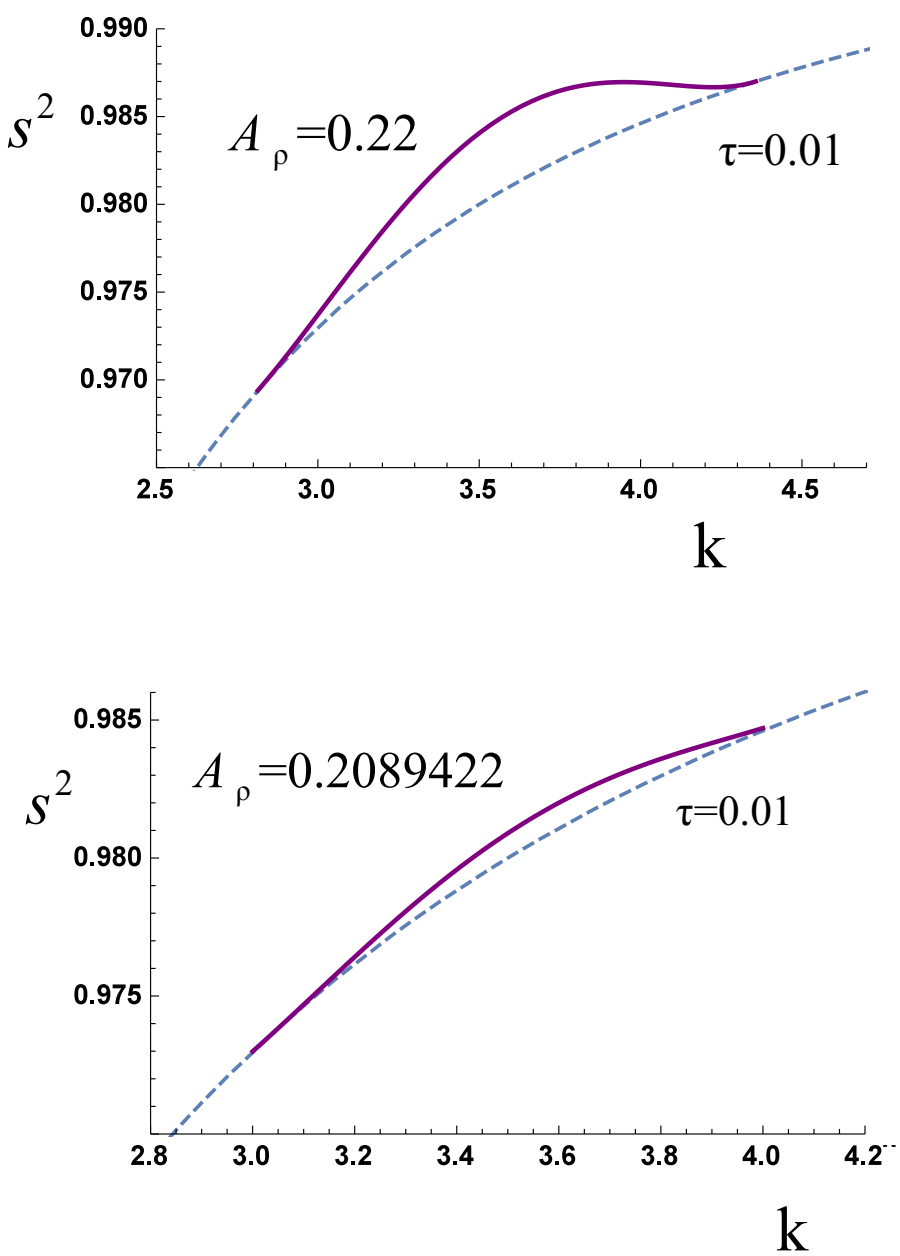

Figura 6.6: Dependencia del cuadrado de la tasa de crecimiento adimensional frente al número de onda adimensional para $\tau=0.01$ y diferentes valores del número de Atwood $A_{\rho}=0.22$ (gráfica superior) y $A_{\rho}=0.2089422$ (gráfica inferior). Las líneas continuas se corresponden con nuestros cálculos numéricos (modos discretos). Las líneas discontinuas representan los puntos de ramificación. 
144 Capítulo 6. El continuum en la RTI con tensión superficial

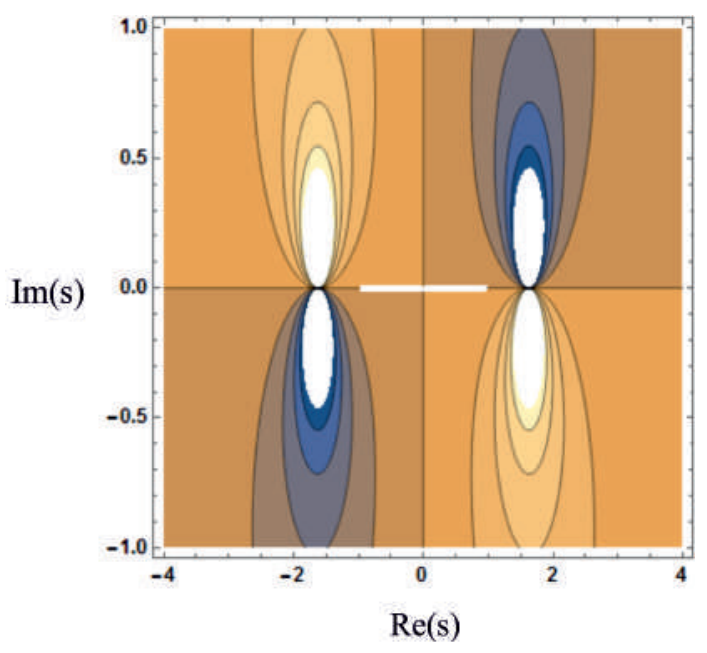

Figura 6.7: Mapa de contorno de la parte imaginaria normalizada de $v_{k} s\left(y, y_{1} ; s, k\right)$ frente a $s$ para $A_{\rho}=0.7, k=6, y_{1}=0.5, y=0, \tau=0.01 \mathrm{y}$ $\Gamma_{o}=1$. Se muestra en una franja blanca el corte de ramificación en el eje real entre $\operatorname{Re}(s)=-1$ y $\operatorname{Re}(s)=1$. Cada pareja de lóbulos representa un polo.

so especial de los considerados por Obied Allah [67], este autor no encontró evidencia de la existencia del espectro continuo. Por otro lado, nuestros resultados parecen refutar la creencia común de que la existencia de la tensión superficial hace posible que la tasa de crecimiento del modo RTI superficial sea cero para un número de onda de corte $k_{c}$ finito. Este resultado no es sorprendente, en el caso analizado, porque el número de onda de corte (entendido como aquel en el que la tasa de crecimiento de la inestabilidad es cero) no existe en esta situación en la que hay un espectro continuo de modos inestables. Además, Obied Allah [67] no hizo ningún comentario explícito sobre la inexistencia de este número de onda de corte.

A continuación, ilustraremos cómo la función normalizada $v_{k s}\left(y, y_{1} ; s, k\right)$ se comporta en función de las partes real e imaginaria de la tasa de crecimiento adimensionalizada de la inestabilidad. Para estudiar gráficamente la existencia del espectro continuo, representaremos un mapa de contorno de la parte imaginaria de $v_{k s}\left(y, y_{1} ; s, k\right)$ para $A_{\rho}=0.7, \tau=0.01, k=6, y_{1}=0.5$, $y=0$ y $\Gamma_{o}=1$, ver Fig. 6.7.

Este hecho ilustra claramente el espectro continuo como un corte de ramificación que se extiende a lo largo del eje real desde $\operatorname{Re}(s)=1$ y $\operatorname{Re}(s)=-1$. Por otro lado, los polos de la función $v_{k s}\left(y, y_{1} ; s, k\right)$ se pueden apreciar como 


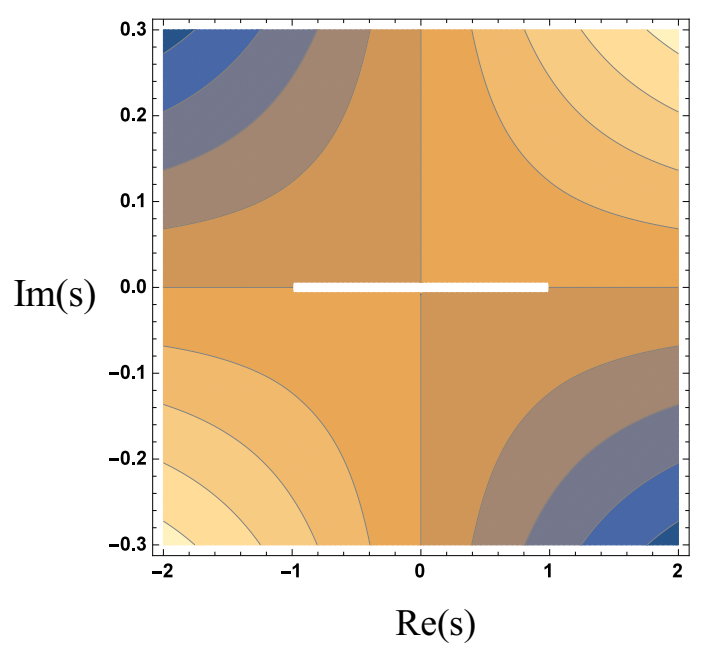

Figura 6.8: Mapa de contorno de la parte imaginaria de $v_{k s}\left(y, y_{1} ; s, k\right)$ frente a $s$ para $A_{\rho}=0.7, k=20, y_{1}=0.5, y=0, \tau=0.01$ y $\Gamma_{o}=1$.

la existencia de parejas de lóbulos en $\operatorname{Re}(s)= \pm 1.626$. Ahora representaremos el mapa de contorno de la parte imaginaria de $v_{k s}\left(y, y_{1} ; s, k\right)$ frente a $s$ para $A_{\rho}=0.7, \tau=0.01, k=20, y_{1}=0.5, \Gamma_{o}=1$ en $y=0$, (ver Fig. 6.8. . En este caso $k_{c r}^{+} \approx 8.9$, puesto $k>k_{c r}^{+}$, no hay espectro discreto y solamente existe un corte de ramificación entre $\operatorname{Re}(s)=1$ y $R e(s)=-1$ que es representativo de la existencia única del espectro continuo.

\subsubsection{Evolución temporal de la perturbación}

Necesitamos realizar un análisis numérico con objeto de estudiar una respuesta más precisa del sistema en todo el rango de tiempos. Por tanto, deberemos calcular la integral de Bromwich

$$
v_{k}(y, t)=\frac{1}{2 \pi i} \int_{C^{\prime}} v_{k s}(y, s) e^{s t} d s
$$

Ahora, por conveniencia, utilizaremos la expresión de $v_{k s}(y, s)$ para $y>0$

$$
v_{k s}(y, s)=\frac{2 \Gamma_{o} \exp \left(-y_{1}-y \varepsilon_{o} / 2\right)}{2 \tau k^{3}+2 k(1 / r-1)+2 s^{2} / r+s^{2} \varepsilon_{o}}
$$

siendo $\varepsilon_{o}$

$$
\varepsilon_{o}=\sqrt{\left(k^{-2}+4\right)\left(s^{2}-s_{b}^{2}\right) / s^{2}}+k^{-1}
$$




\section{Capítulo 6. El continuum en la RTI con tensión superficial}

donde $s_{b}^{2}$ es el cuadrado de la tasa de crecimiento adimensional del punto de ramificación

$$
s_{b}^{2}=\frac{4 k^{2}}{1+4 k^{2}}
$$

\subsubsection{Caso $k_{c r}^{-}<k<k_{c r}^{+}$}

Con objeto de obtener $v_{k}(y, t)$, calculamos la integral 6.52 invirtiendo la transformada de Lapace por el método de inversión de Fourier-Euler (FE). Ahora, vamos a analizar la evolución de la perturbación en diferentes casos.

En la Fig. 6.9 hemos representado $v_{k}$, según las expresiones 6.52 y 6.53), como función del tiempo en $y=7$ para $A_{\rho}=0.7, \tau=0.01, k=6$, $\Gamma_{o}=1$ y $y_{1}=10^{-4}$ donde la contribución del espectro discreto es dominante (ver Fig. 6.4). Para $t>>1$, podemos aproximar la expresión 6.52 por la contribución del espectro discreto y ajustar la señal para grandes valores del tiempo como una función del tipo $v_{k}^{*}(y=7, t)=a e^{s_{p} t}$. La constante $a$ está determinada mediante un ajuste por mínimos cuadrados y resultará ser $a=5.4755 \cdot 10^{-4}$ cuando $s_{p}=1.6255$. Así, obtendremos la siguiente curva ajustada la cual dará la contribución del modo discreto para $t>>1$

$$
v_{k}^{*}(y=7, t)=5.4755 \cdot 10^{-4} e^{1.6255 t}(t>>1)
$$

En la Fig. 6.9 podemos observar un ajuste bastante aceptable para $t>>1$ con la expresión dada por la expresión (6.56).

Teniendo en cuenta la expresión (6.56), es fácil obtener $v_{k}^{*}(y=7, t)$ para todo el rango de tiempos

$$
v_{k}^{*}(y=7, t)=5.4755 \cdot 10^{-4}\left(e^{1.6255 t}-e^{-1.6255 t}\right)
$$

Con objeto de estudiar cómo el espectro continuo afecta a la evolución de la perturbación, representamos en la Fig. 6.10 el cociente $v_{k} / v_{k}^{*}$ frente al tiempo normalizado $t$ para $y=10^{-3}, 1,5,10$. Así, comparamos la respuesta numérica completa $v_{k}$ con la evolución de la perturbación debida exclusivamente al modo discreto $v_{k}^{*}$. Como se puede observar en la figura 6.10, la línea horizontal discontinua es la asíntota a la cual $v_{k} / v_{k}^{*}$ tiende cuando $t \rightarrow \infty$, i.e., en este régimen asintótico, $v_{k} \approx v_{k}{ }^{*}$. En primer lugar, para $y=10^{-3}$, encontramos $v_{k}>v_{k}^{*}$ sobre el intervalo $\left(0, t_{c}\right)$ siendo $t_{c}=5.99$. Este resultado indica que la perturbación completa $v_{k}\left(y=10^{-3}, t\right)$ crece más rápidamente que el modo $v_{k}^{*}\left(y=10^{-3}, t\right)$. Sobre este intervalo, los modos del espectro continuo refuerzan el efecto del modo discreto dando lugar a una respuesta amplificada. 


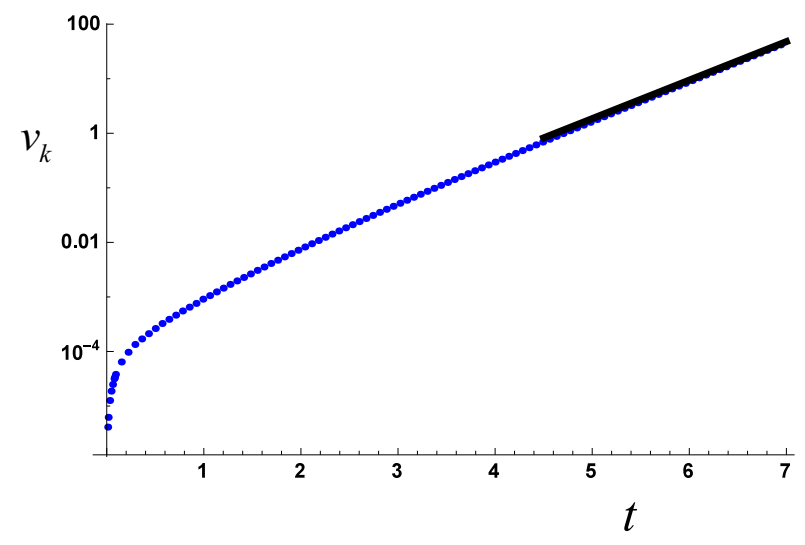

Figura 6.9: Representación de la función normalizada $v_{k}$ como función del tiempo para $A_{\rho}=0.7, \tau=0.01, k=6, \Gamma_{o}=1, y=7$ y $y_{1}=10^{-4}$. La línea sólida representa una función de ajuste de la forma $A e^{s_{p} t}$. La velocidad perturbada la representamos $v_{k}$ sobre una escala logarítmica. En esta gráfica están representadas simultáneamente las contribuciones del espectro continuo y del discreto.

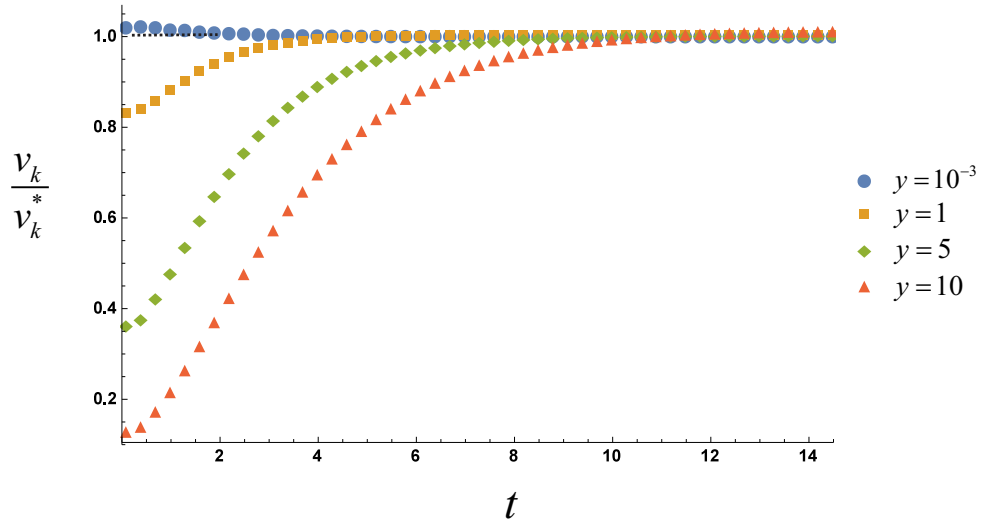

Figura 6.10: Cociente entre la velocidad perturbada total en función de y y la correspondiente al modo discreto para $A_{\rho}=0.7, \tau=0.01, k=6, \Gamma_{o}=1$, $y_{1}=10^{-4}$ y $y=10^{-3}, 1,5,10$. La línea discontinua es la asíntota donde $v_{k} \sim v_{k}^{*}$. 


\section{Capítulo 6. El continuum en la RTI con tensión superficial}

En segundo lugar, consideraremos cómo el espectro continuo afecta a $v_{k}(y, t)$ para grandes valores de $y$. Cuando $y=1,5$ y 10 , debido a la acción del continuo podemos observar que la perturbación global $v_{k}$ crece más lentamente que el modo discreto dado por $v_{k}^{*}$, i.e. la respuesta ha sido reducida por $4 \%, 32 \%$ y $43 \%$ respectivamente (porcentaje medio). Así, según la Fig. 6.10, podemos establecer que cuando $y>1$ el rol del espectro continuo es disminuir la respuesta a la perturbación. Este resultado no es sorprendente, si tenemos en mente que la contribución del espectro continuo está siempre presente.

Estas observaciones ponen de manifiesto que el espectro continuo no debería ser despreciado cuando estudiamos la evolución temporal de la perturbación.

\subsubsection{Casos $k \leq k_{c r}^{-} \mathbf{y} k \geq k_{c r}^{+}$}

Finalmente, para el caso $A_{\rho}=0.7, \tau=0.01, \Gamma_{o}=1, y=10^{-3}$ y $y_{1}=10^{-4}$, sería ilustrativo estudiar el comportamiento asintótico de la evolución de la perturbación cuando solo existe el continuo $\left(k \geq k_{c r}^{+}\right.$y $\left.k \leq k_{c r}^{-}\right)$siendo $k_{c r}^{-}=0.71822$ y $k_{c r}^{+}=8.93074$. Hemos ajustado los datos para $t>>1$ con una función de la forma $a t^{-b} e^{s_{b} t}$ donde $s_{b}$ es el punto de ramificación normalizado. La tabla 6.1 ilustra varios valores de los parámetros de ajuste $a, b$ y $s_{b}$ correspondientes a la expresión $a t^{-b} e^{s_{b} t}$ para diferentes números de onda normalizados.

Habría que mencionar que para $k>k_{c r}^{+}$el exponente $b$ se incrementa con $k$. En primer lugar, consideraremos $k>>k_{c r}^{+}$y elegimos $k=30$. Usando la expresión 6.52, la evolución de la perturbación debido al espectro continuo para $t>>1$ vendrá dada por

$$
v_{k}\left(y=10^{-3}, t\right)=3.883 \cdot 10^{-5} t^{-1.499310} e^{0.999861 t}(t>>1)
$$

Podemos observar que la expresión (6.58) tiene buena coincidencia con la aproximación asintótica dada por la ecuación (6.39) siendo el exponente $b$ en la ecuación 6.58 igual a $b=1.499310 \approx 3 / 2$.

En segundo lugar, para $k<<k_{c r}^{+}$, por ejemplo, $k=0.1$, el ajuste da lugar

$$
v_{k}\left(y=10^{-3}, t\right)=0.2206610 t^{-1.494520} e^{0.196116 t}(t>>1)
$$


En tercer lugar, para $k=k_{c r}^{-}=0.71822$, obtenemos

$$
v_{k}\left(y=10^{-3}, t\right)=0.0571974 t^{-0.503611} e^{0.820709 t}(t>>1)
$$

Y finalmente, para $k=k_{c r}^{+}=8.93074$ podemos escribir

$$
v_{k}\left(y=10^{-3}, t\right)=0.6207830 t^{-0.494106} e^{0.998437 t}(t>>1)
$$

Es importante hacer notar que las expresiones (6.60) y 6.61) están en buen acuerdo con la expresión asintótica analítica (6.46) debido a la dependencia de la velocidad perturbada con $y$, aproximadamente, como $v_{k} \sim t^{-0.5}$ (aquí el exponente $b$ en las expresiones $(6.60)$ y $(6.61)$ es $b \approx 0.5$ ).

Tabla 6.1: Parámetros de ajuste $a, b s_{b}$ en la expresión $v_{k}\left(y=10^{-3}, t\right)=$ $a t^{-b} e^{\left(s_{b} t\right)}$ para diferentes valores de $k$, siendo $\Gamma_{o}=1, A_{\rho}=0.7, \tau=0.01$, $y=10^{-3}$ y $y_{1}=10^{-4}$

\begin{tabular}{llll}
\hline$k$ & $a$ & $b$ & $s_{b}$ \\
\hline 0.10000 & 0.2206610 & 1.494522 & 0.196116 \\
0.20000 & 0.1818642 & 1.479673 & 0.371391 \\
0.30000 & 0.1808763 & 1.434328 & 0.514496 \\
0.40000 & 0.1906886 & 1.352626 & 0.624695 \\
0.50000 & 0.1780879 & 1.201882 & 0.707107 \\
$0.71822\left(k_{c r}^{-}\right)$ & 0.0571974 & 0.505777 & 0.820709 \\
$8.93074\left(k_{c r}^{+}\right)$ & 0.6207830 & 0.494106 & 0.998437 \\
9.00000 & 1.3890063 & 0.907706 & 0.998460 \\
12.0000 & 0.0109028 & 1.424671 & 0.999133 \\
15.0000 & 0.0019315 & 1.463316 & 0.999445 \\
20.0000 & 0.0003093 & 1.463883 & 0.999688 \\
30.0000 & 0.0000388 & 1.476592 & 0.999861 \\
60.0000 & 0.0000029 & 1.498662 & 0.999981 \\
\hline
\end{tabular}

Finalmente, para clarificar los efectos del espectro continuo cuando $t \rightarrow$ 0 , sería recomendable definir el siguiente parámetro $\delta$ como

$$
\delta=1-\frac{v_{k}(y, 0)}{v_{k}^{*}(y, 0)}
$$

el cual es una medida de la contribución del continuo a la respuesta completa del sistema. Así, la dependencia de $\delta$ sobre $y$ para $\tau=10^{-4}, \Gamma_{o}=1, k=12$, 


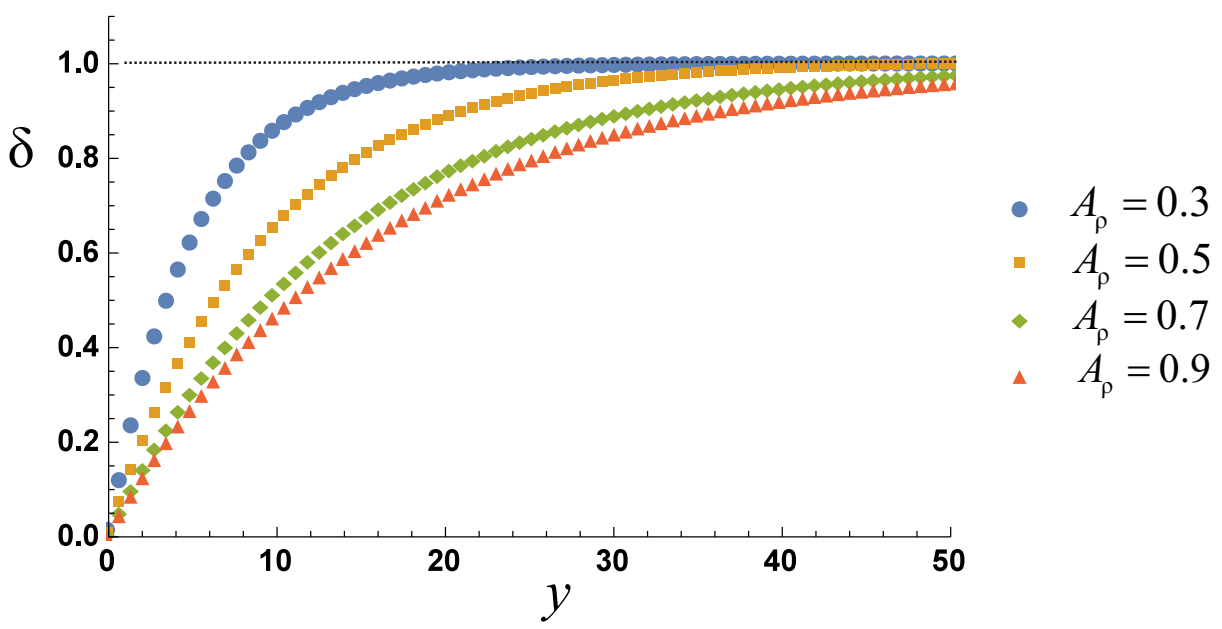

Figura 6.11: Dependencia de $\delta$ en $y$ para $\tau=10^{-3}, \Gamma_{o}=1, k=12, y_{1}=10^{-4}$ y diferentes números de Atwood $A_{\rho}=0.3,0.5,0.7$ y 0.9. La línea discontinua es la asíntota para la cual $\delta \sim 1$.

$y_{1}=10^{-4}$ y diferentes números de Atwood se muestra en la figura 6.11. Como podemos observar en esta figura, para un valor fijo de $A_{\rho}$ cuando $y$ se incrementa, $\delta$ también aumenta, alcanzando la unidad para grandes valores de $y$. Cuando $y$ aumenta, la contribución del continuo domina completamente. Habría que hacer notar que cuando y se incrementa, la saturación de $\delta$ se alcanza más rápidamente cuanto más pequeños son los números de onda. Sin embargo, es destacable que para pequeños valores de $y$ la contribución del continuo es despreciable, siendo este efecto más acusado cuando incrementamos los valores del número de Atwood $A_{\rho}$. Podemos concluir que, para un valor determinado de $y$, el efecto del espectro continuo crece cuando decrece el valor del número de Atwood $A_{\rho}$.

Sería interesante examinar el comportamiento del parámetro $\delta$ cuando $A_{\rho}, \tau$ y $y_{1}$ se mantienen constantes $\left(A_{\rho}=0.7, \Gamma_{o}=1, \tau=10^{-3}\right.$ y $\left.y_{1}=10^{-4}\right)$, donde $\delta$ decrece cuando $y$ se reduce (ver Fig. 6.12). El valor mínimo de $\delta$ siempre se alcanza en $k=16.7156$ el cual corresponde al máximo valor del polo $s_{p}=2.82214$ como era de esperar. 


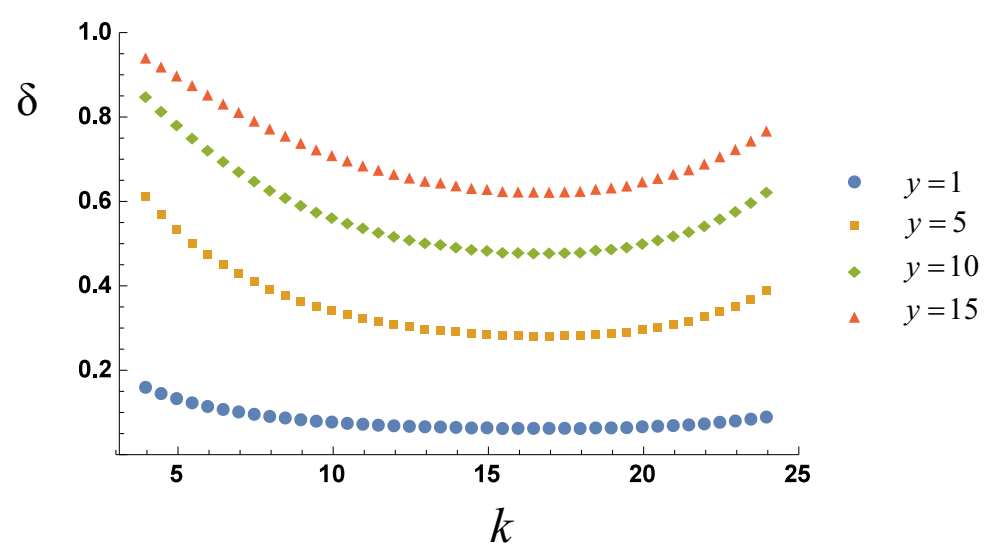

Figura 6.12: Dependencia de $\delta$ con $k$ para $\tau=10^{-3}, y_{1}=10^{-4}, A_{\rho}=0.7$, $\Gamma_{o}=1$ у $y=1,5,10$, y 15 .

\subsection{Resumen y conclusión}

Hemos realizado un análisis lineal de la inestabilidad de Rayleigh-Taylor para dos fluidos incompresibles e inmiscibles con tensión superficial en la superficie libre de separación de los dos fluidos, en un campo gravitatorio uniforme. El problema aquí estudiado se mantiene en el marco del problema analizado por Obied Allah [67, pero en lugar de llevar a cabo un análisis mediante el método de los modos normales, utilizamos la trasformada de Laplace para resolver el correspondiente problema de valor inicial. Cuando elegimos como condiciones iniciales las que se corresponden con la función de Green de la ecuación diferencial de la perturbación, encontramos que esta tiene puntos de ramificación sobre el eje real del plano complejo asociados a la tasa de crecimiento de la inestabilidad. Este enfoque tiene la potencial ventaja de incluir una nueva parte del espectro debida al corte de ramificación en el plano complejo, no tratada por Obied Allah [67], asociado al espectro del continuo de autovalores. En cada rama, debido a la interacción del modo superficial con el espectro continuo que hemos localizado, dos números de onda terminales adimensionales $k_{c r}^{-}$y $k_{c r}^{+}$determinan el intervalo de existencia de la relación de dispersión. Por tanto, puesto que existe un espectro continuo de autovalores, el rol de la tensión superficial es, en este problema, transformar los modos RTI superficiales (espectro discreto) en modos RTI internos (espectro continuo) para un determinado número de onda crítico adimensional $k_{c r}^{+}$. Para el perfil de densidad estudiado, observamos la inexistencia del número de onda de corte (número de onda correspondiente a una 
tasa de crecimiento de la inestabilidad nula). En consecuencia, nuestro descubrimiento parece refutar la creencia común de que, debido a la existencia de la tensión superficial, la tasa de crecimiento de la inestabilidad para el modo superficial RTI se hace cero para un número de onda de corte $k_{c}$, tal y como estudios anteriores parecían evidenciar. Finalmente, debido al espectro continuo, el comportamiento del sistema perturbado es modificado. Así, cuando comparamos la parte dominante del espectro discreto, confirmamos que en la ausencia de espectro discreto, y para valores grandes del tiempo, una perturbación inicial crece temporalmente por el efecto del espectro continuo mediante una función exponencial dependiente del tiempo multiplicada, asimismo, por el tiempo elevado a la potencia de una fracción negativa. 


\section{CAPÍtulo 7 \\ Conclusiones}

\subsection{Resumen de los logros alcanzados}

En primer lugar, hemos analizado numéricamente la inestabilidad de Rayleigh-Taylor viscosa (VRTI) en régimen lineal desde el punto de vista de un análisis modal BiGlobal (formulación 2D) y de un análisis local (formulación 1D). Para ello, hemos planteado un problema de autovalores generalizado con sus correspondientes condiciones de contorno. Con objeto de poder integrar numéricamente las ecuaciones del problema, hemos regularizado la densidad y la viscosidad dinámica en la superficie de separación entre ambas fases fluidas mediante funciones del tipo tangente hiperbólica. Ambos perfiles de densidad y viscosidad tienen un parámetro de control común de especial relevancia que es su longitud característica. Cuando hacemos tender dicha longitud característica a cero, estaríamos modelizando el caso clásico de la VRTI, i.e. una transición abrupta entre ambas fases fluidas. Sin embargo, cuando la mencionada longitud característica es distinta de cero, ya no hablamos de una simple interfase, sino de una interfase difusa o capa de transición, siendo la longitud característica de los perfiles de densidad y viscosidad una medida del espesor de la referida capa. En el caso de una capa de transición entre dos fases fluidas, hemos comprobado la existencia de un espectro discreto de infinitos modos viscosos no estudiados con anterioridad. El cálculo de los autovalores y las autofunciones lo hemos llevado a cabo en las dos formulaciones, obteniendo un buen acuerdo. Para el caso de la transición abrupta, hemos incorporado el efecto de la tensión superficial, ya que hasta la fecha no se habían representado relaciones de dispersión numéricas que incorporasen los efectos del espesor finito de las fases, viscosidad y tensión superficial. En este problema, la ventaja de utilizar la formulación 2D frente a la $1 \mathrm{D}$ es que permite la representación bidimensional de las autofunciones del problema para su posterior análisis.

En segundo lugar, hemos investigado la inestabilidad de Kelvin-Helmholtz lineal en la formulación 2D y en la 1D para un medio de densidad estratificada en un dominio finito. Hemos utilizado como flujo base perfiles de densidad y velocidad del tipo tangente hiperbólica utilizado por Gan et al. 
94 en su simulación numérica, pero a diferencia de estos autores, que estudiaron la inestabilidad para un único número de onda, hemos obtenido una relación de dispersión. Para ello, hemos resuelto numéricamente el problema de autovalores generalizado con sus correspondientes condiciones de contorno, hallando los autovalores y las autofunciones. Se han comparado los autovalores obtenidos en ambas formulaciones obteniéndose un buen acuerdo. Las autofunciones halladas en la formulación 2D han sido representadas $\mathrm{y}$, a partir de las autofunciones de la velocidad perturbada, se ha llevado a cabo una representación de las líneas de corriente con objeto de ubicar en el dominio de interés los vórtices formados. Con respecto al análisis de la relación de dispersión obtenida, es importante mencionar que su comportamiento es diferente al de la relación de dispersión del flujo base de Wang et al. 93] que Gan et al. 994, utilizaron para validar sus resultados. Si la relación de dispersión de Wang et al. [93] crece monótonamente hasta alcanzar un valor asintótico máximo, la relación de dispersión hallada utilizando el flujo base de Gan et al. crece monótonamente hasta alcanzar un máximo para posteriormente decrecer con un aumento del número de onda. Podemos explicar esta diferencia de comportamiento mediante la teoría WIT admitiendo una interacción entre dos interfases difusas de vorticidad en el flujo base de Gan et al.94] y que no ocurre en el de Wang et al. 93]. Por todo ello, concluimos que nuestros resultados podrían ser de gran interés para validar futuras simulaciones numéricas de la KHI del flujo base de Gan et al. 94 para otros números de onda diferentes al utilizado por estos autores. En otro orden de cosas, hemos estudiado la coexistencia simultánea de RTI y KHI en una interfase difusa que se podría entender como el efecto de una velocidad de cortadura sobre la RTI en dicha interfase. Este estudio lo hemos llevado a cabo tanto en una formulación 1D como 2D. Hemos comprobado que la velocidad de cortadura produce una inhibición total del modo la RTI para un número de onda de corte. Esta inhibición podría estar relacionada con la interacción del modo RTI principal con un modo RTI secundario y que sería de interés estudiar en un futuro trabajo.

En tercer lugar, hemos llevado a cabo un estudio de la inestabilidad de vórtices de densidad variable desde un enfoque de análisis BiGlobal. Este estudio se ha llevado a cabo con objeto de complementar problemas ya resueltos. Así, hemos estudiado la inestabilidad centrífuga de un Bqv para un $R e=667$ (estudiada en su momento por Di Pierro y Abid [2] mediante un análisis espacio-temporal), así como la de Rayleigh-Taylor para $R e \rightarrow \infty$, de un vórtice tipo Lamb-Oseen (analizada por Joly et al. [104 mediante un análisis local). En la resolución de los correspondientes EVPs, hemos comparado los autovalores obtenidos con los hallados por los mencionados 
autores, obteniéndose un aceptable acuerdo con Di Pierro y Abid [2] y unas mayores discrepancias con los de Joly et al. [104]. En general, se puede concluir que los autovalores obtenidos en el análisis BiGlobal son inferiores a los obtenidos por los referidos autores. Es decir, los modos que hallamos son más estables de lo esperado. La importancia de nuestro estudio radica en que hemos complementado el trabajo de [104, ya que hemos calculado las autofunciones de la densidad perturbada para determinados autovalores. Asimismo, hemos complementado el trabajo de [2], puesto que se han obtenido las frecuencias de los autovalores, así como las autofunciones de la densidad perturbada.

Por último, hemos analizado la RTI de un fluido no viscoso con tensión superficial en la interfase de separación. Este problema de estabilidad fue estudiado con anterioridad por otro autor Obied Allah [67] reduciéndolo a un EVP. Sin embargo, nuestro estudio se ha realizado desde un enfoque IVP. Hemos obtenido, además del espectro discreto encontrado por este autor, un espectro continuo de autovalores que no es posible encontrar realizando un análisis EVP estándar. A diferencia del espectro discreto que solo existe en un intervalo de números de onda, el continuo existe para cualquier número de onda. Asimismo, hemos encontrado que cuando únicamente se excitan modos del espectro continuo, el crecimiento de la inestabilidad difiere del puramente exponencial.

\subsection{Trabajo futuro}

Como hemos mencionado en el apartado anterior, una línea de investigación en un futuro sería estudiar más a fondo el efecto de una velocidad de cortadura sobre la RTI en perfiles suaves de densidad. Es decir, explicar la inhibición de la RTI por la posible interacción del modo RTI principal con un modo RTI secundario.

Asimismo, otra línea de investigación sería generalizar nuestros cálculos a geometrías más complejas como podría ser la presencia de cuerpos flotantes. De hecho, es una realidad que lo que se pretende hacer en el caso del cálculo de las inestabilidades de un flujo con superficie libre, solución de las ecuaciones de Navier-Stokes, es combinar dos problemas de los cuales por separado se tiene una buena dosis de conocimiento, pero que juntos no se han estudiado con detenimiento. Por una parte tenemos toda la teoría de inestabilidad de flujos bifásicos, entre los cuales se tiene como caso más conocido la inestabilidad de Rayleigh-Taylor. Por otra parte, tenemos casos como el estudio de las inestabilidades producidas en las estelas de cuerpos 
romos como, por ejemplo, un cilindro en el cual se han estudiado los distintos regímenes de estabilidad en función del número de Reynolds y la longitud de onda transversal. La combinación de ambos problemas nos llevaría a un problema donde un cuerpo romo como, por ejemplo, un cilindro estaría sumergido en un fluido bifásico a una profundidad $H$ de la superficie libre y donde se llega a una solución estacionaria para ciertos valores de $H$ y $R e$. Por ello, uno de los futuros trabajos podría basarse en el estudio de la estabilidad de esa solución que, según muestra la teoría, se podría producir por inestabilidades de la estela o por inestabilidades de una superficie libre deformada por la presencia del cilindro. Esta última posibilidad podría ser explorada en un futuro.

Precedentes de este tipo de estudios serían los trabajos realizados por Triantafyllou y Dimas [118, donde se hace una aproximación mediante un flujo base muy simplificado. 


\section{Bibliografía}

[1] K. O. Mikaelian. Rayleigh-Taylor instability in finite-thickness fluids with viscosity and surface tension. Phys. Rev. E, 54(4):3676, 1996.

[2] B. Di Pierro and M. Abid. Spatiotemporal instability of a variabledensity Batchelor vortex. J. Fluid Mech., 703:49-59, 2012.

[3] D. Fabre and L. Jacquin. Viscous instabilities in trailing vortices at large swirl numbers. J. Fluid Mech., 500:239-262, 2004.

[4] I. Delbende, J. M. Chomaz, and P. Huerre. Absolute/convective instabilities in the Batchelor vortex: a numerical study of the linear impulse response. J. Fluid Mech., 355:229-254, 1998.

[5] Lord Rayleigh. On the stability of certain fluid motions. Proc. London Math. Soc., 11:57-70, 1880.

[6] O. Reynolds. An experimental investigation of the circumstances which determine whether the motion of water shall be direct or sinuous, and of the law of resistance in parallel channels. Phil. Trans. Royal Soc. London, 174:935-982, 1883.

[7] J. S. Trefil. Introduction to the Physics of Fluids and Solids. Dover, New-York, 2010.

[8] P. G. Drazin. Introduction to Hydrodynamic Stability. Cambridge University Press, 2002.

[9] H. B. Keller. Numerical solution of bifurcation and nonlinear eigenvalue problems. In Applications of Bifurcation Theory, ed. P Rabinowitz, pp. 359-84. New York: Academic, 1977.

[10] E. Askervik, L. Brandt, D. S. Henningson, J. Hoepffner, O. Marxen, and P. Schlatter. Steady solutions of the Navier-Stokes equations by selective frequency damping. Phys. Fluids, 18:068102, 2006.

[11] P. Schmid and D. Henningson. Stability and Transition in Shear Flows. New York: Academic, 2001.

[12] R. T. Pierrehumbert and S. E. Widnall. The two- and threedimensional instabilities of a spatially periodic shear layer. J. Fluid Mech., 114:59-82, 1982. 
[13] L. E. Eriksson and A. Rizzi. Computer-aided analysis of the convergence to steady state of discrete approximations to the euler equations. J. Comput. Phys., 57:90-128, 1985.

[14] R. T. Pierrehumbert. Universal shortwave instability of twodimensional eddies in an inviscid fluid. Phys. Rev. Lett., 57:2157-59, 1986.

[15] A. Tezuka and K. Suzuki. Three-dimensional global linear stability analysis of fow around a spheroid. AIAAJ, 44:1697-1708, 2006.

[16] V. Theofilis. Global linear instability. Annu. Rev. Fluid Mech., 43(31952), 2011.

[17] G. E. Karniadakis and S. Sherwin. Spectral/hp Element Methods for Computational Fluid Dynamics. Oxford Science Publications, 2005.

[18] F. Longueteau and J. P. Brazier. BiGlobal stability computations on curvilinear meshes. C. R. Méc., 336:828-834, 2008.

[19] E. Piot, G. Casalis, and U. Rist. Stability of the laminar boundary layer flow encountering a row of roughness elements: Biglobal stability approach and DNS. Eur. J. Mech. B Fluids, 27:684-706, 2008.

[20] D. Barkley, H. M. Blackburn, and S. J. Sherwin. Direct optimal growth analysis for timesteppers. Int. J. Numer. Methods Fluids, 57:1435-58, 2008 .

[21] M. H. Carpenter, M. Choudhari, L. Fei, C. L. Streett, and C. L. Chang. Excitation of cross-flow instabilities in a swept wing boundary layer. In Aerosp. Sci. Meet. Exhib., 48th, Orlando, AIAA Pap. 2010-0378, 2010 .

[22] T. Herbet. Parabolized stability equations. Annu. Rev. Fluid Mech., 29:245-83, 1997.

[23] B. Di Pierro, M. Abid, and M. Amielh. Experimental and numerical investigation of a variable density swirling-jet stability. Phys. Fluids, 25:084104, 2013.

[24] G. K. Batchelor. Axial flow in trailing line vortices. J. Fluid Mech., 20:645-658, 1964.

[25] M. Lessen, P. Singh, and F. Paillet. The stability of a trailing line vortex. Part 1. Inviscid theory. J. Fluid Mech., 63:753-763, 1974. 
[26] M. Lessen and F. Paillet. The stability of a trailing line vortex. Part 2. Viscous theory. J. Fluid Mech., 65(4):769-779, 1974.

[27] P. Billant and F. Gallaire. A unified criterion for the centrifugal instabilities of vortices and swirling jets. J. Fluid Mech., 734:5-35, 2013.

[28] R. L. Ash and M. R. Khorrami. Vortex stability. (ed. S. I. Green), chap. 8, pp. 317-372. Kluwer Academic Publishers, 1995.

[29] S. Leibovich and K. Stewartson. A sufficient condition for the instability of columnar vortices. J. Fluid Mech., 126:335-356, 1983.

[30] J. Delery. Aspects of vortex breakdown. Prog. Aerospace. Sci., 30(159), 1994.

[31] K. Sterwartson. The stability of swirling flows at large Reynolds number when subjected to disturbances with large azimuthal wavenumber. Phys. Fluids, 25:1953-1957, 1982.

[32] E. W. Mayer and K. G. Powell. Viscous and inviscid instabilities of a trailing vortex. J. Fluid Mech., 245:91-114, 1992.

[33] M. R. Khorrami. On the viscous modes of instability of a trailing line vortex. J. Fluid Mech., 225:197-212, 1991.

[34] M. R. Khorrami. Behavior of asymmetric unstable modes of a trailing line vortex near the upper neutral curve. Phys. Fluids A, 4:1310-1313, 1992.

[35] S. Le Dizès and D. Fabre. Large-Reynolds-number asymptotic analysis of viscous centre modes in vortices. J. Fluid Mech., 585:153-180, 2007.

[36] C. J. Heaton, J. W. Nichols, and P. J. Schmid. Global linear stability of the non-parallel Batchelor vortex. J. Fluid Mech., 629:139-160, 2009 .

[37] L. M. González, V. Theofilis, and F. Meseguer-Garrido. Applications of high order methods to vortex instability calculations. Spectral and High Order Methods for Partial Differential Equations. Lecture Notes in Computational Science and Engineering, 76:403-410, 2010.

[38] A. Michalke. On the inviscid instability of the hyperbolic-tangent velocity profile. J. Fluid Mech., 19:543-56, 1964. 
[39] A. Michalke. On spatially growing disturbances in an inviscid shear layer. J. Fluid Mech., 23:521-544, 1965.

[40] P. G. Drazin and L. N. Howard. The instability to long waves of unbounded parallel inviscid flow. J. Fluid Mech., 14(2):257-283, 1962.

[41] P. Freymuth. On transition in a separated laminar boundary layer. $J$. Fluid Mech., 25:683-704, 1966.

[42] J. Holmboe. On the behaviour of symmetric waves in stratified shear layers. Geophys. Publ., 24:67-112, 1962.

[43] A. Guha and R. Raj. On the inertial effects of density variation in stratified shear flows. Phys. Fluids, 30:126603, 2018.

[44] J. R. Carpenter, E. W. Tedford, E. Heifetz, and G. A. Lawrence. Instability in stratified shear flow: Review of a physical interpretation based on interacting waves. Appl. Mech. Rev., 64(6):2011, 2011.

[45] Lord Rayleigh. Investigation of the character of the equilibrium of an incompressible heavy fluid of variable density. Proc. London Math Soc, s1-14(1):170-177, 1883.

[46] G. I. Taylor. Instability of liquid surfaces when accelerated in a direction perpendicular to their planes. Proc. R. Soc. London, 192:201, 1950.

[47] D. Lewis. The instability of liquid surfaces when accelerated in a direction perpendicular to their planes. Proc. R. Soc. London, Ser. A, 201:192-196, 1950.

[48] S. Sazonov. Dissipative structures in the F-region of the equatorial ionosphere generated by Rayleigh-Taylor instability. Planet. Space. Sci., 39(12):1667, 1991.

[49] W. Wilcock and J. Whitehead. The Rayleigh-Taylor instability of an embedded layer of low-viscosity fluid. J. Geophys. Res., 96(B7):1219312200, 1991.

[50] J. Arons and S. Lea. Accretion onto magnetized neutron stars - structure and interchange instability of a model magnetosphere. Astrophys. J., 207:914-936, 1976.

[51] K. Anderson and R. Betti. Laser-induced adiabat shaping by relaxation in inertial fusion implosions. Phys. Plasmas, 11(1):5-8, 2004. 
[52] J. Finn. Nonlinear interaction of Rayleigh-Taylor and shear instabilities. Phys. Fluids B, 5(2):415-432, 1993.

[53] W. Amatucci, D. Walker, G. Ganguli, J. Antoniades, D. Duncan, J.H. Bowles, V.V. Gavrishchaka, and M.E. Koepke. Plasma response to strongly sheared flow. Phys Rev Lett., 77(10):1978-1981, 1996.

[54] J. Yang, A. D'Onofrio, S. Kalliadasis, and A. De Wit. RayleighTaylor instability of reaction-diffusion acidity fronts. J. Chem. Phys., 117(20):9395-9408, 2002.

[55] A. Tavakoli, L. Hadzievski, and D. D. Tskhakayab. Rayleigh-Taylor instability of magnetized density transition layer. Phys. Plasmas, 7(1):89-92, 2000.

[56] C. X. Yu, C. Xue, J. Liu, X. Y. Hu, Y. Y. Liu, W. H. Ye, L. F. Wang, J. F. Wu, and Z. F. Fan. Multiple eigenmodes of the Rayleigh-Taylor instability observed for a fluid interface with smoothly varying density. Phys. Rev. E, 97:013102, 2018.

[57] M. Dong, Z. Fan, and C. Yu. Multiple eigenmodes of the RayleighTaylor instability observed for a fluid interface with smoothly varying density. II. Asymptotic solution and its interpretation. Phys. Rev. E, 99:013109, 2019.

[58] S. Chandrasekhar. The character of the equilibrium of an incompressible heavy viscous fluid of variable density. Proc. Camb. Phil. Soc., 51:162-178, 1955.

[59] R. V. Morgan, O. A. Likhachev, and J. W. Jacobs. Rarefaction-driven Rayleigh-Taylor instability. Part 1. Diffuse-interface linear stability measurements and theory. J. Fluid Mech., 791:34-60, 2016.

[60] R. E. Duff, F. H. Harlow, and C. W. Hirt. Effects of stratification, surface tension and rigid planes on Rayleigh-Taylor instability. Phys. Fluids, 5:417-425, 1962.

[61] W. C. Elmore and M. Heald. Physics of waves. Dover, New-York, 1985.

[62] R. Bellman and R. H. Pennington. The character of the equilibrium of an incompressible heavy viscous fluid of variable density. Quart. Appl. Math., 12:151, 1954. 
[63] K. O. Mikaelian. Rayleigh-Taylor and Richtmyer-Meshkov instabilities in multilayer fluids with surface tension. Phys. Rev. A, 42, 1990.

[64] W. H. Reid. The effect of surface tension and viscosity on the stability of two superposed fluids. Proc. Camb. Philos. Soc., 57:415, 1961.

[65] B. J. Daly. Numerical study of the effect of surface tension on interface instability. Phys. Fluids, 12:1340, 1969.

[66] M. Chertkov, I. Kolokolov, and V. Lebedev. Effects of surface tension on immiscible Rayleigh-Taylor turbulence. Phys. Rev. E, 71:055301, 2005.

[67] M. H. Obied Allah. Effects of stratification, surface tension and rigid planes on Rayleigh-Taylor instability. Ind. J. pure appl. Math., 32(3):303, 2001.

[68] D. S. Vaghela and R. K. Chhajlani. Rayleigh-Taylor instability of magnetized partially-ionized superposed fluids with rotation and surface tension in porous medium. Astrophys. Space Sci., 149:301, 1988.

[69] N. F. El-Ansary, G. A. Hoshoudy, A. S. Abd-Elrady, and A. H. A. Ayyad. Effects of surface tension and rotation on the Rayleigh-Taylor instability. Phys. Chem. Chem. Phys., 4:1464, 2002.

[70] P. K. Sharmaa, P. K. Prajapati, and R. K. Chhajlani. Effect of surface tension and rotation on Rayleigh-Taylor instability of two superposed fluids with suspended particles. Acta Physica Polonica A, 118:576, 2010.

[71] X. Ribeyre, V. T. Tikhonchuk, and S. Bouquet. Compressible Rayleigh-Taylor instabilities in supernova remnants. Phys. Fluids, 16(12):4661, 2004.

[72] D. Livescu. Compressibility effects on the Rayleigh-Taylor instability growth between immiscible fluids. Phys. Fluids, 16:118, 2004.

[73] M. A Lafay, B. Le Creurer, and S. Gauthier. Compressibility effects on the Rayleigh-Taylor instability between miscible fluids. EPL, 79:64002, 2007.

[74] K. M. Case. Hydrodynamic stability and the inviscid limit. J. of Fluid Mech., 10:420, 1961. 
[75] K. M. Case. Taylor instability of an inverted atmosphere. Phys. Fluids, 3:366, 1960.

[76] K. M. Case. Stability of an idealized atmosphere. Phys. Fluids, 3:149, 1960.

[77] A. Burger. Instability associated with the continuous spectrum in a baroclinic flow. J. Atmos. Sci., 23, 1966.

[78] Z. Sedlácêk. Electrostatic oscillations in cold inhomogeneous plasma I. Differential equation approach. J. of Plasma Phys., 5:239, 1971.

[79] E. Ott and D. A. Russell. Diffuse-boundary Rayleigh-Taylor instability. Phys. Rev. Lett., 41:1048, 1978.

[80] R. Menikoff, R. C. Mjolsness, D. H. Sharp, C. Zemach, and B. J. Doyle. Initial value problem for Rayleigh-Taylor instability of viscous fluids. The Physics of Fluids, 21(10):1674-1687, 1978.

[81] L. Håkan Gustavsson. Initial-value problem for boundary layer flows. The Physics of Fluids, 22(9):1602-1605, 1979.

[82] D. A. Russell and E. Ott. The linear theory of the Rayleigh-Taylor instability in the equatorial ionosphere. J. Geophys. Res., 84:6573, 1979 .

[83] T. J. Bogdan and P. S. Cally. Waves in magnetized polytropes. Proceedings of the Royal Society A: Mathematical, Physical and Engineering Sciences, pages 943-961, 1997.

[84] H. Von Helmholtz. About discontinuous fluid movements. Mon. Rep. R. Prussian Acad. Sci. Berl., 23:215, 1868.

[85] W. Thomson. Hydrokinetic solutions and observations. Phil. Mag., 42(4):362, 1871.

[86] S. Chandrasekhar. Hydrodynamic and Hydromagnetic Stability. Dover, New-York, 1981.

[87] J. R. D. Francis. Wave motions and the aerodynamic drag on a free oil surface. Phil. Mag. Ser., 7(45):695, 1954.

[88] E. Parker. Dynamics of the interplanetary gas and magnetic fields. Astrophys. J., 128:6640, 1958. 
[89] M. Birkinshaw. Advanced Topics on Astrophysical and Space Plasmas. E. G. Dalpino and A. Peratt and G. M. Tanco and A. Chian editores (Kluwer, Dordrecht, 1997), 1997.

[90] J. Brandt and D. Mendis. Solar System Plasma Physics, Vol. 2. C. F. Kennel et al., North-Holland, Amsterdam, 1979.

[91] B. A. Remington, R. P. Drake, and D. D. Ryutov. Experimental astrophysics with high power lasers and Z pinches. Rev. Mod. Phys., $78: 755,2006$.

[92] S. Pfalzner. An Introduction to Inertial Confinement Fusion (Series in Plasma Physics). Taylor \& Francis Group, 2006.

[93] L. F. Wang, C. Xue, W. H. Ye, and Y. J. Li. Destabilizing effect of density gradient on the Kelvin-Helmholtz instability. Phys. Plasmas, $16: 112104,2009$.

[94] Y. Gan, A. Xu, G. Zhang, and Y. Li. Lattice boltzmann study on Kelvin-Helmholtz instability: Roles of velocity and density gradients. Phys. Rev. E, 83:056704, 2011.

[95] M. Faganello, F. Califano, and F. Pegoraro. Competing mechanisms of plasma transport in inhomogeneous configurations with velocity shear: The solar-wind interaction with Earth's magnetosphere. Phys. Rev. Lett., 100:015001, 2008.

[96] G. A. Hoshoudy and H. Cavus. Kelvin-Helmholtz instability of two finite-thickness fuid layers with continuous density and velocity proiles. J. Astrophys. Astr., 39:39, 2018.

[97] I. Zhelyazkov. On modeling the Kelvin-Helmholtz instability in solar atmosphere. J. Astrophys. Astr., 36(1):233, 2015.

[98] T. V. Zaqarashvili, Z. Vörös, and I. Zhelyazkov. Kelvin-Helmholtz instability of twisted magnetic flux tubes in the solar wind. $A \& A$, 561:A62, 2014.

[99] J. Fontane and L. Joly. The stability of the variable-density KelvinHelmholtz billow. J. Fluid Mech., 612:237-260, 2008.

[100] P. N. Guzdar, P. Satyanarayana, J. D. Huba, and S. L. Ossakow. Influence of velocity shear on the Rayleigh-Taylor instability. Geophys. Res. Lett., 9(5):547-550, 1982. 
[101] L. F. Wang, W. H. Ye, and Y. J. Li. Combined effect of the density and velocity gradients in the combination of Kelvin-Helmholtz and Rayleigh-Taylor instabilities. Phys. Plasmas, 17:042103, 2010.

[102] W. H. Ye, L. F. Wang, C. Xue, Z. F. Fan, and X. T. He. Competitions between Rayleigh-Taylor instability and Kelvin-Helmholtz instability with continuous density and velocity profiles. Phys. Plasmas, 18:022704, 2011.

[103] D. Sipp, D. Fabre, S. Michelin, and L. Jacquin. Stability of a vortex with a heavy core. J. Fluid. Mech., 526:67-76, 2005.

[104] L. Joly, J. Fontane, and P. Chassaing. The Rayleigh-Taylor instability of two-dimensional high-density vortices. J. Fluid Mech., 537:415-431, 2005.

[105] B. Di Pierro and M. Abid. Instabilities of variable-density swirling flows. Phys. Rev. E, 82:046312, 2010.

[106] B. Di Pierro and M. Abid. Rayleigh-Taylor instability in variable density swirling flows. Eur. Phys. J. B., 25(85:69):1-8, 2012.

[107] F. Gallaire and J.M. Chomaz. Mode selection in swirling jet experiments: a linear stability analysis. J. Fluid Mech., 494:223-253, 2003.

[108] Lloyd N. Trefethen. Spectral Methods in MATLAB. SIAM, Philadelphia, 2000.

[109] C. Sánchez del Río. Los principios de la Física en su evolución histórica. Instituto de España, Madrid, 2004.

[110] V. Theofilis. Advances in global linear instability analysis of nonparallel and three-dimensional flows. Prog. Aero. Sci., 39:249-315, 2003.

[111] V. Theofilis, P. W. Duck, and J. Owen. Viscous linear stability analysis of rectangular duct and cavity flows. J. Fluid. Mech., 505:249-286, 2004 .

[112] M. R. Spiegel. Fórmulas y tablas de Matemática aplicada. McGrawHill, 2014.

[113] R. J. Goldstone and P. H. Rutherford. Introduction to Plasma Physics. Taylor \& Francis, New York, 1995. 
[114] G. Arfken. Mathematical Methods for Physicists. Academic Press Limited, London, 1985.

[115] F. C. Hildebrand. Methods of Applied Mathematics. Dover, New-York, 1992.

[116] L. M. González, V. Theofilis V, and R. Gómez-Blanco. Finite-element numerical methods for viscous incompressible biglobal linear instability analysis on unstructured meshes. AIAA Journal, 45(4):840-855, April 2007.

[117] Y. Saad. Variations of Arnoldi's method for computing eigenelements of large unsymmetric matrices. Lin. Algebra. Appl., 34:269-295, 1980.

[118] G. S. Triantafyllou and A. A. Dimas. Interaction of two-dimensional separated flows with a free surface at low froude numbers. Physics of Fluids A: Fluid Dynamics, 1(11):1813-1821, 1989. 